\title{
Analyzing the effects of laquinimod on innate and adaptive immunity in mice with experimental autoimmune encephalomyelitis
}

\author{
Dissertation \\ In partial fulfillment of the requirements for the degree \\ “Doctor rerum naturalium (Dr. rer. nat.)" \\ In the Molecular Medicine Study Program \\ at the Georg-August-University Goettingen
}

Submitted by

Martina Ott

(née Hauck)

from Bad Windsheim, Germany

Goettingen 2014 


\title{
Members of the Thesis Committee
}

\author{
Supervisor (Reviewer)
}

Prof. Dr. Wolfgang Brück

Department of Neuropathology

University Medical Center, Georg-August-University Goettingen

\section{Second Member of the Thesis Committee (Reviewer)}

Prof. Dr. Holger Reichardt

Department of Cellular and Molecular Immunology

University Medical Center, Georg-August-University Goettingen

Third Member of the Thesis Committee

Prof. Dr. Jutta Gärtner

Department of Neuropediatrics

University Medical Center, Georg-August-University Goettingen

Date of disputation: May $7^{\text {th }}, 2014$ 
Meinem lieben

Ehemann Stephan 


\section{Affidavit}

I hereby declare that my dissertation entitled "Analyzing the effects of laquinimod on innate and adaptive immunity in mice with experimental autoimmune encephalomyelitis" has been written independently with no other sources and aids than quoted.

\section{Mativa Ot}

Martina Ott

Goettingen, March 2014 


\title{
Publications
}

\author{
Original Article
}

Ribes S, Meister T, Ott M, Redlich S, Janova H, Hanisch UK, Nessler S, Nau R

Intraperitoneal prophylaxis with CpG oligodeoxynucleotides protects neutropenic mice against intracerebral Escherichia coli K1 infection

J Neuroinflammation 2014, 11:14

\section{Abstracts}

Wegner C, Ott M, Hayardeny L, Lund BT, Nessler S, Brück W

Laquinimod induces a shift in natural killer cell populations in mice with experimental autoimmune encephalomyelitis

$29^{\text {th }}$ Congress of the European Committee for Treatment and Research in Multiple Sclerosis, October 2-5 ${ }^{\text {th }}$ 2013, Copenhagen, Denmark, Postersession

Ott M, Wegner C, Nessler S, Brück W

Preventive treatment with laquinimod reduces myeloid dendritic cells and shifts proinflammatory to regulatory $\mathrm{T}$ cells in experimental autoimmune encephalomyelitis

$11^{\text {th }}$ Congress of the International Society of Neuroimmunology, November $4-8^{\text {th }} 2012$, Boston, USA, Postersession

Ott M, Wegner C, Nessler S, Brück W

Preventive laquinimod treatment shifts pro-inflammatory to regulatory $\mathrm{T}$ cells and reduces myeloid dendritic cells in experimental autoimmune encephalomyelitis

$28^{\text {th }}$ Congress of the European Committee for Treatment and Research in Multiple Sclerosis, October $10-13^{\text {th }} 2012$, Lyon, France, Postersession 


\section{Contents}

Acknowledgements

Abstract III

List of figures $\quad$ V

$\begin{array}{lc}\text { List of formulas } & \text { VI }\end{array}$

$\begin{array}{ll}\text { List of tables } & \text { VII }\end{array}$

$\begin{array}{lll}\text { Abbreviations } & \text { VIII }\end{array}$

\section{Introduction}

$\begin{array}{llr}1.1 & \text { Multiple sclerosis } & 1\end{array}$

$\begin{array}{ll}\text { 1.1.1 Clinical course and diagnostics } & 1\end{array}$

$\begin{array}{ll}\text { 1.1.2 Epidemiology and etiology } & 2\end{array}$

$\begin{array}{ll}\text { 1.1.3 Pathogenesis } & 4\end{array}$

$\begin{array}{lll}\text { 1.1.4 Pathology } & 5\end{array}$

$\begin{array}{llr}1.1 .5 & \text { Treatment } & 7\end{array}$

$\begin{array}{llr}1.2 & \text { Experimental autoimmune encephalomyelitis } & 9\end{array}$

$\begin{array}{llr}\text { 1.2.1 } & \mathrm{EAE} \text { induction } & 9\end{array}$

$\begin{array}{ll}\text { 1.2.2 Effector cells in } \text { MOG }_{35-55} \text {-induced EAE } & 10\end{array}$

1.2.3 The value of EAE for the development of MS therapies 11

$\begin{array}{llr}1.3 & \text { Natural killer cells } & 13\end{array}$

$\begin{array}{lll}\text { 1.3.1 NK cell biology } & 13\end{array}$

$\begin{array}{ll}\text { 1.3.2 NK cells and autoimmunity } & 15\end{array}$

1.3.3 NK cells in the treatment of multiple sclerosis 16

$\begin{array}{ll}1.4 & \text { The new immunomodulator laquinimod } \\ \end{array}$

$\begin{array}{ll}\text { 1.4.1 Clinical studies } & 17\end{array}$

$\begin{array}{llr}\text { 1.4.2 } & \text { Experimental studies } & 18\end{array}$

$\begin{array}{lll}\text { 1.4.2.1 Effects of LAQ within the CNS } & 19\end{array}$

$\begin{array}{ll}\text { 1.4.2.2 LAQ effects on immune cells } & 19\end{array}$ 


\section{Materials and methods}

$\begin{array}{llr}2.1 & \text { Materials } & 22\end{array}$

$\begin{array}{lll}2.1 .1 & \text { Reagents } & 22\end{array}$

2.1.2 Solutions, buffers and cell culture media 23

2.1.3 Cell lines and bacteria 25

$\begin{array}{lll}\text { 2.1.4 Proteins, cytokines and dyes } & 25\end{array}$

$\begin{array}{lll}2.1 .5 & \text { Applied kits } & 29\end{array}$

$\begin{array}{ll}\text { 2.1.6 Oligonucleotide primers } & 29\end{array}$

$\begin{array}{lll}2.1 .7 & \text { Consumables } & 30\end{array}$

$\begin{array}{lll}2.1 .8 & \text { Technical devices } & 31\end{array}$

$\begin{array}{lll}2.1 .9 & \text { Software } & 31\end{array}$

$\begin{array}{lll}2.2 & \text { Animals } & 32\end{array}$

2.2.1 Mouse strains 32

$\begin{array}{lll}2.2 .2 & \text { Breeding } & 32\end{array}$

$\begin{array}{lll}2.2 .3 & \text { Housing } & 33\end{array}$

$\begin{array}{lll}2.3 & \text { Methods } & 33\end{array}$

2.3.1 Genotyping of genetically modified mice 33

2.3.2 Experimental autoimmune encephalomyelitis 35

$\begin{array}{lll}\text { 2.3.2.1 EAE induction } & 36\end{array}$

2.3.2.2 Clinical EAE score 36

2.3.2.3 LAQ treatment 36

$\begin{array}{lll}\text { 2.3.2.4 NK cell depletion in EAE } & 37\end{array}$

2.3.3 Analysis of immune cells ex vivo 37

$\begin{array}{lll}\text { 2.3.3.1 Preparation of single cell suspensions } & 38\end{array}$

$\begin{array}{lll}\text { 2.3.3.1.1 Preparation of spleen leukocytes } & 38\end{array}$

2.3.3.1.2 Preparation of leukocytes from the lymph nodes 38

2.3.3.1.3 Preparation of peripheral blood leukocytes 39 
$\begin{array}{lll}\text { 2.3.3.2 Flow cytometry } & 39\end{array}$

2.3.3.2.1 FACS staining procedure of splenocytes $\quad 40$

2.3.3.2.2 FACS staining procedure of peripheral blood leukocytes $\quad 40$

2.3.3.2.3 Intracellular detection of FoxP3 40

2.3.3.2.4 Intracellular detection of cytokines 41

2.3.3.2.5 Classification T cells, dendritic cells and NK cells according to surface and intracellular markers

2.3.4 Co-culture experiments

2.3.4.1 Purification of NK cells 43

2.3.4.2 Purification of CD4+ T cells 44

2.3.4.3 Generation of bone marrow-derived dendritic cells 44

2.3.4.4 Culture of B16F10 cells 45

2.3.4.5 Co-culture of NK cells with B16F10 cells 46

2.3.4.6 Co-culture of NK cells with bone marrow-derived dendritic cells 46

2.3.4.7 Assessment of NK cell mediated lysis of B16F10 cells and dendritic cells by $\begin{array}{ll}\text { crystal violet staining } & 46\end{array}$

2.3.4.8 Co-culture of NK cells with CD4+ T cells 47

2.3.4.9 Co-culture of NK cells with bone marrow-derived dendritic cells and CD4+ T cells $\quad 47$

$\begin{array}{lll}\text { 2.3.4.10 Transwell experiments } & 48\end{array}$

2.3.4.11 Analysis of T cell proliferation by CFSE dilution 49

2.3.4.12 Detection of apoptosis via Annexin V and 7-AAD staining 49

$\begin{array}{ll}\text { 2.3.5 In vitro stimulation of cytokine secretion } & 50\end{array}$

$\begin{array}{lll}\text { 2.3.5.1 Stimulation of T cells } & 50\end{array}$

$\begin{array}{ll}\text { 2.3.5.2 Stimulation of NK cells } & 50\end{array}$

$\begin{array}{lll}\text { 2.3.5.3 Detection of cytokines using ELISA } & 51\end{array}$

2.3.6 In vitro LAQ treatment 51

2.3.6.1 In vitro LAQ treatment of T cells 51 
2.3.6.2 Intracellular detection of pro-inflammatory cytokines in T cells after in vitro LAQ treatment 51

2.3.6.3 In vitro LAQ treatment of bone marrow-derived dendritic cells 52

2.3.6.4 In vitro LAQ treatment of NK cells 52

2.3.6.5 Co-culture of LAQ pre-treated bone marrow-derived dendritic cells with NK cells 52

2.3.6.6 Assessment of NK cell activation by CD69 staining 53

2.3.7 Data analysis and statistics 53

\section{Results}

3.1 Preventive LAQ treatment blocked EAE induction and the development of autoreactive T cells in vivo

3.1.1 Preventive LAQ treatment completely inhibited acute EAE in $\mathrm{C57BI} / 6 \mathrm{~J}$ mice

3.1.2 LAQ treatment increased naïve and decreased effector memory T cells

3.1.3 LAQ treatment decreased pro-inflammatory $T$ cell subsets

3.1.3.1 LAQ reduced IL-17-producing CD4+ and $\gamma \delta \mathrm{T}$ cells

3.1.3.2 LAQ reduced IFN $\mathrm{L}$-producing CD4+ T cells

3.1.4 LAQ treatment increased the frequency of FoxP3+ regulatory T cells 58

3.1.5 LAQ had no direct effect on IL-17+ and IFN ++ CD4+ T cells in vitro

3.2 Preventive LAQ treatment decreased total dendritic cells and myeloid dendritic cells in vivo

3.2.1 LAQ treatment reduced the frequency of dendritic cells

3.2.2 LAQ treatment decreased myeloid dendritic cells

3.3 Preventive LAQ treatment led to the activation of natural killer cells in vivo

3.3.1 LAQ treatment reduced absolute NK cell numbers, but did not alter NK cell frequencies

3.3.2 LAQ increased activation markers on the surface of NK cells

3.3.2.1 LAQ treatment shifted NK cell subsets towards more CD27+ NK cells 
3.3.2.2.2 LAQ upregulated activating NK cell receptors

3.3.2.2.3 LAQ downregulated inhibitory NK cell receptors 69

3.3.3 In vivo LAQ treatment increased NK cell effector functions ex vivo 73

3.3.3.1 LAQ enhanced the killing efficiency of NK cells towards tumor cells in vitro $\quad 73$

3.3.3.2 LAQ increased IFNY-producing NK cells upon stimulation ex vivo 75

3.4 In vivo LAQ-treated NK cells inhibited antigen-dependent T cell proliferation in vitro in a contact dependent manner

3.4.1 In vivo LAQ-treated NK cells reduced antigen-dependent T cell proliferation in a triple co-culture system of NK cells, T cells and bone marrow-derived dendritic cells by direct cell contact

3.4.1.1 LAQ-treated NK cells reduced $\mathrm{MOG}_{35-55}$-induced T cell proliferation

3.4.1.2 The NK cell-mediated inhibition of $\mathrm{MOG}_{35-55}$-induced $\mathrm{T}$ cell proliferation was dependent on direct cell contact

3.4.1.3 NK cells induced cell death in T cells and bone marrow-derived dendritic cells irrespective of in vivo LAQ treatment

3.4.2 NK cells did not affect antigen-independent $T$ cell proliferation in a co-culture system with T cells

3.4.3 NK cells did not kill bone marrow-derived dendritic cells in a co-culture system

3.5 LAQ-mediated effects on NK cells were independent of dendritic cells and vice versa

3.5.1 NK cell and dendritic cell responses to LAQ treatment preceded changes in the T cell compartment

3.5.2 The effect of LAQ on NK cells was independent of T and B cells in vivo

3.5.3 NK cell depletion did not affect the dendritic cell phenotype after LAQ treatment in vivo

3.5.4 LAQ directly activated NK cells in vitro

\subsection{NK cell depletion reduced the therapeutic efficiency of LAQ in EAE}

3.6.1 NK cell depletion was efficient in Th/+ mice

3.6.2 NK cell depletion exacerbated EAE symptoms in LAQ-treated animals 


\section{Discussion}

4.1 Preventive LAQ treatment inhibited EAE and reduced dendritic cells and proinflammatory T cells in vivo

4.2 Preventive LAQ treatment induced NK cell activation in vivo

4.2.1 LAQ did not alter splenic NK cell frequencies

4.2.2 LAQ treatment led to a relative expansion of the CD27+ NK cell subset

4.2.3 LAQ activated NK cells

4.2.4 LAQ enhanced NK cell effector functions

4.2.5 Human NK cells in MS - a potential target for LAQ treatment?

4.3 LAQ-treated NK cells inhibited antigen-specific $\mathrm{T}$ cell proliferation in a contactdependent manner

4.4 LAQ exerted independent effects on NK cells and dendritic cells

4.4.1 LAQ-mediated changes displayed a similar kinetic in NK cells and dendritic cells and were delayed in T cells

4.4.2 The effects of LAQ on dendritic cells were independent of NK cells in vivo

4.4.3 LAQ treatment directly induced NK cell activation

4.4.4 LAQ interferes with different signaling pathways depending on the target cell

4.5 NK cell depletion decreased the therapeutic efficiency of LAQ in EAE

4.6 Outlook 


\section{Acknowledgements}

First and foremost I would like to thank my supervisors Dr. Dr. Christiane TheodossiouWegner and Dr. Stefan Nessler for the guidance and the great support during the years of my PhD thesis and for sharing their scientific expertise with me. I am particularly grateful for the opportunity to work independently and to develop own ideas. Thank you very much for believing in me and for always being open for discussions, questions and problems.

I would also like to express my deep gratitude to Prof. Dr. Wolfgang Brück, for giving me the opportunity to work on this interesting project in his lab, for the supervision of the project and for his scientific input and discussions.

In addition, I want to thank Prof. Dr. Christine Stadelmann-Nessler for always having an open ear for any kind of problems and for giving scientific as well as personal advice.

Moreover, I am thankful to the members of my thesis committee Prof. Dr. Holger Reichardt and Prof. Dr. Jutta Gärtner for the comments and the fruitful discussions during my progress reports. I would additionally like to thank Prof. Dr. Holger Reichardt for agreeing to act as second examiner of my PhD thesis. Further thanks to the additional members of my examination board Prof. Dr. Martin Weber, Dr. Francesca Odoardi and Dr. Tobias Pukrop.

I would like to especially thank our collaboration partner Prof. Dr. Evelyn Ullrich (Children's Hospital, Department of Pediatric Hematology and Oncology, Goethe-University, Frankfurt, Germany) for giving me the opportunity to learn new methods in her lab, for providing the B16F10 melanoma cell line and for her scientific input. I further want to acknowledge Dr. Kathrin Meinhardt (former lab member of Prof. Dr. Evelyn Ullrich) for methodological advice and discussions. A special thanks to Ms. Stephanie Gerstner (University Medicine Erlangen, Department of Medicine 5-Haematology and Oncology, Friedrich-Alexander-University ErlangenNuremberg, Germany) for her support with my NK cells and other problems and for being available to give advice, any time day or night.

Further, I want to thank Prof. Dr. Martin Weber and Ms. Silke Kinzel for providing part of the Th/+ mice, used in this study. 
I also want to acknowledge Mr. Markus Harden (Department for Medical Statistics, University Medical Center Goettingen) for performing the statistical analysis of the EAE score data in this work.

I owe many thanks to the "Stiftung der Deutschen Wirtschaft" for personal funding and non-material support in form of seminars and trainings.

I want to thank our technical assistants Mareike, Katja, Brigitte, Uta, Heidi, Jasmin, Elke and Susanne for valuable help and support and also for nice chats and a good time in the lab.

Special thanks to my colleagues Ramona, Franzi, Nadine, Lena, Claudia, Alonso, Anne, Nielsen, Patrik, Silke, Darius, Angie and all the other colleagues for help and advice, scientific discussions and of course for the emotional support and the great time inside and outside the lab.

Further, I want to acknowledge Cynthia, Chris and Julia for the administrative support and the PhD program Molecular Medicine, especially Dr. Eric Meskauskas.

Very personal thanks to my parents Erika and Gerhard and my sister Barbara for always supporting me.

Finally, I want to thank my husband Stephan for his endless patience and emotional support. 


\section{Abstract}

Laquinimod (LAQ), a new orally active immunomodulator, is currently under investigation for the treatment of multiple sclerosis (MS). In clinical trials LAQ considerably reduced the annualized relapse rate, disability progression and brain atrophy in MS patients, but its exact mechanism of action is not fully understood. Experimental data provide evidence for immunomodulatory effects of LAQ on antigen presenting cells and T cells, but the role of natural killer (NK) cells within the mechanism of action of LAQ has not been investigated yet.

The aim of the present study was to evaluate effects of LAQ on innate and adaptive immune cells in mice with experimental autoimmune encephalomyelitis (EAE), with a special focus on NK cells.

First, a detailed analysis of immune cells was performed in $\mathrm{MOG}_{35-55}$-immunized animals preventively treated with $25 \mathrm{mg} / \mathrm{kg} \mathrm{LAQ}$. Innate immune cells responded quickly to LAQ treatment, whereas major changes in the T cell compartment were detectable several days later. LAQ reduced the total number of splenic dendritic cells and the number of myeloid dendritic cells. In parallel, NK cells were activated by LAQ. As such LAQ increased the frequency of CD69+ NK cells and upregulated a number of activating NK cell receptors such as DNAM-1 and TACTILE. TIGIT, an inhibitory NK cell receptor, which shares the same ligand with DNAM-1 and TACTILE, was downregulated, suggesting that LAQ shifts the balance of inhibitory and activating NK cell receptors in favor of the latter.

To test, whether the LAQ-induced NK cell activation improves classical NK cell effector functions, NK cells were sorted ex vivo from LAQ- or vehicle-treated animals. NK cells derived from LAQ-treated mice killed B16F10 melanoma cells in vitro more efficiently than NK cells derived from vehicle controls and produced more cytokines in response to IL-12 and IL-18 stimulation. In a triple co-culture system with 2D2 T cells and bone marrow-derived dendritic cells, $\mathrm{T}$ cell proliferation in response to $\mathrm{MOG}_{35-55}$ was significantly better inhibited by NK cells derived from LAQ-treated mice in a cell contact-dependent manner.

Finally, LAQ efficacy was analyzed in NK cell-depleted and competent EAE animals. Th/+ mice were depleted by administration of PK-136 antibodies, immunized with $\mathrm{MOG}_{35-55}$ and preventively treated with $25 \mathrm{mg} / \mathrm{kg}$ LAQ. Treatment with LAQ markedly attenuated EAE severity in NK cell-competent Th/+ mice. This beneficial LAQ effect was however only transient in Th/+ mice depleted of NK cells. While LAQ treatment prevented the death of animals in the presence of NK cells, a considerable number of animals depleted of NK cells died despite LAQ therapy. 
In conclusion, this study identified NK cells as a novel direct cellular target of LAQ therapy with a central relevance for the therapeutic efficiency of the substance in EAE. These data indicate that the LAQ-mediated activation of NK cells is a crucial part of the immunomodulatory mechanism of LAQ in vivo. Most interestingly, LAQ specifically interfered with the DNAM1/TACTILE/TIGIT pairwise receptor family on NK cells, favoring a central role of this pathway within the mechanism of action of LAQ. It remains to be investigated whether similar effects of LAQ on human NK cells can be detected in patients with MS. Since published data reveal defective NK cell function as one pathogenic factor in MS, LAQ-induced restoration of NK cell functions could be a major component of its protective mechanisms in the treatment of MS patients. 


\section{List of figures}

\begin{tabular}{|c|c|}
\hline FIGURE 1 & Structural formula of laquinimod \\
\hline FIGURE 2 & $\mathrm{LAQ}$ treatment protects $\mathrm{C} 57 \mathrm{BI} / 6 \mathrm{~J}$ mice from $\mathrm{MOG}_{35-55}$-induced $\mathrm{EAE}$ \\
\hline FIGURE 3 & LAQ augments naïve and reduces effector memory T cells \\
\hline FIGURE 4 & In vivo LAQ treatment reduces Th17 cells and IL-17-producing $\gamma \delta$ T cells \\
\hline FIGURE 5 & LAQ therapy reduces Th1 cells \\
\hline FIGURE 6 & $\begin{array}{l}\text { Regulatory T cells are increased in naïve and immunized mice after LAQ } \\
\text { treatment }\end{array}$ \\
\hline FIGURE 7 & LAQ has no direct effect on Th17 and Th1 cells in vitro \\
\hline FIGURE 8 & LAQ decreases the frequency of dendritic cells in the spleen \\
\hline FIGURE 9 & LAQ reduces myeloid dendritic cells in the spleen \\
\hline FIGURE 10 & $\begin{array}{l}\text { LAQ reduces absolute NK cell numbers, but does not alter NK cell } \\
\text { frequencies }\end{array}$ \\
\hline FIGURE 11 & LAQ interferes with the relative frequency of NK cell subpopulations \\
\hline FIGURE 12 & LAQ augments the proportion of CD69+ NK cells \\
\hline FIGURE 13 & LAQ increases the expression of activating NK cell receptors \\
\hline FIGURE 14 & Effects of LAQ on the expression of TACTILE and DNAM-1 on T cells \\
\hline FIGURE 15 & LAQ reduces the expression of inhibitory NK cell receptors \\
\hline FIGURE 16 & LAQ does not regulate TIGIT on T cells \\
\hline FIGURE 17 & LAQ increases the NK cell-mediated killing of tumor cells in vitro \\
\hline FIGURE 18 & LAQ increases IFN $\gamma+\mathrm{NK}$ cells \\
\hline FIGURE 19 & LAQ-treated NK cells inhibit T cell proliferation \\
\hline FIGURE 20 & NK cell-mediated inhibition of T cell proliferation is contact-dependent \\
\hline FIGURE 21 & NK cells induce cell death in T cells in the triple co-culture system \\
\hline FIGURE 22 & NK cells induce cell death in dendritic cells in the triple co-culture system \\
\hline FIGURE 23 & $\begin{array}{l}\text { NK cells do not interfere with T cell proliferation, when co-cultured with T } \\
\text { cells alone }\end{array}$ \\
\hline FIGURE 24 & NK cells do not kill dendritic cells when co-cultured with dendritic cells alone \\
\hline FIGURE 25 & $\begin{array}{l}\text { LAQ alters surface marker expression on NK cells after one to two days of } \\
\text { treatment }\end{array}$ \\
\hline FIGURE 26 & $\begin{array}{l}\text { LAQ reduces total dendritic cells and leads to upregulation of the co- } \\
\text { stimulatory molecules CD80 and CD86 after two to three days of treatment }\end{array}$ \\
\hline FIGURE 27 & No effects of LAQ on T cells after four days of treatment \\
\hline
\end{tabular}




\begin{tabular}{l|lr} 
FIGURE 28 & T and B cells are dispensable for the LAQ effect on NK cells & 96 \\
FIGURE 29 & The effects of LAQ on dendritic cells are not mediated by NK cells & 98 \\
FIGURE 30 & LAQ directly modulates NK cells in vitro & 99 \\
FIGURE 31 & Experimental setup for NK cell depletion and LAQ treatment in immunized & 101 \\
& Th/+ mice & 102 \\
FIGURE 32 & NK cell depletion is efficient & 103 \\
FIGURE 33 & LAQ-mediated reduction of EAE severity is dependent on NK cells & 104 \\
FIGURE 34 & NK cell depletion attenuates the therapeutic LAQ effect in EAE & 106 \\
FIGURE 35 & LAQ treatment is efficient in Th/+ mice and NK cell depletion does not alter & 107 \\
& EAE severity in controls & 109 \\
FIGURE 36 & NK cell depletion reduces the survival of LAQ-treated mice \\
FIGURE 37 & Summary of the effects of LAQ treatment on NK cells, dendritic cells and T
\end{tabular}

\section{List of formulas}

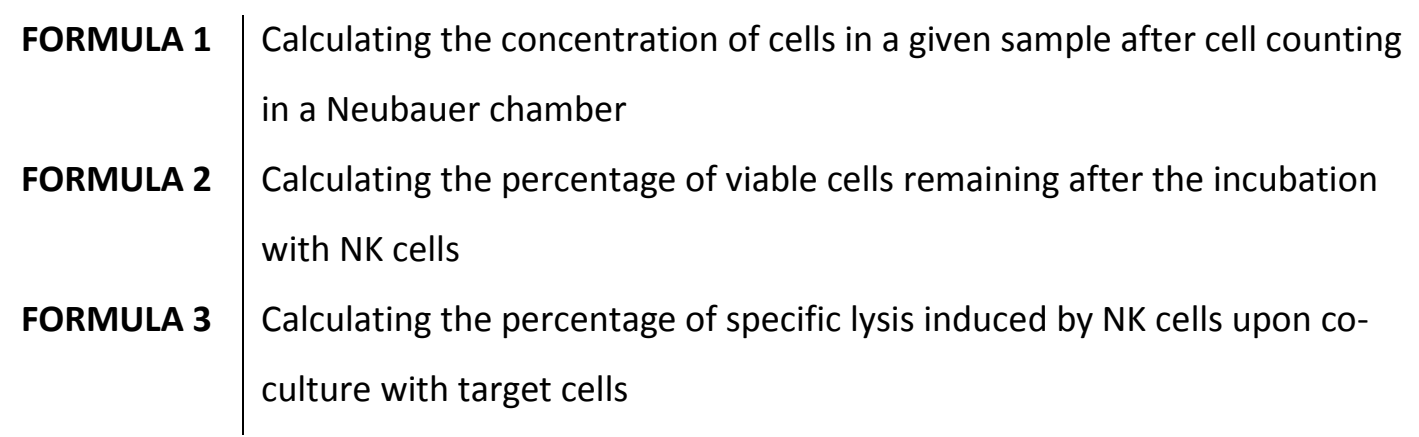




\section{List of tables}

\begin{tabular}{|c|c|}
\hline TABLE 1 & Reagents \\
\hline TABLE 2 & Solutions, buffers and cell culture media \\
\hline TABLE 3 & Cell lines and bacteria \\
\hline TABLE 4 & Proteins, enzymes and inhibitors \\
\hline TABLE 5 & Monoclonal antibodies for flow cytometry \\
\hline TABLE 6 & $\begin{array}{l}\text { Monoclonal antibodies for antigen-independent activation of T cell } \\
\text { proliferation in vitro }\end{array}$ \\
\hline TABLE 7 & Monoclonal antibodies for NK cell depletion in vivo \\
\hline TABLE 8 & Cytokines \\
\hline TABLE 9 & Fluorescent dyes \\
\hline TABLE 10 & Kits \\
\hline TABLE 11 & Oligonucleotide primers \\
\hline TABLE 12 & Consumables \\
\hline TABLE 13 & Technical devices \\
\hline TABLE 14 & Software \\
\hline TABLE 15 & Clinical EAE score \\
\hline TABLE 16 & Classification of T cell subpopulations \\
\hline TABLE 17 & Classification of dendritic cell subpopulations \\
\hline TABLE 18 & Classification of NK cell subpopulations \\
\hline TABLE 19 & $\begin{array}{l}\text { CD11b+ NK cells display higher cytotoxicity towards B16F10 cells in vitro } \\
\text { than CD27+ NK cells at all effector/target ratios }\end{array}$ \\
\hline TABLE 20 & $\begin{array}{l}\text { NK cells from LAQ-treated animals inhibit antigen-dependent T cell } \\
\text { proliferation in vitro }\end{array}$ \\
\hline TABLE 21 & $\begin{array}{l}\text { NK cells inhibit } \mathrm{MOG}_{35-55} \text {-induced T cell proliferation in a contact-dependent } \\
\text { way }\end{array}$ \\
\hline TABLE 22 & $\begin{array}{l}\text { NK cells trigger cell death in T cells and bone marrow-derived dendritic cells } \\
\text { independent of LAQ treatment in vivo }\end{array}$ \\
\hline TABLE 23 & $\begin{array}{l}\text { LAQ leads to upregulation of the activation-associated molecules CD69, } \\
\text { TACTILE and NKG2D on NK cells after one to two days of treatment }\end{array}$ \\
\hline TABLE 24 & $\begin{array}{l}\text { In vivo LAQ treatment reduces the total frequency of splenic dendritic cells } \\
\text { within three days and upregulates the co-stimulatory molecules CD80 and } \\
\text { CD86 within two to three days }\end{array}$ \\
\hline
\end{tabular}




\section{Abbreviations}

2D2

7-AAD

A

ADCC

AKT

ANOVA

APC

BBB

BD

BDNF

BL

bmDC

bp

BSA

${ }^{\circ} \mathrm{C}$

C

$\mathrm{Ca}^{2+}$

CCL21

CCR7

CD

CFA

CFSE

Cl

cm

CNS

$\mathrm{CO}_{2}$

CSF

Ctrl

CX3CR1

CXCR1

CXCR3

Cy7
MOG-specific T cell receptor transgenic mice

7-aminoactinomycin

Adenine

Antibody dependent cellular cytotoxicity

Protein kinase B

Analysis of variance

Allophycocyanin

Blood brain barrier

BD Biosciences, Franklin Lakes, NJ, USA

Brain-derived neurotrophic factor

BioLegend, San Diego, CA, USA

Bone marrow-derived dendritic cell

Base pairs

Bovine serum albumin

Degree Celsius

Cytosine

Calcium ion

Chemokine ( $\mathrm{CC}$ motif) ligand 21

Chemokine (CC motif) receptor 7

Cluster of differentiation

Complete Freund's adjuvant

Carboxyfluoresceinsuccinimidylester

Chloride

Centimeter

Central nervous system

Carbon dioxide

Cerebrospinal fluid

Control

Chemokine ( $\mathrm{CX} 3 \mathrm{C}$ motif) receptor 1

Chemokine (CXC motif) receptor 1

Chemokine (CXC motif) receptor 3

Cyanine 7 


\begin{tabular}{|c|c|}
\hline DIS & Disseminate in space \\
\hline DIT & Disseminate in time \\
\hline DMSO & Dimethyl sulfoxide \\
\hline DNA & Deoxyribonucleic acid \\
\hline DNAM-1 & DNAX Accessory Molecule-1 \\
\hline dNTP & Desoxynucleoside triphosphate \\
\hline EAE & Experimental autoimmune encephalomyelitis \\
\hline eB & eBioscience, San Diego, CA, USA \\
\hline EBV & Epstein-Barr virus \\
\hline EDTA & Ethylenediamine tetraacetic acid disodiumsalt dihydrate \\
\hline ELISA & Enzyme linked immunosorbent assay \\
\hline FACS & Fluorescence-activated cell sorting \\
\hline FCS & Fetal calf serum \\
\hline FITC & Fluorescein isothiocyanate \\
\hline FoxP3 & Forkhead box P3 \\
\hline G & Guanine \\
\hline g & Gram \\
\hline$g$ & Gravitation acceleration \\
\hline $\mathbf{G}_{0}$ & Gap 0 \\
\hline $\mathbf{G}_{1}$ & Gap 1 \\
\hline GA & Glatiramer acetate \\
\hline GdE & Gandolinium-enhancing \\
\hline GM-CSF & Granulocyte macrophage colony-stimulating factor \\
\hline GWAS & Genome wide association study \\
\hline H & Hydrogen \\
\hline h & Hour(s) \\
\hline$H-60$ & Heat shock protein 60 \\
\hline HBSS & Hank's buffered salt solution \\
\hline $\mathrm{HCl}$ & Hydochloric acid \\
\hline HEPES & 4-(2-hydroxyethyl)-1-piperazineethanesulfonic acid \\
\hline HLA & Human leukocyte antigen \\
\hline i.p. & Intraperitoneal \\
\hline IFN $\beta$ & Interferon-beta \\
\hline IFNp & Interferon-gamma \\
\hline IgG & Immunoglobulin G \\
\hline
\end{tabular}




\begin{tabular}{|c|c|}
\hline IgH chain & Immunoglobulin heavy chain \\
\hline IL & Interleukin \\
\hline ITAM & Immunoreceptor tyrosine-based activation motif \\
\hline ITIM & Immune-receptor tyrosine-based inhibitory motif \\
\hline JAK & Janus kinase \\
\hline JCV & John Cunningham virus \\
\hline JNK & c-Jun N-terminal kinase \\
\hline kg & Kilogram \\
\hline KIR & Killer cell immunoglobulin-like receptors \\
\hline LAQ & Laquinimod \\
\hline LFA-1 & Lymphocyte function-associated antigen 1 \\
\hline LPS & Lipopolysaccharide \\
\hline $\mathbf{M}$ & Molar \\
\hline MACS & Magnetic-activated cell sorting \\
\hline MAP kinase & Mitogen-activated protein kinase \\
\hline MBP & Myelin basic protein \\
\hline $\mathrm{MBP}_{1-9}$ & Myelin basic protein amino acids 1 - 9 \\
\hline MEM & Minimum essential medium \\
\hline mg & Milligram \\
\hline $\mathrm{Mg}^{2+}$ & Magnesium ion \\
\hline MHC & Major histocompatibility complex \\
\hline $\mathrm{MHCl}$ & Major histocompatibility complex class I molecule \\
\hline MHCII & Major histocompatibility complex class II molecule \\
\hline MICA & MHC class I polypeptide-related sequence $A$ \\
\hline MICB & MHC class I polypeptide-related sequence B \\
\hline $\min$ & Minute(s) \\
\hline ml & Milliliter \\
\hline mm & Millimeter \\
\hline $\mathrm{mM}$ & Millimolar \\
\hline MOG & Myelin oligodendrocyte glycoprotein \\
\hline MOG $_{1-22}$ & Myelin oligodendrocyte glycoprotein amino acids 1 - 22 \\
\hline MOG $_{35-55}$ & Myelin oligodendrocyte glycoprotein amino acids 35 - 55 \\
\hline MRI & Magnetic resonance imaging \\
\hline MRP14 & Myeloid-related protein 14 \\
\hline MS & Multiple sclerosis \\
\hline
\end{tabular}




\begin{tabular}{|c|c|}
\hline$\mu \mathrm{g}$ & Microgram \\
\hline$\mu l$ & Microliter \\
\hline$\mu \mathrm{m}$ & Micrometer \\
\hline$\mu \mathrm{M}$ & Micromolar \\
\hline $\mathbf{n}$ & Number of independent samples or animals \\
\hline $\mathrm{NaCl}$ & Sodium chloride \\
\hline NAWM & Normal appearing white matter \\
\hline NF-KB & Nuclear factor kappa B \\
\hline ng & Nanogram \\
\hline NK & Natural killer \\
\hline NKG2A & Natural killer group 2, member A \\
\hline NKG2D & Natural killer group 2, member D \\
\hline NKp30 & Natural killer cell p30-related protein \\
\hline NKp44 & Natural killer cell p44-related protein \\
\hline NKp46 & Natural killer cell p46-related protein \\
\hline NKp80 & Natural killer cell p80-related protein \\
\hline NKT & Natural killer T cell \\
\hline $\mathrm{nm}$ & Nanometer \\
\hline OD & Optical density \\
\hline ODC & Oligodendrocyte \\
\hline OSE & Optico-spinal experimental autoimmune encephalomyelitis \\
\hline OT-1 & Ovalbumin-specific T cell receptor \\
\hline OVA & Ovalbumin \\
\hline PBS & Phosphate buffered salt solution \\
\hline p.i. & Post immunization \\
\hline p21 & Cyclin-dependent kinase inhibitor 1 \\
\hline PCR & Polymerase chain reaction \\
\hline PE & Phycoerythrin \\
\hline PerCP & Peridinin chlorophyll protein \\
\hline pg & Picogram \\
\hline PLP & Proteolipid protein \\
\hline PLP $_{139-151}$ & Proteolipid protein amino acids $139-151$ \\
\hline PMA & Phorbol 12-myristate 13-acetate \\
\hline PML & Progressive multifocal leukoencephalopathy \\
\hline PPMS & Primary progressive multiple sclerosis \\
\hline
\end{tabular}




\begin{tabular}{|c|c|}
\hline PTX & Pertussis toxin \\
\hline RA & Rheumatoid arthritis \\
\hline RAE & Retinoic acid early-inducible protein \\
\hline Rag1 & Recombination activating gene 1 \\
\hline $\operatorname{Rag} 1^{-/-}$ & Rag1-deficient \\
\hline rpm & Rotations per minute \\
\hline RPMI-1640 & Roswell Park Memorial Institute-1640 \\
\hline RRMS & Relapsing remitting multiple sclerosis \\
\hline RT & Room temperature \\
\hline $\mathbf{s}$ & Second(s) \\
\hline S1P & Sphingosin-1-phosphat \\
\hline SDS & Sodium dodecyl sulfate \\
\hline SEM & Standard error of the mean \\
\hline SHP1 & SH2 domain-containing tyrosine phosphatase 1 \\
\hline SHP2 & SH2 domain-containing tyrosine phosphatase 2 \\
\hline SLE & Systemic lupus erythematosus \\
\hline SPF & Specific pathogen free \\
\hline SPMS & Secondary progressive multiple sclerosis \\
\hline STAT & Signal transducer and activator of transcription \\
\hline $\mathbf{T}$ & Thymine \\
\hline TACTILE & T cell-activated increased late expression \\
\hline TBE & Tris/borate/EDTA \\
\hline T-bet & T cell-specific T-box transcription factor \\
\hline TCR & T cell receptor \\
\hline Th & MOG-specific B cell receptor transgenic mice \\
\hline Th/+ & Heterozygous MOG-specific B cell receptor transgenic mice \\
\hline Th1 & T helper 1 \\
\hline Th17 & T helper 17 \\
\hline Th2 & Thelper 2 \\
\hline TID & Type I diabetes \\
\hline TIGIT & T cell immunoreceptor with Ig and ITIM domains \\
\hline TMB & $3,3^{\prime}, 5,5^{\prime}$-tetramethylbenzidine \\
\hline TNF & Tumor necrosis factor \\
\hline TRAIL & TNF-related apoptosis-inducing ligand \\
\hline Treg & Regulatory $\mathrm{T}$ cell \\
\hline
\end{tabular}




\begin{tabular}{l|l} 
Tris & Tris(hydroxymethyl)aminomethane \\
$\mathbf{U}$ & Units \\
ULBP & UL-16 binding protein \\
$\mathbf{U V}$ & Ultra violet \\
$\mathbf{V}$ & Volt \\
VCAM-1 & Vascular cell adhesion molecule-1 \\
VLA-4 & Very late antigen-4 \\
V $\boldsymbol{\alpha}$ & Variable alpha chain \\
V $\boldsymbol{\beta}$ & Variable beta chain \\
$\boldsymbol{\infty}$ & Infinite
\end{tabular}




\section{Introduction}

\subsection{Multiple sclerosis}

Multiple sclerosis (MS), also known as encephalomyelitis disseminata, is a chronic inflammatory demyelinating disease of the central nervous system (CNS) with typically multifocal presentation. The disease course and symptoms show a broad range between individual patients and the etiology of MS is still not fully understood. MS is typically classified as an autoimmune disease and the destruction of CNS-intrinsic structures by auto-reactive immune cells appears to be one important mechanism leading to CNS injury. To date, ten medications have been approved for the long term disease-modifying treatment of MS, which display immunomodulatory or immunosuppressive properties and mainly target the autoimmune component of the disease. Additionally, several promising immunomodulatory drugs are currently under investigation for the treatment of MS.

\subsubsection{Clinical course and diagnostics}

MS is the most common non-traumatic neurological disease which leads to chronic disability in young adults. Generally two different forms can be distinguished based on the disease course at the beginning of the disease: relapsing remitting and primary progressive MS.

Relapsing remitting MS (RRMS) is the most common form of MS in the early phase of disease, initially affecting $80-85 \%$ of MS patients. RRMS is characterized by the occurrence of relapses and an early onset of disease (mean 29 years). Typically, women display a two to three fold increased risk to develop RRMS compared to men and the most common initial symptoms are visual and sensory symptoms. A relapse is defined as an episode of neurological symptoms typical for an inflammatory demyelinating event in the CNS that lasts for at least 24 hours in the absence of fever and infection (Polman et al., 2011). After a relapse, patients typically show complete or partial recovery from the neurological symptoms. Up to now, ten medications have been approved for the long-term treatment of RRMS.

At later disease stages about two thirds of RRMS patients develop a progressive disease course, referred to as secondary progressive MS (SPMS). Typically patients with SPMS do not experience episodes of acute symptoms with subsequent recovery anymore, but instead accumulate neurological deficits in a chronic process.

$10-15 \%$ of MS patients initially present with gradually increasing disability in the absence of relapses, a disease course known as primary progressive MS (PPMS). In contrast to RRMS, 
PPMS equally affects men and women and is typically associated with a relatively late disease onset (mean 40 years). Characteristic neurological deficits are progressive spastic paresis and leg weakness, which are frequently caused by lesions in the spinal cord. Magnetic resonance imaging (MRI) typically reveals less distinct white matter lesions compared to RRMS patients. Currently, no efficient therapeutic strategy is available for the treatment of PPMS patients.

There is a broad spectrum of neurological symptoms during a relapse, which depends on the affected CNS region. Typical symptoms caused by lesions within the optic nerve are visual symptoms such as blurred vision. Brain stem lesions can lead to double vision. Furthermore, sensory deficits, like paresthesia, pain and sensible ataxia, and motor symptoms, like para-, tetraor hemiplegia, commonly occur. These symptoms can be associated with cerebral as well as spinal lesions, the latter typically leading to more severe neurological deficits. Bladder dysfunction is a frequent vegetative symptom. In addition, MS patients can experience cerebellar dysfunction such as dysmetria and ataxia. Affective disorders and fatigue are common, too. Cognitive deficits are observed very frequently in about $40 \%$ of the patients during the course of disease (Rao et al., 1991).

MS is classically diagnosed using the McDonalds criteria, which were implemented in 2000 (McDonald et al., 2001) and revised in 2005 (Polman et al., 2005) and 2011 (Polman et al., 2011). The diagnosis is based on three factors: clinical symptoms, MRI data and cerebrospinal fluid (CSF) parameters. Concerning MRI data, lesions have to show dissemination in space (DIS) and time (DIT) to be considered predictive for MS. According to the current McDonalds criteria DIS is defined as at least one T2 lesion appearing in at least two of the four regions characteristic for MS: juxtacortical, periventricular, infratentorial and the spinal cord. The criterion of DIT is fulfilled when asymptomatic gadolinium-enhancing ( $\mathrm{GdE}$ ) and non-enhancing lesions are simultaneously present in one single MRI scan or when a new T2 or GdE lesion is detected on a follow up MRI. CSF markers are mainly used for the diagnosis of PPMS (Polman et al., 2011). Typical CSF parameters for MS include a slight mononuclear pleocytosis, elevated immunoglobulin G (IgG) concentrations and oligoclonal bands.

\subsubsection{Epidemiology and etiology}

MS is a common neurological disease affecting approximately 2.5 million patients worldwide (Milo and Kahana, 2010). MS displays an unequal geographic distribution, whereby the risk to develop MS appears to increase with the distance to the equator. High risk regions comprise Europe, Russia, Israel, Northern US, Canada and New Zealand (Kurtzke, 2000). In Germany, the prevalence of MS is relatively high with 127 patients per 100.000 inhabitants (Hein and Hopfenmuller, 2000). Besides geographic aspects, gender is a risk factor for RRMS. Within the 
Caucasian population the risk to develop RRMS is two to three times higher in females than in males and this ratio is increasing over time (Kurtzke, 2000, Orton et al., 2010, Ahlgren et al., 2011, Wallin et al., 2012). This observation led to the concept that sex hormones could be implicated in the pathogenesis of MS and indeed female hormones seem to influence disease activity during pregnancy and estriol substitution attenuates MRI activity (Confavreux et al., 1998, Soldan et al., 2003).

It is widely accepted that MS is an autoimmune disorder. This assumption is based on genome wide association studies which demonstrated that the genetic variants, which are relevant for MS, belong to the function of the immune system. In addition, therapies which modulate or suppress the immune system are effective at least in RRMS. MS is most likely caused by a complex interplay between multiple predisposing genes and environmental factors. A genetic component of MS was indicated by twin studies, which revealed $25 \%$ concordance for homozygotic twins and 3 - 5\% concordance for dizygotic twins and other first degree relatives (Mumford et al., 1994, Willer et al., 2003, Hansen et al., 2005). On the other hand, migration studies argue for the involvement of environmental factors (Dean and Kurtzke, 1971).

In the 1970's the first risk genes for MS, the human leukocyte antigen (HLA) genes, were described. Currently several HLA class II haplotypes are known to be associated with increased susceptibility for MS, like DRB1*15:01, DQA1*01:02 and DQB*06:02, with DRB1*15:01 showing the strongest association (Munoz-Culla et al., 2013). In an attempt to thoroughly investigate the risk genes for MS, a large international genome wide association (GWAS) study was performed by the International Multiple Sclerosis Genetics Consortium and the Wellcome Trust Case Control Consortium 2. The GWAS study identified 29 susceptibility genes, which include, besides the well known HLA genes, mainly risk genes playing a role in immunity and T cell differentiation, like genes associated with cytokine signaling (IL2RA, IL7R, IL12A), co-stimulation (CD40, CD80, CD86) and signal transduction (MALT1, STAT3). These findings consolidate the crucial role of the immune system in the pathogenesis of MS (Sawcer et al., 2011).

Several environmental factors influence the risk to develop MS. Low vitamin D intake and serum levels are associated with an increased risk to develop MS and high vitamin D serum levels reduce the relapse risk (Munger et al., 2004, Munger et al., 2006, Simpson et al., 2010, Lucas et al., 2011). The critical role of vitamin D is further supported by data from the GWAS study, identifying CYP27B1 as a risk gene, which encodes a crucial enzyme for the conversion of vitamin D into its active form (Sawcer et al., 2011, Hewer et al., 2013). Furthermore, Epstein-Barr virus (EBV) infection has been discussed as potentially relevant environmental risk factor, but dissecting the role of EBV infection in the development of MS in adults is difficult, due to the high seroprevalence of EBV infection in more than $90 \%$ of the adult population (Ascherio and Munger, 
2007, Almohmeed et al., 2013). Moreover, cigarette smoking is associated with an 1.5 fold increased risk to develop MS, clinical worsening and a faster transition from RRMS to SPMS (hazard ratio 2.5) (Hernan et al., 2001, Healy et al., 2009, Hedstrom et al., 2009).

\subsubsection{Pathogenesis}

MS is generally classified as chronic inflammatory autoimmune disease of the CNS. Especially during the early phase of MS, infiltration of auto-reactive immune cells is thought to be the primary cause of demyelination and CNS damage.

The following sequence of events was described: Auto-reactive $T$ cells become activated by an unknown trigger in the periphery. This leads to the upregulation of adhesion molecules on the surface of activated $T$ cells and enables them to interact with endothelial cells, which promotes the adhesion and transmigration of activated T cells through the blood-brain barrier (BBB). Within the CNS, T cells become locally reactivated by antigen presenting cells and start to produce pro-inflammatory cytokines, especially interferon-gamma (IFNY) and interleukin 17 (IL17). Pro-inflammatory cytokines and chemokines in turn activate resident microglia, increase the permeability of the BBB and recruit further inflammatory cells, subsequently causing demyelination and tissue damage (Sospedra and Martin, 2005, Buc, 2013).

A dense infiltrate of $T$ cells can be detected in active MS lesions, consisting of mainly CD8+, but also of CD4+ T cells. Evidence for an implication of these $T$ cells in the pathogenesis of MS is mainly provided by studies, which reveal oligoclonal expansion of CD8+ and CD4+ T cells in CNS lesions and the CSF of MS patients. The robust oligoclonal expansion indicates that T cells locally react to CNS-intrinsic antigens (Hafler et al., 1988, Babbe et al., 2000, Jacobsen et al., 2002). Interestingly, identical CD8+ T cell clones can be detected in different lesions within one patient and memory CD8+ T cell clones persist in the CSF for several years (Babbe et al., 2000, Skulina et al., 2004).

Regulatory T cells appear also to be implicated in the pathogenesis of MS. In MS patients the suppressive function of regulatory $T$ cells is profoundly compromised, which possibly might contribute to the pathogenic activation of T helper 1 (Th1) and T helper 17 (Th17) cells (Viglietta et al., 2004, Haas et al., 2005).

Besides T cells, B cells have been appreciated to play a prominent role in the pathogenesis of MS. Local activity of plasma cells within the CNS leads to the appearance of immunoglobulins and oligoclonal bands in the CSF, which is an important diagnostic criterion for MS and is associated with disease activity (Walsh and Tourtellotte, 1986, Sharief and Thompson, 1991). As T cells, B cells clonally expand in the CSF of MS patients (Owens et al., 2003) and affinity maturation of B cells was detected within the CNS (von Budingen et al., 2012). The crucial role of 
B cells within the pathogenesis was revealed by clinical trials showing that treatment with the B cell-depleting antibody rituximab was beneficial for RRMS patients (Hauser et al., 2008).

The exact role of myelin-specific auto-reactive $T$ and $B$ cells is not fully understood. Increased numbers of high affinity myelin basic protein (MBP) specific Th1 and Th17 cells were reported in the peripheral blood of MS patients, but low numbers of these cells can also be detected in healthy donors (Olsson et al., 1992, Bielekova et al., 2004, Durelli et al., 2009). Furthermore, the oligoclonal $B$ cell response is not targeted to MBP, proteolipid protein (PLP) or myelin oligodendrocyte glycoprotein (MOG) and the antigen-specificity of the clonally expanded T and B cells has not been identified yet (Owens et al., 2009).

In addition to adaptive immune cells, innate immune cells are also implicated in the pathogenesis of MS. Most prominent examples are dendritic cells, macrophages and microglia. Dendritic cells isolated from MS patients display an activated phenotype and predominantly induce Th1 and Th17 differentiation (Karni et al., 2006, Vaknin-Dembinsky et al., 2006). Activated macrophages and microglia, which are abundant in active MS lesions, mainly display the proinflammatory M1 phenotype and contribute to inflammation through antigen-presentation and pro-inflammatory cytokine secretion (Gandhi et al., 2010, Vogel et al., 2013).

\subsubsection{Pathology}

MS patients typically present multifocal lesions within the CNS. These lesions can occur anywhere in the CNS, but are predominantly localized in characteristic predilection sites, including the optic nerve, periventricular and subcortical cerebral regions, the cerebellum, the brainstem and the spinal cord. Macroscopically, white matter lesions appear as rounded plaques with a brownish-gray color and compact consistency. The histopathological hallmarks of MS lesions are an inflammatory infiltrate, pronounced demyelination with variable efficiency of remyelination, axonal loss and reactive astrogliosis (Brück and Stadelmann, 2005).

Demyelinating white matter lesions can be classified according to the grade of inflammation (van der Valk and De Groot, 2000). Traditionally, three types of lesions are distinguished according to the density and the localization of the inflammatory infiltrate: active lesions, characterized by a pronounced inflammatory infiltrate, chronic active lesions, displaying a hypocellular center and an inflammatory rim and chronic inactive lesions, defined as hypocellular. Alternatively, the staging system proposed by Brück and Lassmann differentiates between early active, late active and inactive lesions, which are mainly distinguished by differential macrophage markers and myelin degradation products. Early active lesions are characterized by macrophages, which express the marker myeloid-related protein 14 (MRP14) and contain myelin degradation products positive for MBP, PLP, myelin-associated glycoprotein, MOG and cyclic nucleotide 
phosphodiesterase. In late active lesions macrophages only contain degradation products immunopositive for MBP and PLP. Inactive lesions still display varying numbers of macrophages, but these phagocytes do not contain any myelin proteins immunopositive for the markers mentioned above (Brück et al., 1995).

Active demyelinating lesions were shown to be heterogeneous regarding the mechanism of oligodendrocyte damage. Three different patterns of lesions can be typically observed in living MS patients. Pattern I and pattern II lesions are both characterized by a massive infiltration of $T$ cells and macrophages, but pattern II lesions additionally display considerable immunoglobulin and complement depositions. The typical feature of pattern III lesions is the pronounced apoptosis of oligodendrocytes in and around lesions. Interestingly, the lesion pattern may vary between different individuals, but within one patient it appears to remain stable over time (Lucchinetti et al., 2000).

Beside white matter lesions, gray matter demyelination occurs in supra- and infratentorial as well as spinal areas. The extent of gray matter demyelination was shown to exceed the one of white matter demyelination in most CNS areas (Gilmore et al., 2009). Cortical demyelination plays a central role in the pathogenesis of MS. Cortical lesions contain less inflammatory cells compared to white matter lesions, but display substantial axonal transection, neuronal apoptosis and synaptic loss (Peterson et al., 2001, Wegner et al., 2006). Imaging studies indicate that gray matter atrophy occurs early during the course of disease (De Stefano et al., 2003, Fisher et al., 2008).

One CNS-intrinsic repair mechanism is remyelination, an event that frequently occurs in the early phase of MS, but decreases during the chronic phase (Goldschmidt et al., 2009). The extent of remyelination varies greatly among individuals and remyelination often remains incomplete (Patrikios et al., 2006).

Chronic disability in MS is mainly associated with neuroaxonal degeneration - particularly in the corticospinal tracts. Axonal injury is an early event during the disease course, which correlates with the degree of inflammation (Trapp et al., 1998, Bitsch et al., 2000). The extent of axonal loss was described to be associated with disease duration and progression (Schirmer et al., 2011). Different mechanism are discussed which could contribute to neuroaxonal damage in MS, like direct damage of axons through reactive oxygen species and nitric oxide (Smith and Lassmann, 2002), Wallerian degeneration (Dziedzic et al., 2010), mitochondrial dysfunction (Dutta et al., 2006, Mahad et al., 2009, Campbell et al., 2011) and alterations in neurotransmission with increased excitotoxity (Pitt et al., 2003, Srinivasan et al., 2005, Dutta et al., 2006).

In addition to the well defined white and gray matter lesions, MS patients also display diffuse abnormalities in the normal-appearing white matter (NAWM) and also in the normalappearing gray matter. NAWM changes comprise low grade T cell infiltration (Kutzelnigg et al., 
2005, Androdias et al., 2010), microglia activation (Howell et al., 2010), the presence of microglial nodules (Singh et al., 2013), decreased axonal densities (Evangelou et al., 2000, Evangelou et al., 2000) and increased axonal damage (Kutzelnigg et al., 2005) as well as increased numbers of oligodendrocytes (Hoftberger et al., 2010). This diffuse damage of the NAWM is associated with white matter atrophy and disease progression (Evangelou et al., 2000, Evangelou et al., 2000, Kutzelnigg et al., 2005).

\subsubsection{Treatment}

Two paradigms have to be distinguished in the treatment of RRMS patients: First, the intervention therapy during an acute relapse and second, long term disease-modifying treatments.

The first line medication during an acute MS relapse is the intravenous application of high dose methylprednisolon. If glucocorticoid administration fails to control the exacerbation, plasma exchange is the second therapeutic option.

Today ten different medications are approved for the long term treatment of RRMS in Europe, which are classified into first line, second line and third line therapy according to their safety profile.

First line options are the different beta-interferons (IFN $\beta$ ), glatiramer acetate (GA) and the recently approved orally active substance dimethyl fumarate. All these drugs have a highly favorable safety profile and exert moderate disease modifying activities. Teriflunomide and alemtuzumab are also approved as first line medications for RRMS, but both drugs display more severe side effects in comparison to the before mentioned first line treatments.

Three different recombinant IFN $\beta$ compounds are available: Rebif ${ }^{\circledR}$ (Merck-Serono), Avonex ${ }^{\circledR}$ (Biogen-Idec) and Betaferon ${ }^{\circledast} /$ Extavia $^{\circledR}$ (Bayer-Schering/Novartis). All medications have to be applied by subcutaneous or intramuscular injections. IFN $\beta$ has proven to be beneficial in RRMS patients (Jacobs et al., 2000, Filippi et al., 2004, Bagnato et al., 2005, Kappos et al., 2007, Trojano et al., 2007) and its protective effects seem to be based on the reduction of antigen presentation, $\mathrm{T}$ cell expansion, $\mathrm{T}$ cell trafficking into the CNS and pro-inflammatory cytokine secretion (Yong, 2002).

GA (Copaxone ${ }^{\circledR}$, Teva Pharmaceutical Industries) is a synthetic peptide of four amino acids resembling MBP. As IFN $\beta$, GA is an injectable drug, which considerably attenuates RRMS (Johnson et al., 1995, Comi et al., 2001). The immunomodulatory effect of GA is thought to be due to the development of GA-specific suppressive T cells that mainly produce T helper 2 (Th2) cytokines (Aharoni et al., 1997, Neuhaus et al., 2000, Yong, 2002) and the increased secretion of brainderived neurotrophic factor (BDNF) by T cells (Ziemssen et al., 2002). 
Dimethyl fumarate (Tecfidera ${ }^{\circledR}$, Biogen-Idec) is an orally active compound approved for the treatment of psoriasis and, since 2013, RRMS. Dimethyl fumarate treatment was shown to be beneficial for RRMS patients (Fox et al., 2012, Gold et al., 2012), which is attributed to its neuroprotective antioxidative actions (Linker et al., 2011, Scannevin et al., 2012) and additional immunomodulatory properties (Ghoreschi et al., 2011).

Teriflunomide (Aubagio ${ }^{\circledR}$, Genzyme) and alemtuzumab (Lemtrada ${ }^{\circledR}$, Genzyme) were also approved as first line medication for RRMS in 2013. Both drugs showed beneficial effects in phase III studies (O'Connor et al., 2006, Coles et al., 2008, O'Connor et al., 2011, Cohen et al., 2012), but treatment was associated with undesirable side effects. Teriflunomide mediates its effects mainly by blocking the de novo synthesis of pyrimidines and subsequent inhibition of cell proliferation. This mechanism affects activated T cells, but also other proliferating cells, leading to undesired effects such as teratogenicity (Cherwinski et al., 1995, Greene et al., 1995, Warnke et al., 2009). Alemtuzumab is a humanized antibody against CD52 and administration of this antibody induces pronounced and long-lasting depletion of $\mathrm{T}$ and B cells (Klotz et al., 2012). Side effects of alemtuzumab are slightly increased rates of infection and the development of antibody-mediated autoimmunity, such as idiopathic thrombocytopenic purpura and thyroid disorders (Coles et al., 2008). Due to these adverse events, alemtuzumab is more likely to be chosen either as second line treatment or as first line therapy for patients with more aggressive MS in practice.

Second line treatments are applied when first line treatments fail to efficiently control disease activity. Therapeutic options are natalizumab and fingolimod, which are characterized by their higher therapeutic efficacy, but also slightly reduced tolerability.

Natalizumab (Tysabri ${ }^{\circledR}$, Biogen Idec) is a humanized monoclonal antibody that binds to the $\alpha 4$ subunit of $\alpha 4 \beta 1$ and $\alpha 4 \beta 7$ integrins and thereby prevents lymphocyte adhesion to endothelial cells of the BBB and CNS infiltration (Yednock et al., 1992). Natalizumab efficiently reduces disease activity in RRMS patients (Polman et al., 2006), but rarely patients develop progressive multifocal leukoencephalopathy (PML) upon treatment (Rudick et al., 2006). The incidence of PML appears to be strongly associated with John Cunningham virus (JCV) infection and therefore patients should be tested for anti-JCV antibodies prior to treatment with natalizumab (Derfuss and Kappos, 2013).

Fingolimod (Gilenya ${ }^{\circledR}$, Novartis) was the first orally active immunomodulator approved for the treatment of RRMS in 2011. Fingolimod is an unselective sphingosin-1-phosphat (S1P) receptor agonist, which exerts its beneficial effects in MS (Kappos et al., 2006, Kappos et al., 2010) mainly through the sequestration of lymphocytes within the lymph nodes (Brinkmann et al., 2002, Mandala et al., 2002). 
Mitoxantrone is an immunosuppressive drug approved as third line treatment for RRMS. It intercalates into the deoxyribonucleic acid (DNA) double helix and reduces $T$ and $B$ cell activation and proliferation and the activation of macrophages (Hartung et al., 2002). Mitoxantrone very efficiently reduces disease activity in RRMS (Edan et al., 2011), but due to its cardiotoxicity, bone marrow depression and the development of secondary malignancies it is only rarely applied if all other therapeutic options fail.

During the last ten years, several new drugs have been approved for the treatment of RRMS and additional promising substances like laquinimod, daclizumab and rituximab are currently under investigation to further ameliorate the therapy of RRMS patients.

\subsection{Experimental autoimmune encephalomyelitis}

\subsubsection{EAE induction}

Experimental autoimmune encephalomyelitis (EAE) is the most common animal model to investigate autoimmune aspects of MS. EAE was first described by Koritschoner and Schweinburg in 1925 who immunized rabbits with human spinal cord homogenate (Koritschoner and Schweinburg, 1925).

Typically EAE can be induced in susceptible animals by active immunization with distinct myelin peptides in complete Freund's adjuvant (CFA) and administration of pertussis toxin (Stromnes and Goverman, 2006). EAE studies are most frequently performed in mice, but also in rats and marmosets. The specific peptide, used to initiate EAE, depends on the mouse strain: C57BI/6 mice respond to $\mathrm{MOG}_{35-55}$ (Mendel et al., 1995), SJL/J mice to $\mathrm{PLP}_{139-151}$ (Tuohy et al., 1992), Biozzi $\mathrm{AB} / \mathrm{H}$ mice to $\mathrm{MOG}_{1-22}$ (Amor et al., 1994) and B10.PL mice to $\mathrm{MBP}_{1-9}$ (Ando et al., 1989). $\mathrm{MOG}_{35-55}$-induced $\mathrm{EAE}$ in $\mathrm{C} 57 \mathrm{BI} / 6$ mice is one of the most frequently used $\mathrm{EAE}$ models, since genetically modified mice are mainly available on the C57BI/6 background.

As alternative to active immunization, adoptive transfer of activated myelin-specific T cells is a suitable approach to passively induce EAE in rodents (Ben-Nun et al., 1981, Zamvil et al., 1986, Stromnes and Goverman, 2006).

The disease course of EAE is characterized by an ascending paralysis, which starts in the tail, typically ten to fourteen days after immunization, and subsequently affects the hind limbs, the abdominal muscles and the fore limbs. C57BI/6 mice display a monophasic disease course with variable remission leading to chronic neurological impairment. In contrast, SJL/J and Biozzi mice develop a relapsing-remitting form of EAE (Gold et al., 2006). 
During the last two decades the spectrum of EAE models was enriched by different genetically modified animals with spontaneous development of disease symptoms. Two examples are OSE and ODC-OVA/OT-1 mice. OSE (optico-spinal EAE) mice are double transgenic mice possessing MOG-specific $T$ cell and $B$ cell receptors. These animals are derived from crossbreedings of MOG-specific T cell receptor transgenic 2D2 (Bettelli et al., 2003) and MOGspecific B cell receptor transgenic Th mice (Litzenburger et al., 1998) and spontaneously develop optico-spinal EAE with an incidence of approximately 50\% (Krishnamoorthy et al., 2006). ODCOVA/OT-1 mice are double transgenic animals which express ovalbumin (OVA) specifically in oligodendrocytes (ODC) and contain CD8+ T cells carrying the OVA-specific T cell receptor OT-1. Up to $90 \%$ of these animals experience fulminant EAE starting 12 - 19 days after birth ( $N a$ et al., 2008).

\subsubsection{Effector cells in $\mathrm{MOG}_{35-55}$-induced $\mathrm{EAE}$}

The main effector cells in $\mathrm{MOG}_{35-55}$-induced $\mathrm{EAE}$ in $\mathrm{C} 57 \mathrm{BI} / 6$ mice are thought to be CD4+ $T$ cells. Encephalitogenic $M_{0 G} O_{35-55}$-specific $T$ cell clones were shown to express $V \beta 1, V \beta 6, V \beta 8$, $\mathrm{V} \beta 14$ and $\mathrm{V} \beta 15 \mathrm{~T}$ cell receptors (TCR), with V $\beta 8$ being the predominant form (40-43\%) (Mendel et al., 1995).

The first pathogenic effects of CD4+ T cells in EAE were described for IFNY-producing Th1 cells. Adoptive transfer of myelin-specific Th1 cells induces EAE (Baron et al., 1993), whereas genetical depletion of the lineage specific transcription factor T-bet renders animals resistant to EAE (Bettelli et al., 2004). IL-12 is a central cytokine for the differentiation of Th1 cells, but interestingly IL-12-deficient mice are still susceptible to EAE induction (Becher et al., 2002). In contrast, IL-23 was identified as crucial factor for autoimmune CNS inflammation (Cua et al., 2003).

The cytokine IL-23 promotes the development of IL-17-secreting CD4+ T cells (Th17 cells), which were subsequently recognized as the second central player for $\mathrm{T}$ cell-mediated autoimmunity in EAE. Th17 cells are highly encephalitogenic and promote EAE upon adoptive transfer (Langrish et al., 2005, Kroenke et al., 2008, Jäger et al., 2009).

Both T cell subpopulations - Th1 and Th17 cells - induce EAE with a comparable severity in adoptive transfer experiments (Kroenke et al., 2008, Jäger et al., 2009), but the pathological mechanisms differ, since Th1 cells mainly recruit macrophages to the CNS, whereas Th17 cells secrete chemoattractants for neutrophils (Kroenke et al., 2008).

Studies evaluating the impact of IFNY and IL-17 in the pathogenesis of EAE revealed that each of these cytokines alone is not essential. IL-17 neutralization and genetic depletion of IL-17 and its receptor attenuate disease severity, but IL-17-deficient as well as IFNY-deficient animals 
are still susceptible to EAE (Ferber et al., 1996, Hofstetter et al., 2005, Komiyama et al., 2006, Haak et al., 2009, Hu et al., 2010).

Anti-inflammatory regulatory $T$ cells represent the third important effector cell population in EAE. Regulatory T cells were shown to inhibit the activation of auto-reactive $T$ cells and the adoptive transfer of these IL-10 producing regulatory cells protects the recipient animals from EAE (Kohm et al., 2002, Zhang et al., 2004, Liu et al., 2006). During the course of disease, regulatory T cells accumulate in the CNS and they appear to be essential for the recovery phase (McGeachy et al., 2005).

The exact role of CD8+ T cells in EAE is still controversial. Several groups claim a regulatory effect of CD8+ T cells, since the depletion of $C D 8+T$ cells enhances EAE and the transfer of CD8+ CD28- T cells suppresses EAE (Najafian et al., 2003, Montero et al., 2004). In contrast, other studies provide evidence for a pathogenic role. Myelin-specific CD8+ T cells accumulate within the CNS (Ford and Evavold, 2005) and induce EAE upon adoptive transfer (Huseby et al., 2001, Sun et al., 2001). Furthermore, the activation of myelin-specific CD8+ T cells by viral infections can break T cell tolerance and induces CNS autoimmunity (Ji et al., 2010).

C57BI/6 mice fail to produce pathogenic antibodies upon immunization with $\mathrm{MOG}_{35-55}$ (Bourquin et al., 2003). Therefore, $\mathrm{MOG}_{35-55}$-induced $\mathrm{EAE}$ in $\mathrm{C} 57 \mathrm{BI} / 6$ mice is not a suitable approach to investigate the contribution of pathogenic demyelinating antibodies. Suitable models to study the role of pathogenic antibodies comprise peptide-induced EAE in $\mathrm{SJ} / \mathrm{J}$ or Biozzi $\mathrm{AB} / \mathrm{H}$ mice, MOG-induced EAE in dark Agouti and brown Norway rats (Krishnamoorthy and Wekerle, 2009) and MOG-specific B cell receptor transgenic (Th) mice (Litzenburger et al., 1998).

\subsubsection{The value of EAE for the development of MS therapies}

Preclinical studies, which evaluate the efficiency, safety and tolerability of a substance, are a prerequisite for the development of new therapeutic approaches. EAE has proven to be a valuable tool to investigate novel drugs for the treatment of MS. Four out of the ten currently approved MS medications were chosen to be evaluated in clinical trials based on their beneficial effects reported in different EAE models.

Glatiramer acetate (GA), a synthetic peptide of four amino acids, was developed in the 1970 's and its immunomodulatory properties were first investigated in different EAE models. In these studies GA completely inhibited acute EAE in guinea pigs, rabbits and rhesus monkeys (Teitelbaum et al., 1971, Teitelbaum et al., 1972, Teitelbaum et al., 1973, Teitelbaum et al., 1974), reduced the relapse rate in a relapsing-remitting model of EAE (Keith et al., 1979) and prevented adoptive transfer EAE (Aharoni et al., 1993). Besides its clinical efficiency, EAE studies revealed different components of the mechanism of action of GA, including the inhibition of antigen- 
dependent T cell activation and the induction of Th2-biased suppressor T cells (Teitelbaum et al., 1996, Aharoni et al., 1997).

Mitoxantrone is a DNA-intercalating immunosuppressive agent approved as third line medication for RRMS. First beneficial effects of the drug were observed in EAE studies, demonstrating complete inhibition of acute $E A E$, attenuation of relapsing-remitting $E A E$ and the reduction of established paralysis (Ridge et al., 1985, Levine and Saltzman, 1986, Lublin et al., 1987). Furthermore, data from EAE studies revealed that the potential mechanisms of action include the inhibition of lymphocyte proliferation as well as the reduced macrophage-mediated myelin destruction (Watson et al., 1991).

The humanized antibody natalizumab was developed on the basis of murine anti- $\alpha 4$ integrin antibodies, which efficiently prevented EAE induction by blocking the influx of activated T cells into the CNS of experimental animals (Yednock et al., 1992, Brocke et al., 1999). Notably, administration of these antibodies was beneficial during the whole course of disease and achieved remission of established clinical signs (Kent et al., 1995, Keszthelyi et al., 1996).

The first orally active compound approved for the treatment of RRMS was fingolimod. This unselective S1P receptor agonist was also first evaluated in EAE studies. Preventive treatment protected the animals from EAE induction and therapeutic treatment reduced EAE progression and relapse rates (Brinkmann et al., 2002, Kataoka et al., 2005). Furthermore, a dual mechanism of action was described in EAE studies. The immunomodulatory component is mediated by inhibiting the egress of lymphocytes from the lymph nodes, whereas CNS intrinsic effects appear to be triggered by the interaction with S1P1 receptors on the surface of astrocytes (Mandala et al., 2002, Choi et al., 2011).

Additional substances for the treatment of MS, which are currently under investigation in clinical trials, were previously evaluated in EAE models, like the quinoline-3-carboxamide laquinimod (Brunmark et al., 2002, Runström et al., 2006, Wegner et al., 2010, Aharoni et al., 2012, Mishra et al., 2012, Schulze-Topphoff et al., 2012, Thöne et al., 2012, Jolivel et al., 2013, Moore et al., 2013, Ruffini et al., 2013) and the humanized anti-CD25 antibody daclizumab (Hayosh and Swanborg, 1987, Engelhardt et al., 1989). 


\subsection{Natural killer cells}

\subsubsection{NK cell biology}

Natural killer (NK) cells originally received their name based on their natural ability to exert cytotoxic activity towards target cells without prior immunization. NK cells develop in the bone marrow from the same common lymphoid progenitor cell as T and B cells, but despite their classification as lymphocytes, NK cells recognize their targets using germline encoded receptors and are thus considered as component of the innate immune system.

The main effector functions of NK cells are the direct killing of target cells as well as the secretion of a variety of cytokines and chemokines. Thereby, NK cells considerably contribute to the defense against viral infections and play a major role in tumor surveillance (Cooper et al., 2001, Vivier et al., 2011).

NK cell effector functions need to be tightly controlled to assure efficient discrimination between self and foreign as well as healthy and diseased. The activation of NK cells is regulated by an array of activating and inhibitory receptors on their surface. Inhibitory receptors, such as killer cell immunoglobulin-like receptors $(\mathrm{KIR})$ and leukocyte immunoglobulin-like receptors in humans, Ly49 family members in mice and CD94/NKG2A in both species, mainly recognize MHC class I family molecules and contain immunoreceptor tyrosine-based inhibitory (ITIM) motifs within their intracellular domain. Recruitment and activation of $\mathrm{SH} 2$ domain-containing tyrosine phosphatases (SHP1 and SHP2) mediate inhibitory signaling (de Andrade et al., 2013). Activating NK cell receptors, like natural cytotoxicity receptors (NKp30, NKp44, NKp46, NKp80), NKG2D and CD244, bind to molecules associated with cellular stress and damage and trigger the activation of NK cells. NK cell activation is mediated by the recruitment of immunoreceptor tyrosine-based activation (ITAM) motif-containing molecules and the subsequent activation of downstream signaling molecules, such as nuclear factor kappa B (NF-KB), phospholipase $C_{\gamma}$ or protein kinase $C$. Another important receptor family binds to nectins and nectin-like proteins and includes the activating receptors DNAM-1 (DNAX Accessory Molecule-1) and TACTILE (T cell-activated increased late expression) as well as the inhibitory receptor TIGIT (T cell immunoreceptor with Ig and ITIM domains). Engagement of one single activating NK cell receptor is not sufficient to efficiently trigger NK cell activation, but a combination of different receptors is required to activate NK cells. One exception to this rule is CD16, which specifically recognizes the Fc region of immunoglobulins and mediates antibody dependent cellular cytotoxicity (ADCC) without bystander activation of any other receptor. Beside their regulation via activating and inhibitory receptors, NK cells do also react directly to different cytokines (Vivier et al., 2011, de Andrade et al., 2013, Long et al., 2013). 
During the last two decades the concept of NK cells as crucial regulatory element within immunity has emerged. On the one hand, NK cells support dendritic cell maturation and promote the Th1 response by secreting pro-inflammatory cytokines or by direct cell contact (Gerosa et al., 2002, Piccioli et al., 2002, Martin-Fontecha et al., 2004, Vitale et al., 2005). On the other hand, NK cells are able to kill immature dendritic cells in vitro (Wilson et al., 1999, Ferlazzo et al., 2002) and to inhibit T cell responses through the secretion of anti-inflammatory cytokines, p21-mediated cell cycle arrest and lysis of activated T cells (Cooper et al., 2001, Rabinovich et al., 2003, Trivedi et al., 2005, Cerboni et al., 2007, Lu et al., 2007, Deniz et al., 2008).

The overall pool of NK cells consists of different subpopulations. In humans, NK cells are classically subdivided into CD56 $6^{\text {dim }}$ CD16+ and CD56 bright CD16- NK cells. The CD56 dim subset is characterized by a low proliferation rate, high levels of inhibitory receptors and the expression of the adhesion molecules PEN-5 and LFA-1 and of the chemokine receptors CXCR1 and CX3CR1. In contrast, CD56 $6^{\text {bright }}$ NK cells have a high proliferative potential, display low levels of inhibitory receptors and express the adhesion molecule CD62L and the chemokine receptors CCR7 and CXCR3 (Cooper et al., 2001). Moreover, the two subsets differentially express the surface molecules CD27 and CD11b: CD11b+ NK cells are mainly found within the CD56 ${ }^{\operatorname{dim}}$ subpopulation, whereas CD27 expression is dominant on the CD56 $6^{\text {bright }}$ subset (Fu et al., 2011). Additionally, the two subsets differ with regard to their anatomical localization. CD56 ${ }^{\mathrm{dim}}$ NK cells are mainly found in the peripheral blood and the spleen, whereas CD56 bright $N K$ cells dominate in lymph nodes and tonsils (Vivier et al., 2008).

The two subsets also display functional differences. CD56 ${ }^{\operatorname{dim}}$ NK cells exhibit higher basal cytotoxicity and efficiently produce cytokines upon engagement of activating receptors, but are less responsive to cytokine stimulation. In contrast, lower basal cytotoxicity, but effective cytokine secretion in response to cytokine stimulation is ascribed to CD56 ${ }^{\text {bright }}$ NK cells (Cooper et al., 2001, Long et al., 2013).

The human NK cell marker CD56 is not expressed in the murine system. In C57BI/6 mice, NK cells are identified by the expression of the pan-NK cell markers NK1.1 and NKp46 on CD3negative cells and subpopulations can be classified according to the surface expression of CD27 and CD11b: CD27+ CD11b- NK cells, CD27+ CD11b+NK cells and CD27- CD11b+ NK cells (Chiossone et al., 2009). CD27+ CD11b- NK cells display a higher proliferative potential and express the chemokine receptor CXCR3. CD27- CD11b+ NK cells proliferate less vigorously and are characterized by high levels of inhibitory receptors (Kim et al., 2002, Hayakawa et al., 2006). All murine NK cell subsets are fully functional with regard to cytokine secretion and cytotoxicity, but in contrast to human NK cells, the predominant effector functions of individual subsets of murine NK cells are less well defined (Takeda et al., 2000, Kim et al., 2002, Hayakawa et al., 2006, Vahlne 
et al., 2008, Chiossone et al., 2009). Regarding their tissue distribution, CD27+ CD11b- NK cells are mainly present in the bone marrow and the lymph nodes, while CD27- CD11b+ NK cells are more abundant in the peripheral blood and the spleen (Vivier et al., 2008).

\subsubsection{NK cells and autoimmunity}

NK cells appear to be implicated in different autoimmune diseases like type I diabetes (TID), rheumatoid arthritis (RA), systemic lupus erythematosus (SLE) and MS, exerting either protective or destructive functions.

The role of NK cells in TID is still very controversial. On the one hand, NK cells only represent a minor fraction of the inflammatory infiltrate in the pancreas (Willcox et al., 2008). Reduced numbers and functional impairment of NK cells were observed in the peripheral blood of TID patients, suggesting a rather protective effect of NK cells (Rodacki et al., 2007). On the other hand, patients with coxsackie B4 enterovirus infected $\beta$ cells displayed considerable NK cell infiltration of the pancreas, leading to $\beta$ cell destruction (Dotta et al., 2007). Animal studies highlight these controversial effects by revealing protective (Lee et al., 2004, Lee et al., 2008) as well as destructive (Poirot et al., 2004) effects of NK cells.

Studies in RA patients point towards a dual role of NK cells. Activated CD56 bright NK cells were shown to specifically accumulate in the inflamed synovial tissue. These cells appear to contribute to the maintenance of Th1 inflammation and auto-reactive T cell activation through the production of pro-inflammatory cytokines (Pridgeon et al., 2003, de Matos et al., 2007, Zhang et al., 2007). In contrast to the inflamed joints, NK cell numbers and activity are considerably diminished in the blood of RA patients, pointing towards a regulatory role of these cells in the periphery (Aramaki et al., 2009, Park et al., 2009).

In SLE, NK cells seem to contribute to disease attenuation. Reduced NK cell numbers and impaired cytotoxicity are correlated with clinical manifestation of SLE and NK cells were reported to reduce the secretion of pathogenic auto-antibodies (Takeda and Dennert, 1993, Yabuhara et al., 1996, Park et al., 2009, Huang et al., 2011).

The contribution of NK cells to the pathogenesis of MS is not fully elucidated yet, but a large body of evidence supports the concept of a beneficial role of NK cells in MS. Several studies associate MS with reduced circulating NK cell numbers (Vranes et al., 1989, Munschauer et al., 1995, De Jager et al., 2008, Martinez-Rodriguez et al., 2010) and defective NK cell effector functions (Benczur et al., 1980, Hauser et al., 1981, Neighbour et al., 1982). Phases of particularly low NK cell activity precede disease exacerbation and the expansion of anti-inflammatory cytokine-secreting type II NK cells was observed during the remission phase (Takahashi et al., 
2001, Kastrukoff et al., 2003). The beneficial role of NK cells in MS is further supported by several studies reporting NK cell expansion and activation upon treatment with different MS medications.

\subsubsection{NK cells in the treatment of multiple sclerosis}

NK cell numbers and function were shown to be an important target of different MS therapies, underpinning the notion of a primarily regulatory and beneficial function of NK cells in MS.

MS patients treated with IFN $\beta$ display slightly decreased total numbers of circulating NK cells, but a robust expansion of the regulatory CD56 $6^{\text {bright }}$ subset in secondary lymphoid organs and the peripheral blood (Perini et al., 2000, Saraste et al., 2007, Vandenbark et al., 2009, MartinezRodriguez et al., 2010), which was significantly related to the therapy response (MartinezRodriguez et al., 2011).

Glatiramer acetate (GA) reduces auto-antigen presentation by different mechanisms. Besides its direct binding to major histocompatibility complex (MHC) molecules (Fridkis-Hareli et al., 1994), GA considerably enhances the cytotoxicity of NK cells towards immature dendritic cells. Furthermore, the cytokine secretion of NK cells is altered upon GA treatment, but total numbers remain unaffected (Al-Falahi et al., 2009, Sand et al., 2009).

Natalizumab treatment mainly interferes with the localization of NK cells, increasing circulating NK cells and reciprocally decreasing their numbers within the CNS, the lymph nodes and the spleen, suggesting that $\alpha 4$ integrin blockade alters the trafficking of NK cells (Putzki et al., 2010, Skarica et al., 2011, Gan et al., 2012, Planas et al., 2012).

Fingolimod treatment was reported to reduce the frequency of circulating CD56 ${ }^{\text {bright }}$ NK cells, without affecting their effector functions, which was attributed to an inhibition of NK cell egress from the lymph nodes, as evidenced for T and B cells (Mandala et al., 2002, Johnson et al., 2011). Interestingly, another study could show increased NK cell frequencies in the CSF of fingolimod-treated MS patients, potentially indicating a protective role of NK cells within the CNS compartment (Kowarik et al., 2011).

Most striking effects on NK cells were described for daclizumab, a humanized antibody recognizing the high affinity IL-2 receptor $\mathrm{CD} 25$, which is still under investigation for the treatment of MS. Daclizumab was originally designed to directly inhibit CD4+ T cell function, but in vivo studies only revealed a very mild effect on T cells. In contrast, daclizumab treatment induced a pronounced expansion of CD56 $6^{\text {bright }}$ NK cells, most likely mediated by the binding of IL-2 to the intermediate affinity receptor CD122, which is highly expressed on this subset (Bielekova et al., 2006, Wynn et al., 2010). This expansion was observed in the peripheral blood and the CSF of MS patients and was significantly correlated with reduced contrast-enhancing MRI lesions and a 
positive treatment response (Bielekova et al., 2006, Bielekova et al., 2011). Functionally, daclizumab-treated NK cells display increased proliferation rates and reduce the survival of activated T cells, which appears to be a central component of the mechanism of action in MS (Bielekova et al., 2006, Martin et al., 2010).

\subsection{The new immunomodulator laquinimod}

The new orally active immunomodulator laquinimod (LAQ) is currently under investigation for the treatment of MS. LAQ (5-chloro-N-ethyl-4-hydroxy-1-methyl-2-oxo-N-phenyl-1,2dihydroquinoline-3-carboxamide) (FIGURE 1 ) has been developed in a structure-activity relationship program through modification of its precursor substance roquinimex, which was withdrawn from clinical studies due to severe cardiopulmonary toxicities (Noseworthy et al., 2000, Jönsson et al., 2004). The substitution of the 5-H and the 12-N-methyl group of roquinimex by a $5-\mathrm{Cl}$ and a $12-\mathrm{N}$-ethyl group defined a new substance, LAQ, with increased efficacy and tolerability (Jönsson et al., 2004). Despite its higher efficiency to inhibit EAE compared to roquinimex (Brunmark et al., 2002, Jönsson et al., 2004), LAQ is not immunosuppressive and exerts clear immunomodulatory effects (Brunmark et al., 2002, Comi et al., 2008, Brück and Wegner, 2011).

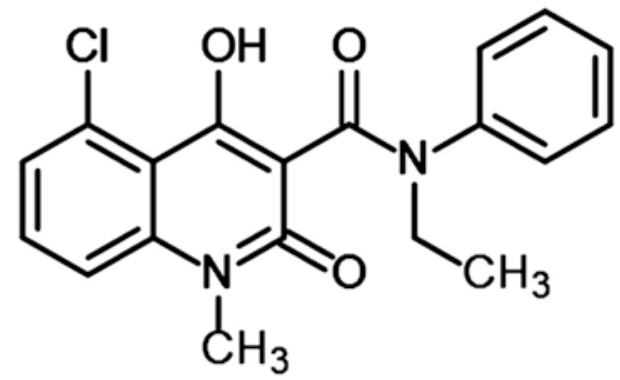

FIGURE 1: Structural formula of laquinimod

Structural formula of the new immunomodulatory substance laquinimod (5-chloro-N-ethyl-4-hydroxy-1methyl-2-oxo-N-phenyl-1,2-di-hydroquinoline-3carboxamide).

\subsubsection{Clinical studies}

Several clinical trials evaluated the safety, tolerability and therapeutic efficiency of LAQ in MS patients.

Phase II studies revealed a favorable safety profile and a significant reduction of gadolinium-enhancing (GdE) active MRI lesions, indicating decreased disease activity. (Polman et al., 2005, Comi et al., 2008, Comi et al., 2010). 
Subsequently, two randomized, double-blind, phase III studies, the ALLEGRO (Comi et al., 2012) and the BRAVO study (Vollmer et al., 2014) were performed and a third phase III trial, the CONCERTO study has recently been started. Data from the ALLEGRO trial indicated a moderate decrease of the annualized relapse rate by $23 \%$ in the LAQ group. Strikingly, LAQ exerted more dominant effects on the secondary endpoints "risk of disability progression" and "brain volume loss", which were markedly reduced by $30 \%$ and $43 \%$, respectively, indicating neuroprotective effects of LAQ treatment (Comi et al., 2012). Additional MRI measures further support the concept of a neuroprotective component within the mechanism of action of LAQ (Filippi et al., 2013).

Consequently, LAQ appears to have a dual mechanism of action, consisting of neuroprotective and immunomodulatory effects. The neuroprotective effect of LAQ could be partially due to an upregulation of brain-derived neurotrophic factor (BDNF) secretion in the periphery (Thöne et al., 2012). The immunomodulatory capacity of LAQ in MS patients was evidenced by considerably reduced frequencies of different dendritic cell populations in the peripheral blood after LAQ treatment and attenuated production of pro-inflammatory cytokines and chemokines by these dendritic cells upon stimulation ex vivo (Jolivel et al., 2013).

Taken together, data from clinical trials indicate that LAQ treatment is beneficial for MS patients, which is most likely mediated by immunomodulatory as well as neuroprotective effects. Still, the exact mechanism of action of LAQ in MS is not clear and a large panel of experimental studies was performed to elucidate different aspects of this mechanism.

\subsubsection{Experimental studies}

The effects of laquinimod treatment were studied in EAE. Preventive LAQ treatment efficiently inhibits acute EAE in mice (Brunmark et al., 2002, Aharoni et al., 2012, Mishra et al., 2012, Schulze-Topphoff et al., 2012, Thöne et al., 2012, Jolivel et al., 2013) and rats (Yang et al., 2004) in a dose-dependent manner (Yang et al., 2004, Runström et al., 2006, Wegner et al., 2010, Moore et al., 2013, Ruffini et al., 2013). Therapeutic treatment attenuates EAE severity (Wegner et al., 2010, Aharoni et al., 2012, Moore et al., 2013) and reduces the incidence of relapses in relapsing EAE models (Brunmark et al., 2002, Schulze-Topphoff et al., 2012, Jolivel et al., 2013).

Histological analysis of spinal cord sections from LAQ-treated EAE animals revealed decreased immune cell infiltration and microglia activation, which was associated with reduced demyelination, axonal damage and astrogliosis (Brunmark et al., 2002, Runström et al., 2006, Wegner et al., 2010, Mishra et al., 2012, Moore et al., 2013, Ruffini et al., 2013) and increased oligodendrocyte proliferation (Moore et al., 2013). 
Clinical trials point towards a dual role of $L A Q$ in the treatment of $M S$, exerting neuroprotective as well as immunomodulatory effects. This notion is underpinned by several experimental studies, which provide evidence for both components within its mechanisms of action

\subsubsection{Effects of LAQ within the CNS}

The first prerequisite for a substance to exert CNS intrinsic effects is its availability within the CNS. $13 \%$ of the blood concentration of LAQ reaches the CNS in rodents with EAE and 7 - 8\% in healthy animals (Brück and Wegner, 2011).

LAQ has shown to be neuroprotective in different experimental studies. Within the CNS, LAQ directly targets different cell types. LAQ inhibits toxin-mediated demyelination in the cuprizone-model and reduces the production of pro-inflammatory cytokines in astrocytes (Brück et al., 2012) and microglia (Mishra et al., 2013). Astrocytes show reduced NF-KB activity upon LAQ exposure (Brück et al., 2012), whereas in microglia mainly c-Jun N-terminal kinase (JNK) and protein kinase B (AKT) signaling are affected (Mishra et al., 2013). Furthermore, LAQ directly interferes with the neurotransmission in EAE animals, by reducing glutamatergic transmission and excitotoxicity as well as by enhancing GABAergic transmission (Ruffini et al., 2013) and callosal conduction (Moore et al., 2013). One further factor for the protective effect of LAQ in EAE seems to be BDNF, since the therapeutic efficiency of LAQ is reduced in BDNF-deficient animals and wildtype animals display increased BDNF mRNA levels in the spinal cord after LAQ treatment (Thöne et al., 2012).

\subsubsection{LAQ effects on immune cells}

Beside its CNS intrinsic effects, LAQ significantly modulates autoimmunity in EAE and recent studies indicate that antigen presenting cells are one of the direct targets of LAQ therapy.

LAQ treatment profoundly alters antigen presentation, regarding quantity and quality. On the one hand, LAQ reduces the frequency of antigen-presenting cells, monocytes as well as dendritic cells (Schulze-Topphoff et al., 2012, Thöne et al., 2012, Jolivel et al., 2013). On the other hand, LAQ changes the phenotype of antigen-presenting cells, by inducing a type II cytokine profile with decreased levels of IL-6, IL-12 and TNF and increased levels of IL-10 (Schulze-Topphoff et al., 2012) and by upregulating the co-stimulatory molecule CD86 which is associated with Th2 differentiation (Thöne et al., 2012, Jolivel et al., 2013). Adoptive transfer of LAQ-treated monocytes reduces EAE severity (Schulze-Topphoff et al., 2012) and this beneficial effect appears to be at least partially mediated by BDNF (Thöne et al., 2012). In vitro therapy approaches 
identified monocytes and dendritic cells as direct targets of LAQ and different pro-inflammatory signaling pathways, including NF-KB, signal transducer and activator of transcription (STAT) and JNK, were found to be altered by LAQ (Mishra et al., 2012, Schulze-Topphoff et al., 2012, Jolivel et al., 2013).

T cell differentiation is markedly biased towards anti-inflammatory subsets during LAQ therapy. LAQ considerably decreases the frequencies of pro-inflammatory Th1 and Th17 cells (Schulze-Topphoff et al., 2012, Jolivel et al., 2013) and their corresponding cytokines IFNy and IL17 (Wegner et al., 2010, Moore et al., 2013). In turn, anti-inflammatory regulatory T cells are increased by LAQ (Schulze-Topphoff et al., 2012, Jolivel et al., 2013). Moreover, LAQ slightly attenuates T cell proliferation in vivo (Jolivel et al., 2013) and inhibits the VLA-4-dependent adhesion of T cells to VCAM-1 upon CCL21 stimulation (Wegner et al., 2010).

The observed effects of LAQ on T cell differentiation are most likely indirect. Treatment with LAQ in vivo does not inhibit pro-inflammatory T cell differentiation in vitro (Schulze-Topphoff et al., 2012) and in vitro therapy of T cells does not influence their proliferation (Jolivel et al., 2013). The alterations in T cell differentiation are thought to be mediated by LAQ-treated antigen presenting cells, as LAQ-treated monocytes can inhibit T cell differentiation and proliferation as well as pro-inflammatory cytokine production in vitro (Schulze-Topphoff et al., 2012, Thöne et al., 2012, Jolivel et al., 2013).

In brief, according to the current literature, LAQ exerts its immunomodulatory effects mainly through the reduction and alteration of antigen presentation, which subsequently leads to a shift in T cell differentiation and attenuates EAE severity. Still, the mechanism of action of LAQ is not fully elucidated and different important cell populations like B cells and NK cells have not been studied so far in detail.

\subsection{Aims}

The overall aim of the present study is to further elucidate the immunomodulatory mechanism of action of LAQ by investigating effects of the substance on innate and adaptive immune cells in animals with EAE. In detail, this work intends to evaluate LAQ-mediated alterations in three different immune cell populations: $T$ cells, dendritic cells and most importantly NK cells.

\section{Effects of $L A Q$ on $T$ cells}

Pro-inflammatory T cell subsets are of central relevance for the pathogenesis of EAE and LAQ has been shown to efficiently inhibit EAE induction. Therefore, the present study aims to 
analyze effects of LAQ on pro-inflammatory Th1 and Th17 cells and anti-inflammatory regulatory $\mathrm{T}$ cells in vivo. The question, whether LAQ directly acts on T cells, will also be addressed by in vitro therapy experiments.

2. Effects of $L A Q$ on dendritic cells

Dendritic cells are potent antigen presenting cells and can profoundly influence the differentiation of T cells. Hence, this work aims to evaluate the effects of LAQ treatment on the frequencies of splenic dendritic cells and the expression of co-stimulatory molecules in vivo.

\section{Effects of LAQ on NK cells}

NK cells are involved in the pathogenesis of several autoimmune diseases and modulation of their phenotype and function appears to be associated with the beneficial effects of different MS treatments. NK cells may thus represent an interesting target for immunomodulatory therapies, however effects of LAQ treatment on NK cells have not been described before. Therefore, the central part of this project will assess the effects of LAQ on NK cell phenotype and function. Specific aims are to determine absolute NK cell numbers, NK cell frequency and NK cell subpopulations after LAQ administration. The impact of LAQ treatment on the activation state of NK cells will be assessed phenotypically, by analyzing surface activation markers and the NK cell receptor profile, and functionally by evaluating IFNY production, cytotoxic activity and the potential to reduce $\mathrm{T}$ cell proliferation in vitro. Furthermore, time course experiments will examine the kinetics of LAQ-mediated alterations in NK cells and direct effects of LAQ on NK cells will be evaluated using an in vitro therapy approach. Finally, this work will address the question whether NK cells are of central relevance for the mechanism of action of LAQ. For this purpose, NK cell-competent and NK cell-depleted heterozygous MOG-specific B cell receptor transgenic mice (Th/+) mice will be immunized with $M O G_{35-55}$ and treated with $L A Q$ or vehicle. The efficiency of LAQ treatment in the presence and absence of NK cells will be assessed using the clinical EAE score and survival rates.

The central aim of this study is to evaluate the effects of LAQ on NK cells and their importance within the mechanism of action of this new immunomodulatory drug. The results obtained in this work can lead to a better understanding of the mechanism of action of LAQ in EAE and might thereby contribute to optimize the future therapy of MS patients. 


\section{Materials and methods}

\section{$2.1 \quad$ Materials}

\subsubsection{Reagents}

TABLE 1: Reagents

\begin{tabular}{|c|c|}
\hline Reagent & Source of supply \\
\hline Acetic acid, $100 \%$ & Merck Millipore, Darmstadt, Germany \\
\hline Agarose & StarLab GmbH, Hamburg, Germany \\
\hline Annexin $V$ binding buffer, $10 x$ & eBioscience, San Diego, CA, USA \\
\hline BD FACS Shutdown Solution & BD Biosciences, Franklin Lakes, NJ, USA \\
\hline BD FACSClean ${ }^{\mathrm{TM}}$ & BD Biosciences, Franklin Lakes, NJ, USA \\
\hline BD FACSFlow $^{\mathrm{TM}}$ & BD Biosciences, Franklin Lakes, NJ, USA \\
\hline BD FACS ${ }^{\text {TM }}$ Lysing Solution, $10 x$ & BD Biosciences, Franklin Lakes, NJ, USA \\
\hline BD Pharm Lyse ${ }^{\mathrm{TM}}, 10 \mathrm{x}$ & BD Biosciences, Franklin Lakes, NJ, USA \\
\hline Boric acid & Merck Millipore, Darmstadt, Germany \\
\hline Crystal violet & Sigma Aldrich, St. Louis, MO, USA \\
\hline Cytofix/Cytoperm ${ }^{\mathrm{TM}}$ & BD Biosciences, Franklin Lakes, NJ, USA \\
\hline Cytofix ${ }^{\mathrm{TM}}$ & BD Biosciences, Franklin Lakes, NJ, USA \\
\hline DMSO (dimethyl sulfoxide) & Sigma Aldrich, St. Louis, MO, USA \\
\hline dNTP (desoxynucleoside triphosphate) mix & Thermo Scientific, Waltham, Massachusetts, USA \\
\hline $\begin{array}{l}\text { EDTA (ethylenediamine tetraacetic acid } \\
\text { disodiumsalt dihydrate) }\end{array}$ & Carl Roth, Karlsruhe, Germany \\
\hline Ethanol, $100 \%$ & Merck Millipore, Darmstadt, Germany \\
\hline Ethidium bromide & Sigma Aldrich, St. Louis, MO, USA \\
\hline FCS (fetal calf serum) & Sigma Aldrich, St. Louis, MO, USA \\
\hline FoxP3 Fixation/Permeabilization Concentrate & eBioscience, San Diego, CA, USA \\
\hline FoxP3 Fixation/Permeabilization Diluent & eBioscience, San Diego, CA, USA \\
\hline FoxP3 Permeabilization buffer, $10 x$ & eBioscience, San Diego, CA, USA \\
\hline GeneRuler ${ }^{\mathrm{TM}}, 100$ base pairs (bp) DNA ladder Plus & Thermo Scientific, Waltham, MA, USA \\
\hline Go-Taq $^{\circledR}$ DNA polymerase buffer, $5 x$ & Promega, Madison, WI, USA \\
\hline $\begin{array}{l}\text { HBSS (Hank's buffered salt solution) with } \mathrm{Ca}^{2+} \text { and } \\
\mathrm{Mg}^{2+}\end{array}$ & Sigma Aldrich, St. Louis, MO, USA \\
\hline $\mathrm{HCl}$ (hydrochloric acid) & Merck Millipore, Darmstadt, Germany \\
\hline $\begin{array}{l}\text { HEPES (4-(2-hydroxyethyl)-1- } \\
\text { piperazineethanesulfonic acid) buffer, } 1 \mathrm{M}\end{array}$ & Sigma Aldrich, St. Louis, MO, USA \\
\hline $\mathrm{HL}-1^{\mathrm{TM}}$ serum-free medium & Lonza, Basel, Switzerland \\
\hline
\end{tabular}




\begin{tabular}{|c|c|}
\hline Reagent & Source of supply \\
\hline Ionomycin & Sigma Aldrich, St. Louis, MO, USA \\
\hline Isopropyl alcohol & Merck Millipore, Darmstadt, Germany \\
\hline Ketamine, $10 \%$ & Medistar ${ }^{\circledR}$, Ascheberg, Germany \\
\hline LAQ (laquinimod) & Teva pharmaceutical industries Ltd., Netanya, Israel \\
\hline L-glutamine, $200 \mathrm{mM}$ & Sigma Aldrich, St. Louis, MO, USA \\
\hline LPS (lipopolysaccharide) & Sigma Aldrich, St. Louis, MO, USA \\
\hline Mannide monooleate & Sigma Aldrich, St. Louis, MO, USA \\
\hline $\begin{array}{l}\text { MEM (minimum essential medium) non-essential } \\
\text { amino-acids, } 100 \mathrm{x}\end{array}$ & Sigma Aldrich, St. Louis, MO, USA \\
\hline $\mathrm{NaCl}$ (sodium chloride) & Carl Roth, Karlsruhe, Germany \\
\hline $\mathrm{NaCl}$ (sodium chloride), $0.9 \%$ solution, sterile & B. Braun Melsungen AG, Germany \\
\hline Paraffin oil & Carl Roth, Karlsruhe, Germany \\
\hline PBS (phosphate buffered salt solution), sterile & Sigma Aldrich, St. Louis, MO, USA \\
\hline Penicillin, 10,000 units / streptomycin, $10 \mathrm{mg} / \mathrm{ml}$ & Sigma Aldrich, St. Louis, MO, USA \\
\hline Perm/Wash ${ }^{\text {TM }}$ buffer, $10 \mathrm{x}$ & BD Biosciences, Franklin Lakes, NJ, USA \\
\hline PMA (phorbol 12-myristate 13-acetate) & Sigma Aldrich, St. Louis, MO, USA \\
\hline RPMI-1640 (Roswell Park Memorial Institute-1640) & Sigma Aldrich, St. Louis, MO, USA \\
\hline SDS (sodium dodecyl sulfate) & Sigma Aldrich, St. Louis, MO, USA \\
\hline Sodium pyruvate, $100 \mathrm{mM}$ & Sigma Aldrich, St. Louis, MO, USA \\
\hline $\begin{array}{l}\text { TMB }\left(3,3^{\prime}, 5,5^{\prime} \text {-tetramethylbenzidine) substrate }\right. \\
\text { solution }\end{array}$ & eBioscience, San Diego, CA, USA \\
\hline Tris (tris(hydroxymethyl)aminomethane) & Carl Roth, Karlsruhe, Germany \\
\hline Trypan blue & Sigma Aldrich, St. Louis, MO, USA \\
\hline Xylazine solution, $20 \mathrm{mg} / \mathrm{ml}$ & Ecuphar, Oostkamp, Belgium \\
\hline$\beta$-mercaptoethanol & Sigma Aldrich, St. Louis, MO, USA \\
\hline
\end{tabular}

\section{Abbreviations}

$\mathrm{Ca}^{2+}$ : calcium ion; DNA: deoxyribonucleic acid; FACS: fluorescence-activated cell sorting; FoxP3: forkhead box P3; MACS: magnetic-activated cell sorting; $\mathbf{M g}^{2+}$ : magnesium ion

\subsubsection{Solutions, buffers and cell culture media}

TABLE 2: Solutions, buffers and cell culture media

\begin{tabular}{ll}
\hline Solution & Composition \\
\hline Acetic acid, 33\% & Bidistilled water \\
& $33 \%$ acetic acid, $100 \%$
\end{tabular}




\begin{tabular}{|c|c|}
\hline Solution & Composition \\
\hline \multirow[t]{3}{*}{ CFA (Complete Freund's Adjuvant) } & Paraffin oil \\
\hline & $15 \%$ mannide monooleate \\
\hline & $6.7 \mathrm{mg} / \mathrm{ml}$ Mycobacterium tuberculosis H37RA \\
\hline \multirow[t]{2}{*}{ CFSE (carboxyfluorescein succinimidyl ester) buffer } & PBS, sterile \\
\hline & $0.1 \% \mathrm{BSA}$ \\
\hline \multirow[t]{3}{*}{ Crystal violet staining solution } & Bidistilled water \\
\hline & $0.4 \%$ crystal violet \\
\hline & $8 \%$ ethanol, $100 \%$ \\
\hline \multirow[t]{2}{*}{ FACS (fluorescence-activated cell sorting) buffer } & PBS, sterile \\
\hline & $2 \%$ FCS \\
\hline \multirow[t]{3}{*}{ Ketamine/Xylazine anesthesia } & $\mathrm{NaCl}, 0.9 \%$, sterile \\
\hline & $12 \%$ ketamine \\
\hline & $10 \%$ xylazine \\
\hline \multirow[t]{4}{*}{ MACS (magnetic-activated cell sorting) buffer } & PBS, sterile \\
\hline & $0.05 \% \mathrm{BSA}$ \\
\hline & $2 \mathrm{mM}$ EDTA \\
\hline & $\mathrm{pH} 7.2$ \\
\hline \multirow[t]{9}{*}{$\mathrm{RPMI}_{\text {complete }}$} & RPMI-1640 \\
\hline & $10 \%$ FCS \\
\hline & $1 \mathrm{mM}$ sodium pyruvate \\
\hline & $1 \mathrm{x}$ non-essential amino acids \\
\hline & 13 mM HEPES buffer \\
\hline & $50 \mu \mathrm{M} \beta$-mercaptoethanol \\
\hline & 100 units penicillin \\
\hline & $0.1 \mathrm{mg} / \mathrm{ml}$ streptomycin \\
\hline & $2 \mathrm{mM}$ L-glutamine \\
\hline
\end{tabular}




\begin{tabular}{ll}
\hline Solution & Composition \\
\hline Tail lysis buffer & Bidistilled water \\
& $0.1 \mathrm{M}$ Tris- $\mathrm{HCl} \mathrm{pH} 8.5$ \\
& $5 \mathrm{mM}$ EDTA \\
& $200 \mathrm{mM} \mathrm{NaCl}$ \\
& $0.2 \% \mathrm{SDS}$ \\
& pH 8.5 \\
TBE (Tris/borate/EDTA) buffer & Bidistilled water \\
$90 \mathrm{mM}$ Tris \\
$90 \mathrm{mM}$ boric acid \\
2 mM EDTA \\
\hline
\end{tabular}

\section{Abbreviations}

BSA: bovine serum albumin; EDTA: ethylenediamine tetraacetic acid disodiumsalt dehydrate; FCS: fetal calf serum; $\mathrm{HCl}$ : hydrochloric acid; HEPES: 4-(2-hydroxyethyl)-1-piperazineethanesulfonic acid; $\mathbf{N a C l}$ : sodium chloride; PBS: phosphate buffered salt solution; RPMI-1640: Roswell Park Memorial Institute1640 medium; SDS: sodium dodecyl sulfate; Tris: tris(hydroxymethyl)aminomethane

\subsubsection{Cell lines and bacteria}

TABLE 3: Cell lines and bacteria

\begin{tabular}{ll}
\hline Organism & Source of supply \\
\hline B16F10 melanoma cell line & Kindly provided by Prof. Evelyn Ullrich, Children's \\
& Hospital, Department of Pediatric Hematology and \\
& Oncology, Goethe-University, Frankfurt, Germany \\
& \\
Mycobacterium tuberculosis H37RA, non-viable & DIFCO, Detroit, MI, USA
\end{tabular}

\subsubsection{Proteins, cytokines and dyes}

TABLE 4: Proteins, enzymes and inhibitors

\begin{tabular}{ll}
\hline Proteins, enzymes, inhibitors & Source of supply \\
\hline BSA (bovine serum albumin) & SERVA Electrophoresis GmbH, Heidelberg, Germany \\
MOG $_{35-55}$ & Institute of Medical Immunology, University Medical \\
& Center Charité, Berlin, Germany \\
PTX (pertussis toxin) & List biological laboratories, Campbell, CA, USA \\
Collagenase D & Roche, Basel, Switzerland
\end{tabular}




\begin{tabular}{ll}
\hline Proteins, enzymes, inhibitors & Source of supply \\
\hline DNase I & Roche, Basel, Switzerland \\
Go-Taq ${ }^{\oplus}$ DNA polymerase & Promega, Madison, WI, USA \\
Proteinase K & Sigma Aldrich, St. Louis, MO, USA \\
Trypsin, 0.05\% & Gibco/Invitrogen, Carlsbad, CA, USA \\
GolgiStop & BD Biosciences, Franklin Lakes, NJ, USA \\
\hline
\end{tabular}

\section{Abbreviations}

DNA: deoxyribonucleic acid; $\mathbf{M O G}_{35-55}$ : myelin oligodendrocyte glycoproteine amino acids $35-55$

TABLE 5: Monoclonal antibodies for flow cytometry

\begin{tabular}{|c|c|c|c|c|}
\hline Specificity & Fluorochrome & Clone & Dilution & Source of supply \\
\hline B220 & PerCP & RA3-6B2 & $1: 200$ & $\mathrm{BD}$ \\
\hline CD11b & APC & $\mathrm{M} 1 / 70$ & $1: 200$ & $\mathrm{eB}$ \\
\hline $\mathrm{CD} 11 \mathrm{~b}$ & PE-Cy7 & $\mathrm{M} 1 / 70$ & $1: 200$ & eB \\
\hline CD11b & PerCP & M1/70 & $1: 200$ & $\mathrm{BL}$ \\
\hline CD11c & PE & N418 & $1: 200$ & $B L$ \\
\hline CD16/CD32 & - & 93 & $1: 100$ & $\mathrm{BL}$ \\
\hline CD19 & APC-Cy7 & $6 \mathrm{D} 5$ & $1: 200$ & $\mathrm{BL}$ \\
\hline CD19 & FITC & eBio1D3 & $1: 200$ & $\mathrm{eB}$ \\
\hline $\mathrm{CD} 2$ & FITC & RM2-5 & $1: 200$ & $\mathrm{eB}$ \\
\hline CD205 & PE-Cy7 & NLDC-145 & $1: 200$ & $\mathrm{BL}$ \\
\hline CD244.2 & FITC & $\mathrm{m} 2 \mathrm{~B} 4$ & $1: 200$ & $\mathrm{BL}$ \\
\hline $\mathrm{CD} 25$ & APC & PC61.5 & $1: 200$ & $\mathrm{eB}$ \\
\hline $\mathrm{CD} 27$ & PE & LG.3A10 & $1: 200$ & $\mathrm{BL}$ \\
\hline CD3 & FITC & $145-2 C 11$ & $1: 200$ & $\mathrm{BL}$ \\
\hline CD3 & PE-Cy7 & $145-2 C 11$ & $1: 200$ & $\mathrm{eB}$ \\
\hline CD3 & APC-Cy7 & $145-2 C 11$ & $1: 200$ & $\mathrm{BL}$ \\
\hline CD4 & PerCP & RM4-5 & $1: 200$ & $\mathrm{BL}$ \\
\hline CD44 & APC & IM7 & $1: 200$ & $\mathrm{eB}$ \\
\hline CD45 & APC-Cy & 30-F11 & $1: 200$ & $\mathrm{BL}$ \\
\hline CD62L & PE & MEL-14 & $1: 200$ & $\mathrm{eB}$ \\
\hline CD69 & PE & $\mathrm{H} 1.2 \mathrm{~F} 3$ & $1: 200$ & $\mathrm{eB}$ \\
\hline CD69 & PE-Cy7 & $\mathrm{H} 1.2 \mathrm{~F} 3$ & $1: 200$ & $\mathrm{BL}$ \\
\hline $\mathrm{CD} 8$ & PE-Cy7 & $53-6.7$ & $1: 200$ & $e B$ \\
\hline CD8 & APC-Cy7 & 53-6.7 & $1: 200$ & $B L$ \\
\hline CD80 & APC & 16-10A1 & $1: 200$ & $\mathrm{BL}$ \\
\hline
\end{tabular}




\begin{tabular}{|c|c|c|c|c|}
\hline Specificity & Fluorochrome & Clone & Dilution & Source of supply \\
\hline CD86 & APC-Cy7 & GL1 & $1: 200$ & $\mathrm{BL}$ \\
\hline CXCR3 & PerCP/Cy5.5 & CXCR3-173 & $1: 200$ & $B L$ \\
\hline DNAM-1 & PE & $10 \mathrm{E} 5$ & $1: 200$ & $\mathrm{BL}$ \\
\hline FoxP3 & PE & FJK-16S & $1: 100$ & $\mathrm{eB}$ \\
\hline IFNY & PE & XMG1.2 & $1: 200$ & eB \\
\hline IFNY & APC & XMG1.2 & $1: 200$ & eB \\
\hline IL-17A & PE-Cy7 & eBio17B7 & $1: 200$ & $\mathrm{eB}$ \\
\hline LFA-1 & PE & $\mathrm{M} 17 / 4$ & $1: 200$ & eB \\
\hline Ly49A & PE & A1 & $1: 200$ & eB \\
\hline Ly49C/I/F/H & FITC & 14B11 & $1: 200$ & $\mathrm{eB}$ \\
\hline Ly49D & PE & $4 \mathrm{E} 5$ & $1: 200$ & $B L$ \\
\hline Ly49G2 & FITC & eBio4D11 & $1: 200$ & eB \\
\hline $\mathrm{MHCll}$ & FITC & M5/114.15.2 & $1: 200$ & $B L$ \\
\hline NK1.1 & PE-Cy7 & PK-136 & $1: 200$ & $B L$ \\
\hline NK1.1 & APC & PK-136 & $1: 200$ & $\mathrm{BL}$ \\
\hline NKG2D & APC & CX5 & $1: 200$ & $B L$ \\
\hline NKp46 & PerCP & $29 \mathrm{~A} 1.4$ & $1: 200$ & $B L$ \\
\hline NKp46 & PE & 29A1.4 & $1: 200$ & $B L$ \\
\hline TACTILE & PE & 3.3 & $1: 200$ & $B L$ \\
\hline TIGIT & PE & $1 \mathrm{Gg}$ & $1: 200$ & $\mathrm{BL}$ \\
\hline$\gamma \delta$ TCR & APC & eBioGL3 & $1: 200$ & eB \\
\hline
\end{tabular}

\section{Abbreviations}

APC: allophycocyanin; BD: BD Biosciences, Franklin Lakes, NJ, USA; BL: BioLegend, San Diego, CA, USA; eB: eBioscience, San Diego, CA, USA; CD: cluster of differentiation; CXCR3: chemokine (C-X-C motif) receptor 3; Cy7: cyanine 7; DNAM-1: DNAX accessory molecule-1; FITC: fluorescein isothiocyanate; FoxP3: forkhead box P3; IFNY: interferon gamma; IL-17A: interleukin 17A; LFA-1: leukocyte function-associated antigen-1; MHCII: major histocompatibility complex class II molecule; NK: natural killer; NKG2D: natural killer group 2, member D; NKp46: natural killer cell p46-related protein; PE: phycoerythrin; PerCP: peridinin chlorophyll protein; TACTILE: T cell-activated increased late expression; TCR: T cell receptor; TIGIT: T cell immunoreceptor with Ig and ITIM domains 
TABLE 6: Monoclonal antibodies for antigen-independent activation of $\mathrm{T}$ cell proliferation in vitro

\begin{tabular}{lll}
\hline Specificity & Clone & Source of supply \\
\hline CD3 & $145-2 C 11$ & Bio X Cell, West Lebanon, NH, USA \\
CD28 & PV-1 & Bio X Cell, West Lebanon, NH, USA \\
\hline
\end{tabular}

\section{Abbreviations}

CD: cluster of differentiation

TABLE 7: Monoclonal antibodies for NK cell depletion in vivo

\begin{tabular}{llll}
\hline Specificity & Clone & Application & Source of supply \\
\hline NK1.1 & PK-136 & In vivo depletion of NK cells & Bio X Cell, West Lebanon, NH, USA \\
unknown & C1.18.4 & Isotype control antibody & Bio X Cell, West Lebanon, NH, USA \\
\hline
\end{tabular}

\section{Abbreviations}

NK: natural killer

TABLE 8: Cytokines

\begin{tabular}{ll}
\hline Cytokine & Source of supply \\
\hline GM-CSF, mouse, recombinant & PeproTech, Rocky Hill, NJ, USA \\
IL-12, mouse, recombinant & PeproTech, Rocky Hill, NJ, USA \\
IL-18, mouse, recombinant & PeproTech, Rocky Hill, NJ, USA \\
IL-2, human, recombinant & Novartis, Nuremberg, Germany \\
\hline
\end{tabular}

\section{Abbreviations}

GM-CSF: granulocyte macrophage colony-stimulating factor; IL: interleukin

TABLE 9: Fluorescent dyes

\begin{tabular}{llll}
\hline Name & Fluorochrome & Dilution & Source of supply \\
\hline Annexin V & APC & $1: 20$ & eBioscience, San Diego, CA, USA \\
$7-A A D$ & - & $1: 40$ & eBioscience, San Diego, CA, USA \\
\hline
\end{tabular}

\section{Abbreviations}

7-AAD: 7-aminoactinomycin D; APC: allophycocyanin 


\subsubsection{Applied kits}

TABLE 10: Kits

\begin{tabular}{ll}
\hline Kit & Source of supply \\
\hline CD11c MicroBeads & Miltenyi Biotec, Bergisch Gladbach, Germany \\
CD4+ T cell isolation kit II, mouse & Miltenyi Biotec, Bergisch Gladbach, Germany \\
CellTrace ${ }^{\text {TM }}$ CFSE Cell Proliferation Kit & Life Technologies, Carlsbad, CA, USA \\
Mouse IFNy DuoSet ${ }^{\circledR}$ ELISA development system & R\&D Systems, Minneapolis, MN, USA \\
Mouse IL-17 DuoSet ${ }^{\circledR}$ ELISA development system & R\&D Systems, Minneapolis, MN, USA \\
NK cell isolation kit II, mouse & Miltenyi Biotec, Bergisch Gladbach, Germany \\
\hline
\end{tabular}

\section{Abbreviations}

CD: cluster of differentiation; CFSE: carboxyfluorescein succinimidyl ester; ELISA: enzyme linked immunosorbent assay; IFNY: interferon gamma; IL-17: interleukin 17; NK: natural killer

\subsubsection{Oligonucleotide primers}

TABLE 11: Oligonucleotide primers

\begin{tabular}{|c|c|c|c|}
\hline Name & Sequence & Function & Used for genotyping of \\
\hline olMR1746 & $\begin{array}{l}\text { 5'-GAG GTT CCG CTA CGA CTC } \\
\text { TG-3' }\end{array}$ & $\begin{array}{l}\text { Forward primer for the } \\
\text { mutant allele }\end{array}$ & Rag1 $^{-1-}$ mice \\
\hline olMR8162 & $\begin{array}{l}\text { 5'-TGG ATG TGG AAT GTG TGC } \\
\text { GAG-3' }\end{array}$ & $\begin{array}{l}\text { Forward primer for the } \\
\text { wild type allele }\end{array}$ & Rag1 $^{-/-}$mice \\
\hline olMR3104 & $\begin{array}{l}\text { 5'-CCG GAC AAG TTT TTC ATC } \\
\text { GT-3' }\end{array}$ & $\begin{array}{l}\text { Common reverse } \\
\text { primer }\end{array}$ & Rag1 $1^{-/-}$mice \\
\hline olMR6711 & $\begin{array}{l}\text { 5'-CCC GGG CAA GGC TCA GCC } \\
\text { ATG CTC CTG-3' }\end{array}$ & $\begin{array}{l}\text { Forward primer for the } \\
\text { transgene }\end{array}$ & 2D2 mice \\
\hline olMR6712 & $\begin{array}{l}\text { 5'-GCG GCC GCA ATT CCC AGA } \\
\text { GAC ATC CCT CC-3' }\end{array}$ & $\begin{array}{l}\text { Reverse primer for the } \\
\text { transgene }\end{array}$ & 2D2 mice \\
\hline 8.18C5-sense & $\begin{array}{l}\text { 5'-TGA GGA CTC TGC CGT CTA } \\
\text { TTA CTG T- } 3^{\prime}\end{array}$ & $\begin{array}{l}\text { Forward primer for the } \\
\text { transgene }\end{array}$ & Th mice \\
\hline 8.18C5-antisense & $\begin{array}{l}\text { 5'-GGA GAC TGT GAG AGT GGT } \\
\text { GCC T-3' }\end{array}$ & $\begin{array}{l}\text { Reverse primer for the } \\
\text { transgene }\end{array}$ & Th mice \\
\hline
\end{tabular}




\begin{tabular}{llll}
\hline Name & Sequence & Function & Used for genotyping of \\
\hline mIgH-sense & 5'-ATT GGT CCC TGA CTC AAG $^{\prime}$ & Forward primer for the & Th mice \\
& AGA TG-3' & wild type allele & \\
mlgH-antisense & $5^{\prime}-$ TGG TGC TCC GCT TAG TCA & Reverse primer for the & Th mice \\
& AA-3' & wild type allele & \\
\hline
\end{tabular}

\section{Source of supply}

All oligonucleotide primers were purchased from Eurofins MWG, Ebersberg, Germany

\section{Abbreviations}

2D2 mice: MOG-specific T cell receptor transgenic mice; mlgH: murine immunoglobulin heavy chain; $\operatorname{Rag1}^{-1-}$ : Recombination activating gene 1 deficient mice; Th mice: MOG-specific B cell receptor transgenic mice;

\subsubsection{Consumables}

TABLE 12: Consumables

\begin{tabular}{|c|c|}
\hline Consumable & Source of supply \\
\hline Bottle top filter, $0.2 \mu \mathrm{m}$ & Sarstedt, Nuembrecht, Germany \\
\hline Cell culture dish, $60 \times 15 \mathrm{~mm}$ & Greiner bio-one, Kremsmuenster, Austria \\
\hline Cell culture flask, $75 \mathrm{~cm}^{2}$ & Greiner bio-one, Kremsmuenster, Austria \\
\hline Cell culture plates, flat bottom ( 6 well, 24 well, 96 well) & Greiner bio-one, Kremsmuenster, Austria \\
\hline Cell strainer $(70 \mu \mathrm{m}, 40 \mu \mathrm{m})$ & BD Biosciences, Franklin Lakes, NJ, USA \\
\hline Cryogenic tubes & Thermo Scientific, Waltham, MA, USA \\
\hline FACS tube, $5 \mathrm{ml}$ & BD Biosciences, Franklin Lakes, NJ, USA \\
\hline Filter units, $0.2 \mu \mathrm{m}$ & GE Healthcare, Chalfont St Giles, Great Britain \\
\hline GentleMACS ${ }^{\mathrm{M}}$ C-tube & Miltenyi Biotec, Bergisch Gladbach, Germany \\
\hline HTS Transwell ${ }^{\circledR}-96$ Well Permeable Support & Corning, Corning, NY, USA \\
\hline LS columns & Miltenyi Biotec, Bergisch Gladbach, Germany \\
\hline Needles & BD Biosciences, Franklin Lakes, NJ, USA \\
\hline Nunc $^{\mathrm{TM}}$ MaxiSorp ${ }^{\circledR} 96$ well ELISA plate & Thermo Scientific, Waltham, MA, USA \\
\hline Nunc $^{\mathrm{TM}}$ MicroWell ${ }^{\mathrm{TM}}$ Plates, 96 well round bottom & Thermo Scientific, Waltham, MA, USA \\
\hline Pre-separation filters, $30 \mu \mathrm{m}$ & Miltenyi Biotec, Bergisch Gladbach, Germany \\
\hline Syringes & BD Biosciences, Franklin Lakes, NJ, USA \\
\hline Tubes (50 ml, $15 \mathrm{ml}, 2 \mathrm{ml}, 1,5 \mathrm{ml}, 0.2 \mathrm{ml}$ ) & Sarstedt, Nuembrecht, Germany \\
\hline
\end{tabular}

\section{Abbreviations}

ELISA: enzyme linked immunosorbent assay; FACS: fluorescence-activated cell sorting; MACS: magneticactivated cell sorting; 


\subsubsection{Technical devices}

TABLE 13: Technical devices

\begin{tabular}{|c|c|}
\hline Device & Source of supply \\
\hline Centrifuge $5415 \mathrm{R}$ & Eppendorf, Hamburg, Germany \\
\hline Centrifuge $5810 \mathrm{R}$ & Eppendorf, Hamburg, Germany \\
\hline FACS Aria $^{\mathrm{TM}}$ II & BD Biosciences, Franklin Lakes, NJ, USA \\
\hline FACS Canto $^{\mathrm{TM}}$ II & BD Biosciences, Franklin Lakes, NJ, USA \\
\hline gentleMACS ${ }^{\mathrm{TM}}$ Dissociator & Miltenyi Biotec, Bergisch Gladbach, Germany \\
\hline Hera cell 150 incubator & Heraeus, Hanau, Germany \\
\hline iMark $^{\mathrm{TM}}$ microplate reader & Bio-Rad, Munich, Germany \\
\hline Neubauer chamber & Superior Marienfeld, Lauda-Koenigshofen, Germany \\
\hline Power supply & Bio-Rad, Munich, Germany \\
\hline QuadroMACS ${ }^{\mathrm{TM}}$ separator & Miltenyi Biotec, Bergisch Gladbach, Germany \\
\hline Speed vacuum Concentrator 5301 & Eppendorf, Hamburg, Germany \\
\hline T3 Thermocycler & Biometra, Germany \\
\hline Thermo mixer comfort & Eppendorf, Hamburg, Germany \\
\hline UV transluminator & Vilber Lourmat, Eberhardzell, Germany \\
\hline Nalgene $^{\mathrm{TM}}$ Cryo 1 C Freezing Container & Thermo Scientific, Waltham, MA, USA \\
\hline
\end{tabular}

\section{Abbreviations}

FACS: fluorescence-activated cell sorting; MACS: magnetic-activated cell sorting;

\subsubsection{Software}

TABLE 14: Software

\begin{tabular}{lll}
\hline Software & Application & Source of supply \\
\hline BD FACSDiva Software 6.1.2 & Data acquisition flow cytometry & BD Biosciences, Franklin Lakes, NJ, \\
& & USA \\
FlowJo 7.6.1 & Data analysis flow cytometry & Tree Star Inc., Ashland, OR, USA \\
GraphPad Prism 5.01 & Statistical analysis & GraphPad software Inc., La Jolla, \\
& Graphs & CA, USA \\
PSRemote 1.6.5 & Gel documentation & Breeze systems limited, \\
& & Camberley, UK \\
SAS ${ }^{\circledR} 9.3$ & Statistical analysis & SAS Institute Inc., Cary, NC, USA \\
\hline
\end{tabular}




\subsection{Animals}

\subsubsection{Mouse strains}

The following mouse strains were used for in vivo experiments and/or cell isolations:

\section{C57BI/6J mice}

Seven- to eight-weeks-old female C57BI/6J mice were purchased from Charles River laboratories, Sulzfeld, Germany.

\section{Rag1-deficient mice}

Rag1-deficient $\left(\operatorname{Rag}^{-1}\right)$ mice were developed and characterized by Mombaerts and colleagues in 1992 and subsequently backcrossed to C57BL/6J for more than 10 generations. They are characterized by a complete lack of mature B and T cells and are a valuable tool to investigate $B$ and T cell-dependant and independent effects on the immune system (Mombaerts et al., 1992).

$2 D 2$ mice

2D2 mice were generated and characterized by Bettelli and colleagues in 2003. $90-95 \%$ of the CD4+ $T$ cells in 2D2 animals express the $M_{30 G}{ }_{35-55}$-specific $T$ cell receptor V $\alpha 3.2 / \mathrm{V} \beta 11$ (Bettelli et al., 2003). A small percentage of 2D2 mice develops spontaneous EAE (2-5\%) and around 40\% of the 2D2 mice develop a spontaneous isolated neuritis of the optic nerves within 1 year. 2D2 CD4+ T cells were used as reporter cells in a number of assays.

Th mice

Th mice, generated and characterized by Litzenburger and colleagues, are IgH chain knock in mice in which the VDJ region of the hybridoma 8.18-C5 is inserted into the regular location of the rearranged $V$ genes of the Ig heavy chain. In these mice around $30 \%$ of the $B$ cells are specific for MOG and demyelinating MOG-specific antibodies can be found in the serum. Th mice do not spontaneously develop EAE, but their disease course is accelerated and exacerbated upon immunization compared to wild-type littermates (Litzenburger et al., 1998).

\subsubsection{Breeding}

All genetically modified mice were derived from in house breeding colonies at the central animal facility of the University Medical Center Goettingen. 


\subsubsection{Housing}

All mice were housed in the central animal facility of the University Medical Center Goettingen under specific pathogen free (SPF) conditions. The animals had access to food and water ad libitum and were kept on a 12/12 hour light/dark cycle. Before each experiment, mice were allowed to adapt to the new environment for seven days. Animal experiments were performed in accordance with the European Communities Council Directive of 24 November 1986 (86/EEC) and were approved by the Government of Lower Saxony, Germany (G 347.11, G 1005.12).

\subsection{Methods}

\subsubsection{Genotyping of genetically modified mice}

Genotyping of genetically modified mice was performed by Ms. Katja Schulz (Department of Neuropathology, University Medical Center Goettingen).

\section{DNA extraction}

Genomic DNA was isolated from tail biopsies of $\operatorname{Rag} 1^{--}, 2 \mathrm{D} 2$ and Th mice. For this purpose, the tissue was digested in $350 \mu$ lysis buffer with $20 \mu \mathrm{l}$ of proteinase $\mathrm{K}$ over night (350 rpm, $56^{\circ} \mathrm{C}$ ). Remaining tissue was removed by centrifugation ( $5 \mathrm{~min}, 13,200 \mathrm{rpm}, \mathrm{RT}$ ). DNA in the supernatant was precipitated by adding $350 \mu \mathrm{l}$ isopropyl alcohol. After a second centrifugation, the pellet was washed in $350 \mu \mathrm{l} 70 \%$ ethanol. After a third centrifugation, the supernatant was discarded, the pellet was dried for $10 \mathrm{~min}$ in a speed vacuum concentrator and resuspended in $100 \mu$ l bidistilled water.

\section{Genotyping of Rag1 $\underline{-t}$ mice}

$P C R$ reaction

Each reaction contained $2 \mu \mathrm{l}$ genomic DNA, $1 \mu \mathrm{l} 10 \mathrm{mM}$ dNTP Mix, $1 \mu \mathrm{l}$ of each oligonucleotide primer (oIMR1746, oIMR3104, oIMR8162), $0.2 \mu \mathrm{l}$ Go-Taq ${ }^{\circledR}$ DNA polymerase, $5 \mu \mathrm{l}$ Go-Taq $^{\circledR}$ DNA polymerase buffer $(5 x)$ and $13.8 \mu$ l bidistilled water. 
PCR program

PCR reactions were run in a T3 thermocycler at the following cycling conditions:

\begin{tabular}{lll}
\hline Temperature & Time & Step \\
\hline $94^{\circ} \mathrm{C}$ & $120 \mathrm{~s}$ \\
$94^{\circ} \mathrm{C}$ & $30 \mathrm{~s}$ \\
$58^{\circ} \mathrm{C}$ & $45 \mathrm{~s}$ \\
$72^{\circ} \mathrm{C}$ & $45 \mathrm{~s}$ \\
$72^{\circ} \mathrm{C}$ & $120 \mathrm{~s}$ \\
$4^{\circ} \mathrm{C}$ & $\infty$ & $\begin{array}{l}\text { Pre-denaturation } \\
\mathrm{w}\end{array}$ \\
\hline $\begin{array}{l}\frac{1}{D} \\
心\end{array}$ & $\begin{array}{l}\text { Denaturation } \\
\text { Ennealing } \\
\end{array}$ \\
\hline
\end{tabular}

Agarose gel electrophoresis

To analyze the PCR products, $10 \mu \mathrm{l}$ of the sample were loaded on an agarose gel ( $2 \%$ in TBE buffer) containing $3 \mu$ ethidium bromide. Electrophoresis was performed at $100 \mathrm{~V}$ for $60 \mathrm{~min}$. PCR product length was evaluated in relation to a $100 \mathrm{bp}$ DNA ladder. A band at $474 \mathrm{bp}$ was characteristic for the wild-type allele, whereas a product with a length of $530 \mathrm{bp}$ was detected in Rag1-deficient samples. Heterozygous animals displayed both bands.

\section{Genotyping of 2D2 mice}

\section{$P C R$ reaction}

Each reaction contained $5 \mu$ l genomic DNA, $0.4 \mu \mathrm{l} 10 \mathrm{mM}$ dNTP Mix, $0.5 \mu \mathrm{l}$ of each oligonucleotide primer (oIMR6711, olMR6712), $0.1 \mu$ l Go-Taq ${ }^{\circledR}$ DNA polymerase, $4 \mu$ Go-Taq $^{\circledR}$ DNA polymerase buffer $(5 x)$ and $9.5 \mu$ bidistilled water.

PCR program

$\mathrm{PCR}$ reactions were run in a T3 thermocycler at the following cycling conditions:

\begin{tabular}{|c|c|c|}
\hline Temperature & Time & Step \\
\hline $94^{\circ} \mathrm{C}$ & $120 \mathrm{~s}$ & Pre-denaturation \\
\hline $94^{\circ} \mathrm{C}$ & $60 \mathrm{~s}$ & Denaturation \\
\hline $58^{\circ} \mathrm{C}$ & $60 \mathrm{~s}$ & Annealing \\
\hline $72^{\circ} \mathrm{C}$ & $60 \mathrm{~s}$ & Elongation \\
\hline $72^{\circ} \mathrm{C}$ & $10 \min$ & Final elongation \\
\hline $4^{\circ} \mathrm{C}$ & $\infty$ & Storage \\
\hline
\end{tabular}




\section{Agarose gel electrophoresis}

To analyze the PCR products, $20 \mu \mathrm{l}$ of the sample were loaded on an agarose gel ( $2 \%$ in TBE buffer) containing $3 \mu$ lethidium bromide. Electrophoresis was performed at $120 \mathrm{~V}$ for $45 \mathrm{~min}$. PCR product length was evaluated in relation to a $100 \mathrm{bp}$ DNA ladder. Animals carrying the transgene displayed a band at $675 \mathrm{bp}$, whereas no band was detected in wild type animals.

\section{Genotyping of Th mice}

\section{$P C R$ reaction}

Each reaction contained $5 \mu$ l genomic DNA, $0.4 \mu \mathrm{l} 10 \mathrm{mM}$ dNTP Mix, $0.5 \mu \mathrm{l}$ of each oligonucleotide primer (8.18C5-sense, 8.18C5-antisense, mlgH-sense, mlgH-antisense), $0.1 \mu \mathrm{l} \mathrm{Go-}$ Taq $^{\circledR}$ DNA polymerase, $4 \mu$ l Go-Taq ${ }^{\circledR}$ DNA polymerase buffer $(5 x)$ and $8.5 \mu$ l bidistilled water.

\section{PCR program}

PCR reactions were run in a T3 thermocycler at the following cycling conditions:

\begin{tabular}{|c|c|c|}
\hline Temperature & Time & Step \\
\hline $94^{\circ} \mathrm{C}$ & $120 \mathrm{~s}$ & Pre-denaturation \\
\hline $94^{\circ} \mathrm{C}$ & $60 \mathrm{~s}$ & Denaturation \\
\hline $61^{\circ} \mathrm{C}$ & $60 \mathrm{~s}$ & Annealing \\
\hline $72^{\circ} \mathrm{C}$ & $60 s$ & Elongation \\
\hline $72^{\circ} \mathrm{C}$ & $10 \mathrm{~min}$ & Final elongation \\
\hline $4^{\circ} \mathrm{C}$ & $\infty$ & Storage \\
\hline
\end{tabular}

Agarose gel electrophoresis

To analyze the PCR products, $20 \mu \mathrm{l}$ of the sample were loaded on an agarose gel ( $2 \%$ in TBE buffer) containing $3 \mu$ l ethidium bromide. Electrophoresis was performed at $120 \mathrm{~V}$ for $45 \mathrm{~min}$. PCR product length was evaluated in relation to a $100 \mathrm{bp}$ DNA ladder. The wild type allele was characterized by a band at $150 \mathrm{bp}$, the transgenic allele by a band at $100 \mathrm{bp}$. Both bands were detected in heterozygous animals.

\subsubsection{Experimental autoimmune encephalomyelitis}

Experimental autoimmune encephalomyelitis (EAE) is the most commonly used experimental animal model to study different autoimmune aspects of MS. 


\subsubsection{EAE induction}

In this thesis, EAE was induced in $\mathrm{C} 57 \mathrm{BI} / 6 \mathrm{~J}$ and heterozygous $\mathrm{Th}(\mathrm{Th} /+)$ mice by active immunization with $\mathrm{MOG}_{35-55}$ emulgated in complete Freund's adjuvant (CFA). Mice were anesthetized with $100 \mu \mathrm{l}$ ketamine/xylazine anesthesia per $10 \mathrm{~g}$ body weight and subcutaneously immunized with $50 \mu \mathrm{MOG}_{35-55} / \mathrm{CFA}$ at the dorsal side of each limb. The amount of peptide per animal was $200 \mu \mathrm{g} \mathrm{MOG}_{35-55}$ for $\mathrm{C} 57 \mathrm{Bl} / 6 \mathrm{~J}$ mice and $50 \mu \mathrm{g}$ for Th/+ mice. On the day of immunization and at day 2 post immunization (p.i.) 400 ng pertussis toxin (PTX) in PBS were administered intraperitoneally to each mouse.

\subsubsection{Clinical EAE score}

To assess EAE severity, body weight and disease score were evaluated daily in each animal, starting at day seven after immunization until the end of the experiment. The criteria defining the clinical EAE score used in this study are summarized in TABLE 15. Animals reaching a disease score of 4 had to be sacrificed, due to ethical reasons. The final score of all sacrificed animals was carried forward until the end of the experiment, while animals which died of EAE were scored 5.

TABLE 15: Clinical EAE score

\begin{tabular}{ll}
\hline Score & Clinical correlate \\
\hline 0 & Healthy; no clinical signs \\
0.5 & Partial paralysis of the tail \\
1 & Complete paralysis of the tail \\
2 & Beginning paralysis of the hind limbs; mouse can be turned on the back \\
2.5 & Strong paralysis of the hind limbs; no self-dependent turning \\
3 & Complete paralysis of hind limbs; beginning paralysis of abdominal muscles \\
3.5 & Complete paralysis of abdominal muscles, beginning weakness of fore limbs \\
4 & Pronounced weakness or complete paralysis of fore limbs \\
5 & Dead \\
\hline
\end{tabular}

\subsubsection{LAQ treatment}

LAQ was kindly provided by Teva Pharmaceutical Industries, Ltd., Netanya, Israel. Animals received daily treatment with $25 \mathrm{mg} / \mathrm{kg}$ LAQ by oral gavage. Body weight was determined daily and $100 \mu \mathrm{LAQ}(2.5 \mathrm{mg} / \mathrm{ml}$ in water) were administered per $10 \mathrm{~g}$ body weight. Control animals received the same volume of vehicle (water). 
To analyze the therapeutic efficiency of LAQ in EAE, C57BI/6J mice and Th/+ mice were treated with LAQ or vehicle from the day of immunization until day 28 after immunization. To investigate early LAQ-induced changes within different immune cell populations in immunized animals, C57BI/6J mice received LAQ or vehicle for eleven days, starting at the day of immunization. To study the effects of $L A Q$ in naïve animals, naïve $\mathrm{C} 57 \mathrm{BI} / 6 \mathrm{~J}$ mice and Rag ${ }^{-1}$ mice were treated with $L A Q$ or vehicle for 20 days.

\subsubsection{NK cell depletion in EAE}

NK cell depletion was performed in $\mathrm{C} 57 \mathrm{BI} / 6 \mathrm{~J}$ and Th/+ mice. In immunized C57BI/6J animals the influence of NK cells on LAQ-mediated changes in dendritic cells was investigated.

Experiments in Th/+ mice assessed the relevance of NK cells for the treatment effect of LAQ in EAE. This experimental setup compared four different groups of EAE animals:

1. NK cell-competent $\mathrm{Th} /+$ mice treated with vehicle.

2. NK cell-depleted Th/+ mice treated with vehicle.

3. NK cell-competent $\mathrm{Th} /+$ mice treated with $25 \mathrm{mg} / \mathrm{kg} \mathrm{LAQ}$.

4. NK cell-depleted Th/+ mice treated with $25 \mathrm{mg} / \mathrm{kg} \mathrm{LAQ}$.

NK cells were depleted by the intraperitoneal administration of $300 \mu \mathrm{g}$ anti-NK1.1 antibody (clone PK-136) in PBS at day 2 and 1 before immunization and thereafter every other day until the end of the experiment. NK cell-competent animals received identical treatment with an isotype control antibody (clone C1.18.4). Successful NK cell depletion was controlled in the peripheral blood of all animals at the day of immunization and at the end of the experiment by flow cytometry. LAQ and vehicle were administered daily by oral gavage, starting at the day of immunization until the end of the experiment. The experimental setup is presented in FIGURE 31 (see section 3.6).

\subsubsection{Analysis of immune cells ex vivo}

To investigate LAQ-induced changes in different cell populations of the innate and adaptive immune system, different organs and tissues were isolated from LAQ- and vehicletreated animals. Single cell suspensions were prepared and immune cells were analyzed ex vivo using flow cytometry. 


\subsubsection{Preparation of single cell suspensions}

Single cell suspensions were prepared from spleen, lymph node and blood for further analysis. All centrifugation steps were performed for $10 \mathrm{~min}$ at $300 \mathrm{xg}$ and $4^{\circ} \mathrm{C}$ and cells were

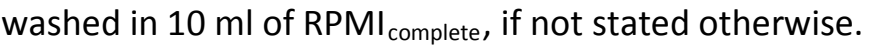

\subsection{Preparation of spleen leukocytes}

To investigate effects of $L A Q$ on splenocytes, spleens were isolated from naïve and immunized animals, treated with LAQ or vehicle in vivo.

To study effects of LAQ on T and NK cells in the spleen, single cell suspensions were obtained by mechanical dissociation. For this purpose, spleens were disrupted with a syringe plunger on a sterile $70 \mu \mathrm{m}$ cell strainer. Cells were flushed through the cell strainer by adding $10 \mathrm{ml}$ of RPMI complete and collected in a $50 \mathrm{ml}$ tube. After one washing step, splenocytes were further processed dependent on the following experiment.

For direct flow cytometric analysis of $\mathrm{T}$ and NK cells, erythrocytes were lysed by resuspending the pellet in $1 \mathrm{ml}$ of $\mathrm{BD}$ Pharm lyse ${ }^{\mathrm{TM}}$ solution (pre-diluted 1:10 in bidistilled water). Lysis was performed for $3 \mathrm{~min}$ at $37^{\circ} \mathrm{C}$ and stopped by adding $20 \mathrm{ml}$ of RPMI complete. After one washing step, cells were resuspended in $10 \mathrm{ml}$ of $\mathrm{RPMI}_{\text {complete }}$.

For further purification of T and NK cells by magnetic-activated cell sorting (MACS) and fluorescence-activated cell sorting (FACS), no erythrocyte lysis was performed. Instead, cells were washed once and were then resuspended in $5 \mathrm{ml}$ of MACS buffer.

To evaluate effects of LAQ on dendritic cells, single cell suspensions were obtained by parallel enzymatic digestion and mechanical dissociation. Spleens, isolated from LAQ- and vehicletreated animals, were transferred into a gentleMACS ${ }^{\mathrm{TM}} \mathrm{C}$-tube with $2 \mathrm{ml}$ of digestion solution (HBSS [with $\mathrm{Ca}^{2+}, \mathrm{Mg}^{2+}$ ] containing $2.5 \mathrm{mg} / \mathrm{ml}$ Collgenase $\mathrm{D}$ and $2 \mathrm{mg} / \mathrm{ml}$ DNasel). Mechanical dissociation was performed using the gentleMACS ${ }^{\text {тM }}$ Dissociator with the preprogrammed settings for murine spleen dissociation and the tissue was digested for $15 \mathrm{~min}$ at $37^{\circ} \mathrm{C}$. The obtained cell suspension was transferred onto a sterile $70 \mu \mathrm{m}$ cell strainer and single cells were flushed through by adding $10 \mathrm{ml}$ of RPMI complete. Washing and erythrocyte lysis were performed as described above.

Cell numbers were determined prior to further analyses (see 2.3.3.1.4).

\subsection{Preparation of leukocytes from the lymph nodes}

The effects of LAQ on the ability of lymph node cells to secrete IL-17 and IFNy upon ex vivo stimulation were analyzed. To this end, axillary and inguinal lymph nodes were isolated from 
animals, immunized with $M_{35}$-55 and treated with $L A Q$ or vehicle. Lymph nodes were transferred to a sterile $70 \mu \mathrm{m}$ cell strainer and mechanically disrupted using a syringe plunger.

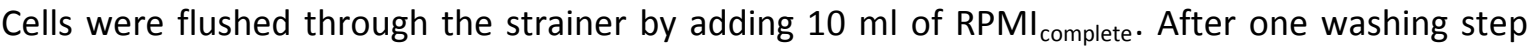
the pellet was resuspended in $3 \mathrm{ml}$ of $\mathrm{RPMI}_{\text {complete }}$ and cell numbers were determined in a Neubauer chamber (see 2.3.3.1.4).

\subsection{Preparation of peripheral blood leukocytes}

Peripheral blood leukocytes (PBMC) were isolated to confirm the efficiency of NK cell depletion in mice by flow cytometry. Blood samples were obtained by puncture of the Vena facialis. Several drops of blood were diluted in $300 \mu$ of FACS buffer containing 1 mM EDTA and were transferred to a $5 \mathrm{ml}$ FACS tube. $1 \mathrm{ml}$ of FACS buffer with $1 \mathrm{mM}$ EDTA was added and the tubes were centrifuged. Cells were resuspended in $1 \mathrm{ml}$ of FACS buffer with $1 \mathrm{mM}$ EDTA.

\subsection{Cell counting}

Cell numbers were determined in a Neubauer chamber. To exclude dead cells, the cell suspension was diluted 1:10 in trypan blue (pre-diluted 1:10 in PBS) prior to counting. Four squares with a surface of $1 \mathrm{~mm}^{2}$ and a volume $0.1 \mu \mathrm{l}$ each were counted for every sample. The concentration of cells in the original sample was calculated using FORMULA 1. Total cell numbers were extrapolated to the sample volume.

Concetration of cells $\left[\frac{\text { cells }}{\mathrm{ml}}\right]=\frac{\text { Cells }_{\text {counted }}}{4} * 10 * 10^{4}$

\section{FORMULA 1}

Calculating the concentration of cells in a given sample after cell counting in a Neubauer chamber.

\subsubsection{Flow cytometry}

The effects of LAQ on different cell populations of the innate and adaptive immune system were analyzed by flow cytometry. Single cell suspensions were stained with different combinations of fluorochrome-labeled antibodies (see TABLE 5) and cell populations were analyzed using a FACS Canto ${ }^{\mathrm{TM}}$ II and the BD FACSDiva Software 6.1.2. If not stated otherwise, the staining procedure was performed in 96 well round bottom plates, plates were centrifuged for 5 min at $300 \times g$ and $4^{\circ} \mathrm{C}$ and cells were washed with $200 \mu$ FACS buffer. 


\subsection{FACS staining procedure of splenocytes}

Splenocytes were counted using a Neubauer chamber (see 2.3.3.1.4) and up to $0.5^{*} 10^{6}$ cells were added per well. Cells were centrifuged and washed with FACS buffer. Cells were resuspended in $50 \mu$ l blocking buffer, consisting of anti-CD16/CD32 antibody diluted 1:100 in FACS buffer, and incubated for $15 \mathrm{~min}$ at $4^{\circ} \mathrm{C}$. Up to six fluorochrome-labeled antibodies per staining were diluted 1:100 in FACS buffer, $50 \mu \mathrm{l}$ of this master mix were added to each well (final dilution 1:200) and samples were incubated for $20 \mathrm{~min}$ at $4^{\circ} \mathrm{C}$. After the staining, wells were filled with 100 $\mu$ I FACS buffer and plates were centrifuged. Cells were washed and resuspended in $50 \mu$ l of the appropriate fixation buffer. Cells, receiving pure surface staining, were fixed in Cytofix ${ }^{\mathrm{TM}}$ prediluted 1:5 in FACS buffer. Fixed cells were analyzed by flow cytometry.

\subsection{FACS staining procedure of peripheral blood leukocytes}

FACS tubes with peripheral blood cells (compare 2.3.3.1.3) were centrifuged for $8 \mathrm{~min}$ at $300 \times g$ and $4^{\circ} \mathrm{C}$ and the pellets were resuspended in $100 \mu \mathrm{l}$ blocking buffer, consisting of antiCD16/CD32 antibody diluted 1:100 in FACS buffer with 1 mM EDTA. Cells were incubated for $15 \mathrm{~min}$ at $4^{\circ} \mathrm{C}$. Up to six fluorochrome-labeled antibodies per staining were diluted 1:100 in FACS buffer with $1 \mathrm{mM}$ EDTA. $100 \mu \mathrm{l}$ of this master mix were added to each tube (final dilution 1:200) and samples were incubated for $25 \mathrm{~min}$ at $4^{\circ} \mathrm{C} .1 \mathrm{ml}$ of FACS buffer with $1 \mathrm{mM}$ EDTA was added to each reaction and tubes were centrifuged for $8 \mathrm{~min}$ at $300 \times \mathrm{g}$ and $4^{\circ} \mathrm{C}$. Cells were washed in $1 \mathrm{ml}$ of FACS buffer with $1 \mathrm{mM}$ EDTA and centrifuged as mentioned before. Lysis of erythrocytes was performed by resuspending the pellet in $1 \mathrm{ml}$ of BD FACS ${ }^{\mathrm{TM}}$ lysing solution (pre-diluted 1:10 in bidistilled water) and incubating the suspension for $4 \mathrm{~min}$ at RT. Lysis was stopped by adding $4 \mathrm{ml}$ of FACS buffer. Cells were centrifuged as described before and washed in $1 \mathrm{ml}$ of FACS buffer. After a final centrifugation step, cells were resuspended in $100 \mu$ I FACS buffer and analyzed by flow cytometry.

\subsection{Intracellular detection of FoxP3}

First, surface molecules were stained on splenocytes (see 2.3.3.2.1) and cells were fixed in FoxP3 fixation concentrate diluted 1:4 in dilution buffer for FoxP3 staining for at least one hour. For the intracellular FoxP3 staining, FoxP3 permeabilization buffer (10x) was diluted 1:10 in bidistilled water. Wells were filled with $150 \mu$ of permeabilization buffer and plates were centrifuged. The anti-FoxP3 antibody was diluted 1:100 in permeabilization buffer and $100 \mu \mathrm{l}$ of the staining solution were added to each well. Cells were incubated for $35 \mathrm{~min}$ at $4^{\circ} \mathrm{C}$. Wells were filled with $150 \mu \mathrm{l}$ of Perm/Wash ${ }^{\mathrm{TM}}$ buffer and plates were centrifuged. Cells were washed once 
with $200 \mu \mathrm{l}$ of Perm/Wash ${ }^{\text {TM }}$ buffer and once with FACS buffer. After a final centrifugation step, cells were resuspended in $100-200 \mu$ of FACS buffer and analyzed by flow cytometry.

\subsection{Intracellular detection of cytokines}

To analyze the percentage of IL-17- and IFNY-producing CD4+ T cells by flow cytometry, up to $0.5^{*} 10^{6}$ splenocytes from immunized animals, treated with LAQ or vehicle in vivo, were seeded per well of a 96 well round bottom plate. Cytokine production was stimulated by adding $200 \mu$ l of

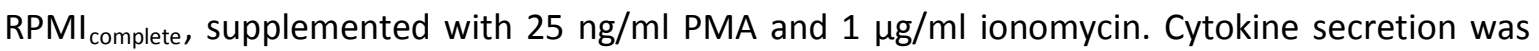
blocked by adding GolgiStop ${ }^{\mathrm{TM}}$ at a dilution of 1:1000 to the medium. Cells were incubated for $5-6 \mathrm{~h}$ at $37^{\circ} \mathrm{C}$ and $5 \% \mathrm{CO}_{2}$. Thereafter, surface staining was performed as described in 2.3.3.2.1, using FACS buffer supplemented with GolgiStop ${ }^{\text {TM }}$ (1:1000) to inhibit cytokine secretion during the staining procedure. Cells were fixed in Cytofix/Cytoperm ${ }^{\mathrm{TM}}$ for at least one hour. For the intracellular cytokine staining, Perm/Wash ${ }^{\mathrm{TM}}$ buffer (10x) was diluted 1:10 in bidistilled water. Wells were filled with $150 \mu \mathrm{l}$ of Perm/Wash ${ }^{\text {TM }}$ buffer and plates were centrifuged. Anti-IL-17 and anti-IFN $\gamma$ antibodies were diluted 1:25 in Perm/Wash ${ }^{\mathrm{TM}}$ buffer and $50 \mu \mathrm{l}$ of the staining solution were added to each well. Cells were incubated with the staining antibodies for $35 \mathrm{~min}$ at $4^{\circ} \mathrm{C}$. Wells were filled with $150 \mu \mathrm{l}$ of Perm/Wash ${ }^{\mathrm{TM}}$ buffer and plates were centrifuged. Cells were washed with FACS buffer, resuspended in $100-200 \mu \mathrm{l}$ of FACS buffer and analyzed by flow cytometry.

\subsection{Classification $T$ cells, dendritic cells and $N K$ cells according to surface and intracellular markers}

The effects of LAQ on different subpopulations of T cells, dendritic cells and NK cells were analyzed using flow cytometry. The investigated cell populations were classified according to the expression of specific surface and intracellular markers. 
T cells

TABLE 16: Classification of T cell subpopulations

\begin{tabular}{|c|c|c|c|c|c|}
\hline \multirow{2}{*}{$\begin{array}{l}\text { T cell population } \\
\text { Naïve T cells }\end{array}$} & \multicolumn{5}{|c|}{ Specific surface and intracellular markers } \\
\hline & CD19- & CD3+ & CD4+ & CD62L+ & CD44 ${ }^{\text {low }}$ \\
\hline Effector memory T cells & CD19- & CD3+ & $\mathrm{CD} 4+$ & CD62L- & CD44 $4^{\text {high }}$ \\
\hline Regulatory T cells & CD19- & CD3+ & $\mathrm{CD} 4+$ & FoxP3+ & \\
\hline Th1 cells & CD19- & $\mathrm{CD} 3+$ & $\mathrm{CD} 4+$ & $\mathrm{IFN} \gamma^{+}$ & \\
\hline Th17 cells & CD19- & $\mathrm{CD} 3+$ & $\mathrm{CD} 4+$ & IL-17+ & \\
\hline IL-17 producing $\gamma \delta$ T cells & CD19- & $\mathrm{CD} 3+$ & ү $\delta$ TCR+ & IL-17+ & \\
\hline NKT cells & CD19- & $\mathrm{CD} 3^{\mathrm{int}}$ & NK1.1 $1^{\text {int }}$ & & \\
\hline Activating receptors & DNAM-1 & TACTILE & & & \\
\hline Inhibitory receptor & TIGIT & & & & \\
\hline
\end{tabular}

\section{Abbreviations}

CD: cluster of differentiation; DNAM-1: DNAX accessory molecule-1; FoxP3: forkhead box P3; IFNY: interferon gamma; IL-17: interleukin 17; NK: natural killer; TACTILE: T cell-activated increased late expression; TCR: T cell receptor; Th1: T helper 1; Th17: T helper 17; TIGIT: T cell immunoreceptor with Ig and ITIM domains

Dendritic cells

TABLE 17: Classification of dendritic cell subpopulations

\begin{tabular}{|c|c|c|c|}
\hline \multirow{2}{*}{$\begin{array}{l}\text { Dendritic cell population } \\
\text { Dendritic cells }\end{array}$} & \multicolumn{3}{|c|}{ Specific surface markers } \\
\hline & $\mathrm{MHCll}+$ & CD11c ${ }^{\text {high }}$ & \\
\hline Myeloid dendritic cells & $\mathrm{MHCll}+$ & CD11c high & $\mathrm{CD} 11 \mathrm{~b}+$ \\
\hline Plasmacytoid dendritic cells & $\mathrm{MHCll}+$ & CD11c $c^{\text {high }}$ & B220+ \\
\hline Co-stimulatory molecules on dendritic cells & CD80 & CD86 & \\
\hline
\end{tabular}

\section{Abbreviations}

CD: cluster of differentiation; MHCII: major histocompatibility complex class II molecule 
NK cells

TABLE 18: Classification of NK cell subpopulations

\begin{tabular}{llllllll}
\hline NK cell population & \multicolumn{7}{l}{ Specific surface and intracellular markers } \\
\hline NK cells & CD19- & CD3- & NK1.1+ & NKp46+ & & \\
CD27+ NK cells & CD19- & CD3- & NK1.1+ & CD27+ & CD11b- & \\
CD27+ CD11b+ NK cells & CD19- & CD3- & NK1.1+ & CD27+ & CD11b+ & \\
CD11b+ NK cells & CD19- & CD3- & NK1.1+ & CD27- & CD11b+ & & \\
IFNץ producing NK cells & CD19- & CD3- & NK1.1+ & IFNy+ & & & \\
Activation marker & CD69 & & & & & & \\
Activating receptors & CD2 & CD244.2 & DNAM-1 & LFA-1+ & Ly49D & NKG2D & TACTILE \\
Inhibitory receptors & Ly49A & Ly49C & Ly49F & Ly49G2 & Ly49l & TIGIT & \\
\hline
\end{tabular}

\section{Abbreviations}

CD: cluster of differentiation; DNAM-1: DNAX accessory molecule-1; IFNY: interferon gamma; LFA-1: leukocyte function-associated antigen-1; NK: natural killer; NKG2D: natural killer group 2, member D; NKp46: natural killer cell p46-related protein; TACTILE: T cell-activated increased late expression

\subsubsection{Co-culture experiments}

To analyze effects of LAQ on NK cells in detail, experiments were performed to investigate NK cell effector functions in co-culture with tumor cells, T cells and dendritic cells. For this purpose, NK cells as well as CD4+ T cells and bone marrow-derived dendritic cells were purified and the B16F10 melanoma cell line was cultured. If not stated otherwise, cells were centrifuged for $10 \mathrm{~min}$ at $300 \times \mathrm{g}$ and $4^{\circ} \mathrm{C}$ and washed in a volume of $10 \mathrm{ml}$.

\subsubsection{Purification of NK cells}

NK cells were isolated from the spleens of LAQ- and vehicle-treated animals and sequentially purified by MACS and FACS sorting. In a first step, untouched NK cells were enriched by MACS and then sorted to a purity of above $99 \%$ with a cell sorter. The latter method allowed also to purify NK cell subpopulations.

Splenocytes were isolated as described in 2.3.3.1.1 and resuspended in $5 \mathrm{ml}$ of MACS buffer. The cell suspension was applied on a $40 \mu \mathrm{m}$ cell strainer and cells were flushed through the cell strainer by adding $5 \mathrm{ml}$ of MACS buffer. Cells were counted in a Neubauer chamber (see 2.3.3.1.4). NK cells were then purified using the NK cell isolation kit II (mouse), LS columns and the QuadroMACS ${ }^{\mathrm{TM}}$ separator. MACS was performed following the manufacturer's instructions. After 
MACS, cells were resuspended in $200 \mu \mathrm{l}$ of FACS buffer and transferred to a 96 well round bottom plate for the surface staining. The plate was centrifuged for $5 \mathrm{~min}$ at $300 \times \mathrm{g}$ and $4^{\circ} \mathrm{C}$ and washed once with $200 \mu \mathrm{l}$ of FACS buffer. Per well, $200 \mu \mathrm{l}$ of the staining solution were prepared, containing FACS buffer, Fc-blocking anti-CD16/CD32 antibody (1:100) and fluorochrome-labeled antibodies (1:200). After centrifugation, cells were resuspended in $200 \mu \mathrm{l}$ of the staining solution and incubated for $15 \mathrm{~min}$ at $4^{\circ} \mathrm{C}$. Cells were washed once with $200 \mu \mathrm{l}$ FACS buffer and resuspended in $300-400 \mu \mathrm{F}$ FACS buffer for cell sorting.

Total NK1.1+ CD3- NK cells or CD27+ CD11b- NK cells and CD27- CD11b+ NK cells were purified using a BD FACSAria ${ }^{\mathrm{TM}}$ II and the BD FACSDiva Software 6.1.2. After FACS sorting, NK cells were washed in $\mathrm{RPMI}_{\text {complete, }}$ counted in a Neubauer chamber (see 2.3.3.1.4) and the appropriate number of cells was put into culture (see 2.3.4.5, 2.3.4.6, 2.3.4.8, 2.3.4.9, 2.3.4.10, 2.3.5.2, 2.3.6.4 and 2.3.6.5).

\subsubsection{Purification of CD4+ T cells}

CD4+ T cells were purified from the spleens of naïve C57BI/6J and 2D2 mice using MACS. Splenocytes were isolated as described in 2.3.3.1.1 and resuspended in $5 \mathrm{ml}$ of MACS buffer. The cell suspension was applied on a $40 \mu \mathrm{m}$ cell strainer and cells were flushed through the cell strainer by adding $5 \mathrm{ml}$ of MACS buffer. Cells were counted in a Neubauer chamber (see 2.3.3.1.4). CD4+ T cells were then purified using the CD4+ T cell isolation kit II (mouse), LS columns and the QuadroMACS ${ }^{\mathrm{TM}}$ separator. MACS was performed following the manufacturer's instructions. After purification, cells were washed in $\mathrm{RPMI}_{\text {complete }}$ and counted in a Neubauer chamber (see 2.3.3.1.4). The appropriate cell number was either directly put into culture (see 2.3.4.9) or T cells were stained with CFSE (see 2.3.4.11) for assessment of in vitro proliferation (see 2.3.4.8, 2.3.4.9 and 2.3.4.10). The purity of CD4+ $\mathrm{T}$ cells was controlled by flow cytometry.

\subsubsection{Generation of bone marrow-derived dendritic cells}

Bone marrow was isolated from naïve $\mathrm{C} 57 \mathrm{Bl} / 6 \mathrm{~J}$ mice for the subsequent in vitro differentiation of bone marrow-derived dendritic cells. Therefore, hind limbs were isolated and muscle, cartilage and sinews were completely removed from bones. Femur and tibia were washed in a petri dish with RPMI complete. Bones were opened at both ends and bone marrow was flushed

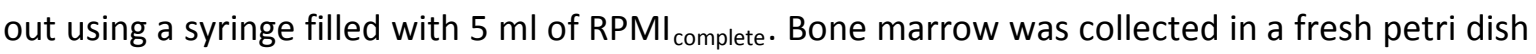
and a single cell suspension was obtained by pipetting the cell suspension up and down with a 1 $\mathrm{ml}$ pipette. The single cell suspension was then transferred onto a sterile $70 \mu \mathrm{m}$ cell strainer and cells were flushed through by adding $10 \mathrm{ml}$ of RPMI complete. Thereafter, cells were centrifuged and 
washed twice. Finally, the pellet was resuspended in $10 \mathrm{ml}$ of RPMI complete per donor mouse and the cell number was determined in a Neubauer chamber (see 2.3.3.1.4).

For the in vitro differentiation of dendritic cells, bone marrow cells were diluted to a concentration of $10^{6}$ cells per $\mathrm{ml}$ in RPMI complete containing $25 \mathrm{ng} / \mathrm{ml} \mathrm{GM}-\mathrm{CSF} .4 .5 \mathrm{ml}$ of the cell suspension were added per well of a 6 well plate. Cells were cultured in this differentiation medium for 7 days $\left(37^{\circ} \mathrm{C}, 5 \% \mathrm{CO}_{2}\right)$. At day 3 and day 5 of the culture, $2.5 \mathrm{ml}$ medium per well were replaced by fresh $\mathrm{RPMI}_{\text {complete }}$ containing $25 \mathrm{ng} / \mathrm{ml} \mathrm{GM-CSF}$. After 7 days of differentiation, the cells were harvested, washed in MACS buffer and counted in a Neubauer chamber (see 2.3.3.1.4). Bone marrow-derived dendritic cells were then purified using CD11c MicroBeads (mouse), LS columns and the QuadroMACS ${ }^{\mathrm{TM}}$ separator. MACS was performed following the manufacturer's instructions. After purification, dendritic cells were washed in $\mathrm{RPMI}_{\text {complete }}$ and counted in a Neubauer chamber (see 2.3.3.1.4). The appropriate number of cells was seeded in a 96 well flat bottom plate and dendritic cells were allowed to adhere over night. For experiments with LPS exposure, dendritic cell maturation was induced by supplementing the medium with 50 $\mathrm{ng} / \mathrm{ml}$ LPS during this overnight culture.

The purity of bone marrow-derived dendritic cells was controlled by flow cytometry.

\subsubsection{Culture of B16F10 cells}

Cryo-stocks of the melanoma cell line B16F10 (Fidler, 1973, Fidler, 1975) were kindly provided by Prof. Evelyn Ullrich (Children's Hospital, Department of Pediatric Hematology and Oncology, Goethe-University, Frankfurt, Germany). Cells were thawed at $37^{\circ} \mathrm{C}$ and transferred into $10 \mathrm{ml}$ of $\mathrm{RPMI}_{\text {complete. }}$. After one washing step in $\mathrm{RPMI}_{\text {complete, }}$ cells were resuspended in $15 \mathrm{ml}$ of $\mathrm{RPMI}_{\text {complete, }}$ seeded in a $75 \mathrm{~cm}^{2}$ cell culture flask and incubated at $37^{\circ} \mathrm{C}$ and $5 \% \mathrm{CO}_{2}$.

Passaging of the melanoma cells was first performed when cells reached confluency and then every second or third day. Adherent cells were washed once with $10 \mathrm{ml}$ of PBS. Cell adhesion was disrupted by adding $1.5 \mathrm{ml}$ of $0.01 \%$ trypsin in PBS for $2-5 \mathrm{~min}$ at room temperature and cells were loosened by gently tapping on the flask. Trypsinization was stopped by adding $8.5 \mathrm{ml}$ of $\mathrm{RPMI}_{\text {complete }}$ to the cell culture flask. $1 \mathrm{ml}$ of the cell suspension was transferred to a new flask and

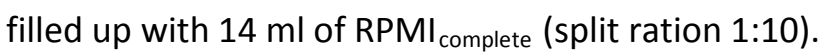

Cryo-stocks of B16F10 melanoma cells were prepared as follows. Cells were harvested as described above, washed in $\mathrm{RPMI}_{\text {complete }}$ and counted in a Neubauer chamber (see 2.3.3.1.4). $5 * 10^{6}$ cells were dissolved in $1 \mathrm{ml}$ of $\mathrm{RPMI}_{\text {complete }}$ with $10 \%$ DMSO and transferred to a cryogenic tube. Cells were frozen in a freezing container at $-80^{\circ} \mathrm{C}$ over night. For optimal cryopreservation, cryogenic tubes were stored in liquid nitrogen. 


\subsubsection{Co-culture of NK cells with B16F10 cells}

The effects of LAQ were investigated on the killing efficiency of NK cells towards NK cellsensitive tumor cells in vitro. B16F10 melanoma cells (target) were harvested (see 2.3.4.4), washed in $\mathrm{RPMI}_{\text {complete, }}$ counted in a Neubauer chamber (see 2.3.3.1.4) and 5,000 cells were seeded per well of a 96 well flat bottom plate. Splenocytes were isolated (see 2.3.3.1.1) from mice, immunized with $\mathrm{MOG}_{35-55}$ and treated with $\mathrm{LAQ}$ or vehicle in vivo. Total NK cells, CD27+ NK cells and CD11b+ NK cells (effector) were purified (see 2.3.4.1) and added to the tumor cells at different effector/target ratios, ranging from 5:1 to 0.5:1 as described in the literature (Chan et al., 2010, Mizutani et al., 2012, Cui et al., 2013). NK and tumor cells were co-cultured in

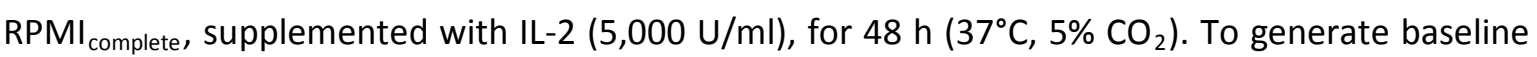
values for tumor cell survival, 5,000 tumor cells were cultured without NK cells. Samples were run in triplicates. The survival of tumor cells was assessed by crystal violet staining (see 2.3.4.7).

\subsubsection{Co-culture of NK cells with bone marrow-derived dendritic cells}

To evaluate whether NK cells directly killed bone marrow-derived dendritic cells in vitro, splenic NK cells were purified (see 2.3.3.1.1 and 2.3.4.1) from mice, immunized with $\mathrm{MOG}_{35-55}$ and treated with LAQ or vehicle for eleven days in vivo. Bone marrow-derived dendritic cells were generated as described above (see 2.3.4.3), 50,000 dendritic cells were seeded per well of a 96 well flat bottom plate and $50 \%$ of the wells were treated with $50 \mathrm{ng} / \mathrm{ml}$ LPS over night. Dendritic cells were washed with $\mathrm{RPMI}_{\text {complete }}$ and 50,000 NK cells in RPMI $\mathrm{Pomplete}_{\text {, supplemented }}$ with IL-2 $(5,000 \mathrm{U} / \mathrm{ml})$ were added per well. To generate baseline values for dendritic cell survival, 50,000 dendritic cells were cultured without NK cells. Samples were run in triplicates. NK cells and bone marrow-derived dendritic cells were co-cultured for $48 \mathrm{~h}\left(37^{\circ} \mathrm{C}, 5 \% \mathrm{CO}_{2}\right)$ and dendritic cell survival was assessed by crystal violet staining (see 2.3.4.7).

\subsubsection{Assessment of NK cell mediated lysis of B16F10 cells and dendritic cells by crystal violet staining}

The cytotoxicity of NK cells towards tumor cells and bone marrow-derived dendritic cells in vitro was assessed using the crystal violet assay (Bonnotte et al., 2001).

For this purpose, NK cells were co-cultured with the respective target cells as described above (see 2.3.4.5 and 2.3.4.6). After $48 \mathrm{~h}$ of co-culture, tumor cells were washed with $200 \mu \mathrm{l} 0.9 \% \mathrm{NaCl}$ solution, bone marrow-derived dendritic cells with $200 \mu \mathrm{RPMI}_{\text {complete, }}$ to remove dead and nonadherent cells. Wells were emptied by tapping the plate firmly against paper towels. Cells were fixed in $200 \mu$ l of $100 \%$ ethanol for 10 min at RT. Wells were emptied by tapping the plate firmly 
against paper towels. Cells were stained with $50 \mu$ lof crystal violet solution for 5 min at RT. Wells were gently washed with water to remove excess crystal violet solution and were emptied by tapping the plate firmly against paper towels. Cells were lysed in $100 \mu \mathrm{l}$ of $33 \%$ acetic acid and the optical density (OD) was determined at $540 \mathrm{~nm}$ using the iMark $^{\mathrm{TM}}$ microplate reader. The proportion of viable cells was calculated using FORMULA 2, the proportion of specific lysis was calculated using FORMULA 3.

Viable cells $[\%]=\frac{O D_{\text {experiment }}}{O D_{\text {baseline }}} * 100$

\section{FORMULA 2}

Calculating the percentage of viable cells remaining after the incubation with NK cells.
Specific lysis $[\%]=\left(1-\frac{O D_{\text {experiment }}}{O D_{\text {baseline }}}\right) * 100$

\section{FORMULA 3}

Calculating the percentage of specific lysis induced by NK cells upon co-culture with target cells.

\subsubsection{Co-culture of NK cells with CD4+ T cells}

The effect of NK cells (treated with LAQ or vehicle in vivo) on antigen-independent T cell proliferation was analyzed in vitro. Splenic NK cells were purified (see 2.3.3.1.1 and 2.3.4.1) from mice, immunized with $\mathrm{MOG}_{35-55}$ and treated with $L A Q$ or vehicle for eleven days in vivo. Splenic T cells were purified (see 2.3.3.1.1 and 2.3.4.2) from naïve C57BI/6J mice and were labeled with CFSE (see 2.3.4.11). 50,000 NK cells and 100,000 CFSE-labeled T cells were seeded in $200 \mu \mathrm{l}$ $\mathrm{RPMI}_{\text {complete }}$ per well of a 96 well round bottom plate. T cell proliferation was induced by agonistic anti-CD3 antibody (4 $\mathrm{gg} / \mathrm{ml}$, pre-coated) and anti-CD28 antibody $(1 \mu \mathrm{g} / \mathrm{ml}$, soluble) and cells were cultured for $72 \mathrm{~h}\left(37^{\circ} \mathrm{C}, 5 \% \mathrm{CO}_{2}\right)$. Finally, cells were stained for T cell and NK cell markers (see 2.3.3.2.1) and the CFSE profile was measured by flow cytometry.

\subsubsection{Co-culture of NK cells with bone marrow-derived dendritic cells and CD4+ T cells}

The triple co-culture system of NK cells, T cells and bone marrow-derived dendritic cells was used to evaluate effects of NK cells, pre-treated with LAQ or vehicle in vivo, on antigendependent T cell proliferation and T and dendritic cell survival.

Bone marrow-derived dendritic cells were generated as described above (see 2.3.4.3), 50,000 cells were seeded per well of a 96 well flat bottom plate and maturation was induced by incubating the dendritic cells in $\mathrm{RPMI}_{\text {complete, }}$ supplemented with LPS $(50 \mathrm{ng} / \mathrm{ml})$, over night $\left(37^{\circ} \mathrm{C}\right.$, $5 \% \mathrm{CO}_{2}$ ). Splenic NK cells were purified (see 2.3.3.1.1 and 2.3.4.1) from mice, immunized with $M_{35-55}$ and treated with LAQ or vehicle for eleven days in vivo. 50,000 NK cells per well were 
added to the bone marrow-derived dendritic cells. Splenic CD4+ T cells were purified (see 2.3.3.1.1 and 2.3.4.2) from naïve 2D2 mice. To assess NK cell effects on T cell proliferation, 2D2 T cells were labeled with CFSE (see 2.3.4.11). To analyze NK cell-mediated killing of T cells and dendritic cells, 2D2 T cells remained unlabeled. 100,000 T cells were added to the wells, containing 50,000 NK cells and 50,000 bone marrow-derived dendritic cells. To induce T cell proliferation, the culture medium (RPMI complete) was supplemented with $\mathrm{MOG}_{35-55}(20 \mu \mathrm{g} / \mathrm{ml})$. Samples were run in triplicates. Baseline values for proliferation and cell death were measured in stimulated samples without NK cells. Spontaneous proliferation was controlled in samples without stimulation. NK cell dependent cell death of T cells and dendritic cells was evaluated after $18 \mathrm{~h}$ of co-culture by Annexin $\mathrm{V}$ and 7-AAD staining (see 2.3.4.12). NK cell effects were assessed on the $\mathrm{T}$ cell proliferation after $72 \mathrm{~h}$ of co-culture by analyzing the CFSE profile (see 2.3.4.11).

\subsubsection{Transwell experiments}

To analyze whether the inhibition of T cell proliferation by NK cells was cell contactdependent, the triple co-culture experiments were repeated in 96 well transwell plates. In these plates, each well consisted of two compartments, separated by a polyester membrane with $1 \mu \mathrm{m}$ pore size, allowing diffusion of soluble factors, but inhibiting cell contact.

Bone marrow-derived dendritic cells were generated as described above (see 2.3.4.3), 20,000 cells per well were seeded on the transwell membrane of a 96 well transwell plate and maturation was induced by incubating the dendritic cells in RPMI complete, supplemented with LPS $(50 \mathrm{ng} / \mathrm{ml})$, over night $\left(37^{\circ} \mathrm{C}, 5 \% \mathrm{CO}_{2}\right)$. Splenic T cells were purified (see 2.3.3.1.1 and 2.3.4.2) from naïve 2D2 mice and were labeled with CFSE (see 2.3.4.11). 40,000 CFSE-labeled 2D2 T cells were added to each well of the transwell insert. Splenic NK cells were purified (see 2.3.3.1.1 and 2.3.4.1) from mice, immunized with $M O_{35-55}$ and treated with $L A Q$ or vehicle for eleven days in vivo. 20,000 NK cells per well were added either to the lower compartment (inhibiting direct contact) or to the transwell insert (allowing direct contact). To induce T cell proliferation, the culture medium (RPMI complete) was supplemented with $\mathrm{MOG}_{35-55}(20 \mu \mathrm{g} / \mathrm{ml})$. Samples were run in triplicates. Baseline proliferation was measured in stimulated samples without NK cells. Spontaneous proliferation was controlled in samples without stimulation. The NK cell effects on the $\mathrm{T}$ cell proliferation were assessed after $72 \mathrm{~h}$ of co-culture by analyzing the CFSE profile using flow cytometry (see 2.3.4.11). 


\subsubsection{Analysis of T cell proliferation by CFSE dilution}

The effect of LAQ-treated NK cells on T cell proliferation in vitro was assessed by CFSEstaining of CD4+ T cells and subsequent flow cytometric analysis of CFSE dilution. CFSE penetrates the intact cell membrane and becomes irreversibly captured within the cell through a stable cross linking to intracellular proteins. Upon proliferation, the fluorescence intensity is halved at each cell division, since the cytoplasm is shared between the two daughter cells. The CFSE profile of dividing cells can be analyzed by flow cytometry (Parish, 1999). CD4+ T cells were isolated from the spleen of naïve C57BI/6J or 2D2 mice (see 2.3.3.1.1) and purified by MACS separation (see 2.3.4.2). CD4+ $T$ cells were counted in a Neubauer chamber (see 2.3.3.1.4) and cells were resuspended in CFSE buffer at a concentration of $10^{6}$ cells $/ \mathrm{ml}$. The CFSE stock solution ( $5 \mathrm{mM}$ ) was diluted $1: 1,000$ to a final concentration of $5 \mu \mathrm{M}$ in the cell suspension. Cells were then incubated with CFSE for $10 \mathrm{~min}$ at $37^{\circ} \mathrm{C}$ in the dark. The staining reaction was stopped with the 4 -fold amount of cold $\mathrm{RPMI}_{\text {complete }}$ and the cells were incubated for $5 \mathrm{~min}$ on ice. The cells were centrifuged for $10 \mathrm{~min}$ at $300 \times \mathrm{g}$ and $4^{\circ} \mathrm{C}$, the supernatant was discarded and the cells were washed in $10 \mathrm{ml}$ of RPMI complete. This washing procedure was repeated twice, for a total of three

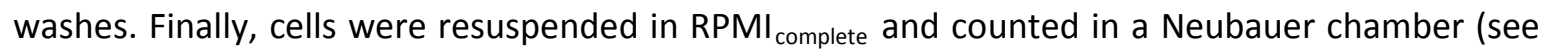
2.3.3.1.4). The appropriate number of CFSE-labeled CD4+ T cells was then used in cultures for different proliferation experiments (see 2.3.4.8, 2.3.4.9 and 2.3.4.10). T cell proliferation was analyzed by measuring the CFSE dilution using flow cytometry.

\subsubsection{Detection of apoptosis via Annexin V and 7-AAD staining}

To analyze whether NK cells isolated from LAQ-treated and control animals triggered cell death of activated $\mathrm{T}$ cells and bone marrow-derived dendritic cells in a triple co-culture system (see 2.3.4.9), Annexin $\mathrm{V}$ and 7-AAD staining was performed. Annexin $\mathrm{V}$ binds to phosphatidylserine and is a marker for early and late apoptotic/dead cells. 7-AAD is an intercalating substance that binds to GC regions of the DNA and strongly labels late apoptotic/dead cells. Both molecules do not permeate viable cells (Vermes et al., 1995, Philpott et al., 1996, Zhang et al., 1997).

To detect cell death in CD4+ T cells and bone marrow-derived dendritic cells from the triple co-culture, cells were resuspended and transferred to a 96 well round bottom plate. Wells of the original plate were flushed with ice cold $0.9 \% \mathrm{NaCl}$ solution to detach bone marrow-derived dendritic cells, which were then also transferred to the new plate. Annexin $\mathrm{V}$ binding buffer (10x) was diluted 1:10 in bidistilled water. The plate was centrifuged and cells were washed once in PBS and once in Annexin $\mathrm{V}$ binding buffer. The staining solution was prepared as follows: Annexin $\mathrm{V}$ 
binding buffer with anti-CD16/CD32 antibody (1:100), fluorochrome-labeled antibodies against CD3, NK1.1, CD11C and MHCII (1:200) and APC-conjugated Annexin V (1:20). Per well, $100 \mu \mathrm{l}$ of the staining solution were added and cells were incubated for 15 min at RT protected from light. After the staining, cells were washed once in $200 \mu$ Annexin V binding buffer and were finally resuspended in $100 \mu \mathrm{l}$ Annexin $V$ binding buffer. The plate was set on ice, $2.5 \mu \mathrm{l}$ of 7-AAD solution were added per well and after $10-15$ min Annexin $V$ and 7-AAD staining was analyzed by flow cytometry.

\subsubsection{In vitro stimulation of cytokine secretion}

To analyze the effects of LAQ on cytokines in T cells and NK cells, splenocytes and purified NK cells were stimulated ex vivo and cytokine production was measured.

\subsubsection{Stimulation of $\mathrm{T}$ cells}

To investigate effects of LAQ on the production of pro-inflammatory cytokines in T cells, lymph node cells were isolated (see 2.3.3.1.2) from animals, immunized with $\mathrm{MOG}_{35-55}$ and treated with $L A Q$ or vehicle for eleven days in vivo.

To evaluate cytokine secretion over time, lymph node cells were resuspended at a concentration of $2 * 10^{6}$ cells per $\mathrm{ml}$ in $\mathrm{HL}-1^{\mathrm{TM}}$ serum-free medium. $1 \mathrm{ml}$ of the cell suspension was transferred to each well of a 24 well plate and cytokine secretion was stimulated by adding $10 \mu \mathrm{g} / \mathrm{ml}$ of $\mathrm{MOG}_{35-55}$. Lymph node cells were incubated with $\mathrm{MOG}_{35-55}$ for $72 \mathrm{~h}$ at $37^{\circ} \mathrm{C}$ and $5 \% \mathrm{CO}_{2}$. IL-17 and IFNY concentrations in the supernatant of the culture were measured by ELISA (see 2.3.5.3).

The baseline of cytokine production was assessed in unstimulated samples.

\subsubsection{Stimulation of NK cells}

Flow cytometry and ELISA were applied to assess effects of LAQ on the capacity of NK cells to produce IFN $\gamma$. For this purpose, splenocytes (see 2.3.3.1.1) were isolated from mice, immunized with $\mathrm{MOG}_{35-55}$ and treated with LAQ or vehicle for eleven days in vivo. NK cells were purified as described in 2.3.4.1. 50,000 cells were seeded per well of a 96 well round bottom plate. To stimulate cytokine production, cells were cultured in $\mathrm{RPMI}_{\text {complete, }}$ supplemented with IL-12 $(1 \mathrm{ng} / \mathrm{ml})$ and IL-18 $(25 \mathrm{ng} / \mathrm{ml})$, over night $\left(37^{\circ} \mathrm{C}, 5 \% \mathrm{CO}_{2}\right)$. After overnight culture, plates were centrifuged for $5 \mathrm{~min}$ at $300 \times \mathrm{g}$ and $4^{\circ} \mathrm{C}$. The supernatant was removed and IFN $\gamma$ concentrations

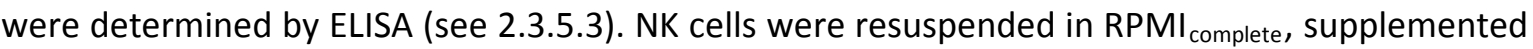


with IL-12 $(1 \mathrm{ng} / \mathrm{ml})$, IL-18 $(25 \mathrm{ng} / \mathrm{ml})$ and GolgiStop ${ }^{\mathrm{TM}}(1 / 1,000)$ and incubated for $5-6 \mathrm{~h}\left(37^{\circ} \mathrm{C}\right.$, $5 \% \mathrm{CO}_{2}$ ). NK cell surface staining (see 2.3.3.2.1) and intracellular IFN $\gamma$ staining (see 2.3.3.2.4) were performed and the frequency of IFN $\gamma+$ NK cells was analyzed by flow cytometry. The baseline of the cytokine production was assessed in unstimulated samples.

\subsubsection{Detection of cytokines using ELISA}

T cells and NK cells are potent producers of a variety of cytokines. LAQ-effects on this effector function were assessed by measuring cytokine concentrations in the supernatant of stimulated lymph node cells and purified NK cells using ELISA.

Lymph node cells were stimulated as described in 2.3.5.1 and IL-17 and IFNy secretion was analyzed. Purified NK cells were stimulated as described in 2.3.5.2 and IFNץ production was evaluated.

ELISA was performed using the suitable DuoSet $^{\circledR}$ ELISA Development System and following the manufacturer's instructions. The OD was determined at $450 \mathrm{~nm}$ with $540 \mathrm{~nm}$ wavelength correction using the iMark ${ }^{\mathrm{TM}}$ microplate reader.

\subsubsection{In vitro LAQ treatment}

T cells, bone marrow-derived dendritic cells and NK cells were treated with LAQ in vitro to differentiate between direct and indirect effects of LAQ on these cells.

\subsubsection{In vitro $\mathrm{LAQ}$ treatment of $\mathrm{T}$ cells}

To investigate whether T cells were directly modulated by LAQ, T cells were treated with $\mathrm{LAQ}$ in vitro. For this purpose, splenocytes (see 2.3.3.1.1) were isolated from C57BI/6J mice immunized with $\mathrm{MOG}_{35-55}$. Splenocytes were seeded in a 96 well round bottom plate and resuspended in $200 \mu \mathrm{l}$ of $\mathrm{RPMI}_{\text {complete. }} \mathrm{LAQ}$ was added at a concentration of 0 or $5 \mu \mathrm{M}$ to the culture and splenocytes were incubated for $72 \mathrm{~h}\left(37^{\circ} \mathrm{C}, 5 \% \mathrm{CO}_{2}\right)$. Thereafter, the frequencies of Th1 and Th17 cells were assessed upon PMA and ionomycin stimulation.

\subsubsection{Intracellular detection of pro-inflammatory cytokines in T cells after in vitro LAQ treatment}

The frequencies of Th1 and Th17 cells were used as readout for the efficiency of in vitro LAQ treatment to reduce pro-inflammatory $\mathrm{T}$ cell subsets. Therefore, after the $72 \mathrm{~h}$ incubation period with LAQ (see 2.3.6.1) splenocytes were stimulated with PMA and ionomycin and the 
frequencies of Th1 and Th17 cells were analyzed by flow cytometry after intracellular cytokine staining (see 2.3.3.2.4).

\subsubsection{In vitro LAQ treatment of bone marrow-derived dendritic cells}

To analyze whether LAQ-treated bone marrow-derived dendritic cells could induce NK cell activation upon co-culture, bone marrow-derived dendritic cells were generated in the presence of $L A Q$. For this purpose the differentiation medium was supplemented with $L A Q$ at a concentration of $10 \mu \mathrm{M}$ and fresh LAQ was added with each medium exchange. Apart from the addition of LAQ to the culture, bone marrow-derived dendritic cells were generated and purified as described above (see 2.3.4.3). 50,000 dendritic cells were seeded per well of a 96 well flat bottom plate and were allowed to adhere over night. No LPS was added to these cultures. LAQtreated bone marrow-derived dendritic cells were subsequently co-cultured with NK cells (see 2.3.6.5).

\subsubsection{In vitro LAQ treatment of NK cells}

To investigate whether NK cells were a direct target of LAQ therapy, purified NK cells were treated with LAQ in vitro. For this purpose, splenocytes (see 2.3.3.1.1) were isolated from naïve C57BI/6J mice and NK cells were purified (see 2.3.4.1). 50,000 NK cells were seeded per well of a 96 well round bottom plate and resuspended in $200 \mu \mathrm{l}$ of RPMI complete, supplemented with IL-2 $(1000 \mathrm{U} / \mathrm{ml})$. LAQ was added at a concentration of 0 or $10 \mu \mathrm{M}$ to the culture and NK cells were incubated for $48 \mathrm{~h}\left(37^{\circ} \mathrm{C}, 5 \% \mathrm{CO}_{2}\right)$. Direct effects of LAQ on NK cells were determined by analyzing the upregulation of the activation marker CD69.

\subsubsection{Co-culture of LAQ pre-treated bone marrow-derived dendritic cells with NK cells}

NK cells were co-cultured with LAQ-treated bone marrow-derived dendritic cells, to assess whether pre-treatment of bone marrow-derived dendritic cells with LAQ and subsequent coculture with NK cells could mimic direct effects of LAQ on NK cells. Splenic NK cells were purified (see 2.3.3.1.1 and 2.3.4.1) from naïve C57BI/6J animals and 50,000 NK cells in $\mathrm{RPMI}_{\text {complete, }}$ supplemented with IL-2 $(1,000 \mathrm{U} / \mathrm{ml})$, were added per well. NK cells were cultured alone or cocultured with 50,000 bone marrow-derived dendritic cells either pre-treated with $10 \mu \mathrm{M} L A Q$ (see 2.3.6.3) or not for $48 \mathrm{~h}\left(37^{\circ} \mathrm{C}, 5 \% \mathrm{CO}_{2}\right)$. 


\subsubsection{Assessment of NK cell activation by CD69 staining}

The effect of direct in vitro LAQ treatment on NK cells, as well as the effect of co-culture with pre-treated bone marrow-derived dendritic cells on NK cells was assessed by measuring NK cell activation. Therefore, the surface marker CD69 was stained on NK cells derived from the cultures described in 2.3.6.4 and 2.3.6.5 and its expression was analyzed by flow cytometry (see 2.3.3.2.1).

\subsubsection{Data analysis and statistics}

The software FlowJo 7.6.1 was used to analyze all flow cytometric data.

Statistics were calculated using the software GraphPad Prism 5.01. Data were tested for normal distribution with the help of the Kolmogorov-Smirnov test with Dallal-Wilkinson-Lilliefor $p$ value. To compare two experimental groups, unpaired t-tests were used for parametric data and Mann-Whitney $U$ tests for non-parametric data. To compare three or more groups, one way ANOVA with Bonferroni post test was performed for parametric data and the Kruskal-Wallis test with Dunn's post test was applied for non-parametric data. Survival analysis was calculated with the log-rank test.

All the statistical analyses of EAE scores after NK cell depletion in vivo (compare 3.6.2) were performed in SAS 9.3 by Mr. Markus Harden (Department for Medical Statistics, University Medical Center Goettingen, Germany) using non-parametric rank-based ANOVA-type statistics.

Statistical significance was defined as $p<0.05$. Data in text and figures are presented as mean \pm SEM. 


\section{Results}

\subsection{Preventive LAQ treatment blocked EAE induction and the development of auto-reactive T cells in vivo}

The new immunomodulatory substance $L A Q$ is under development for the treatment of multiple sclerosis. The present work aimed to analyze the effects of this new compound on innate and adaptive immunity in mice with experimental autoimmune encephalomyelitis, the most common animal model, to study different autoimmune components of multiple sclerosis.

To first approach LAQ-mediated alterations of adaptive immunity, the initial set of experiments tested the efficiency of LAQ to inhibit acute EAE in C57BI/6J mice and evaluated the effects of LAQ on pro- and anti-inflammatory T cell subpopulations. In detail, the effects of LAQ on T cells were assessed by analyzing the proportion of naïve and effector memory $T$ cells as well as the balance between anti-inflammatory regulatory T cells and pro-inflammatory Th1 and Th17 cells.

\subsubsection{Preventive LAQ treatment completely inhibited acute EAE in C57BI/6J mice}

To investigate the effect of preventive $L A Q$ treatment on $E A E, C 57 \mathrm{BI} / 6 \mathrm{~J}$ mice were immunized with $\mathrm{MOG}_{35-55}$ and received daily oral treatment with either $25 \mathrm{mg} / \mathrm{kg} \mathrm{LAQ}$ or vehicle for 28 days, starting at the day of immunization.

Control animals developed first clinical signs at day nine and clinical symptoms reached the peak of disease around days $15-20$. In contrast all LAQ-treated animals remained healthy and did not develop any EAE symptoms (FIGURE 2).

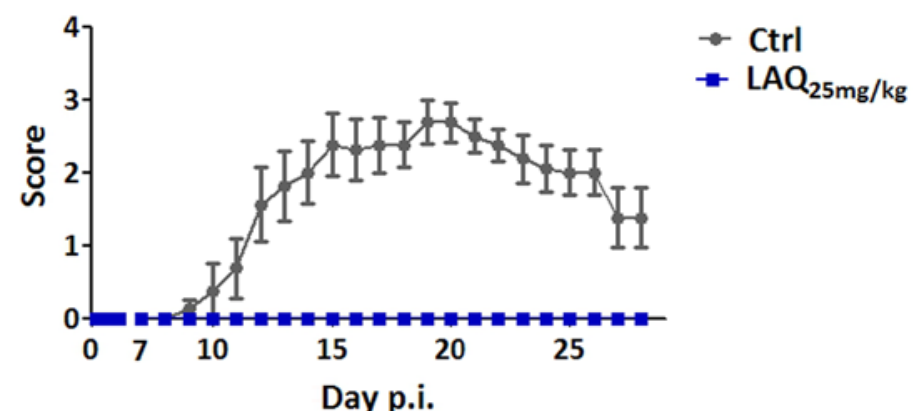

FIGURE 2: LAQ treatment protects C57BI/6J mice from $\mathrm{MOG}_{35-55}$-induced EAE

Clinical disease scores of acute EAE in C57BI/6J mice, immunized with $\mathrm{MOG}_{35-55}$ and treated with $25 \mathrm{mg} / \mathrm{kg}$ LAQ (blue) or vehicle (gray) from day 0

until day 28 after immunization. LAQ completely inhibits EAE symptoms in all treated animals $(n=8)$, whereas control animals $(n=8)$ develop first clinical signs at day nine after immunization, whereby clinical symptoms peak around day $15-20$ after immunization. Data are presented as mean \pm SEM. 


\subsubsection{LAQ treatment increased naïve and decreased effector memory $T$ cells}

LAQ-induced changes in the frequencies of naïve and effector memory $T$ cells were analyzed using splenocytes from MOG $35-55$-immunized mice treated with $25 \mathrm{mg} / \mathrm{kg} \mathrm{LAQ}$ or vehicle for eleven days. Naïve and effector memory $T$ cells were distinguished in the flow cytometric analysis by their expression levels of the surface markers CD62L and CD44. Naïve T cells were characterized as CD62L+CD44 ${ }^{\text {low }} T$ cells, whereas effector memory $T$ cells were defined as CD62LCD44 ${ }^{\text {high }}$ T cells (FIGURE 3A).

The frequency of naïve T cells was markedly increased in LAQ-treated animals (mean $66.3 \% \pm 1.2 \%$ ) compared to control animals (mean $55.7 \% \pm 3.7 \%, p<0.05$ ) (FIGURE 3B). In contrast, LAQ-treated animals (mean $20.6 \% \pm 0.8 \%$ ) displayed a significantly lower frequency of effector memory T cells than controls (mean $32.8 \% \pm 3.5 \%$, p<0.01) (FIGURE 3C).

A

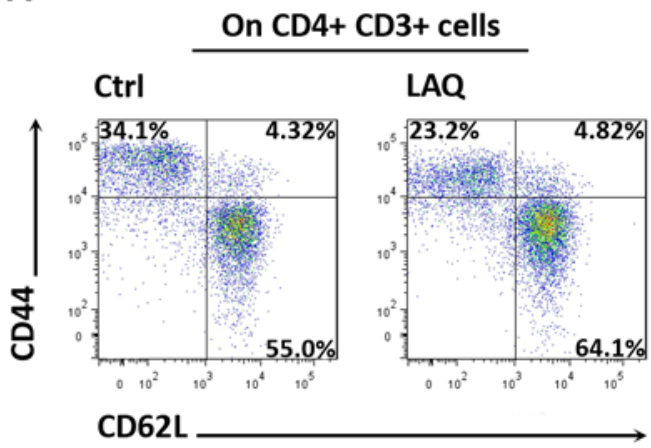

B

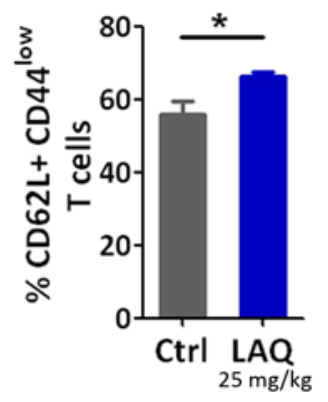

C

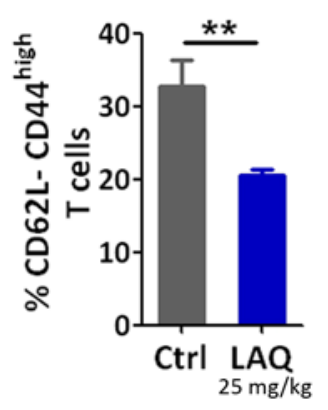

FIGURE 3: LAQ augments naïve and reduces effector memory $T$ cells

The frequencies of CD62L+ CD44 ${ }^{\text {low }}$ naïve $T$ cells and CD62L- CD44 ${ }^{\text {high }}$ effector memory $T$ cells were evaluated by flow cytometry (A). Splenocytes were isolated from animals immunized with $\mathrm{MOG}_{35-55}$ and treated with $25 \mathrm{mg} / \mathrm{kg}$ LAQ (blue) or vehicle (gray) for eleven days. In vivo LAQ treatment significantly increases the frequency of naïve T cells compared to controls ( $n=6$ per group, ${ }^{*} p<0.05$, unpaired t-test) (B). In contrast, the frequency of effector memory $T$ cells is decreased in LAQ-treated animals compared to vehicle-treated controls ( $n=6$ per group, ${ }^{* *} p<0.01$, Mann-Whitney $U$ test) (C). Data are presented as mean \pm SEM. 


\subsubsection{LAQ treatment decreased pro-inflammatory $\mathrm{T}$ cell subsets}

To evaluate the effects of LAQ treatment on pro-inflammatory T cell subsets, the production of the pro-inflammatory cytokines IL-17 and IFNY was measured upon ex vivo stimulation of cells isolated from $\mathrm{MOG}_{35-55}$-immunized mice pre-treated with $25 \mathrm{mg} / \mathrm{kg} \mathrm{LAQ}$ or vehicle for eleven days in vivo.

\subsubsection{LAQ reduced IL-17-producing CD4+ and $\gamma \delta \mathrm{T}$ cells}

To assess effects of LAQ on the frequency of IL-17-producing CD4+ T cells (Th17 cells) and IL-17-producing $\gamma \delta T$ cells, splenocytes from LAQ-treated or control animals were stimulated with PMA and ionomycin ex vivo. Cytokine secretion was blocked and intracellular IL-17 staining was analyzed by flow cytometry (FIGURE 4A\&C).

Flow cytometry revealed a significant decrease in the frequency of Th17 cells in LAQtreated animals (mean $0.3 \% \pm 0.05 \%$ ) compared to control animals (mean $3.7 \% \pm 1 \%, p<0.05$ ) (FIGURE 4B). Similarly, the frequency of IL17+ $\gamma \delta$ T cells declined. LAQ treatment reduced the proportion of IL- $17+\gamma \delta$ T cells from a mean of $16.6 \% \pm 2.7 \%$ in controls to a mean of $5.5 \% \pm 1.0 \%$ $(p<0.01)$ (FIGURE 4D).

The next step further investigated effects of LAQ on IL-17 production by measuring IL-17 secretion over time in a recall assay. For this purpose, lymph node cells from LAQ- or vehicletreated immunized mice were re-stimulated with $\mathrm{MOG}_{35-55}$ ex vivo. IL-17 concentrations in the supernatant were determined by ELISA.

The supernatant of lymph node cells, derived from vehicle-treated mice contained IL-17 at a mean concentration of $1437 \mathrm{pg} / \mathrm{ml} \pm 429 \mathrm{pg} / \mathrm{ml}$, whereas IL-17 was below the detection limit in the supernatant of all lymph node samples, isolated from LAQ-treated mice. This difference between LAQ and control group was highly significant with a p-value $<0.001$ (FIGURE 4E).

\subsubsection{LAQ reduced IFN -producing CD4+ T cells}

Effects of LAQ on the frequency of IFNY-producing CD4+ T cells (Th1 cells) were investigated by ex vivo stimulation of splenocytes isolated from LAQ-treated and control animals with PMA and ionomycin. Cytokine secretion was blocked and intracellular IFNY staining was analyzed by flow cytometry (FIGURE 5A).

LAQ significantly reduced the frequency of Th1 cells upon ex vivo stimulation from a mean of $2.6 \% \pm 0.9 \%$ in controls to a mean of $0.7 \% \pm 0.1 \%$ in LAQ-treated samples ( $p<0.05$ ) (FIGURE 5B). 
A

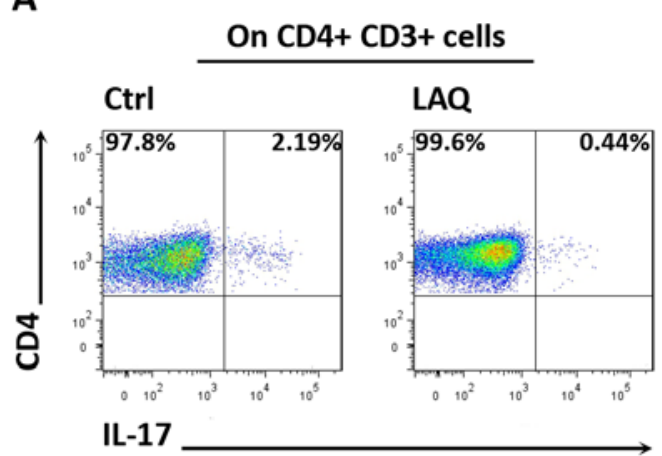

C

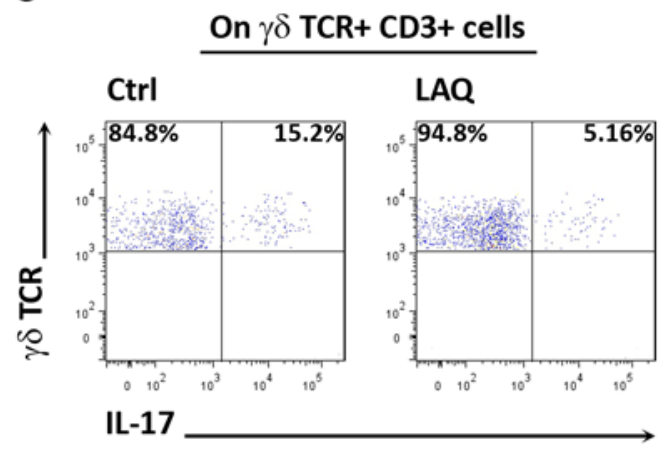

B

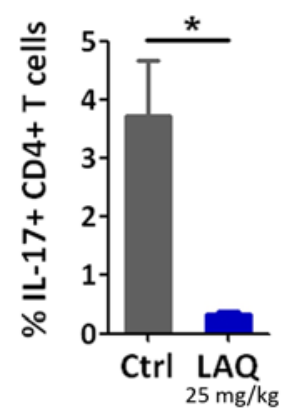

D

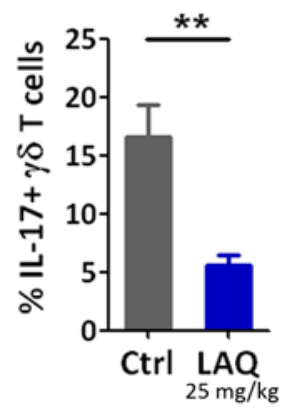

E

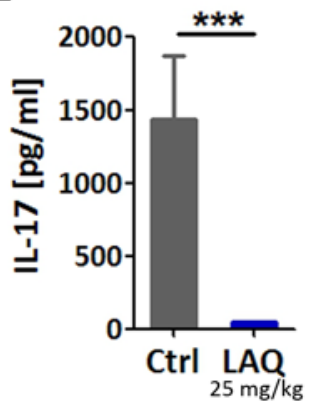

FIGURE 4: In vivo LAQ treatment reduces Th17 cells and IL-17-producing $\gamma \delta$ T cells

IL-17 production was analyzed in splenocytes and lymph node cells isolated from animals with prior $\mathrm{MOG}_{35-55}$-immunization and treatment with $25 \mathrm{mg} / \mathrm{kg}$ LAQ (blue) or vehicle (gray) for eleven days. The frequencies of IL-17+CD4+T cells $(\mathbf{A}, \mathbf{B})$ and IL-17+ $\gamma \delta$ T cells $(\mathbf{C}, \mathrm{D})$ upon ex vivo PMA and ionomycin stimulation were analyzed by flow cytometry. LAQ treatment significantly reduces the frequency of Th17 cells in the spleen ( $n=6$ per group, ${ }^{*} p<0.05$, unpaired t-test) (B). The frequency of IL-17-producing $\gamma \delta \mathrm{T}$ cells in the spleen is also reduced by LAQ therapy ( $n=6$ per group, $* *$ p $<0.01$, unpaired t-test) (D). Lymph node cells were restimulated with $\mathrm{MOG}_{35-55}$ ex vivo and cytokine secretion was assessed by ELISA. IL-17 levels are undetectable in the supernatants of in vivo LAQ-treated lymph node cells, whereas considerable amounts of IL-17 are measured in samples derived from vehicle-treated control animals (Ctrl: $n=11$, LAQ: $n=9, * * * p<0.001$, MannWhitney $U$ test) (E). Data are presented as mean \pm SEM. 
Furthermore, IFN $p$ secretion was determined in a recall assay. After re-stimulation of lymph node cells derived from LAQ- and vehicle-treated immunized animals with $\mathrm{MOG}_{35-55}$ ex vivo, IFN $\gamma$ concentrations were measured in the supernatants by ELISA. No IFN $\gamma$ was detected in the supernatant of any sample with in vivo LAQ treatment. In contrast, control samples produced a mean IFN $\gamma$ concentration of $836.6 \mathrm{pg} / \mathrm{ml} \pm 337.5 \mathrm{pg} / \mathrm{ml}$. This difference between LAQ and control group was highly significant with a p-value <0.001 (FIGURE 5C).

A

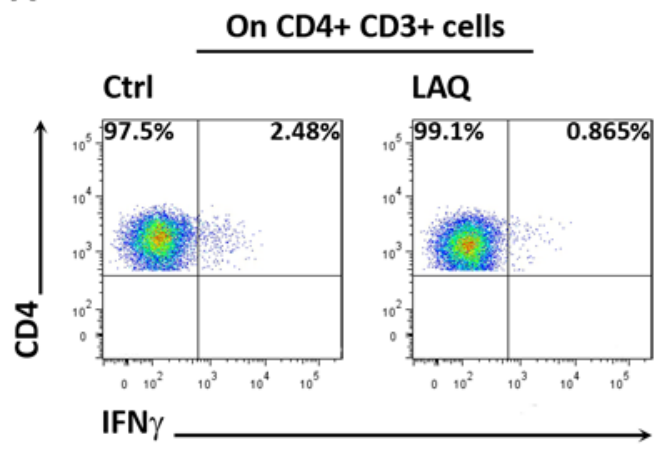

C

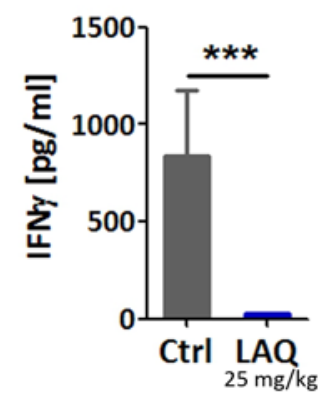

B

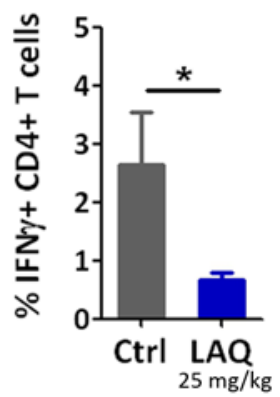

FIGURE 5: LAQ therapy reduces Th1 cells

IFN $\gamma$ production was evaluated in splenocytes and lymph node cells isolated from animals with prior $\mathrm{MOG}_{35-55}$-immunization and treatment with $25 \mathrm{mg} / \mathrm{kg}$ LAQ (blue) or vehicle (gray) for eleven days. Flow cytometry was used to analyze the frequency of IFN ${ }^{+}$ CD4+ T cells in the spleen upon ex vivo PMA and ionomycin stimulation (A, B). Flow cytometric data show a significant reduction of Th1 frequency after LAQ treatment ( $n=6$ per group,

* $p<0.05$, Mann-Whitney $U$ test) (B). IFN $\gamma$ secretion in lymph node cells was analyzed by ELISA after in vitro restimulation with $\mathrm{MOG}_{35-55}$. Quantitative data reveal a significantly lower IFNY secretion in lymph node cells from LAQ-treated animals compared to controls. IFN $\gamma$ concentrations in the supernatant of LAQtreated samples are under the detection limit of the assay, whereas control samples produce considerable amounts of IFNy (Ctrl: $n=11$, LAQ: $n=9, * * * p<0.001$, Mann-Whitney $U$ test) (C). Data are presented as mean \pm SEM.

\subsubsection{LAQ treatment increased the frequency of FoxP3+ regulatory $\mathrm{T}$ cells}

To analyze the effect of LAQ on anti-inflammatory $T$ cells, the frequency of regulatory T cells among splenic $C D 4+T$ cells was determined in naïve $\mathrm{C} 57 \mathrm{BI} / 6 \mathrm{~J}$ mice or $\mathrm{MOG}_{35-55}$-immunized mice treated with either $25 \mathrm{mg} / \mathrm{kg}$ LAQ or vehicle for 20 (naïve) or eleven days (immunized). Regulatory $\mathrm{T}$ cells are characterized by the expression of the lineage specific transcription factor 
FoxP3 (Hori et al., 2003). Thus, intracellular staining of FoxP3 was performed and analyzed by flow cytometry (FIGURE 6A).

Data analysis revealed a LAQ-induced increase in the frequency of regulatory $T$ cells compared to controls. Furthermore, this effect was independent of a prior immunization with $M O_{35-55}$. In immunized mice, LAQ led to a significant rise of regulatory $T$ cells from a mean frequency of $9.3 \% \pm 1.1 \%$ in controls to $14.7 \% \pm 0.8 \%$ in treated animals ( $p<0.01$ ) (FIGURE 6B). In naïve mice, LAQ treatment induced a similar increase of the mean frequency of regulatory $T$ cells from $11.4 \% \pm 0.3 \%$ in controls to $14.9 \% \pm 0.6 \%$ in treated animals ( $p<0.001$ ) (FIGURE $6 C$ ).

A

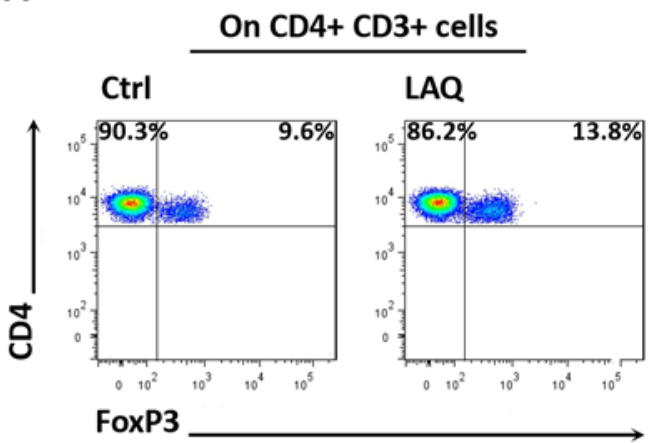

B

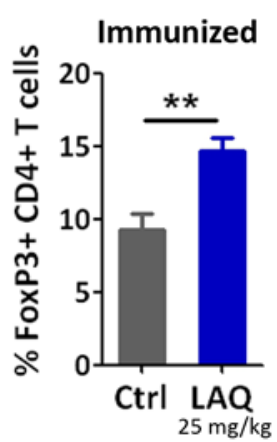

C

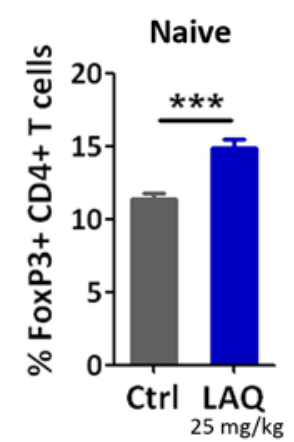

FIGURE 6: Regulatory T cells are increased in naïve and immunized mice after LAQ treatment

The frequency of FoxP3+ regulatory $T$ cells was analyzed in splenocytes from $\mathrm{MOG}_{35-55}$-immunized and naïve animals which had received treatment with $25 \mathrm{mg} / \mathrm{kg}$ LAQ (blue) or vehicle (gray) for eleven (immunized) or 20 days (naïve) by flow cytometry (A). LAQ treatment significantly augments the frequency of FoxP3+ regulatory T cells in immunized ( $n=6$ per group, ${ }^{* *} p<0.01$, Mann-Whitney $U$ test) (B) and in naïve mice ( $n=5$ per group, ${ }^{* * *} p<0.001$, unpaired t-test) (C). Data are presented as mean \pm SEM.

\subsubsection{LAQ had no direct effect on IL-17+ and IFN + + CD4+ T cells in vitro}

To investigate whether T cells are a direct target of LAQ therapy, splenocytes were isolated from animals immunized with $\mathrm{MOG}_{35-55}$ which had not received prior LAQ treatment in vivo and were incubated in vitro with 0 or $5 \mu \mathrm{M}$ LAQ for $72 \mathrm{~h}$. The cells were subsequently stimulated with PMA and ionomycin and the frequency of IL-17+ and IFN $\mathrm{P}+\mathrm{CD} 4+\mathrm{T}$ cells was analyzed by flow cytometry (FIGURE 7A\&C).

In vitro treatment with $5 \mu \mathrm{M}$ LAQ did not significantly alter the frequencies of Th17 and Th1 cells. The mean frequency of Th17 cells after a $72 \mathrm{~h}$ incubation period was very low in the control group (mean $1.1 \% \pm 1 \%$ ) as well as in the LAQ-treated group (mean $0.6 \% \pm 0.1 \%, p>0.05$ ) 
(FIGURE 7B). The frequency of Th1 cells after $72 \mathrm{~h}$ of incubation was similar to the frequency obtained after direct ex vivo stimulation (compare 3.1.3.2 and FIGURE 5B), but no difference was detected between the control (mean 3.8\% $\pm 0.5 \%$ ) and the LAQ-treated group (mean $3.7 \% \pm 0.6 \%$, $p<0.05)$ (FIGURE 7D).

A

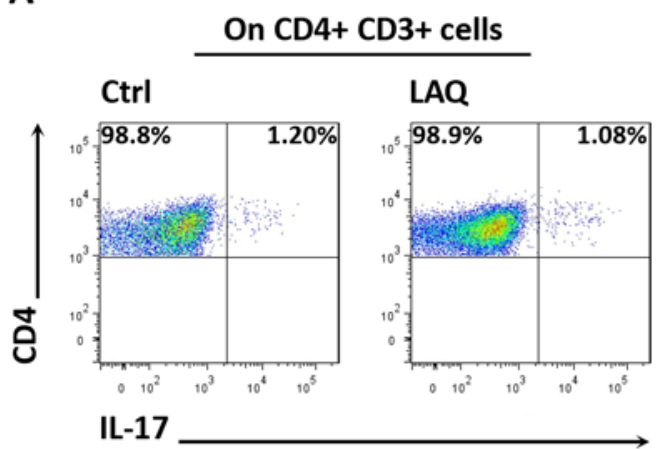

C

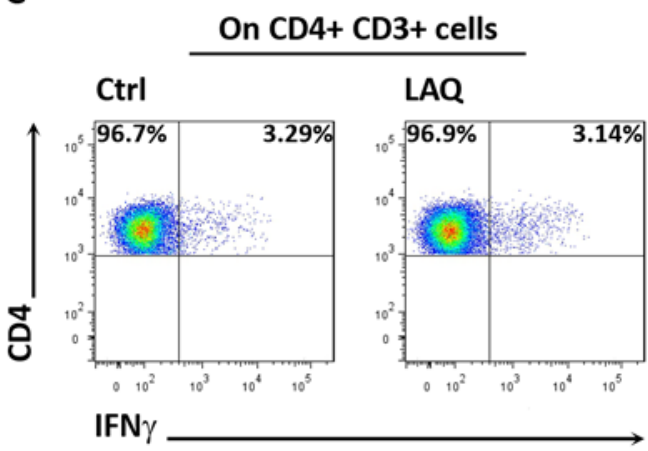

B

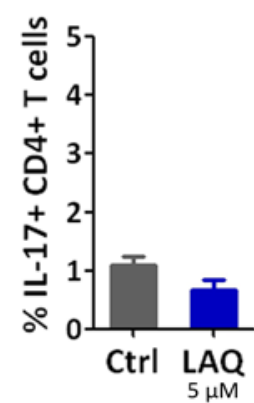

D

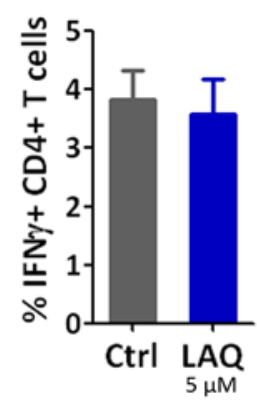

FIGURE 7: LAQ has no direct effect on Th17 and Th1 cells in vitro In vitro effects of LAQ treatment on pro-inflammatory $T$ cell subsets, isolated from the spleen of immunized mice, were investigated using flow cytometry. Frequencies of IL-17+ (A, B) and IFNp+ (C, D) CD4+ T cells were measured after in vitro treatment with 0 (gray) or $5 \mu \mathrm{M}$ LAQ (blue) for $72 \mathrm{~h}$ and subsequent PMA and ionomycin stimulation for $5 \mathrm{~h}$. LAQ does not significantly alter the frequencies of Th17 (B) and Th1 (D) cells in vitro ( $\mathrm{n}=6$ per group, $p>0.05$, Mann-Whitney $U$ test). Data are presented as mean \pm SEM.

\subsection{Preventive LAQ treatment decreased total dendritic cells and myeloid dendritic cells in vivo}

To investigate the effects of LAQ treatment on antigen presenting cells, LAQ-induced changes were evaluated in splenic dendritic cells. The frequencies of total dendritic cells and myeloid dendritic cells in the spleen served as readouts for the therapeutic effects of $L A Q$.

Naïve $\mathrm{C} 57 \mathrm{BI} / 6 \mathrm{~J}$ mice or $\mathrm{MOG}_{35-55}$-immunized mice received daily oral treatment with either $25 \mathrm{mg} / \mathrm{kg}$ LAQ or vehicle for 20 (naïve) or eleven days (immunized). Splenocytes were isolated and analyzed ex vivo by flow cytometry. 


\subsubsection{LAQ treatment reduced the frequency of dendritic cells}

The effect of LAQ on splenic dendritic cells was first evaluated by analyzing the frequency of total dendritic cells in the spleen of immunized or naïve animals, which had received either 25 $\mathrm{mg} / \mathrm{kg}$ LAQ or vehicle. Dendritic cells were defined as MHCII+CD11 $\mathrm{c}^{\text {high }}$ cells in the flow cytometric analysis (FIGURE 8A).

The frequencies of splenic dendritic cells were considerably reduced after LAQ treatment and this effect was independent of the immunization with $\mathrm{MOG}_{35-55}$ - as evidenced by parallel investigations in naïve mice. In immunized mice, the mean frequency of dendritic cells decreased from $1.6 \% \pm 0.2 \%$ in control animals to $0.6 \% \pm 0.3 \%$ in LAQ-treated animals $(p<0.01)$ (FIGURE 8B). In naïve mice, the frequency of dendritic cells in the spleen was slightly lower, but still a significant reduction was detected when comparing control animals (mean $0.8 \% \pm 0.1 \%$ ) to LAQ-treated animals (mean $0.4 \% \pm 0.02 \%, \mathrm{p}<0.01$ ) (FIGURE 8 C).

A

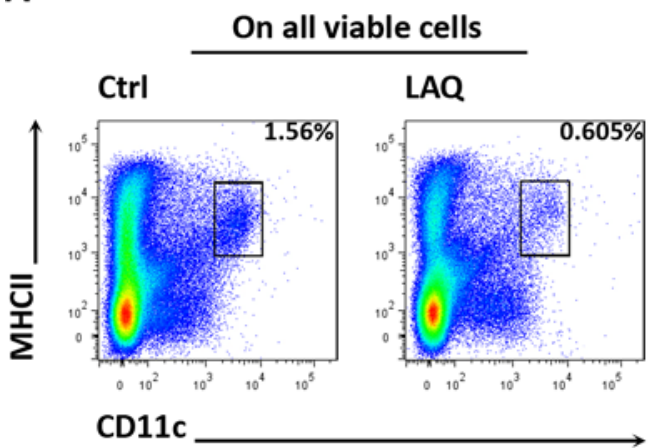

B

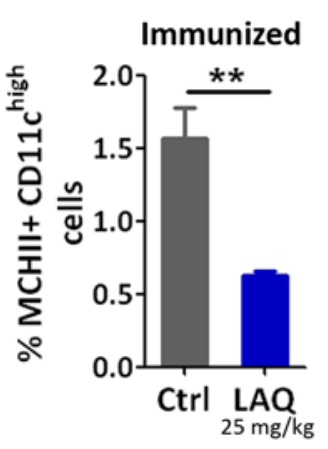

C

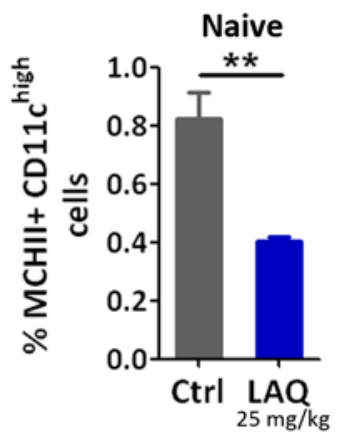

FIGURE 8: LAQ decreases the frequency of dendritic cells in the spleen

Splenocytes were isolated from $\mathrm{MOG}_{35-55}$-immunized and naïve animals which had received treatment with $25 \mathrm{mg} / \mathrm{kg}$ LAQ (blue) or vehicle (gray) for eleven (immunized) or 20 days (naïve) in vivo. Dendritic cell frequencies were investigated by flow cytometry (A). LAQ treatment significantly decreases dendritic cell frequencies independently of a prior immunization. LAQ approximately halves the proportion of dendritic cells compared to controls in immunized ( $n=6$ per group, ${ }^{* *} p<0.01$, unpaired t-test) (B) and naïve animals (Ctrl: $n=7$, LAQ: $n=6,{ }^{*} p<0.05$, unpaired t-test) (C). Data are presented as mean \pm SEM. 


\subsubsection{LAQ treatment decreased myeloid dendritic cells}

Effects of LAQ on dendritic cells were further characterized by assessing the frequency of myeloid dendritic cells in the spleen of naïve and immunized mice with or without prior LAQ treatment $(25 \mathrm{mg} / \mathrm{kg})$. Myeloid dendritic cells are characterized by the expression of the surface marker CD11b. The frequency of CD11b+ myeloid dendritic cells among total dendritic cells was analyzed using flow cytometry (FIGURE 9A).

LAQ treatment significantly decreased the frequency of $C D 11 b+$ myeloid dendritic cells in the spleen of immunized as well as naïve mice. In immunized animals, LAQ diminished the mean frequency of myeloid dendritic cells of about $5 \%$, from $80.5 \% \pm 1.8 \%$ in controls to $75.6 \% \pm 0.6 \%$ in treated animals $(p<0.05)$ (FIGURE 9B). In naïve mice, the LAQ-induced decrease in the mean frequency of myeloid dendritic cells was even more pronounced. LAQ treatment reduced the mean frequency of about $21 \%$, from $74.2 \% \pm 2.3 \%$ in controls to $53.1 \% \pm 1.8 \%$ in treated animals $(p<0.001)$ (FIGURE 9C).

A

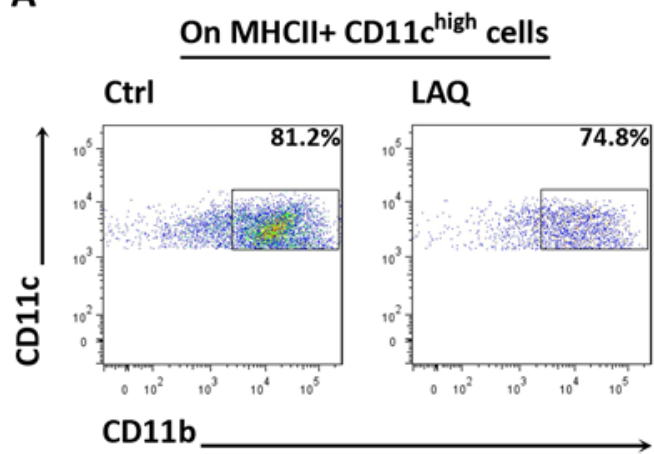

B

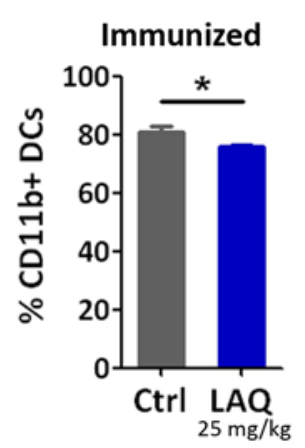

C

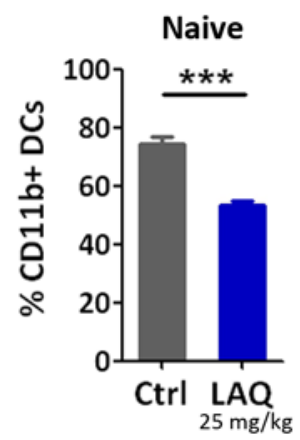

\section{FIGURE 9: LAQ reduces myeloid dendritic cells in the spleen}

Myeloid dendritic cells were investigated in spleens from MOG $_{35-55}$-immunized and naïve animals which had received treatment with $25 \mathrm{mg} / \mathrm{kg}$ LAQ (blue) or vehicle (gray) for eleven (immunized) or 20 days (naïve) in vivo. The proportion of $\mathrm{CD} 11 \mathrm{~b}+$ myeloid dendritic cells among all dendritic cells was analyzed ex vivo by flow cytometry (A). LAQ treatment significantly reduces the frequencies of CD11b+ myeloid dendritic cells in immunized as well as in naïve mice. In immunized mice myeloid dendritic cells are decreased by about $5 \%$ after LAQ treatment ( $n=6$ per group, ${ }^{*} \mathrm{p}<0.05$, unpaired $\mathrm{t}$-test) (B). In naïve mice $\mathrm{LAQ}$ leads to a reduction of 21\% (Ctrl: $n=7$, LAQ: $n=6, * * * p<0.001$, unpaired t-test) (C). Data are presented as mean \pm SEM. 


\subsection{Preventive LAQ treatment led to the activation of natural killer cells in vivo}

To further study effects of LAQ on innate immunity, the next set of experiment investigated LAQ-induced alterations in NK cell numbers, subpopulations, activation state and effector functions. Therefore, naïve and $\mathrm{MOG}_{35-55}$-immunized mice received daily oral treatment with either $25 \mathrm{mg} / \mathrm{kg}$ LAQ or vehicle for 20 (naïve) or eleven days (immunized). Splenocytes were isolated from these mice and analyzed ex vivo.

\subsubsection{LAQ treatment reduced absolute NK cell numbers, but did not alter NK cell frequencies}

To analyze absolute NK cell numbers and NK cell frequency by flow cytometry, the NK cell specific surface marker NK1.1 was used (FIGURE 10D). NK1.1 is expressed on virtually all NK cells in $\mathrm{C} 57 \mathrm{BI} / 6 \mathrm{~J}$ mice and can be used as pan-NK cell marker. Besides NK cells a small subset of CD3+ T cells presents NK1.1 at the cell surface. These cells, denominated NKT cells, respond to lipidantigens and display immunoregulatory properties (Godfrey et al., 2010). The current study excluded NKT cells from the analysis by pre-gating on CD19- CD3-cells.

The impact of LAQ treatment on NK cell numbers was assessed by extrapolating NK cell counts from flow cytometric samples to total splenocyte numbers determined in a Neubauer chamber. Analysis of total splenocyte numbers revealed a $50 \%$ decrease in immunized LAQtreated mice (mean $40.8 * 10^{6} \pm 6.9 * 10^{6}$ ) compared to controls (mean $84.8^{*} 10^{6} \pm 12.1 * 10^{6}$, p<0.01) (FIGURE 10A). Absolute NK cell numbers were also reduced by approximately $50 \%$ in immunized LAQ-treated mice (mean $0.7 * 10^{6} \pm 0.2 * 10^{6}$ ) compared to controls (mean $\left.1.7 * 10^{6} \pm 0.4 * 10^{6}, p=0.07\right)$ (FIGURE 10B). The Spearman correlation analysis of the total numbers of splenocytes and the absolute NK cell numbers revealed a very significant correlation $(p<0.01$; $\left.r_{s}=0.84\right)$ (FIGURE 10C).

NK cell frequency was calculated from flow cytometric data as percent NK1.1+ cells of all CD19- CD3- splenocytes (FIGURE 10D). The analysis of NK cell frequencies in the spleen of immunized mice showed a similar proportion of NK cells in LAQ-treated (mean $5.8 \% \pm 0.7 \%$ ) and control mice (mean $4.5 \% \pm 0.8 \%, p>0.05$ ) (FIGURE 10E). 
A

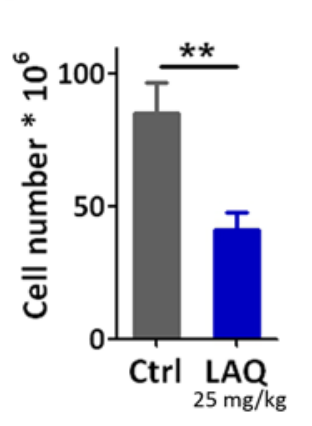

D

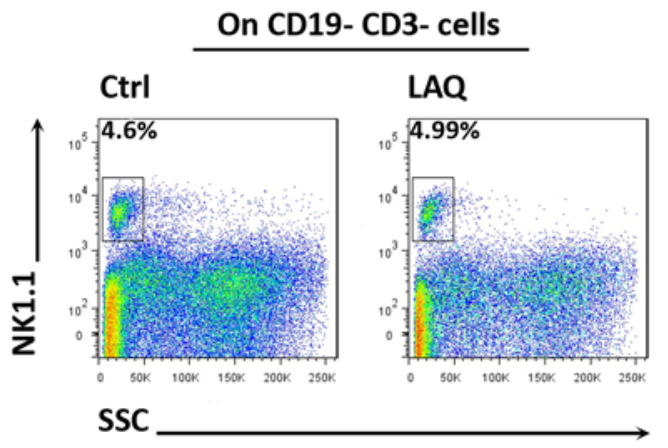

C

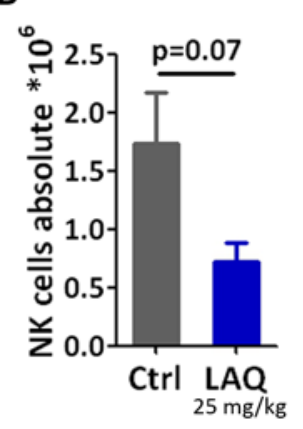

E

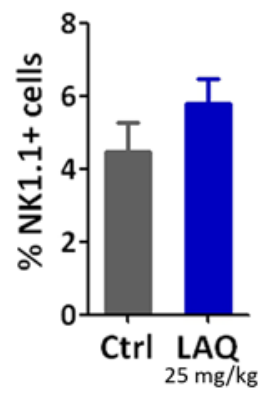

\section{FIGURE 10: LAQ reduces absolute NK cell numbers, but does not alter NK cell frequencies}

Total splenocyte numbers, absolute NK cell numbers and NK cell frequencies were assessed in mice, immunized with $\mathrm{MOG}_{35-55}$ and treated with LAQ (25 mg/kg) (blue) or vehicle (gray) for eleven days in vivo. The total numbers of splenocytes were counted using a Neubauer chamber. Frequencies of NK1.1+ NK cells were analyzed by flow cytometry (D, E) and absolute NK cell numbers were extrapolated from these data. LAQ treatment halves the total number of splenocytes ( $n=6$ per group, ${ }^{* *} p<0.01$, unpaired t-test) (A). A similar LAQ-induced decrease is observed for absolute NK cell numbers ( $n=6$ per group, $p=0.07$, unpaired $t$ test) (B). Spearman correlation analysis reveals a significant correlation between total splenocyte and absolute NK cell numbers $\left({ }^{* *} p<0.01, r_{s}=0.84\right)$ (C). NK cell frequencies among all CD19- CD3- cells are not significantly altered upon LAQ treatment ( $n=6$ per group, $p>0.05$, unpaired t test) (E). Data are presented as mean \pm SEM. 


\subsubsection{LAQ increased activation markers on the surface of NK cells}

Effects of LAQ on the phenotype of NK cells were evaluated by investigating NK cell molecules which reflect their subpopulations and activation state.

\subsubsection{LAQ treatment shifted NK cell subsets towards more CD27+ NK cells}

The different subpopulations of murine NK cells are characterized by a distinct expression pattern of the surface molecules CD27 and CD11b. Using flow cytometry, CD27+ CD11b- NK cells can be distinguished from CD27+CD11b+ NK cells and CD27- CD11b+ NK cells (Chiossone et al., 2009). CD27+CD11b- and CD27- CD11b+ NK cells will be further referred to as CD27+ and CD11b+ NK cells, respectively, in this work.

To investigate effects of LAQ on NK cell subpopulations, the proportions of CD27+, CD27+ CD11b+ and CD11b+ NK cells were analyzed within the population of NK1.1+ CD3- cells by flow cytometry using the classification mentioned above (FIGURE 11A).

LAQ treatment profoundly altered NK cell subpopulations in naïve as well as in immunized mice. LAQ induced a shift towards more CD27+ and fewer CD11b+ NK cells, whereas the double positive cells remained constant. In immunized mice, CD27+ NK cells were increased by about $18 \%$ in LAQ-treated animals (mean $36.8 \% \pm 2.3 \%$ ) compared to controls (mean $18.8 \% \pm 3.9 \%$, $p<0.01$ ). CD11b+ NK cells were decreased by about $20 \%$ in treated animals (mean $34.0 \% \pm 2.4 \%$ ) compared to controls (mean $55.9 \% \pm 3.6 \%, p<0.001$ ). Double positive NK cells showed similar frequencies in LAQ-treated (mean $22.7 \% \pm 1.2 \%$ ) and control animals (mean $19.3 \% \pm 1.7 \%$, p >0.05) (FIGURE 11B).

Data from naïve mice revealed a similar LAQ effect on NK cell subpopulations, but in general these animals displayed a higher frequency of CD11b+ and a lower frequency of CD27+ NK cells in both treatment groups compared to immunized mice. LAQ treatment increased the mean proportion of $\mathrm{CD} 27+\mathrm{NK}$ cells from $8.6 \% \pm 1.7 \%$ in control animals to $22.4 \% \pm 3.6 \%$ in treated animals $(p<0.01)$. CD11b+NK cells were decreased upon LAQ therapy from $70.4 \% \pm 1.8 \%$ in controls to $57.5 \% \pm 4.2 \%$ in treated animals $(p<0.05)$. As in immunized animals, LAQ did not influence the frequency of double positive NK cells in naive mice (Ctrl: mean $18.4 \% \pm 1.0 \%$; LAQ: mean $16.9 \% \pm 1.1 \% ; p>0.05)$ (FIGURE 11C). 
A

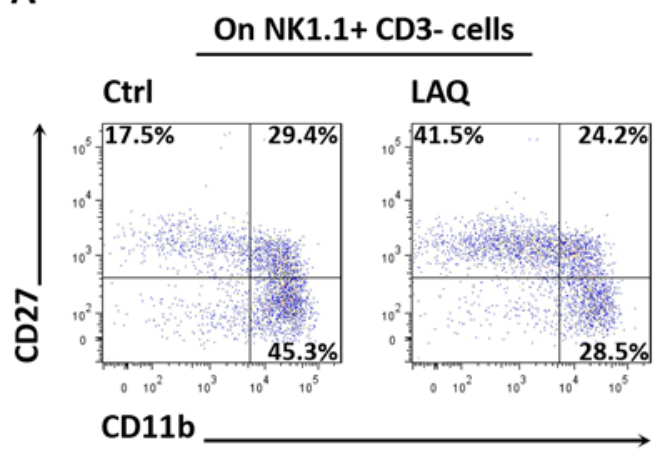

B Immunized
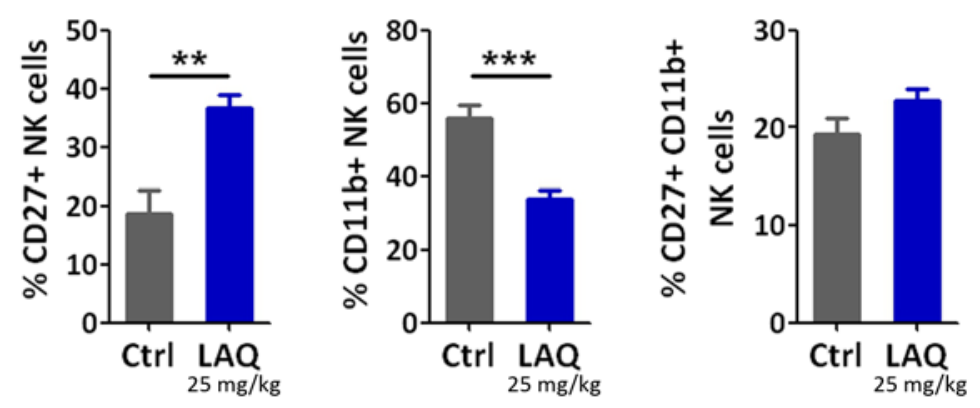

C Naive
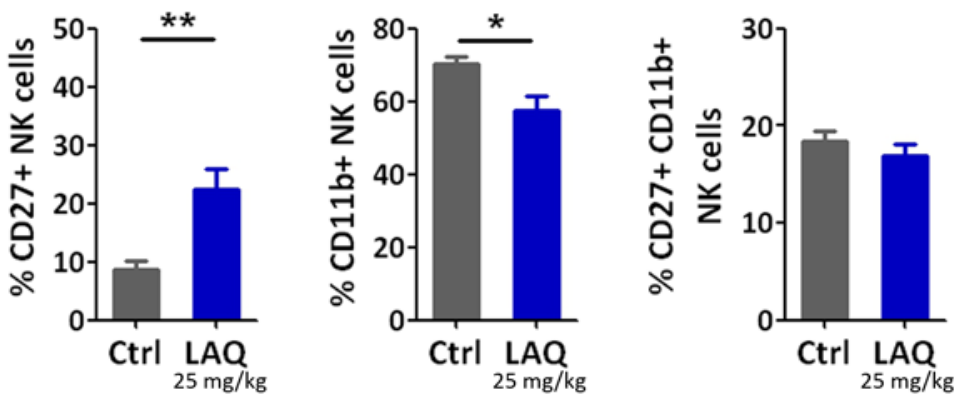

FIGURE 11: LAQ interferes with the relative frequency of NK cell subpopulations

NK cell subpopulations were investigated in the spleen of $M_{35}$-55-immunized and naive animals which had received treatment with 25 $\mathrm{mg} / \mathrm{kg}$ LAQ (blue) or vehicle (gray) for eleven (immunized) or 20 days (naïve) in vivo. The expression of CD27 and CD11b on the surface of NK1.1+ cells was analyzed by flow cytometry (A). Subsets of NK cells are classified as follows: CD27+ CD11b- (CD27+), CD27+ CD11b+ (double positive) and CD27- CD11b+ (CD11b+). LAQ treatment induces a shift in NK cell subpopulations towards more CD27+ and fewer CD11b+ cells. In immunized animals LAQ increases CD27+ NK cells of about 20\% (Ctrl: $n=8, L A Q: n=9, * * p<0.01$, Mann-Whitney $U$ test) and reciprocally decreases CD11b+NK cells ( ${ }^{* * *} \mathrm{p}<0.01$, unpaired t-test) (B). LAQ exerts similar effects in naïve mice, where an increase of about $14 \%$ is observed for CD27+ NK cells ( $n=7$ per group, ${ }^{* *} p<0.01$, unpaired $t$-test) and a decrease of $13 \%$ for CD11b+ NK cells ( ${ }^{*} p<0.05$, unpaired t-test) $(C)$. The proportion of CD27+CD11b+ NK cells remains stable under LAQ treatment in immunized (Ctrl: $n=8, L A Q: n=9, p>0.05$, unpaired t-test) (B) and naïve mice $(n=7$ per group, $p>0.05$, unpaired t-test) (C). Data are presented as mean \pm SEM.

\subsubsection{LAQ treatment enhanced NK cell activation}

To evaluate the impact of LAQ on the activation state of NK cells, the next set of experiments investigated the expression of the early activation antigen CD69 as well as a panel of activating and inhibitory NK cell receptors. 


\subsection{LAQ increased the frequency of CD69+ NK cells}

The surface molecule CD69 is expressed on T cells and NK cells shortly after activation (Lanier et al., 1988, Werfel et al., 1997). Therefore, CD69 is widely used as an activation marker for these cells.

To analyze the effect of LAQ on NK cell activation, the frequency of CD69+ NK cells among all NK1.1+ CD3- cells was measured by flow cytometry (FIGURE 12A). Splenocytes were isolated from naïve and immunized mice with or without prior LAQ treatment $(25 \mathrm{mg} / \mathrm{kg})$ in vivo.

LAQ treatment markedly increased the frequency of CD69+ NK cells among all NK1.1+ CD3- cells in naïve and immunized mice. Immunized control animals displayed a mean frequency of CD69+ NK cells of $40.4 \% \pm 5.7 \%$, which was nearly doubled in LAQ-treated mice to $76.0 \% \pm$ $3.0 \%$ ( $p<0.001$ ) (FIGURE 12B). In naïve mice, LAQ increased the mean frequency of CD69+ NK cells from $54.6 \% \pm 4.8 \%$ in controls to $80.5 \% \pm 3.5 \%$ in treated animals $(p<0.001$ ), which corresponds to a relative increase of approximately 50\% (FIGURE 12C).

Further analyses investigated whether the LAQ-induced increase in CD69 expression was restricted to a distinct subpopulation of NK cells. For this purpose, CD69 expression was evaluated on CD27+ NK cells and CD11b+ NK cells from spleens of immunized mice with and without prior LAQ treatment $(25 \mathrm{mg} / \mathrm{kg})$.

Both subpopulations responded to in vivo LAQ treatment with considerable upregulation of $C D 69$. In the subpopulation of $C D 27+$ NK cells LAQ approximately doubled the mean frequency of CD69+ NK cells from $29.4 \% \pm 4.2 \%$ in controls to $67.3 \% \pm 5.5 \%$ in treated animals $(p<0.001)$ (FIGURE 12D). In the subpopulation of CD11b+ NK cells LAQ induced a 3.9 fold increase of CD69+ cells from $3.6 \% \pm 0.4 \%$ in controls to $13.9 \% \pm 0.9 \%$ in treated animals ( $p<0.001$ ) (FIGURE 12E). In summary the relative increase of CD69+ cells after LAQ treatment was more pronounced in CD11b+ NK cells, but the absolute frequency of activated cells was higher in CD27+ NK cells. Thus, the LAQ-mediated upregulation of CD69 was not restricted to a specific subpopulation of NK cells. 
A

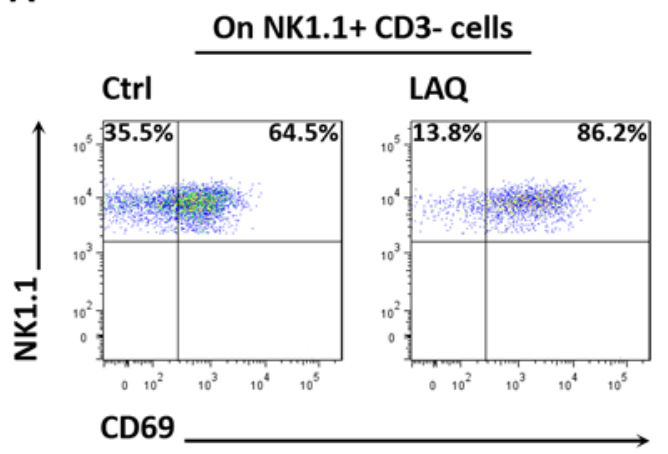

B

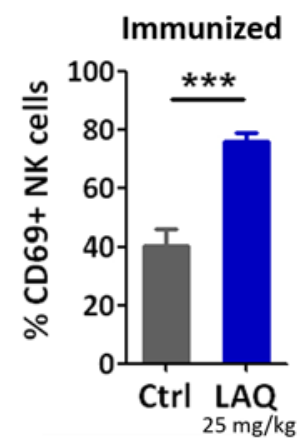

D

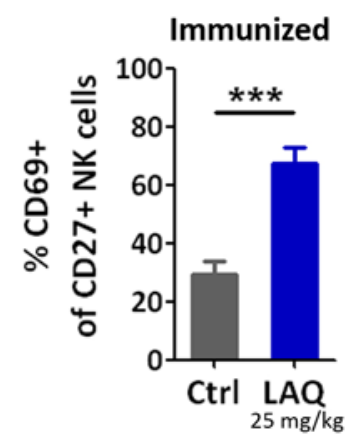

C

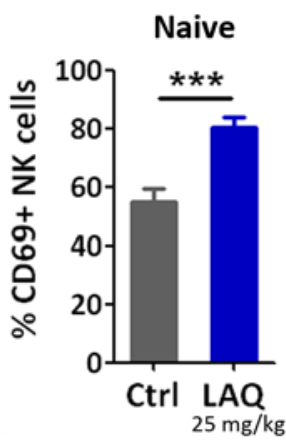

E

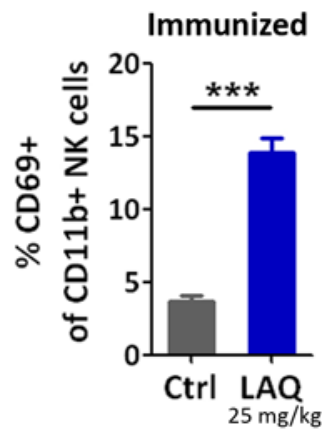

FIGURE 12: LAQ augments the proportion of CD69+ NK cells

To investigate the effects of $L A Q$ on the activation of NK cells, splenocytes were isolated from $M_{35-55}$-immunized and naïve animals which had received treatment with $25 \mathrm{mg} / \mathrm{kg}$ LAQ (blue) or vehicle (gray) for eleven (immunized) or 20 days (naïve) in vivo. The proportion of CD69+ cells among all NK1.1+ NK cells, CD27+ NK cells and CD11b+ NK cells was analyzed by flow cytometry (A). LAQ treatment nearly doubles the frequency of CD69+ NK cells in immunized animals ( $n=11$ per group, ${ }^{* * *} \mathrm{p}<0.001$, unpaired t-test) (B). In naïve animals, LAQ also induces a $50 \%$ increase of CD69+ NK cells relative to controls ( $n=9$ per group, *** $\mathrm{p}<0.001$, Mann-Whitney $\mathrm{U}$ test) (C). LAQ raises the proportion of $\mathrm{CD} 69+\mathrm{NK}$ cells among $\mathrm{CD} 27+\mathrm{NK}$ cells ( $\mathrm{n}=10$ per group, ${ }^{* * *} \mathrm{p}<0.001$, unpaired $\mathrm{t}$-test) (D) as well as CD11b+ NK cells ( $n=10$ per group, *** $\mathrm{p}<0.001$, unpaired $\mathrm{t}$-test) $(\mathrm{E})$. Data are presented as mean \pm SEM.

\subsection{LAQ upregulated activating NK cell receptors}

The interplay of different activating and inhibitory receptors controls the activation of NK cell effector functions (Long et al., 2013).

To further characterize LAQ-mediated changes on the activation state of NK cells, this thesis investigated the expression of different activating NK cell receptors by flow cytometry. Splenocytes were derived from $\mathrm{MOG}_{35-55}$-immunized animals which had received $25 \mathrm{mg} / \mathrm{kg} \mathrm{LAQ}$ or vehicle for eleven days in vivo. The data were analyzed as percentage of positive cells among NK1.1+ CD3- cells (FIGURE 13A, C, E \& G). The following activating NK cell receptors were investigated: TACTILE, DNAM-1, NKG2D, CD2, Ly49D, CD244.2 and LFA-1. 
LAQ treatment significantly increased the mean frequencies of TACTILE+, DNAM-1+ and NKG2D+ NK cells. The mean frequency of TACTILE+ NK cells was elevated from $71.5 \% \pm 1.5 \%$ in controls to $90.3 \% \pm 0.7 \%$ in treated animals $(p<0.001$ ) (FIGURE 13B). DNAM- $1+$ NK cells showed an increase of about $10 \%$ upon LAQ treatment (Ctrl: mean $59.4 \% \pm 2.5 \%$; LAQ: mean $70.9 \% \pm 2.4 \%$; $p<0.01$ ) (FIGURE 13D). The mean frequency of NKG2D+ NK cells was slightly, but significantly higher in LAQ-treated animals (mean $96.5 \% \pm 0.3 \%$ ) compared to controls (mean $92.6 \% \pm 0.9 \%$, p<0.001) (FIGURE 13F).

The only activating NK cell receptor of this panel that showed a decreased frequency after $L A Q$ treatment was $C D 2$. The mean frequency of $C D 2+N K$ cells was reduced from $99.4 \% \pm 0.2 \%$ in controls to $95.0 \% \pm 0.5 \%$ in treated animals $(p<0.001$ ) (FIGURE $13 \mathrm{H}$ ). LAQ had no influence on the frequencies of Ly49D+, CD244.2+ and LFA-1+ NK cells.

Additional analyses examined if the upregulated expression of TACTILE and DNAM-1 by LAQ was restricted to NK cells. Since T cells can express TACTILE and DNAM-1, their receptor expression was analyzed on CD3+ T cells (FIGURE 14A \& C). In contrast to NK cells, LAQ treatment did not lead to an upregulation of TACTILE and DNAM-1 on T cells. The proportion of TACTILE+ T cells was similar in animals with (mean $60.4 \% \pm 1.4 \%$ ) and without LAQ treatment (mean $62.5 \% \pm 1.8 \%, p>0.05$ ) (FIGURE 14B). DNAM-1 was clearly down-regulated on T cells after LAQ treatment (Ctrl: mean 49.1\% $\pm 1.6 \%$; LAQ: mean 44.3\% $\pm 0.8 \%$; $p<0.05$ ) (FIGURE 14C).

\subsection{LAQ downregulated inhibitory NK cell receptors}

Inhibitory NK cell receptors act in an antagonistic way to activating NK cell receptors in the regulation of NK cell effector functions and therefore play an important role in blocking or reducing NK cell activation (Long et al., 2013).

To investigate effects of LAQ on inhibitory NK cell receptors, splenocytes from mice immunized with $\mathrm{MOG}_{35-55}$, which had received prior treatment with either $25 \mathrm{mg} / \mathrm{kg}$ LAQ or vehicle in vivo, were analyzed for the expression of several inhibitory receptors on the surface of NK cells by flow cytometry. The data were analyzed as percentage of positive cells among NK1.1+ CD3- cells (FIGURE 15A \& C). The following inhibitory NK cell receptors were investigated: TIGIT, Ly49C/I/F/H, Ly49G2 and Ly49A.

LAQ treatment significantly reduced the frequencies of TIGIT+ and Ly49C/I/F/H+ NK cells. The mean frequency of TIGIT+ NK cells was decreased from $10.1 \% \pm 1.3 \%$ in controls to $3.9 \% \pm$ $0.6 \%$ in LAQ-treated animals ( $p<0.001$ ) (FIGURE 15B). Mean frequencies of Ly49C/I/F/H+ NK cells were diminished from $69.9 \% \pm 1.0 \%$ in controls to $64.0 \% \pm 1.3 \%$ in LAQ-treated animals $(p<0.01)$ (FIGURE 15D). The frequencies of Ly49G2+ and Ly49A+ NK cells were similar in LAQ- and vehicletreated animals. 
A

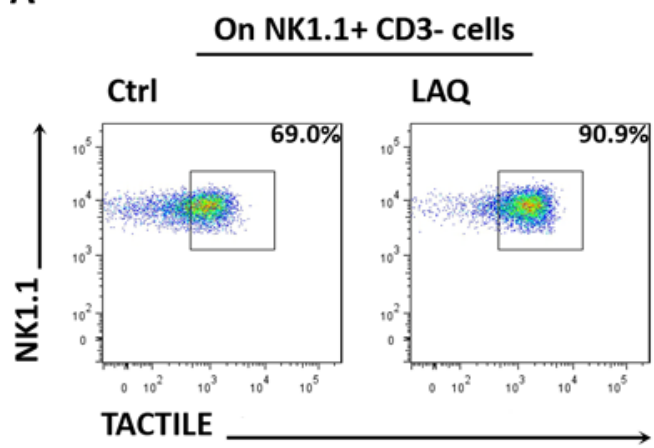

C

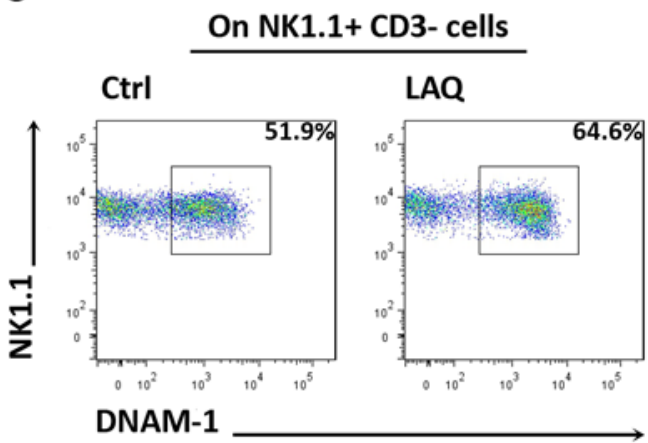

E

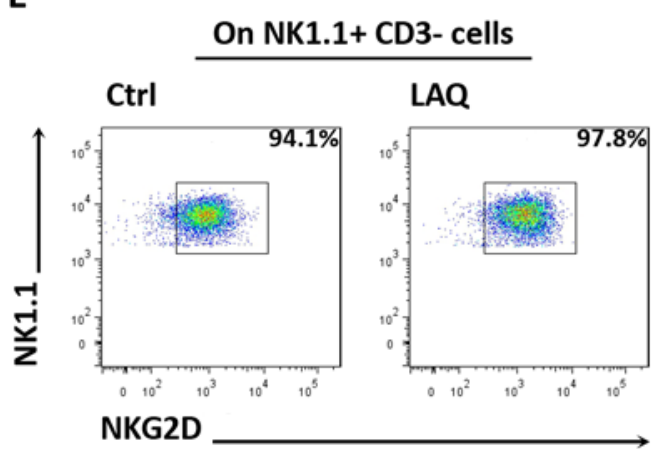

G

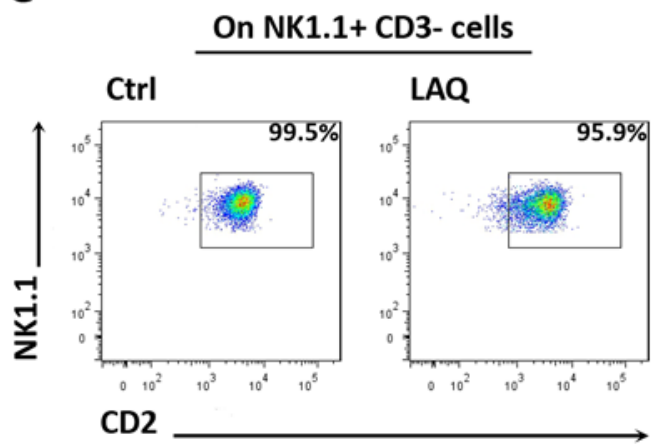

B

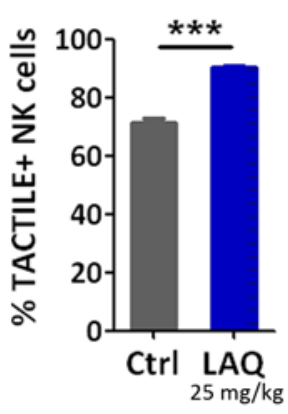

D

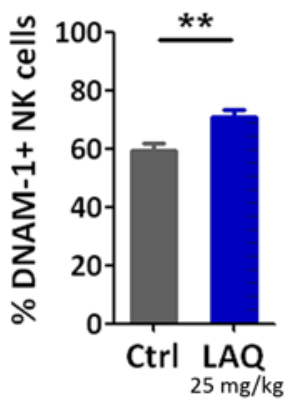

F

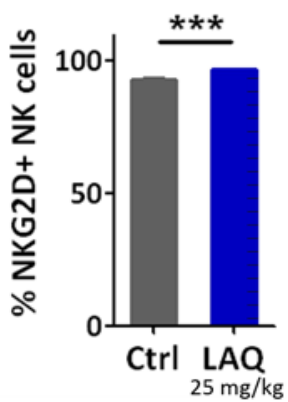

H

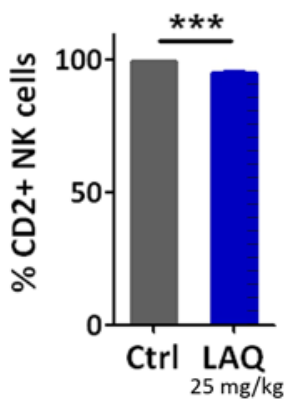

FIGURE 13: LAQ increases the expression of activating NK cell receptors

The effects of LAQ on activating NK cell receptors were assessed ex vivo in splenocytes from mice immunized with $M_{35}$-55 and treated with LAQ $(25 \mathrm{mg} / \mathrm{kg})$ (blue) or vehicle (gray) for eleven days in vivo. The surface expression of TACTILE (A, B), DNAM-1 (C, D), NKG2D (E, F) and $\mathrm{CD2}(\mathbf{G}, \mathbf{H})$ was analyzed by flow cytometry. LAQ treatment significantly raises the proportion of TACTILE+ NK cells $(n=10$ per group, $* * * \quad p<0.001$, unpaired t-test) (B), DNAM-1+ NK cells ( $n=10$ per group, ** $p<0.01$, unpaired t-test) (D) and NKG2D+ NK cells ( $n=10$ per group, ${ }^{* * *} \mathrm{p}<0.001$, MannWhitney $U$ test) (F). The frequency of CD2+ NK cells is slightly reduced upon LAQ treatment ( $n=10$ per group, *** $p<0.001$, Mann-Whitney U test) $(\mathbf{H})$. Data are presented as mean \pm SEM. 
A

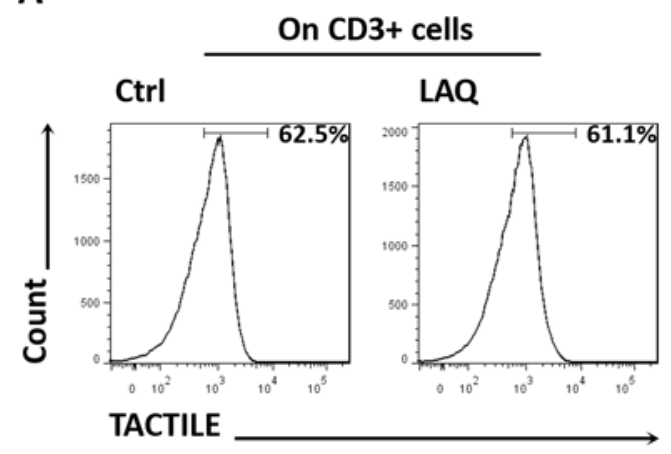

C

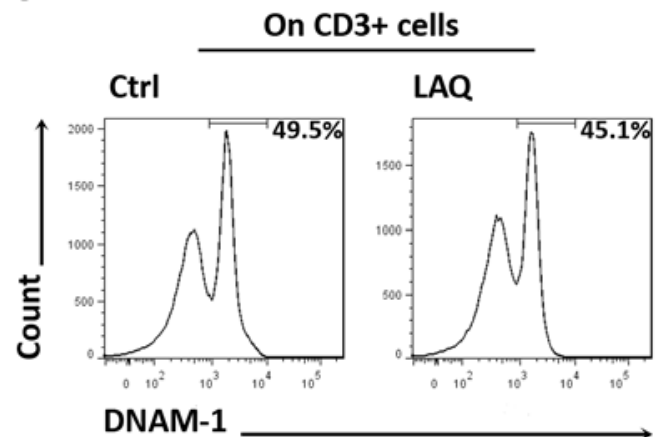

B

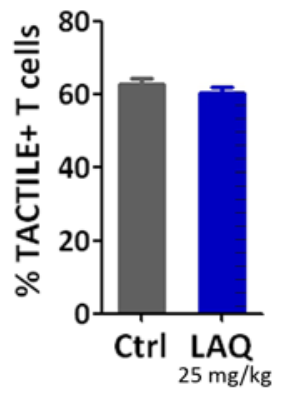

D

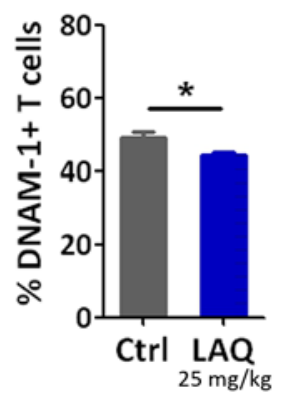

FIGURE 14: Effects of LAQ on the expression of TACTILE and DNAM-1 on T cells

The effects of LAQ on the expression of TACTILE and DNAM-1 on T cells were evaluated ex vivo in the spleens of mice immunized with $M O_{35-55}$ and treated with $L A Q(25 \mathrm{mg} / \mathrm{kg}$ ) (blue) or vehicle (gray) for eleven days in vivo. The surface expression of $\operatorname{TACTILE}(\mathbf{A}, \mathbf{B})$ and DNAM-1 (C, D) was analyzed by flow cytometry. The frequency of TACTILE+ T cells remains unaltered upon LAQ treatment $(n=10$ per group, $p>0.05$, unpaired t-test) (B). DNAM- $1+T$ cells are downregulated by LAQ ( $n=10$ per group, ${ }^{*} p<0.05$, unpaired t-test) (D). Data are presented as mean \pm SEM.

Moreover, this set of experiments investigated effects of LAQ on the expression of TIGIT on CD3+ T cells (FIGURE 16A). Other than NK cells, T cells did not downregulate TIGIT in response to LAQ. The frequency of TIGIT+ T cells was comparable in LAQ-treated (mean $2.7 \% \pm 0.2 \%$ ) and vehicle-treated animals (mean $3.0 \% \pm 0.3 \%, p>0.05$ ) (FIGURE 16B). 
A

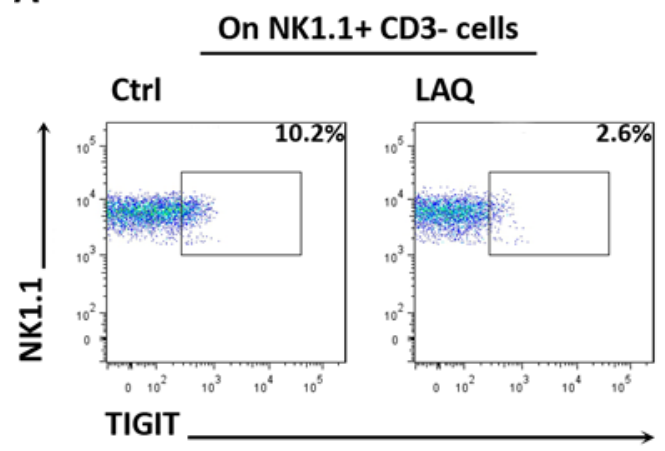

C

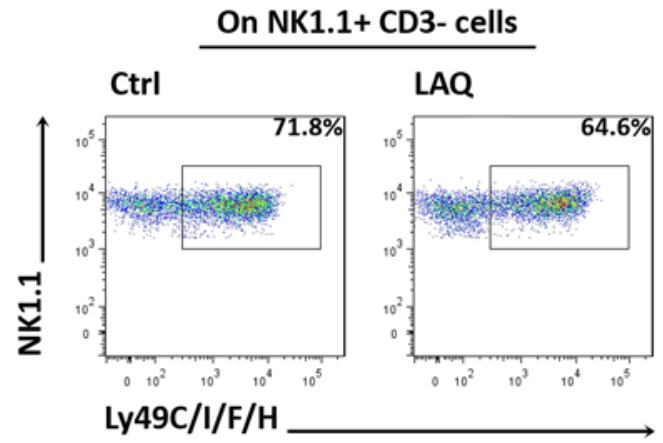

B

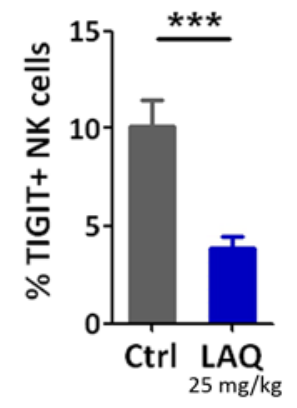

D

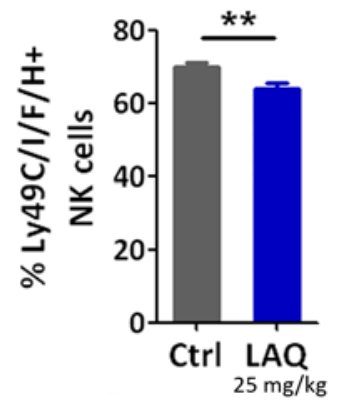

FIGURE 15: LAQ reduces the expression of inhibitory NK cell receptors

The effects of LAQ on inhibitory NK cell receptors were studied ex vivo in the spleens of mice immunized with $M O_{35-55}$ and treated with LAQ (25 mg/kg) (blue) or vehicle (gray) for eleven days in vivo. The surface expression of TIGIT (A, B) and Ly49C/I/F/H (C, D) was analyzed by flow cytometry. LAQ treatment significantly decreases the proportion of TIGIT+ NK cells ( $n=10$ per group, ${ }^{* * *} p<0.001$, unpaired t-test) (B) and $\mathrm{Ly} 49 \mathrm{C} / \mathrm{I} / \mathrm{F} / \mathrm{H}+\mathrm{NK}$ cells ( $\mathrm{n}=10$ per group, ${ }^{* *} \mathrm{p}<0.01$, unpaired $\mathrm{t}$-test) (D). Data are presented as mean \pm SEM.

A

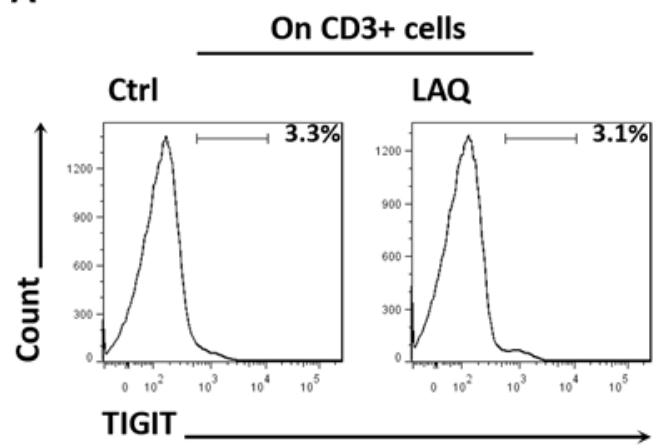

B

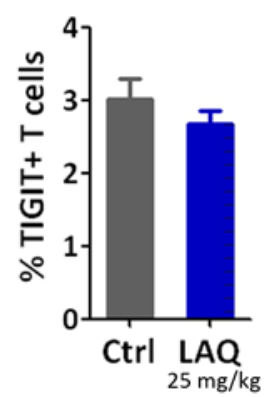

FIGURE 16: LAQ does not regulate TIGIT on T cells

Treatment effects on the expression of TIGIT on T cells were investigated ex vivo in the spleens of mice immunized with $\mathrm{MOG}_{35-55}$ and treated with LAQ $(25 \mathrm{mg} / \mathrm{kg})$ (blue) or vehicle (gray) for eleven days

in vivo. The surface expression of TIGIT (A) was analyzed by flow cytometry. The proportion of TIGIT+ T cells remains constant upon LAQ treatment ( $n=10$ per group, $p>0.05$, unpaired t-test) (B). Data are presented as mean \pm SEM. 
Taken together, LAQ treatment led to the upregulation of activating receptors and the downregulation of inhibitory receptors on NK cells. In detail, LAQ specifically altered the receptor status of NK cells for the recognition of CD155. The surface molecule CD155, which is expressed on endothelial cells, different leukocytes and a variety of tumor cells, regulates NK cell activation by interacting with the activating receptors DNAM-1 and TACTILE and the inhibitory receptor TIGIT (Bottino et al., 2003, Tahara-Hanaoka et al., 2004, Maier et al., 2007, Yu et al., 2009). DNAM-1 and TACTILE showed an increased expression on NK cells upon LAQ treatment, whereas TIGIT was diminished. This LAQ-induced alteration was specific for NK cells, as LAQ showed different effects on these three receptors on T cells.

\subsubsection{In vivo LAQ treatment increased NK cell effector functions ex vivo}

To investigate whether the LAQ-induced changes on NK cell subpopulations and activation state were paralleled by alterations in NK cell function, NK cell effector functions were tested ex vivo. Splenocytes were isolated from $\mathrm{MOG}_{35-55}$-immunized animals pre-treated with either $25 \mathrm{mg} / \mathrm{kg}$ LAQ or vehicle for eleven days in vivo. NK cells were purified by MACS and FACS sorting and subsequently LAQ-induced alterations in NK cell cytotoxicity and IFNY production were assessed in vitro.

\subsubsection{LAQ enhanced the killing efficiency of NK cells towards tumor cells in vitro}

One important function of NK cells is the recognition and the direct killing of tumor cells (Zamai et al., 2007, Vivier et al., 2011). This effector function can be tested in vitro by co-culturing NK cells with NK cell-sensitive tumor cells. The efficiency of NK cell-mediated tumor lysis is one characteristic functional feature depending on the subpopulation of NK cells. It has been described for human NK cells that CD11b+ NK cells are more effective in killing tumor cells in vitro compared to CD27+ NK cells (Fu et al., 2011).

To assess LAQ-mediated changes in the killing efficiency of NK cells towards the B16F10 melanoma cell line in vitro, CD27+ and CD11b+ NK cells (effector cells) isolated from LAQ- or vehicle-treated mice were co-cultures with B16F10 cells (target cells) at different effector/target ratios, ranging from 5:1 to $0.5: 1$. Killing of tumor cells was analyzed using a crystal violet staining. The results are presented as percentage of the B16F10 tumor cells which were lysed by NK cells during the co-culture (percent specific lysis).

The overall comparison of $\mathrm{CD} 27+$ and $\mathrm{CD} 11 \mathrm{~b}+\mathrm{NK}$ cells, irrespective of a prior LAQ treatment, revealed that CD11b+ NK cells displayed a significantly higher capacity to lyse B16F10 
tumor cells in vitro than CD27+ NK cells at all effector/target ratios investigated (FIGURE 17A). TABLE 19 summarizes sample size, mean specific tumor cell lysis \pm SEM and $p$-values for the two NK cell subpopulations at all effector/target ratios.

TABLE 19: CD11b+ NK cells display higher cytotoxicity towards B16F10 cells in vitro than CD27+ NK cells at all effector/target ratios

\begin{tabular}{cccc}
\hline & \multicolumn{2}{c}{ Specific tumor cell lysis [\%] } & \\
\cline { 2 - 3 } Effector/target ratio & CD27+ NK cells & CD11b+ NK cells & \\
\hline $5: 1$ & $\mathrm{n}=13$ & $\mathrm{n}=14$ & $\mathrm{p}$-value \\
\hline $2.5: 1$ & $68.7 \% \pm 2.1 \%$ & $74.2 \% \pm 1.6 \%$ & $\mathrm{p}<0.05$ \\
$1: 1$ & $54.2 \% \pm 2.2 \%$ & $66.8 \% \pm 2.3 \%$ & $\mathrm{p}<0.001$ \\
$0.5: 1$ & $29.3 \% \pm 3.4 \%$ & $51.8 \% \pm 2.8 \%$ & $\mathrm{p}<0.001$ \\
& $15.3 \% \pm 2.5 \%$ & $30.4 \% \pm 2.6 \%$ & $\mathrm{p}<0.001$ \\
\hline
\end{tabular}

Data are presented as mean \pm SEM.

To investigate LAQ-induced alterations in the ability of NK cells to lyse B16F10 melanoma cells, each NK cell subpopulation was analyzed separately. LAQ treatment significantly increased the specific lysis of $C D 27+$ as well as CD11b+ NK cells at the highest effector/target ratio $(5: 1)$. For CD27+ NK cells LAQ rose the mean specific tumor cell lysis from $62.8 \% \pm 2.5 \%$ in controls to $73.7 \%$ $\pm 1.7 \%$ in treated animals $(p<0.05)$ (FIGURE 17B). The mean specific tumor cell lysis by CD11b+NK cells was augmented from $70.7 \% \pm 1.9 \%$ in controls to $78.8 \% \pm 1.4 \%$ in treated animals $(p<0.01)$ (FIGURE 17C). At the lower effector/target ratios LAQ treatment also seemed to enhance tumor cell lysis by both NK cell subsets, but this effect did not reach statistical significance (FIGURE 17B $\& C)$. 
A

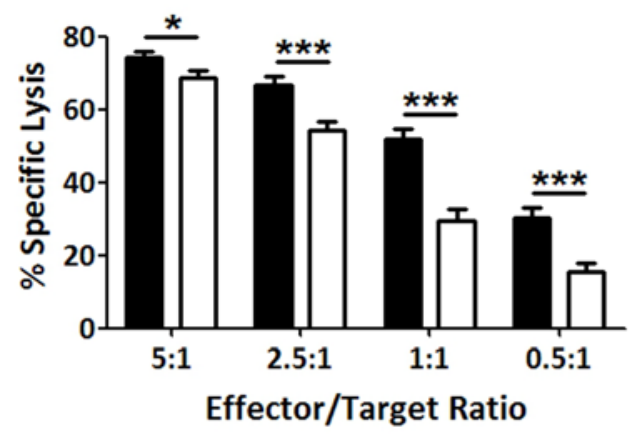

B

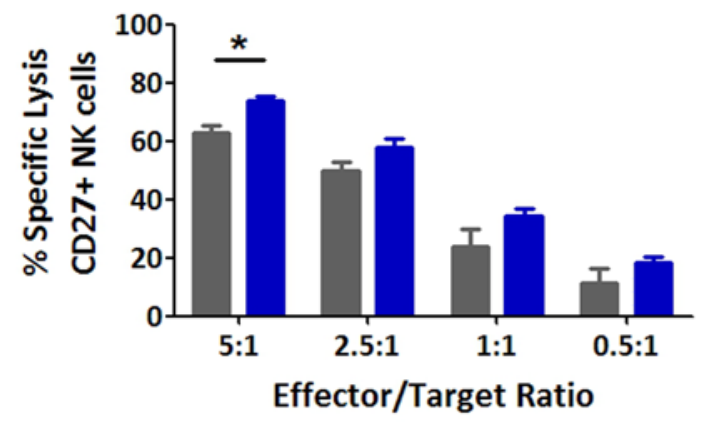

C

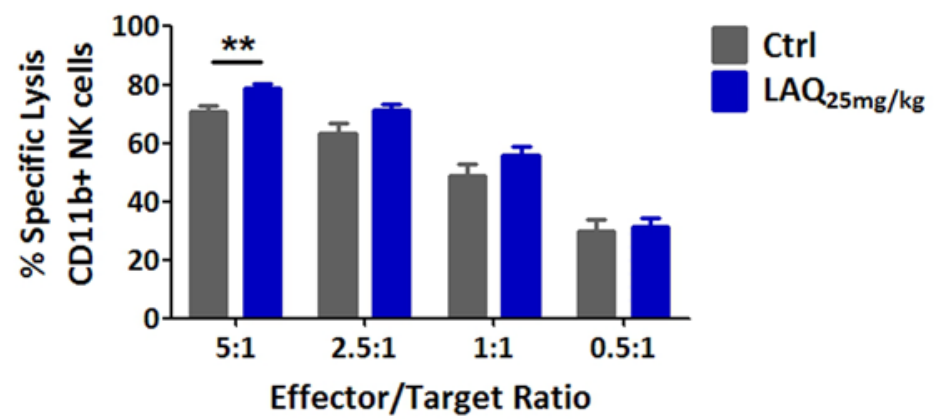

FIGURE 17: LAQ increases the NK cell-mediated killing of tumor cells in vitro

The killing efficiency of NK cells towards the B16F10 melanoma cell line in vitro was investigated using the crystal violet assay. NK cells were purified from the spleen of animals immunized with $\mathrm{MOG}_{35-55}$ and treated with LAQ ( $25 \mathrm{mg} / \mathrm{kg}$ ) (blue) or vehicle (gray) for eleven days in vivo. NK cells (effector) were co-cultured with B16F10 melanoma cells (target) at different effector/target ratios for 48 h. Surviving tumor cells were stained with crystal violet and the specific lysis of tumor cells [\%] was calculated. The overall comparison of CD27+ and CD11b+ NK cells reveals a significantly higher killing efficiency of CD11b+ NK cells at all effector/target ratios investigated (CD27+ NK cells: $n=13, \quad$ CD11b+ NK cells: $n=14$, * $\mathrm{p}<0.05, * * * \mathrm{p}<0.001$, unpaired $\mathrm{t}-$ test) (A). At the highest effector/target ratio LAQ-treated NK cells show a significantly increased killing efficiency compared to control NK cells. This effect is observed for CD27+ NK cells (Ctrl: $n=6$, LAQ: $n=7,{ }^{* *} p<0.01$, unpaired t-test) (B) and CD11b+NK cells (Ctrl: $n=6, L A Q: n=7,{ }^{* *} p<0.01$, Mann Whitney $U$ test) (C). Data are presented as mean \pm SEM.

\subsubsection{LAQ increased IFN $p$-producing NK cells upon stimulation ex vivo}

The second key effector function of NK cells is the production and secretion of different cytokines upon stimulation. One prototypical marker cytokine of NK cells is IFNY (Vivier et al., 2011).

To study effects of LAQ on the production of IFN $\gamma$ in NK cells, NK cells were purified from $\mathrm{MOG}_{35-55}$-immunized animals with and without $\mathrm{LAQ}$ treatment $(25 \mathrm{mg} / \mathrm{kg})$ in vivo. Ex vivo, NK cells 
were stimulated with IL-12 and IL-18. After stimulation IFNY levels were measured in the supernatant by ELISA and the frequency of IFN $\gamma+$ NK cells was assessed by flow cytometry (FIGURE 18A).

The mean frequency of IFN $\gamma+$ NK cells was significantly higher in LAQ-treated animals (mean $74.2 \% \pm 2.2 \%$ ) compared to controls (mean $67.0 \% \pm 1.5 \%, \mathrm{p}<0.05$, unpaired $T$ test) upon stimulation ex vivo (FIGURE 18B).

The quantification of IFNY levels in the supernatants of these stimulated NK cells revealed an increased IFNy secretion in LAQ-treated animals. Supernatants of LAQ-treated samples (mean $43.2 \mathrm{ng} / \mathrm{ml} \pm 2.5 \mathrm{ng} / \mathrm{ml}$ ) contained a 1.7 fold higher IFNY concentration than control samples (mean $25.4 \mathrm{ng} / \mathrm{ml} \pm 1.2 \mathrm{ng} / \mathrm{ml}, \mathrm{p}<0.001$ ) (FIGURE 18C).

A

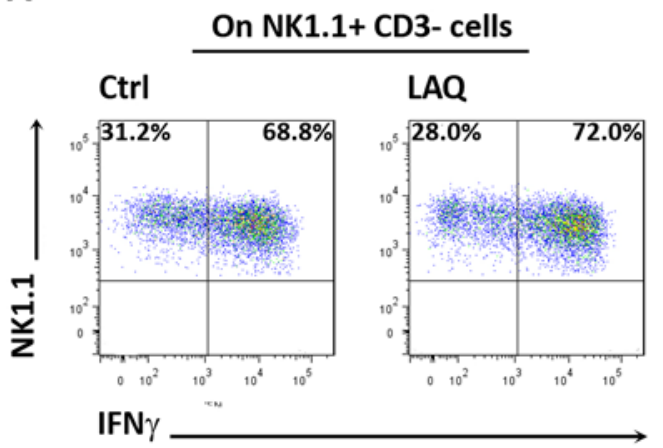

B

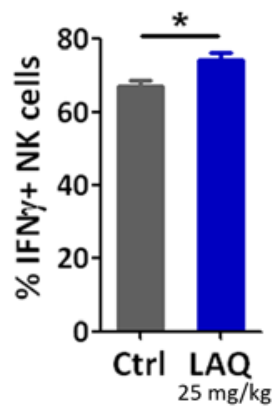

C

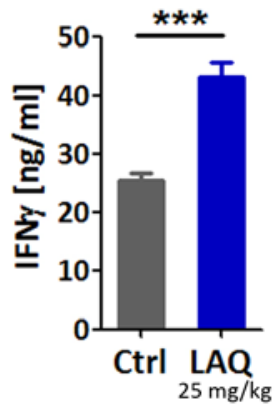

FIGURE 18: LAQ increases IFN $\gamma+$ NK cells

NK cells were purified from the spleens of animals immunized with $M O_{35-55}$ and pre-treated with LAQ (25 $\mathrm{mg} / \mathrm{kg}$ ) (blue) or vehicle (gray) for eleven days in vivo. After in vitro stimulation with IL-12 and IL-18 the proportion of IFN $\gamma+$ NK cells was assessed by intracellular cytokine staining and flow cytometry (A, B) and the IFNY concentration was measured by ELISA in the supernatant (C). Flow cytometric data reveal a LAQdependent rise of IFN ${ }^{+}$NK cells of about $7 \%$ (Ctrl: $n=8$, LAQ: $n=6, * p<0.05$, unpaired t-test) (B). Furthermore, LAQ treatment nearly doubles the IFNy secretion upon stimulation ex vivo (Ctrl: $n=8$, LAQ: $\mathrm{n}=6, * * * \mathrm{p}<0.001$, Mann-Whitney $U$ test) (C). Data are presented as mean \pm SEM. 


\subsection{In vivo LAQ-treated NK cells inhibited antigen-dependent $\mathrm{T}$ cell proliferation in vitro in a contact dependent manner}

NK cells are part of a functional network and interact with $\mathrm{T}$ cells and dendritic cells. Within this network the different cell types influence each other in terms of activation, proliferation and survival. These interactions can be mediated either by direct cell-cell contact or by secretion of pro- and anti-inflammatory cytokines (Vivier et al., 2008, Vivier et al., 2011).

To investigate whether LAQ treatment in vivo could alter the functional properties of NK cells within their network in vitro, NK cells were isolated from mice which were immunized with $M_{30 G}{ }_{35-55}$ and treated with either $25 \mathrm{mg} / \mathrm{kg}$ LAQ or vehicle for eleven days in vivo. The purified NK cells were tested in different co-culture systems to analyze the NK cell effects on T cell proliferation and cell death. Triple co-culture experiments with NK cells, 2D2 T cells and bone marrow-derived dendritic cells were performed to study the effect of LAQ-treated NK cells on antigen-dependent $\mathrm{T}$ cell proliferation and on cell death of $\mathrm{T}$ cells and bone marrow-derived dendritic cells. Further experiments used the triple co-culture setup in a transwell assay to determine, whether NK cell effects on antigen-dependent T cell proliferation were mediated by direct cell contact. Simple co-culture experiments of NK cells with T cells analyzed the effect of LAQ-treated NK cells on antigen-independent T cell proliferation. Simple co-culture setups of NK cells with bone marrow-derived dendritic cells examined the ability of LAQ-treated NK cells to induce cell death in bone marrow-derived dendritic cells.

\subsubsection{In vivo LAQ-treated NK cells reduced antigen-dependent T cell proliferation in a triple co-culture system of NK cells, T cells and bone marrow-derived dendritic cells by direct cell contact}

The effect of in vivo LAQ treatment on NK cell function in vitro was studied in a triple coculture system of NK cells, T cells and bone marrow-derived dendritic cells. In this co-culture, the proliferation of $\mathrm{MOG}_{35-55}$-specific $2 \mathrm{D} 2 \mathrm{~T}$ cells was activated in an antigen-dependent manner using $\mathrm{MOG}_{35-55}$ as antigen and bone marrow-derived dendritic cells as antigen presenting cells. NK cells derived from LAQ- or vehicle-treated animals were added to investigate the effect of these cells on T cell proliferation and cell death. 


\subsubsection{LAQ-treated NK cells reduced $M O_{35-55}$-induced T cell proliferation}

To study $T$ cell proliferation in vitro, 2D2 CD4+ T cells were labeled with the fluorescent dye CFSE prior to antigen-dependent stimulation. CFSE stably crosslinks to proteins within the cytoplasm of T cells. With each cell division, the CFSE fluorescence intensity is then halved in the proliferated cells. By measuring the CFSE profile of 2D2 CD4+ T cells by flow cytometry after $72 \mathrm{~h}$ of stimulation, the maximal number of cell divisions and the proportion of cells in each division state can be analyzed (Parish, 1999).

NK cell- and treatment-related effects on antigen-dependent T cell proliferation were assessed by analyzing the percentage of non-proliferating CD4+ T cells among all CD4+ T cells. Non-proliferating CD4+ $T$ cells were defined as the percentage of $C D 4+T$ cells that displayed maximal CFSE fluorescence intensity (FIGURE 19A).

LAQ-treated NK cells significantly inhibited T cell proliferation compared to the baseline proliferation without NK cells $(p<0.05)$. Moreover, LAQ-treated NK cells inhibited T cell proliferation to a significantly higher extent than control NK cells $(p<0.05)$. Control NK cells also inhibited $\mathrm{T}$ cell proliferation to a certain extent, when compared to the baseline proliferation. However, this effect did not reach statistical significance (FIGURE 19B). TABLE 20 gives a summary of sample size, mean percentage of non-proliferating T cells and SEM.

TABLE 20: NK cells from LAQ-treated animals inhibit antigendependent $\mathrm{T}$ cell proliferation in vitro

\begin{tabular}{ll}
\hline Treatment & $\begin{array}{l}\text { Percentage of non-proliferating } \\
\text { CD4+ T cells }\end{array}$ \\
\hline No NK cells $(n=3)$ & $6.3 \% \pm 1.9 \%$ \\
NK cells Ctrl $(n=11)$ & $10.2 \% \pm 1.1 \%$ \\
NK cells LAQ $(n=12)$ & $19.1 \% \pm 1.8 \%$ \\
\hline
\end{tabular}

Statistical significances:

No NK cells vs. NK cells LAQ (* $p<0.05)$

NK cells Ctrl vs. NK cells LAQ (* $p<0.05)$

Data are presented as mean \pm SEM. 
A

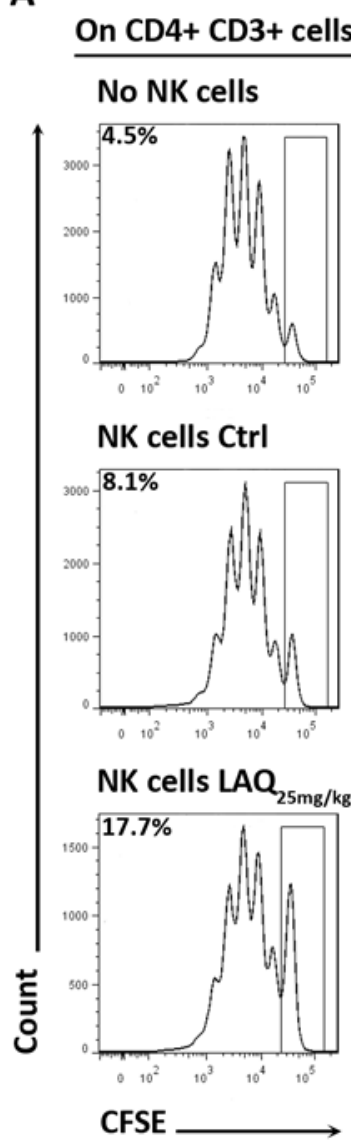

B

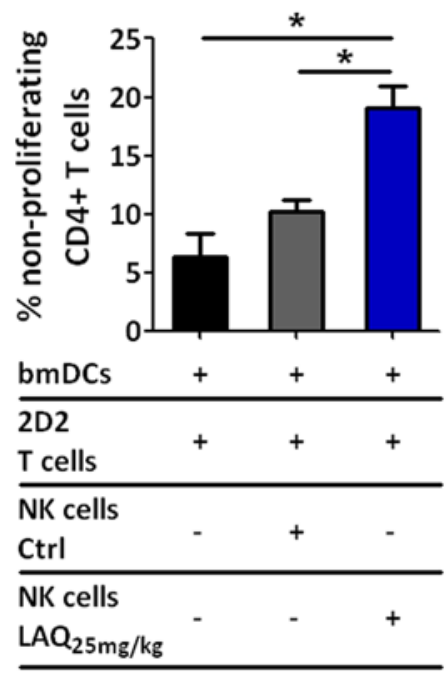

FIGURE 19: LAQ-treated NK cells inhibit T cell proliferation

NK cells were purified from the spleens of animals immunized with $\mathrm{MOG}_{35-55}$ and pre-treated with LAQ (25 mg/kg) (blue) or vehicle (gray) for eleven days in vivo. In vitro, these NK cells were co-cultured with bone marrow-derived dendritic cells and CFSE-labeled 2D2 T cells. T cell proliferation was induced by adding $20 \mu \mathrm{g} / \mathrm{ml}$ $M_{35-55}$ to the culture. The proliferation was analyzed by flow cytometry in the absence of NK cells and in the presence of either LAQ-treated or control NK cells (A). LAQ-treated NK cells $(n=12)$ significantly increase the proportion of non-proliferating CD4+ $\mathrm{T}$ cells compared to samples without NK cells $(n=3, * p<0.05$, KruskalWallis test with Dunn's post test) and control NK cells $(n=11, * p<0.05$, KruskalWallis test with Dunn's post test) (B). Data are presented as mean \pm SEM.

\subsubsection{The NK cell-mediated inhibition of $\mathrm{MOG}_{35-55}$-induced $\mathrm{T}$ cell proliferation was dependent on direct cell contact}

NK cell-dependent inhibition of $\mathrm{T}$ cell proliferation can be mediated by different mechanisms, either involving direct cell-cell contact or cytokine secretion.

To investigate whether the NK cell-mediated inhibition of T cell proliferation shown in 3.4.1.1 was dependent on direct cell contact, the triple co-culture experiment was repeated in transwell plates. In these plates, each well consisted of two different compartments separated by a membrane, which allowed diffusion of soluble factors like cytokines, but prevented cell migration. NK cells, purified from mice immunized with $\mathrm{MOG}_{35-55}$, were either added to the same compartment as $\mathrm{T}$ cells and dendritic cells, allowing interactions by direct cell contact and cytokine secretion or they were added to the transwell compartment, inhibiting direct cell contact, but enabling the communication via soluble factors. 
Contact-dependent versus contact-independent effects of NK cells on antigen-dependent $\mathrm{T}$ cell proliferation were again assessed by analyzing the percentage of non-proliferating CD4+ T cells among all CD4+ T cells (FIGURE 20A).

NK cell-mediated inhibition of T cell proliferation was dependent on direct cell contact. NK cells did not inhibit $T$ cell proliferation when separated from $T$ cells and dendritic cells by the transwell membrane. Only co-culture with NK cells in the same compartment together with activated $T$ cells and dendritic cells led to a significant increase in the frequency of nonproliferating CD4+ $T$ cells compared to the baseline value where no NK cells were added to the culture $(p<0.05)$ and compared to samples where NK cells were separated by the transwell membrane $(p<0.001)$ (FIGURE 20B). TABLE 21 summarizes sample size, mean percentage of nonproliferating CD4+ T cells and SEM.

A
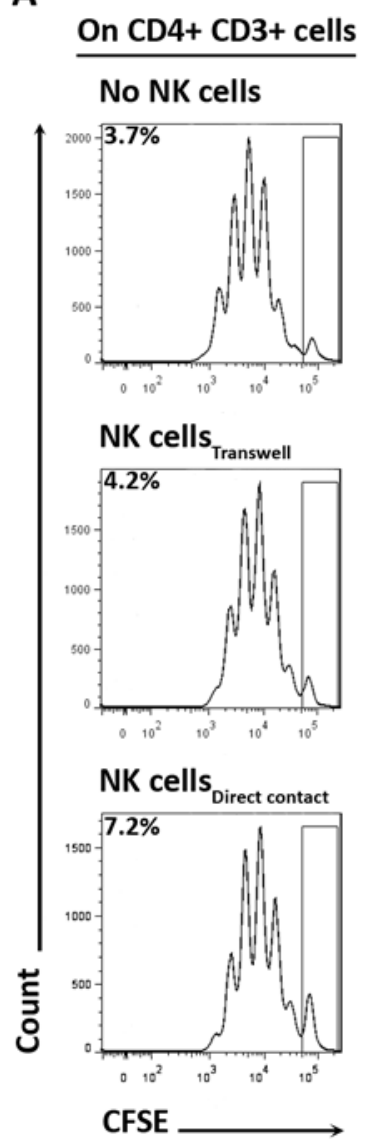

B

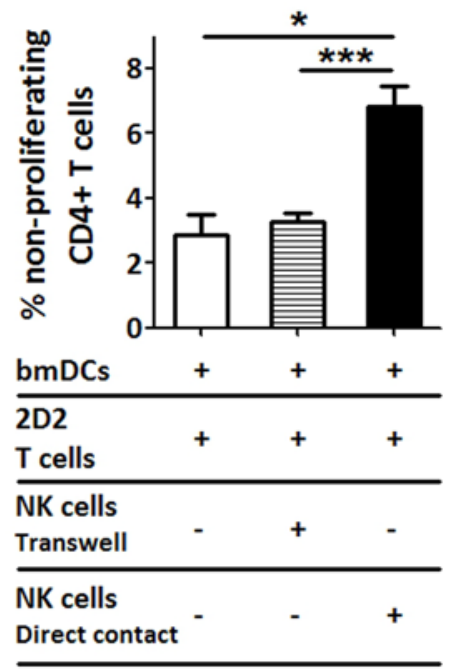

FIGURE 20: NK cell-mediated inhibition of T cell proliferation is contact-dependent

NK cells were purified from the spleens of animals immunized with $\mathrm{MOG}_{35-55}$. In vitro, these NK cells were co-cultured with bone marrow-derived dendritic cells and CFSE-labeled 2D2 T cells either in direct contact or separated by a transwell membrane. T cell proliferation was induced by adding $20 \mu \mathrm{g} / \mathrm{ml} \mathrm{MOG}_{35-55}$ to the culture. Proli-feration was analyzed in samples without NK cells, with NK cells in direct contact and with NK cells separated from $T$ cells and dendritic cells by a membrane using flow cytometry (A). NK cells which are separated from $T$ cells and dendritic cells by the transwell membrane $(n=24)$ do not inhibit $T$ cell proliferation, whereas NK cells in direct contact with T

cells and dendritic cells $(n=24)$ significantly increase the proportion of non-proliferating CD4+ T cells compared to samples without NK cells $(n=4, * p<0.05$, one way ANOVA with Bonferroni post test) and to NK cells in the transwell ( ${ }^{* * *} \mathrm{p}<0.001$, one way ANOVA with Bonferroni post test) (B). Data are presented as mean \pm SEM. 
TABLE 21: NK cells inhibit MOG $_{35-55}$-induced $T$ cell proliferation in a contact-dependent way

\begin{tabular}{ll}
\hline Culture condition & $\begin{array}{l}\text { Percentage of non-proliferating } \\
\text { CD4+ T cells }\end{array}$ \\
\hline No NK cells $(n=4)$ & $2.9 \% \pm 0.6 \%$ \\
NK cells transwell $(n=24)$ & $3.3 \% \pm 0.3 \%$ \\
NK cells direct contact $(n=24)$ & $6.8 \% \pm 0.7 \%$ \\
\hline
\end{tabular}

Statistical significances:

No NK cells vs. NK cells direct contact $(* p<0.05)$

NK cells transwell vs. NK cells direct contact $(* * * p<0.001)$

Data are presented as mean \pm SEM.

\subsubsection{NK cells induced cell death in T cells and bone marrow-derived dendritic cells irrespective of in vivo LAQ treatment}

One mechanism by which NK cells could inhibit T cell proliferation in a contact-dependent manner is the induction of cell death in T cells and/or dendritic cells.

To investigate whether NK cells induced cell death of activated T cells and dendritic cells in the triple co-culture and if this effect was LAQ-dependent, cells were stained with Annexin $V$ and 7-aminoactinomycin D (7-AAD) after $18 \mathrm{~h}$ of co-culture.

Annexin $\mathrm{V}$, which binds to the phospholipid component phosphatidylserine, is a marker for early and late apoptotic/dead cells. 7-AAD, an intercalating substance that binds to GC regions of the DNA, strongly labels late apoptotic/dead cells. Both molecules are generally excluded from viable cells by an intact plasma membrane.

In viable cells, phosphatidylserine is located at the cytosolic side of the lipid bilayer of the cell membrane. As an early event during apoptosis phosphatidylserine is moved from the inner leaflet of the plasma membrane to the outer leaflet, but the plasma membrane remains mostly intact. At this early stage of apoptosis, Annexin $\mathrm{V}$ is able to bind to phosphatidylserine at the cell surface, but only low amounts of 7-AAD enter the cell. During late stages of apoptosis or during necrotic cell death, the plasma membrane becomes permeable. Then Annexin $V$ and 7-AAD can enter the cell efficiently and bind to their targets (Vermes et al., 1995, Philpott et al., 1996, Zhang et al., 1997).

Annexin $\mathrm{V}$ and 7-AAD staining of CD3+ T cells (FIGURE 21A) and MHCII+ CD11 $\mathrm{c}^{\text {high }}$ dendritic cells (FIGURE 22A) was analyzed by flow cytometry. Annexin V- 7-AAD- cells were classified as viable and Annexin V+ 7-AAD- cells as early apoptotic. Cells that were either Annexin V+ 7AAD+ or 
Annexin V- 7-AAD+ were both classified as late apoptotic/dead. Data were analyzed as percentage of apoptotic and percentage of late apoptotic/dead cells among all CD3+ T cells and MHCll+ CD11c ${ }^{\text {high }}$ dendritic cells.

NK cells did not increase the proportion of apoptotic T cells (FIGURE 21B) and dendritic cells (FIGURE 22B) irrespective of any treatment with LAQ in vivo at the time point analyzed.

In contrast, the addition of NK cells to the culture considerably increased the frequency of late apoptotic/dead T cells from about $10 \%$ without NK cells to nearly $20 \%$. Similar induction rates of cell death were observed in co-cultures with LAQ-treated NK cells $(p<0.001)$ and control NK cells $(p<0.001)$ (FIGURE 21C).

In dendritic cells, the frequency of late apoptotic/dead cells was also markedly increased upon co-culture with NK cells from $16 \%$ to about $27 \%$. LAQ-treated NK cells $(p<0.001)$ and control NK cells $(p<0.001)$ triggered cell death to a comparable degree (FIGURE 22C). TABLE 22 summarizes sample sizes, mean percentages of apoptotic $T$ cells, late apoptotic/dead T cells, apoptotic dendritic cells and late apoptotic/dead dendritic cells with the corresponding SEM.

TABLE 22: NK cells trigger cell death in T cells and bone marrow-derived dendritic cells independent of LAQ treatment in vivo

\begin{tabular}{lccc}
\hline & \multicolumn{3}{c}{ Treatment } \\
\cline { 2 - 4 } & No NK cells & NK cells Ctrl & NK cells LAQ \\
& $(n=4)$ & $(n=8)$ & $(n=8)$ \\
\hline Percentage of apoptotic T cells & $5.7 \% \pm 0.5 \%$ & $6.4 \% \pm 0.3 \%$ & $6.3 \% \pm 0.2 \%$ \\
Percentage of late apoptotic/dead T cells & $9.9 \% \pm 0.6 \%$ & $19.3 \% \pm 0.6 \%$ & $19.4 \% \pm 0.3 \%$ \\
Percentage of apoptotic dendritic cells & $35.1 \% \pm 2.6 \%$ & $32.7 \% \pm 1.2 \%$ & $30.1 \% \pm 0.8 \%$ \\
Percentage of late apoptotic/dead dendritic cells & $16.2 \% \pm 0.5 \%$ & $28.1 \% \pm 1.0 \%$ & $26.8 \% \pm 0.5 \%$ \\
\hline
\end{tabular}

\section{Statistical significances:}

Late apoptotic/dead T cells:

No NK cells vs. NK cells Ctrl (*** $p<0.001)$, No NK cells vs. NK cells LAQ $(* * * p<0.001)$

Late apoptotic/dead dendritic cells:

No NK cells vs. NK cells Ctrl ( $\left.{ }^{* *} p<0.001\right)$, No NK cells vs. NK cells LAQ $(* * * p<0.001)$

Data are presented as mean \pm SEM. 
A

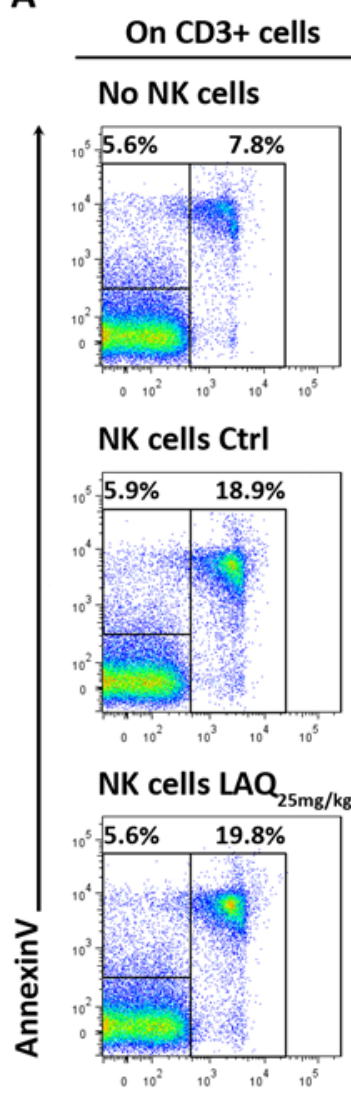

B
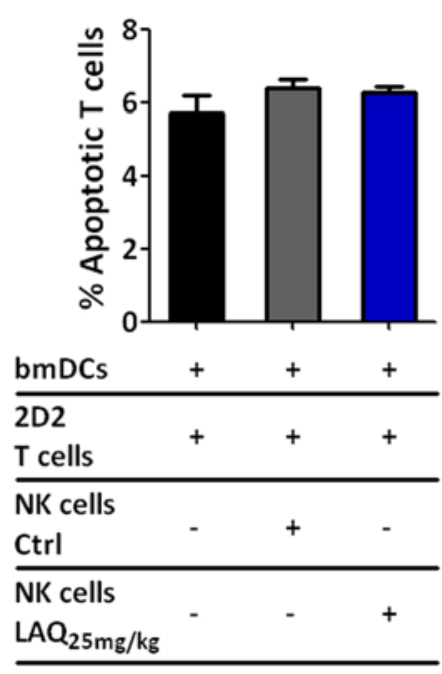

C

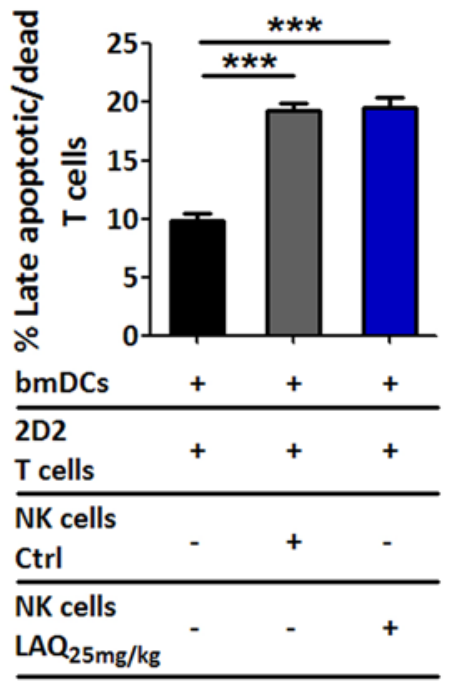

7-AAD

\section{FIGURE 21: NK cells induce cell death in T cells in the triple co-culture system}

NK cells were purified from the spleens of animals immunized with $\mathrm{MOG}_{35-55}$ and pre-treated with LAQ $(25 \mathrm{mg} / \mathrm{kg}$ ) (blue) or vehicle (gray) for eleven days in vivo. In vitro, these NK cells were co-cultured with bone marrow-derived dendritic cells and 2D2 T cell. T cell proliferation was activated by adding $\mathrm{MOG}_{35-55}$ to the culture. Flow cytometry for Annexin V and 7-AAD staining was used to analyze the induction of cell death in the absence of NK cells and in the presence of either LAQ-treated or control NK cells (A). The addition of NK cells, LAQ-treated $(n=8)$ or vehicle-treated $(n=8)$, does not increase the proportion of early apoptotic T cells ( $p<0.05$, one way ANOVA with Bonferroni post test) (B). In contrast, the proportion of late apoptotic/dead T cells is significantly augmented upon co-culture with NK cells. LAQ-treated $(* * * p<0.001$, one way ANOVA with Bonferroni post test) and control NK cells (*** $p<0.001$, one way ANOVA with Bonferroni post test) lead to similar cell death rates of T cells (C). Data are presented as mean \pm SEM. 
A

On MHCII+ CD11 $\mathrm{c}^{\text {high }}$ cells

No NK cells

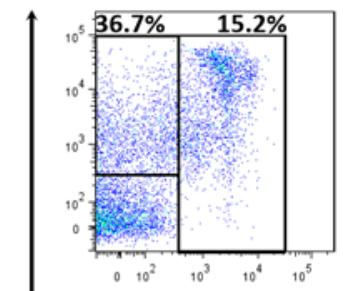

NK cells Ctrl

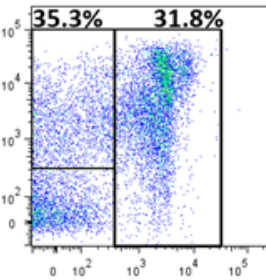

NK cells $\mathrm{LAQ}_{25 \mathrm{mg} / \mathrm{kg}}$

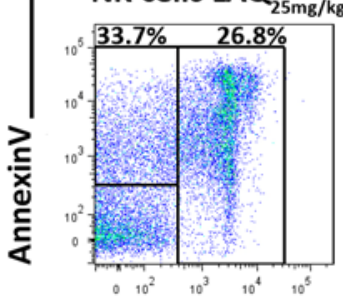

B
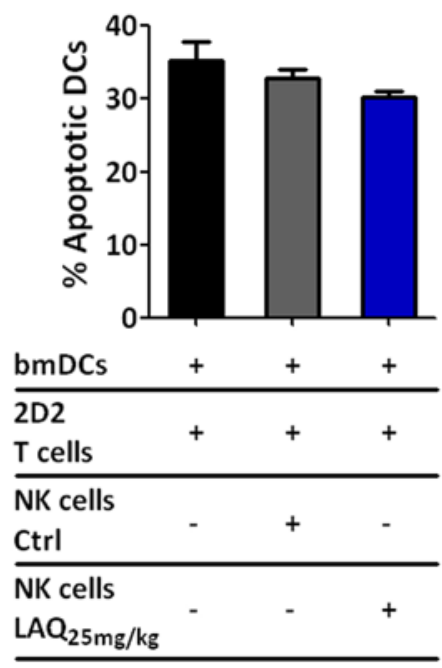

C

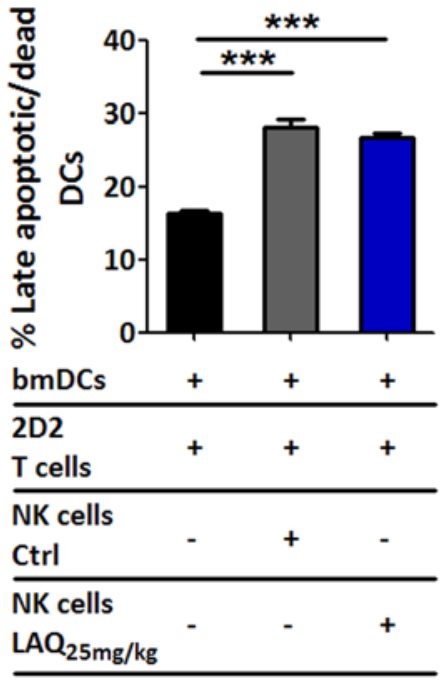

7-AAD

FIGURE 22: NK cells induce cell death in dendritic cells in the triple co-culture system

NK cells were purified from the spleens of animals immunized with $\mathrm{MOG}_{35-55}$ and pre-treated with LAQ $(25 \mathrm{mg} / \mathrm{kg}$ ) (blue) or vehicle (gray) for eleven days in vivo. In vitro, these NK cells were co-cultured with bone marrow-derived dendritic cells and 2D2 T cell. T cell proliferation was activated by adding $\mathrm{MOG}_{35-55}$ to the culture. Flow cytometry for Annexin V and 7-AAD staining was used to analyze the induction of cell death in the absence of NK cells and in the presence of either LAQ-treated or control NK cells (A). Dendritic cells in the early phase of apoptosis are not increased after adding LAQ-treated $(n=8)$ or vehicle-treated $(n=8)$ NK cells ( $p<0.05$, one way ANOVA with Bonferroni post test) (B). As observed for T cells (compare FIGURE 21), the population of late apoptotic/dead dendritic cells is profoundly enlarged upon co-culture with NK cells. LAQ-treated (*** $p<0.001$, one way ANOVA with Bonferroni post test) and control NK cells $(* * * \mathrm{p}<0.001$, one way ANOVA with Bonferroni post test) lead to similar cell death rates in dendritic cells (C). Data are presented as mean \pm SEM.

\subsubsection{NK cells did not affect antigen-independent $\mathrm{T}$ cell proliferation in a co-culture system with T cells}

LAQ-treated NK cells caused a significant inhibition of T cell proliferation in the triple coculture system with 2D2 T cells and bone marrow-derived dendritic cells (compare 3.4.1.1 and 
FIGURE 19). To investigate whether this effect was dependent on the presence of bone marrowderived dendritic cells, the next set of experiments induced T cell proliferation in an antigenindependent manner in a culture without dendritic cells. The proliferation of CFSE-labeled CD4+ T cells, isolated from naïve C57BI/6J mice, was stimulated via agonistic anti-CD3 and anti-CD28 antibodies, whereby either no NK cells, LAQ-treated NK cells or control NK cells were added to the culture. The CFSE profile was measured after $72 \mathrm{~h}$ using flow cytometry. Results are shown as the percentage of $\mathrm{CD} 4+\mathrm{T}$ cells with low proliferation rate versus the percentage of $\mathrm{CD} 4+\mathrm{T}$ cells with high proliferation rate. Low proliferation rate was defined as no, one, two and three cell divisions and high proliferation rate as four cell divisions or more (FIGURE 23A).

Neither control NK cells nor LAQ-treated NK cells reduced CD4+ T cell proliferation after antigen-independent stimulation. The frequencies of $C D 4+T$ cells with low proliferation rate were similar in samples without NK cells (mean $64.2 \% \pm 4.4 \%$ ), samples with control NK cells (mean $57.6 \% \pm 5.2, p>0.05$ ) and samples with LAQ-treated NK cells (mean $54.8 \% \pm 5.5 \%, p>0.05$ ) (FIGURE 23B). The comparison of the frequencies of CD4+ $T$ cells with high proliferation rate did not reveal any significant differences between samples without NK cells (mean $35.8 \% \pm 4.4 \%$ ), with control NK cells (mean $41.7 \% \pm 5.1, p>0.05$ ) and with LAQ-treated NK cells (mean $44.5 \% \pm 5.3 \%, p>0.05$ ) either (FIGURE 23C).

\subsubsection{NK cells did not kill bone marrow-derived dendritic cells in a co-culture system}

NK cells induced cell death in T cells and bone marrow-derived dendritic cells in the triple co-culture system (compare 3.4.1.3 and FIGURE 21 and FIGURE 22). To assess whether LAQtreated NK cells would directly kill bone marrow-derived dendritic cells in the absence of activated T cells, bone marrow-derived dendritic cells (target) with or without preceding LPS preactivation were co-cultured with LAQ-treated or control NK cells (effector) at an effector/target ratio of 1:1. The killing rates of dendritic cells were then analyzed using a crystal violet staining and data were presented as percentage of viable dendritic cells.

Data analysis revealed no significant killing of bone marrow-derived dendritic cells by NK cells, irrespective of prior LPS and LAQ treatment. Dendritic cells without LPS treatment showed a viability of $100 \% \pm 2.7 \%$ when co-cultured with control NK cells and $95.5 \% \pm 2.3 \%$ with LAQtreated NK cells ( $p>0.05$ ) (FIGURE 24A). Similar survival rates of LPS-activated dendritic cells were observed in co-culture with control NK cells $(100 \% \pm 2.7 \%)$ compared to LAQ-treated NK cells $(100 \% \pm 6.8 \% ; p>0.05)$ (FIGURE 24B). 
A

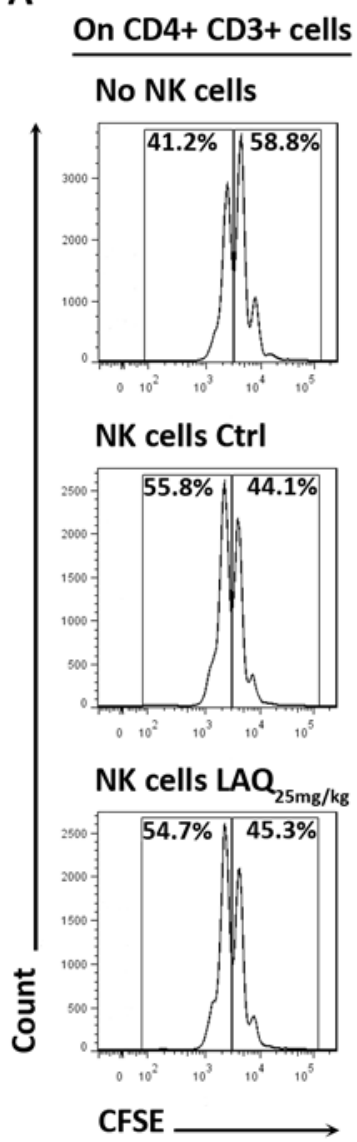

B

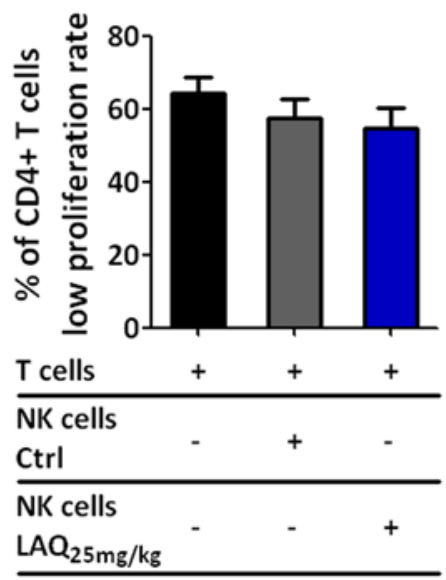

C

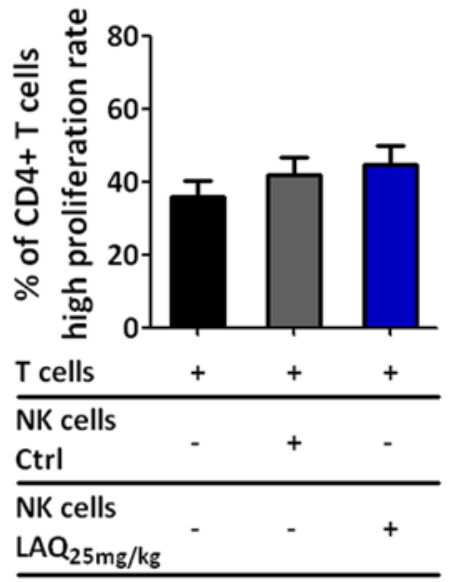

FIGURE 23: NK cells do not interfere with T cell proliferation, when co-cultured with T cells alone

NK cells were purified from the spleens of animals immunized with $M O_{35-55}$ and pre-treated with LAQ $(25 \mathrm{mg} / \mathrm{kg}$ ) (blue) or vehicle (gray) for eleven days in vivo. In vitro, these NK cells were co-cultured with CFSE-labeled CD4+ T cells. T cell proliferation was induced by agonistic anti-CD3 and anti-CD28 antibodies. Proliferation was analyzed by flow cytometry in the absence of NK cells and in the presence of either LAQtreated or control NK cells (A). Addition of NK cells to the culture does not significantly alter T cell proliferation. The proportion of CD4+ T cells with a low proliferation rate is similar in samples without NK cells ( $n=3)$, with LAQ-treated NK cells $(n=7)$ and with control NK cells ( $n=8, p>0.05$, Kruskal-Wallis test with Dunn's post test) (B). Similarly, neither control NK cells $(n=8)$ nor LAQ-treated NK cells influence the frequency of $C D 4+T$ cells with a high proliferation rate ( $p>0.05$, Kruskal-Wallis test with Dunn's post test) (C). Data are presented as mean \pm SEM. 
A

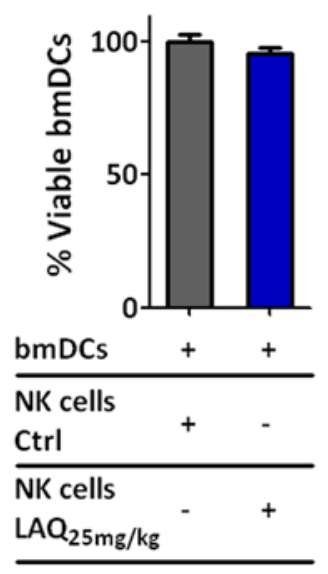

B

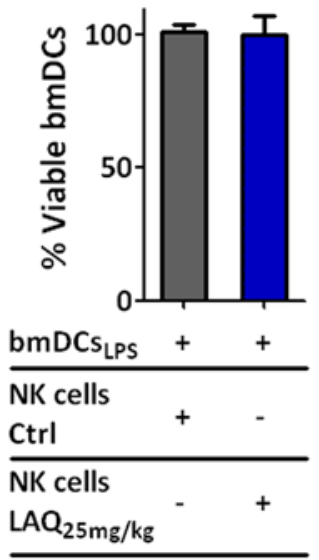

FIGURE 24: NK cells do not kill dendritic cells when co-cultured with dendritic cells alone NK cells were purified from the spleens of animals immunized with $\mathrm{MOG}_{35-55}$ and pretreated with LAQ (25 mg/kg) (blue) or vehicle (gray) for eleven days in vivo. In vitro, these NK cells were co-cultured with bone marrow-derived dendritic cells. Viable dendritic cells were stained with crystal violet. In the absence of activated T cells, NK cells do not induce cell death in bone marrow-derived dendritic cells. Neither LAQ-treated $(n=3)$ nor control NK cells $(n=8)$ kill dendritic cells in this experimental setting. This is shown for both co-culture experiments of NK cells with naïve (A) and LPS-activated dendritic cells (B) ( $p>0.05$, MannWhitney $U$ test).

In summary, NK cells, treated with $25 \mathrm{mg} / \mathrm{kg}$ LAQ for eleven days in vivo, markedly reduced antigen-dependent T cell proliferation (see 3.4.1.1 and FIGURE 19) in a contact-mediated fashion (see 3.4.1.2 and FIGURE 20), when cultured with CD4+ T cells and bone marrow-derived dendritic cells in a triple co-culture system. In vivo LAQ treatment did not increase NK cellmediated killing of T cells and bone marrow-derived dendritic cells in this triple co-culture setting (see 3.4.1.3 and FIGURES 21 and 22). In contrast to the observed inhibitory effect of LAQ-treated NK cells on antigen-dependent T cell proliferation in the triple co-culture, NK cells did not interfere with antigen-independent T cell proliferation in a co-culture with CD4+ T cells alone (see 3.4.2 and FIGURE 23). Furthermore, NK cells - irrespective of prior LAQ treatment - did not induce cell death in bone marrow-derived dendritic cells, when co-cultured with dendritic cells alone in the absence of activated T cells (see 3.4.3 and FIGURE 24). 


\subsection{LAQ-mediated effects on NK cells were independent of dendritic cells and vice versa}

LAQ-mediated effects were demonstrated for different innate immune cells in vivo, namely dendritic cells (compare 3.2) and NK cells (compare 3.3). These two cell types interact in vivo and can influence each other by modulating activation, proliferation and survival.

The following set of experiments investigated whether NK cells and dendritic cells were independent targets of LAQ treatment in vivo or if one effect was secondary to the other. First, time course experiments evaluated the kinetics of LAQ-induced changes in NK cells, dendritic cells and T cells. Moreover, effects of LAQ on dendritic cells were analyzed in NK cell-competent and NK cell-depleted animals, to investigate whether NK cell were essential or dispensable for LAQ action on dendritic cells. Finally, in vitro treatment experiments of naïve NK cells in the absence of any other cell type evaluated a direct LAQ effect on NK cell activation.

\subsubsection{NK cell and dendritic cell responses to LAQ treatment preceded changes in the T cell compartment}

To investigate the kinetics of LAQ-induced changes in NK cells, dendritic cells and T cells, time course experiments were performed.

For this purpose, splenocytes were isolated from $\mathrm{MOG}_{35-55}$-immunized mice, which received $25 \mathrm{mg} / \mathrm{kg}$ LAQ or vehicle for one, two, three or four days. Treatment was started directly before the immunization and was then applied daily. Cells were analyzed using flow cytometry.

Treatment of immunized C57Bl/6J mice with $25 \mathrm{mg} / \mathrm{kg}$ LAQ for eleven days in vivo considerably increased the frequencies of CD69+, TACTILE+ and NKG2D+ NK cells, as described previously in this work (compare 3.3.2.2.1 and 3.3.2.2.2 and FIGURES 12 and 13). Therefore, early LAQ-dependent modulation of NK cells was assessed by analyzing the frequencies of CD69+ (FIGURE 25A), TACTILE+ (FIGURE 25C) and NKG2D+ (FIGURE 25E) NK cells among all NK1.1+ CD3cells.

Data analysis revealed very early effects of LAQ treatment on NK cells. The mean frequency of TACTILE+ NK cells was already increased after one day of LAQ treatment compared to controls $(p<0.01)$. A significant difference between LAQ treatment and control was further observed after two days $(p<0.01)$ and a similar trend after three days of treatment $(p=0.096)$ (FIGURE 25D). CD69+ as well as NKG2D+ NK cells showed a higher frequency in the LAQ-treated group compared to controls after two days of treatment. The proportion of CD69+ NK cells was markedly increased in the LAQ group after two and three days of treatment $(p<0.001)$ (FIGURE 25B). The frequencies of NKG2D+ NK cells were higher in LAQ-treated animals than those 
observed in control animals after two days $(p<0.01)$ and after three days of treatment $(p<0.05)$ (FIGURE 25F). TABLE 23 provides sample sizes, mean percentages of CD69+, TACTILE+ and NKG2D+ NK cells with corresponding SEM and $p$-values.

TABLE 23: LAQ leads to upregulation of the activation-associated molecules CD69, TACTILE and NKG2D on NK cells after one to two days of treatment

\begin{tabular}{|c|c|c|c|}
\hline & \multicolumn{2}{|c|}{ Percentage of CD69+ NK cells } & \multirow[b]{3}{*}{ p-value } \\
\hline & Control & LAQ & \\
\hline Days of treatment & $(n=6)$ & $(n=6)$ & \\
\hline 1 & $10.6 \% \pm 1.6 \%$ & $12.8 \% \pm 2.0 \%$ & $>0.05$ \\
\hline 2 & $9.6 \% \pm 0.8 \%$ & $17.8 \% \pm 1.0 \%$ & $<0.001$ \\
\hline \multirow[t]{3}{*}{3} & $10.6 \% \pm 0.9 \%$ & $28.5 \% \pm 2.3 \%$ & $<0.001$ \\
\hline & \multicolumn{2}{|c|}{ Percentage of TACTILE+ NK cells } & \\
\hline & Control & LAQ & \\
\hline Days of treatment & $(n=3)$ & $(n=3)$ & p-value \\
\hline 1 & $76.8 \% \pm 1.3 \%$ & $85.6 \% \pm 0.4 \%$ & $<0.01$ \\
\hline 2 & $80.5 \% \pm 0.9 \%$ & $88.6 \% \pm 0.7 \%$ & $<0.01$ \\
\hline \multirow[t]{3}{*}{3} & $79.5 \% \pm 1.7 \%$ & $86.9 \% \pm 2.9 \%$ & 0.096 \\
\hline & \multicolumn{2}{|c|}{ Percentage of NKG2D+ NK cells } & \\
\hline & Control & LAQ & \\
\hline Days of treatment & $(n=3)$ & $(n=3)$ & p-value \\
\hline 1 & $93.6 \% \pm 0.3 \%$ & $95.3 \% \pm 1.3 \%$ & $>0.05$ \\
\hline 2 & $87.7 \% \pm 1.2 \%$ & $94.4 \% \pm 0.6 \%$ & $<0.01$ \\
\hline 3 & $91.7 \% \pm 0.3 \%$ & $95.0 \% \pm 1.0 \%$ & $<0.05$ \\
\hline
\end{tabular}

Data are presented as mean \pm SEM. 
A

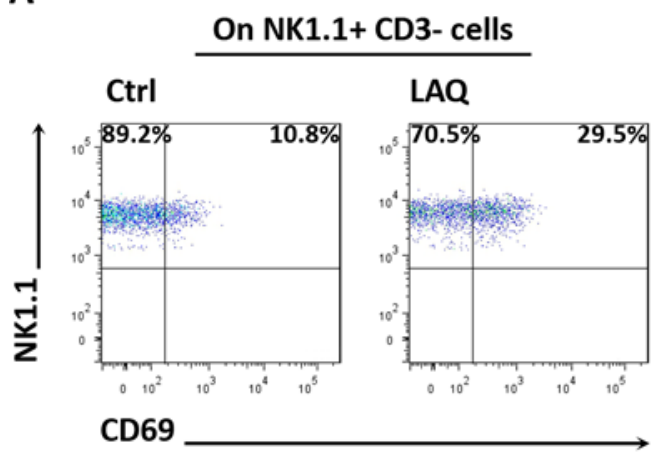

C

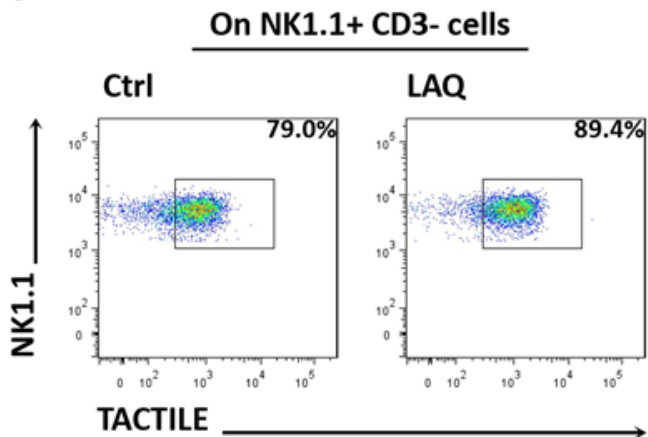

E

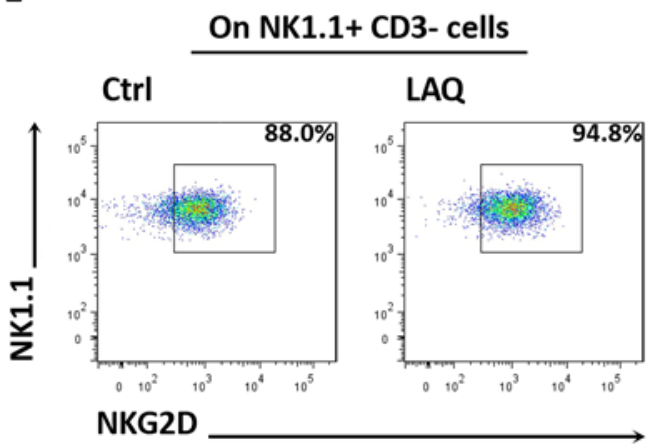

B

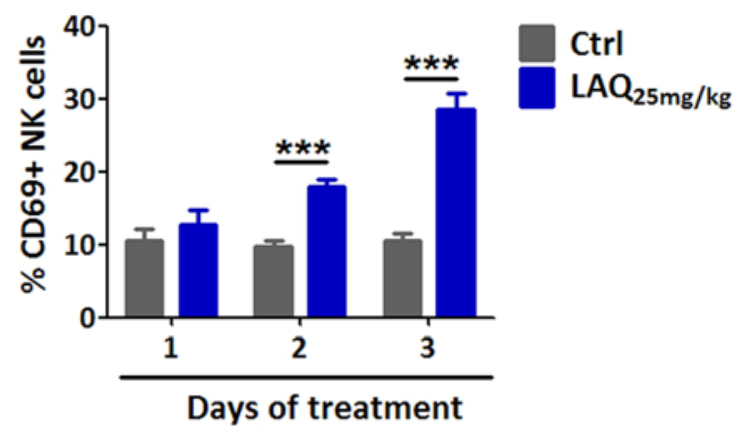

D

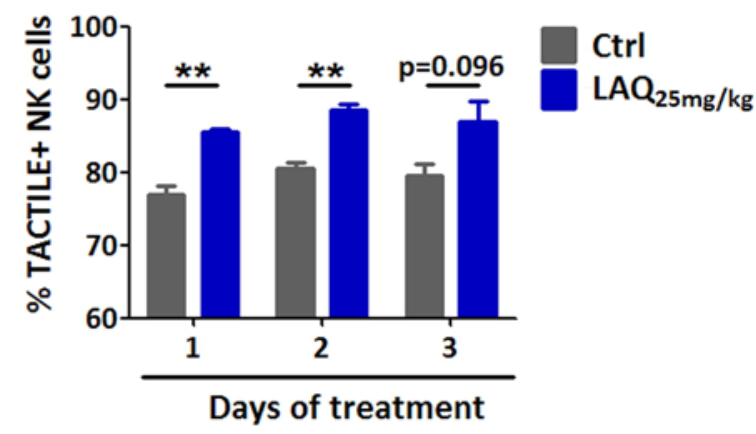

$\mathbf{F}$

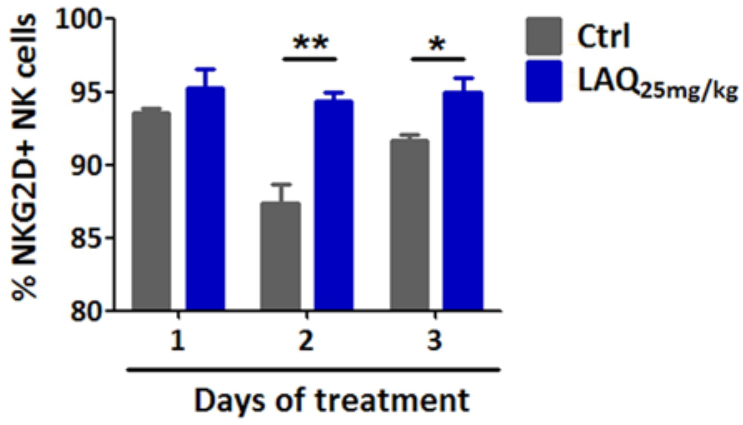

FIGURE 25: LAQ alters surface marker expression on NK cells after one to two days of treatment

Time course experiments were performed to study the kinetics of LAQ-mediated changes in NK cells. Splenocytes were isolated from animals immunized with $M_{35-55}$ and pre-treated with LAQ $(25 \mathrm{mg} / \mathrm{kg})$ (blue) or vehicle (gray) for one, two or three days in vivo. The proportions of CD69+ (A, B), TACTILE+ (C, D) and NKG2D+ cells (E, F) among all NK1.1+ NK cells were analyzed using flow cytometry. LAQ treatment induces an up-regulation of CD69+ NK cells ( $n=6$ per group, ${ }^{* * *} p<0.001$, unpaired t-test) (B) and NKG2D+ NK cells ( $n=3$ per group, ${ }^{* *} p<0.01$, unpaired t-test) $(\mathbf{F}$ ) after two days of treatment. The proportion of TACTILE+ NK cells is already significantly increased after one single dose of LAQ ( $n=3$ per group, ${ }^{* *} p<0.01$, unpaired t-test) (D). Data are presented as mean \pm SEM. 
In this work, eleven days of LAQ treatment markedly reduced the frequency of total splenic dendritic cells in immunized animals (compare 3.2.1 and FIGURE 8). Furthermore, a published study reported early effects of LAQ on the expression of the co-stimulatory molecule CD86 on dendritic cells (Jolivel et al., 2013). Thus, the kinetics of LAQ-induced changes in dendritic cells were evaluated by investigating the total frequency of dendritic cells in the spleen (FIGURE 26A) and the proportions of MHCII+ CD11 $\mathrm{c}^{\text {high }}$ cells expressing the co-stimulatory molecules CD80 (FIGURE 26C) or CD86 (FIGURE 26E).

As for NK cells, LAQ-mediated effects on dendritic cells occurred within the first days of treatment. LAQ led to a significant decrease in the mean frequency of dendritic cells in the spleen after three days of treatment $(p<0.01$ ) (FIGURE 26B). The percentage of CD80+ dendritic cells was considerably higher in LAQ-treated animals compared to controls after two days $(p<0.05)$ and three days of therapy $(p<0.01)$ (FIGURE 26D). The proportion of CD86+ dendritic cells was slightly increased after two days ( $p>0.05)$ and significantly increased after three days of LAQ treatment $(p<0.05)$ compared to controls (FIGURE 26F). TABLE 24 summarizes sample sizes, mean frequencies of total splenic dendritic cells as well as mean proportions of CD80+ and CD86+ dendritic cells with corresponding SEM and p-values.

Previously published data pointed towards an indirect interference of LAQ with T cells, mediated by a direct modulation of antigen-presenting cells, which could suggest delayed effects of LAQ on T cells compared to dendritic cells. Thus, a treatment interval of four days was chosen to analyze early effects of LAQ on T cells in this study. Since LAQ-mediated alterations of naïve and effector memory T cells (see 3.1.2 and FIGURE 3) as well as pro-inflammatory Th1 and Th17 cells (see 3.1.3 and FIGURES 4 and 5) were observed after eleven days of treatment in this work, the frequencies of naïve T cells (FIGURE 27A), effector memory T cells (FIGURE 27A), IL-17+ (FIGURE 27D) and IFNY+ (FIGURE 27F) CD4+ T cells were used as readouts for effective LAQ treatment after four days.

The effects of LAQ on T cells were indeed delayed compared to NK cells and dendritic cells. After four days of LAQ treatment no considerable changes were observed in any of the parameters assessed. The mean frequencies of $C D 62 L+C D 44^{\text {low }}$ naïve T cells were similar in LAQtreated animals (mean $68.8 \% \pm 2.6 \%$ ) and controls (mean $74.6 \% \pm 1.2 \%, p>0.05$ ) (FIGURE 27B). Further, four days of LAQ treatment did not induce a decrease of CD62L- CD44 ${ }^{\text {high }}$ effector memory $T$ cells (Ctrl: mean 13.1\% $\pm 0.9 \%$; LAQ: mean $17.6 \% \pm 2.6 \%$; $p>0.05$ ) (FIGURE 27C), as observed after eleven days of treatment (compare 3.1.2 and FIGURE 3C). Th17 cells were barely detectable at day four after immunization and LAQ treatment and their frequency did not significantly differ between the LAQ (mean $0.04 \% \pm 0.03 \%$ ) and control group (mean $0.05 \% \pm$ 
$0.01 \%, p>0.05$ ) (FIGURE 27E). The frequencies of Th1 cells were also very low after four days of LAQ treatment and similar in LAQ-treated (mean $0.3 \% \pm 0.04 \%$ ) and control mice (mean $0.2 \% \pm$ 0.05\%) (FIGURE 27G).

TABLE 24: In vivo LAQ treatment reduces the total frequency of splenic dendritic cells within three days and upregulates the co-stimulatory molecules CD80 and CD86 within two to three days

\section{Percentage of total splenic dendritic cells}

\begin{tabular}{cccc} 
Control & LAQ & \\
Days of treatment & $(\mathrm{n}=6)$ & $(\mathrm{n}=6)$ & $>$-value \\
\hline 1 & $1.7 \% \pm 0.2 \%$ & $1.9 \% \pm 0.3 \%$ & $>0.05$ \\
2 & $1.0 \% \pm 0.1 \%$ & $1.0 \% \pm 0.1 \%$ & $<0.01$ \\
& $0.7 \% \pm 0.05 \%$ & $0.5 \% \pm 0.05 \%$ & \\
\end{tabular}

Control

\begin{tabular}{cccc} 
& Control & LAQ & \\
Days of treatment & $(\mathrm{n}=3)$ & $(\mathrm{n}=3)$ & p-value \\
\hline 1 & $33.5 \% \pm 1.7 \%$ & $35.9 \% \pm 1.8 \%$ & $>0.05$ \\
2 & $19.9 \% \pm 0.9 \%$ & $34.2 \% \pm 3.2 \%$ & $<0.05$ \\
3 & $21.6 \% \pm 1.0 \%$ & $36.4 \% \pm 1.5 \%$ & $<0.01$ \\
\hline Days of treatment & Percentage CD86+dendritic cells & \\
\hline 1 & Control & LAQ & p-value \\
\hline 2 & $(\mathrm{n}=3)$ & $(\mathrm{n}=3)$ & $>0.05$ \\
& $17.8 \% \pm 1.8 \%$ & $18.0 \% \pm 2.1 \%$ & $>0.05$ \\
& $14.6 \% \pm 0.3 \%$ & $18.9 \% \pm 1.8 \%$ & $<0.05$ \\
\hline
\end{tabular}

Data are presented as mean \pm SEM. 
A

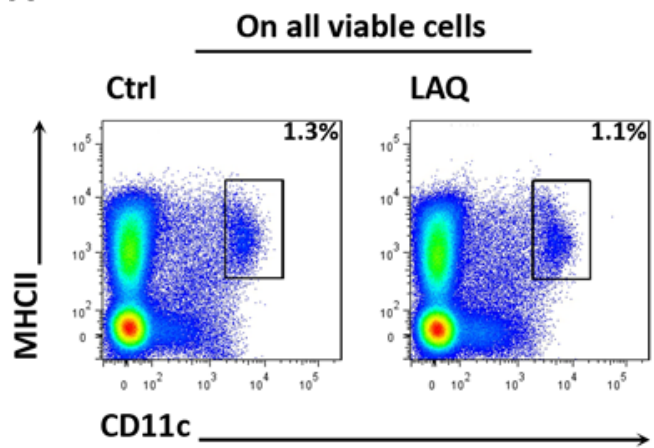

C

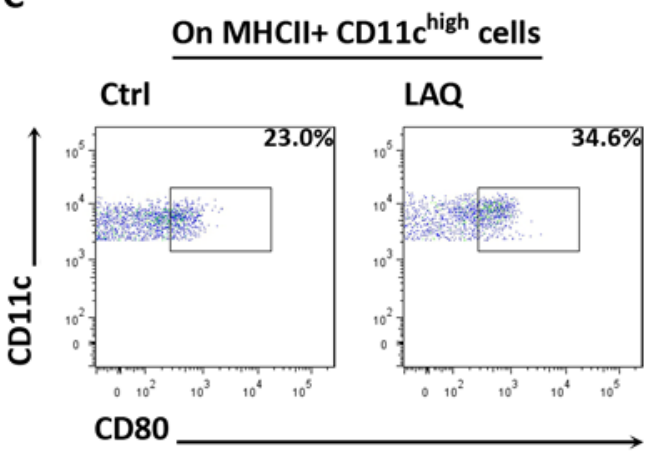

E

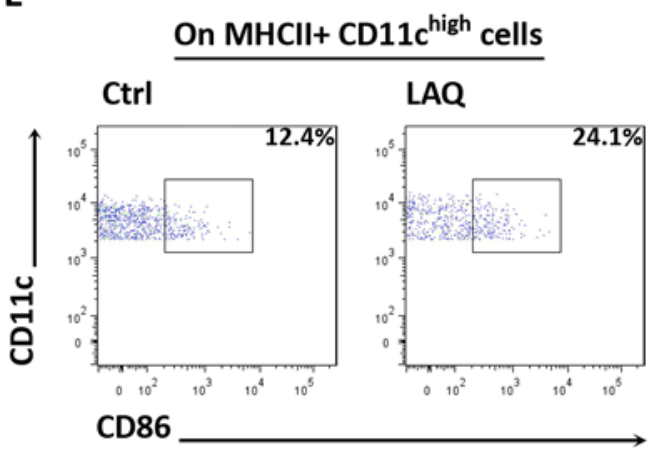

B

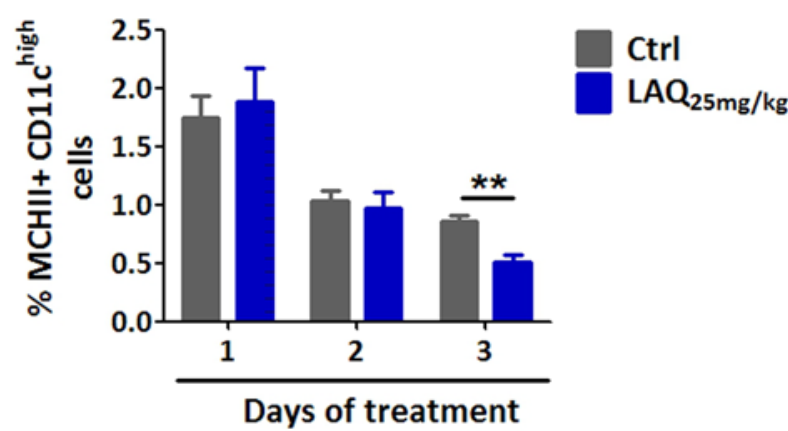

D

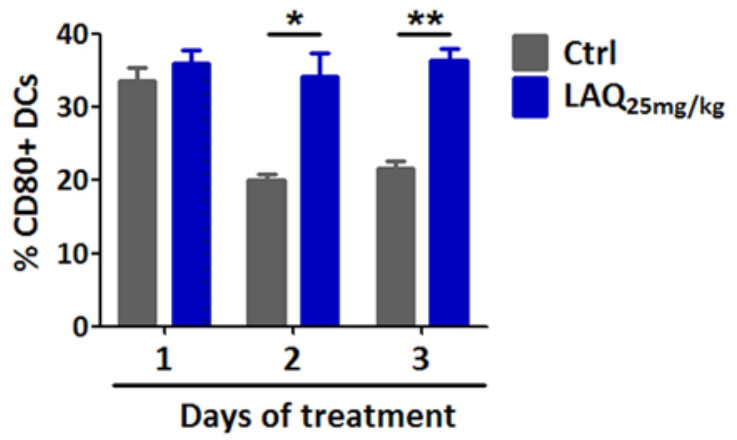

$\mathbf{F}$

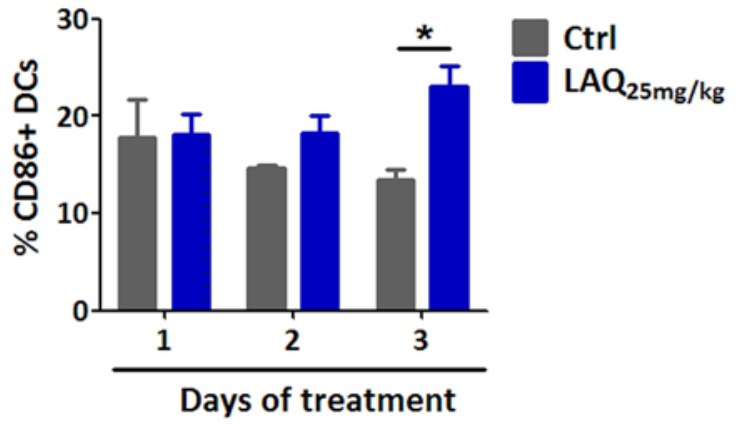

FIGURE 26: LAQ reduces total dendritic cells and leads to upregulation of the co-stimulatory molecules CD80 and CD86 after two to three days of treatment

Time course experiments were performed to study the kinetics of LAQ-effects on dendritic cells. Splenocytes were isolated from animals immunized with $M O_{35-55}$ and pre-treated with $L A Q(25 \mathrm{mg} / \mathrm{kg})$ (blue) or vehicle (gray) for one, two or three days in vivo. Flow cytometry was used to analyze the frequency of splenic dendritic cells $(\mathbf{A}, \mathbf{B})$, as well as the proportions of $\mathrm{CD} 80+(\mathrm{C}, \mathrm{D})$ and $\mathrm{CD} 86+(\mathrm{E}, \mathrm{F})$ dendritic cells. Two days treatment with $L A Q$ are sufficient to significantly increase the proportion of CD80+ dendritic cells compared to controls ( $n=3$ per group, ${ }^{*} p<0.05,{ }^{* *} p<0.01$, unpaired t-test, one representative experiment) (D). Three days of LAQ treatment are necessary to significantly augment the proportion of CD86+ dendritic cells ( $\mathrm{n}=3$ per group, * $\mathrm{p}<0.05$, unpaired t-test, one representative experiment) $(\mathbf{F})$ and to reduce the frequency of total splenic dendritic cells ( $n=6$ per group, ${ }^{* *} p<0.01$, Mann-Whitney $U$ test) (B). Data are presented as mean \pm SEM. 
A

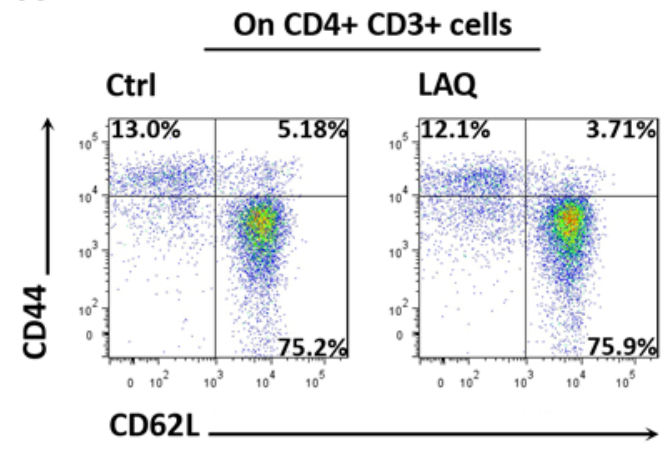

D

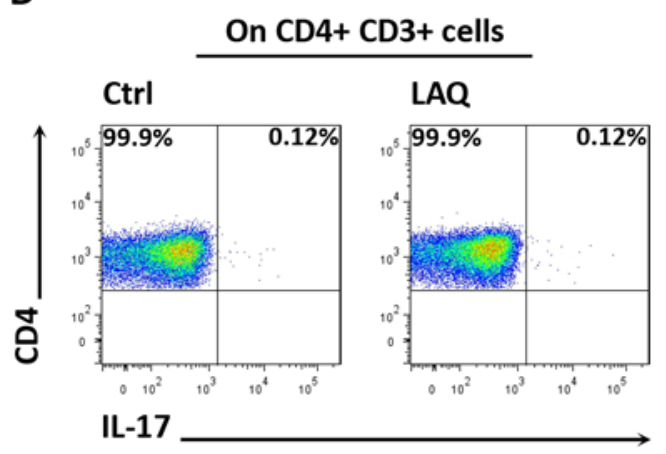

$\mathbf{F}$

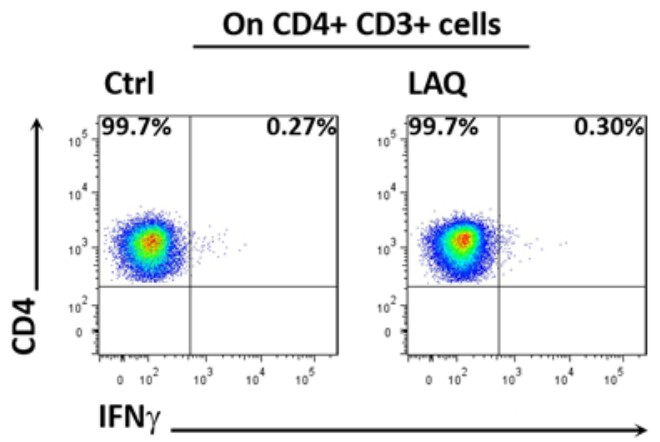

B

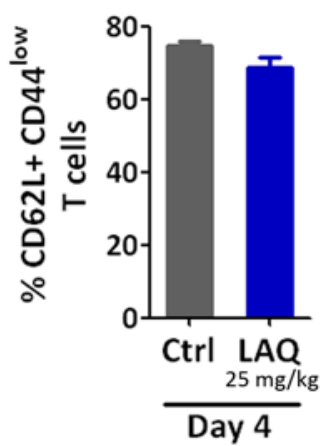

E

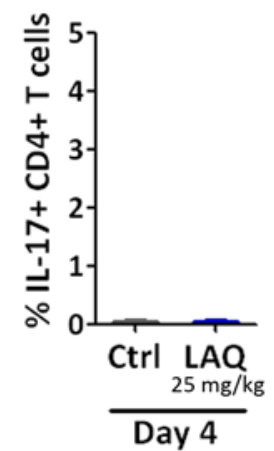

G

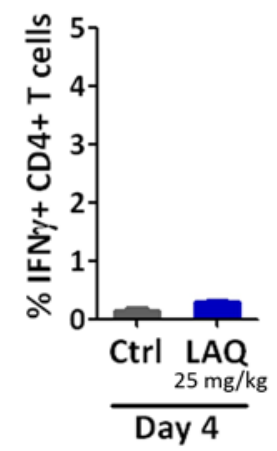

FIGURE 27: No effects of LAQ on T cells after four days of treatment

To study early effects of $L A Q$ on $T$ cells, splenocytes were isolated from $M O_{35-55}$-immunized animals which had received treatment with $25 \mathrm{mg} / \mathrm{kg}$ LAQ (blue) or vehicle (gray) for four days in vivo. The frequencies of CD62L+CD44 ${ }^{\text {low }}$ naïve T cells (A, B), CD62L- CD44 high effector memory T cells (A, C), Th17 cells (D, E) and Th1 cells $(\mathbf{F}, \mathbf{G})$ were evaluated by flow cytometry. Four days of LAQ treatment are not sufficient to induce any significant changes in T cells. The proportions of naïve T cells ( $n=3$ per group, $p>0.05$, unpaired t-test) (B) and effector memory T cells ( $n=3$ per group, $p>0.05$, unpaired t-test) (C) do not significantly differ between LAQ- and vehicle-treated animals. The frequencies of Th17 cells ( $n=3$ per group, $p>0.05$, unpaired t-test) (E) and Th1 cells ( $p>0.05$, unpaired t-test) (G) are equally low in LAQ-treated and control samples. Data are presented as mean \pm SEM. 


\subsubsection{The effect of LAQ on NK cells was independent of T and B cells in vivo}

LAQ induced changes in NK cells as well as T cells after eleven days of treatment (compare 3.1 and 3.3). As mentioned in the previous chapter time course experiments revealed an earlier onset of effects of LAQ in NK cells compared to T cells (compare 3.5.1 and FIGURES 25 and 27). To confirm that LAQ-mediated effects on NK cells were independent of T and B cells in vivo, the next set of experiments investigated naïve Rag1-deficient mice, which are devoid of $T$ and $B$ cells (Mombaerts et al., 1992). As previously shown in this work, the response of NK cells to LAQ therapy was similar in naïve and immunized mice, indicated by a comparable shift towards more CD27+ NK cells (compare 3.3.2.1 and FIGURE 11) and increased expression of the activation marker CD69 (compare 3.3.2.2.1 and FIGURE 12). Thus, naïve Rag1-deficient mice are a suitable model to study the role of T and B cells for the effects of LAQ on NK cells. Rag1-deficient mice were treated with either $25 \mathrm{mg} / \mathrm{kg}$ LAQ or vehicle for 20 days. Changes in subpopulations and the activation of NK cells were evaluated, whereby the surface markers CD27, CD11b (FIGURE 28A) and CD69 (FIGURE 28D) were assessed on NK1.1+ CD3- cells by flow cytometry.

The effect of LAQ on NK cells was independent of T and B cells in vivo. LAQ-induced changes in NK cells of Rag $1^{-/}$mice were comparable to those observed in immunized wild type mice (compare 3.3.2.1 and FIGURE 11B).

LAQ treatment induced a shift in NK cell subpopulations towards more CD27+ and fewer CD11b+ NK cells in Rag1 ${ }^{--}$mice. LAQ significantly increased the mean frequency of CD27+ NK cells from $11.2 \% \pm 3.1 \%$ in controls to $35.8 \% \pm 2.0 \%$ in treated animals $(p<0.01)$ (FIGURE $28 \mathrm{~B}$ ), which corresponds to a three-fold increase. The mean frequency of CD11b+ NK cells was nearly halved in LAQ-treated animals (mean $28.5 \% \pm 3.8 \%$ ) compared to controls (mean $52.4 \% \pm 6.7 \%, p<0.05$ ) (FIGURE 28C).

LAQ therapy significantly augmented the mean frequency of CD69+ NK cells from $33.7 \% \pm$ $5.2 \%$ in controls to $71.8 \% \pm 8.8 \%$ in treated Rag $1^{--}$animals $(p<0.01)$ (FIGURE $28 E$ ).

\subsubsection{NK cell depletion did not affect the dendritic cell phenotype after LAQ treatment in vivo}

The kinetic of LAQ-induced changes was similar for NK cells and dendritic cells (compare 3.5.1 and FIGURES 25 and 26). Additional experiments investigated whether the effects of LAQ on NK cells and dendritic cells were independent. As previously described in this work, LAQ treatment reduced the frequencies of total splenic dendritic cells and myeloid dendritic cells (compare 3.2 and FIGURES 8 and 9). The next set of experiments assessed these two parameters in NK cell-competent and NK cell-depleted animals. 
A

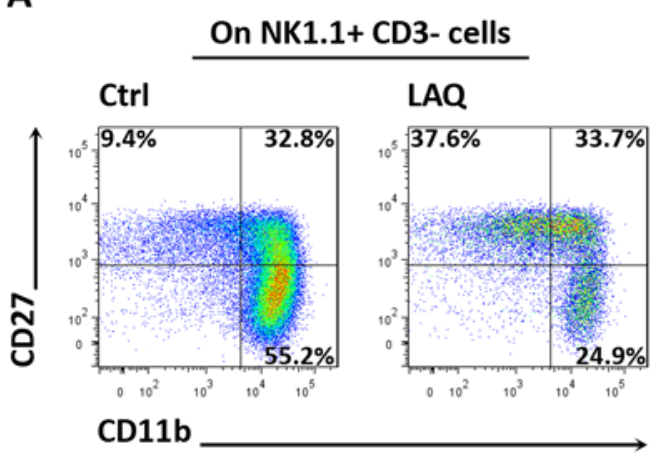

D

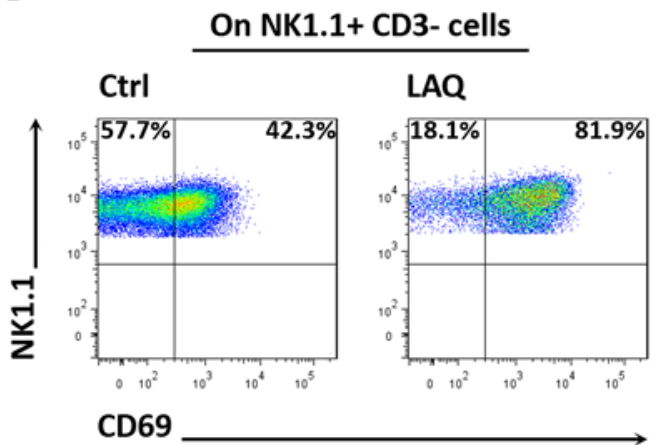

B

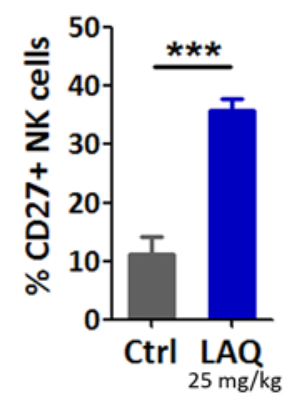

E

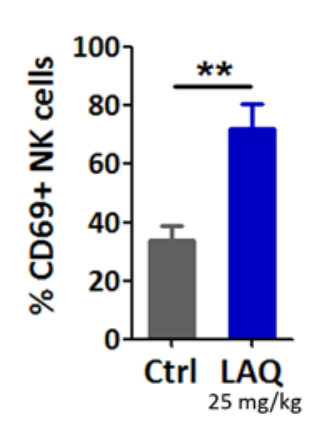

C

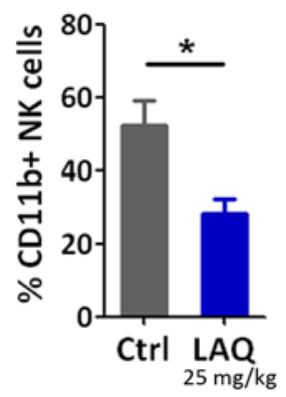

FIGURE 28: T and B cells are dispensable for the LAQ effect on NK cells

To confirm that the effects of $L A Q$ are independent of $T$ and $B$ cells, NK cell changes under LAQ were investigated in Rag $1^{\%-}$ mice. Splenocytes were isolated from Rag $1^{\%-}$ mice pre-treated with $25 \mathrm{mg} / \mathrm{kg} \mathrm{LAQ}$ (blue) or vehicle (gray) for 20 days in vivo. The effect of LAQ on NK cell subpopulations (A, B, C) and activation (D, E) were analyzed by flow cytometry, measuring the surface expression of CD27, CD11b and CD69 on NK1.1+ NK cells. LAQ treatment significantly increases the proportion of CD27+ NK cells $\left({ }^{* * *} p<0.001\right.$, unpaired t-test) (B) and reciprocally decreases the proportion of CD11b+NK cells (* $p<0.05$, unpaired t-test) (C) in Rag1 ${ }^{-/}$mice (Ctrl: $\left.n=3, L A Q: n=4\right)$. The frequency of CD69+ NK cells is approximately doubled in LAQ-treated $\operatorname{Rag}^{-1-}(n=4)$ mice compared to controls $(n=3, * * p<0.01$, unpaired t-test) (E). Data are presented as mean \pm SEM.

NK cells were depleted in C57BI/6J mice by intraperitoneal administration of $300 \mu \mathrm{g}$ antiNK1.1 antibody (clone PK-136) per animal at the two days prior to immunization and then every other day until splenocytes were harvested. NK cell-competent animals received identical treatment with the isotype control antibody C1.18.4. Successful NK cell depletion was controlled in the peripheral blood before immunization by flow cytometry, which revealed highly efficient depletion of NK cells (above 99\%) in all PK-136-treated animals. Daily administration of either 25 $\mathrm{mg} / \mathrm{kg}$ LAQ or vehicle to NK cell-competent and NK cell-depleted mice was started at the day of immunization with $\mathrm{MOG}_{35-55}$. After eleven days of $L A Q$ treatment, splenic dendritic cells were 
isolated and effects of LAQ were evaluated by flow cytometry. Data were analyzed as total frequency of MHCII+CD11 $c^{\text {high }}$ cells (FIGURE 29A) and as frequency of CD11b+ cells among the $\mathrm{MHCll}+\mathrm{CD} 11 \mathrm{C}^{\text {high }}$ cells (FIGURE 29C).

LAQ affected dendritic cells independently of NK cells in vivo. The total frequency of splenic dendritic cells was significantly reduced in LAQ-treated NK cell-competent mice (mean $0.6 \% \pm 0.1 \%$ ) compared to their controls (NK cell-competent mice without LAQ: mean $1.6 \% \pm$ $0.2 \%, p<0.01$ ). A similar reduction was observed in NK cell-depleted mice treated with LAQ (mean $0.7 \% \pm 0.1 \%$ ) compared to these controls (mean $1.5 \% \pm 0.2 \%, p<0.05$ ) (FIGURE 29B).

The mean frequencies of $C D 11 b+$ myeloid dendritic cells were also decreased upon $L A Q$ treatment irrespective of a prior NK cell depletion. In NK cell-competent mice, LAQ therapy reduced the mean frequency of $\mathrm{CD} 11 \mathrm{~b}+$ myeloid dendritic cells from $72.1 \% \pm 1.5 \%$ in controls to $52.3 \% \pm 0.2 \%$ in treated animals $(p<0.001)$. An analogous effect was observed in NK cell-depleted animals, where LAQ induced a drop in the mean frequency of CD11b+ myeloid dendritic cells from $71.2 \% \pm 1.5 \%$ in controls to $50.2 \% \pm 1.1 \%$ in treated animals $(p<0.001)$ (FIGURE 29D).

\subsubsection{LAQ directly activated NK cells in vitro}

LAQ treatment influenced dendritic cells independently of NK cells in vivo (compare 3.5.3). To elucidate whether the LAQ-mediated changes in NK cells were also independent of dendritic cells, NK cells were isolated from naïve mice and treated with 0 or $10 \mu \mathrm{M} L A Q$ in vitro. The frequency of CD69+ NK cells in samples with and without LAQ was analyzed by flow cytometry (FIGURE 30A).

LAQ directly increased the frequency of CD69+ NK cells in vitro. CD69+ NK cells were augmented from $64.8 \% \pm 0.9 \%$ in control samples to $72 \% \pm 1.1 \%$ in LAQ-treated samples $(p<0.05)$ (FIGURE 30B).

To investigate whether bone marrow-derived dendritic cells, pre-treated with $L A Q$, could mimic direct effects of LAQ on the activation of NK cells, NK cells were either cultured alone or cocultured with bone marrow-derived dendritic cells, pre-treated with 0 or $10 \mu \mathrm{M} \mathrm{LAQ}$.

No significant increase of CD69+ NK cells was observed upon co-culture of NK cells with bone marrow-derived dendritic cells, irrespective of LAQ pre-treatment. The mean frequencies of CD69+ NK cells were similar when NK cells were cultured alone (mean $67.3 \% \pm 1.0 \%$ ), co-cultured with naïve bone marrow-derived dendritic cells (mean $59.9 \% \pm 2.5 \%$ ) or co-cultured with pretreated bone marrow-derived dendritic cells (mean $67.7 \% \pm 3.1 \%$ ) (FIGURE 30C). 
A

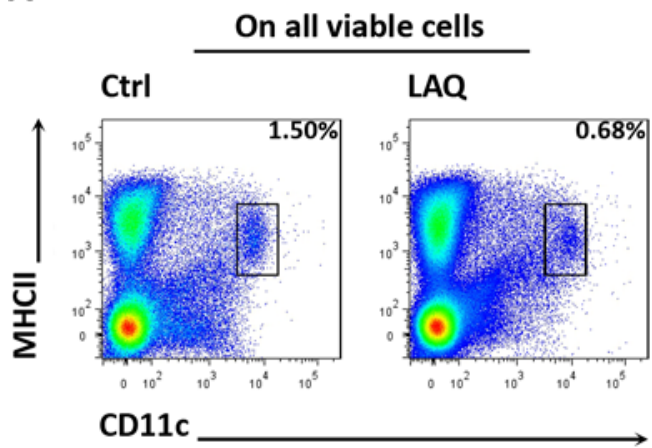

C

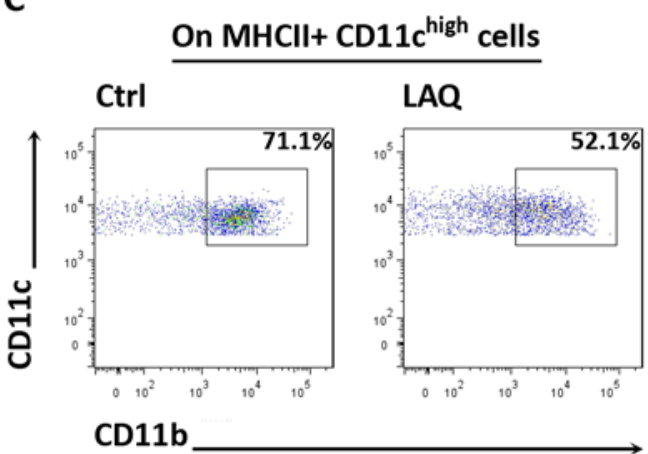

B

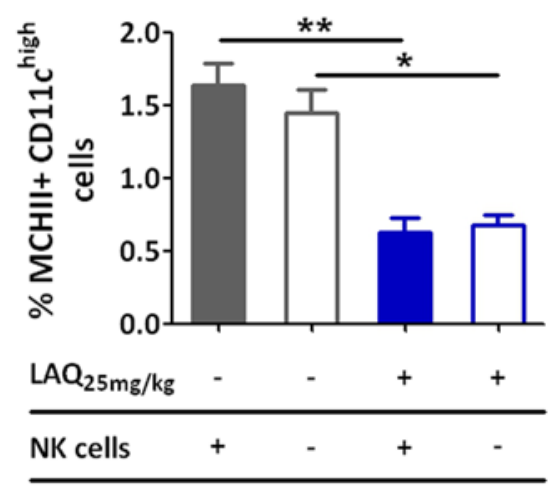

D

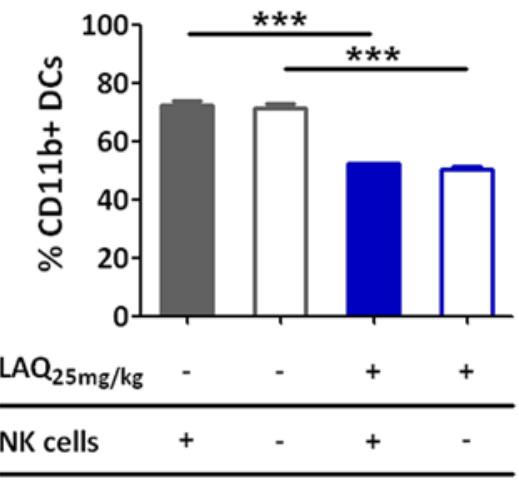

FIGURE 29: The effects of LAQ on dendritic cells are not mediated by NK cells

To analyze whether LAQ-induced changes of dendritic cells are dependent on the presence of NK cells, NK cells were depleted prior to immunization using an anti-NK1.1 antibody. After successful NK cell depletion, mice were immunized with $\mathrm{MOG}_{35-55}$ and treated with $25 \mathrm{mg} / \mathrm{kg}$ LAQ (blue) or vehicle (gray) for eleven days in vivo. LAQ-induced changes in splenic dendritic cell frequencies (A, B) and the proportion of myeloid dendritic cells (C, D) were analyzed by flow cytometry. LAQ alters dendritic cells directly without NK cell involvement. LAQ reduces the frequency of splenic dendritic cells in NK cell-depleted mice to a similar extent as in NK cell-competent mice. The LAQ-dependent reduction is statistically significant in both experimental groups ( $n=3$ per group, * $p<0.05, * * p<0.01$, one way ANOVA with Bonferroni post test) (B). The LAQ-induced decrease of myeloid dendritic cells is comparable in NK cell-depleted and NK cellcompetent mice and the effect is statistically significant in both groups ( $n=3$ per group, *** $p<0.001$, one way ANOVA with Bonferroni post test) (D). Data are presented as mean \pm SEM. 
A

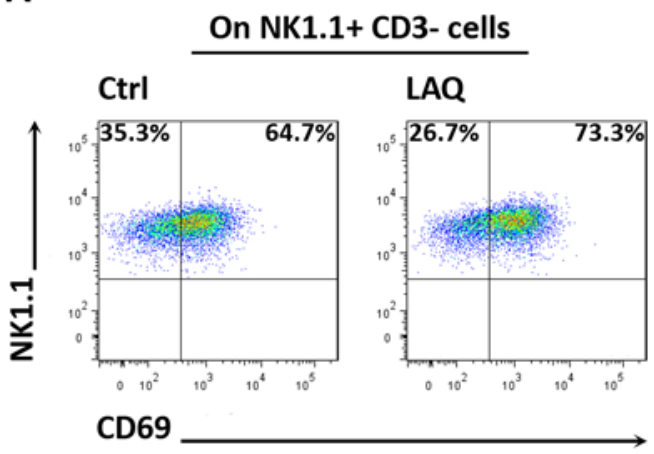

C

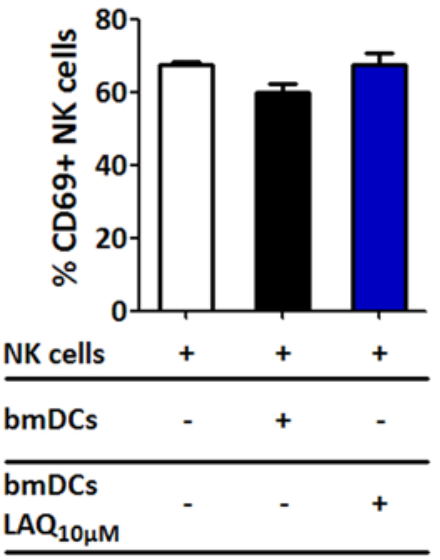

B

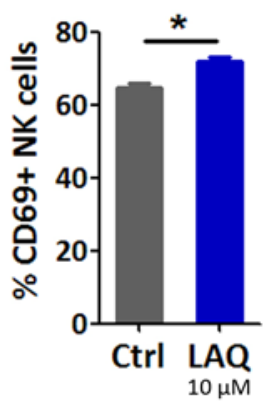

derived dendritic cells pre-treated with 0 or $10 \mu \mathrm{M}$ LAQ. To assess the effects of LAQ the increase of CD69+ NK cells was measured by flow cytometry (A). Direct treatment with $10 \mu \mathrm{M}$ LAQ in vitro significantly raises the expression of CD69 on NK cells ( $n=4$ per group, * $\mathrm{p}<0.05$, Mann-Whitney test, one representative experiment) (B). Co-culture with pre-

treated bone marrow-derived dendritic cells does not mimic direct LAQ treatment. The frequencies of CD69+ NK cells are similar in samples without dendritic cells and in samples incubated with dendritic cells with and without LAQ pre-treatment ( $n=10$ per group, $p>0.05$, Kruskal-Wallis test with Dunn's post test) (C). Data are presented as mean \pm SEM.

In summary, the presented experiments revealed effects of LAQ on NK cells and dendritic cells within the first three days of treatment, whereas T cells had not yet responded to LAQ after four days of therapy. The effects of LAQ on dendritic cells were independent of NK cells, as evidenced by a similar reduction of dendritic cells in NK cell-competent and NK cell-depleted mice after LAQ administration. In turn, NK cells were directly activated by LAQ in vitro in the absence of any other cell type and co-culture with pre-treated dendritic cells could not induce this effect. 


\subsection{NK cell depletion reduced the therapeutic efficiency of LAQ in EAE}

Preventive LAQ treatment completely inhibited EAE induction in C57BI/6J mice (compare 3.1.1) and directly modified NK cell properties (compare 3.3, 3.4.1.1 and 3.5.4). Therefore, the final set of experiments investigated the impact of NK cells on the therapeutic efficiency of LAQ in EAE.

These experiments were performed using Th/+ mice, which possess MOG-specific B cells and pathogenic antibodies (Litzenburger et al., 1998). After immunization with $\mathrm{MOG}_{35-55}$, EAE is very efficiently induced in Th/+ mice without therapeutic intervention. The experimental setup is summarized in FIGURE 31. Briefly, NK cells were initially depleted in Th/+ mice by the administration of $300 \mu \mathrm{g}$ anti-NK1.1 antibody PK-136 per animal at day one and two before immunization. To sustain NK cell depletion, anti-NK1.1 antibody was then applied every other day until the end of the experiment (day 28 p.i.). NK cell-competent Th/+ mice received the corresponding treatment with the isotype control antibody C1.18.4. At the day of immunization with $\mathrm{MOG}_{35-55}$ NK cell-competent and NK cell-depleted Th/+ mice were randomized into the different treatment groups, which received either $25 \mathrm{mg} / \mathrm{kg}$ LAQ or vehicle. Daily treatment with LAQ or vehicle was started at the day of immunization and carried out until the end of the experiment. The disease scores were assessed daily in each animal from the day of immunization until day 28 after immunization. Finally, this experimental setup compared four different groups:

1. Th/+ mice treated with vehicle and isotype control antibody: "control"

2. Th/+ mice treated with vehicle and anti-NK1.1 antibody: "control ${ }_{\text {NK cell-depleted" }}$

3. Th/+ mice treated with LAQ and isotype control antibody: "LAQ"

4. Th/+ mice treated with LAQ and anti-NK1.1 antibody: "LAQ ${ }_{\text {NK cell-depleted" }}$

Disease severity and mortality were compared between these different experimental groups to assess the importance of NK cells for the therapeutic effect of LAQ in EAE. 
Immunization with $\mathrm{MOG}_{35-55}$
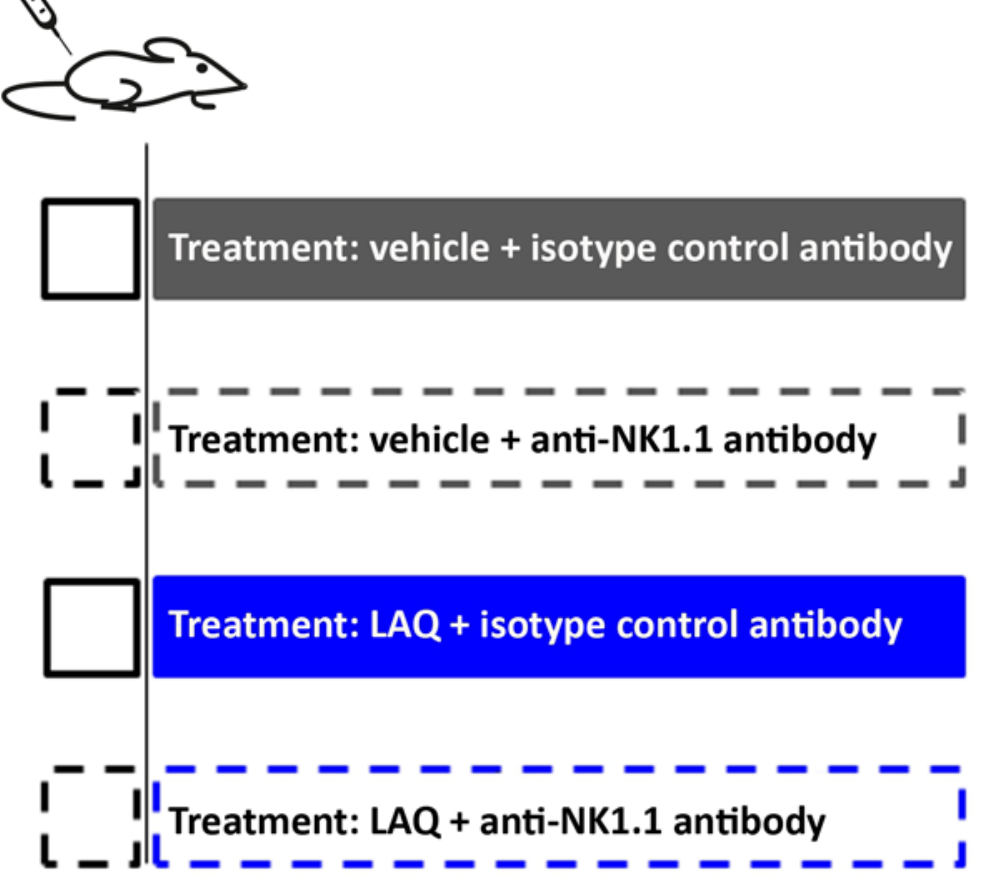

\section{Experimental \\ groups}

control

control $_{\mathrm{NK} \text { cell-depleted }}$

LAQ

$\mathrm{LAQ}_{\mathrm{NK} \text { cell-depleted }}$

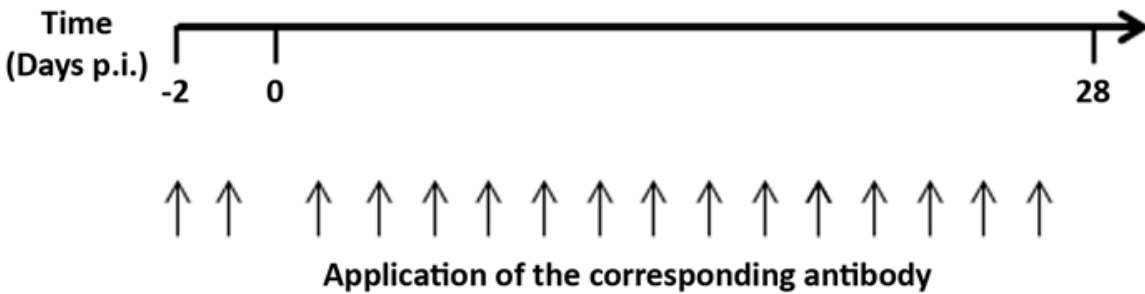

FIGURE 31: Experimental setup for NK cell depletion and LAQ treatment in immunized Th/+ mice

The aim of this experimental setup is to investigate the impact of NK cells on the therapeutic efficiency of LAQ in EAE. Therefore, NK cells are initially depleted in naïve Th/+ mice at day two and one before immunization by intraperitoneal injection of the anti-NK1.1 antibody PK-136 (dashed lines). Subsequent administration of the anti-NK1.1 antibody every other day until day 28 p.i. inhibits the recovery of NK cells. NK cell-competent Th/+ mice receive identical treatment with the isotype control antibody C1.18.4 (solid lines). At the day of immunization with $\mathrm{MOG}_{35-55}$, NK cell-competent and NK cell-depleted animals are subjected to different treatment groups, receiving either $25 \mathrm{mg} / \mathrm{kg}$ LAQ (blue) or vehicle (gray). LAQ and vehicle are administered daily and orally from the day of immunization until the end of the experiment at day 28 after immunization. According to the administration of NK cell-depleting antibody or isotype control and the therapy with LAQ or vehicle, this experimental setup generates four different experimental groups: control, control ${ }_{\mathrm{NK} \text { cell-depleted }} \mathrm{LAQ}$ and $\mathrm{LAQ}_{\mathrm{NK} \text { cell-depleted }}$. 


\subsubsection{NK cell depletion was efficient in Th/+ mice}

The efficiency of the NK cell depletion was controlled in each animal directly before immunization and at day 28 after immunization, by analyzing the presence of NK1.1+ NKp46+ cells in the blood by flow cytometry (FIGURE 32). NK cells were present in animals treated with the isotype control antibody and absent in all animals treated with the NK cell depleting antibody at both time points (FIGURE 32).

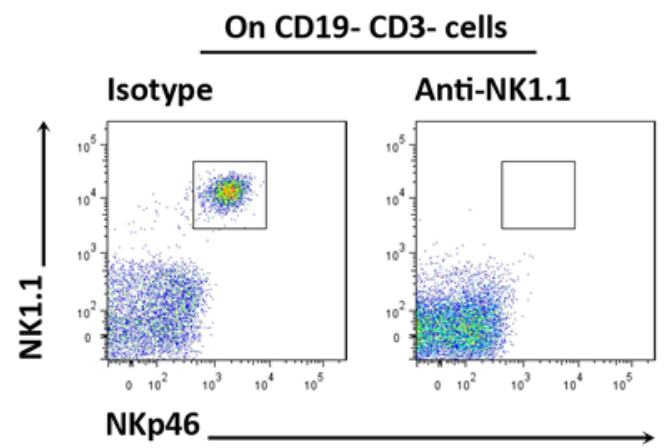

FIGURE 32: NK cell depletion is efficient

NK cells were initially depleted by intraperitoneal administration of an anti-NK1.1 antibody one and two days prior to immunization and NK cell depletion was sustained by subsequent application of the antibody every other day until the end of the experiment. The efficiency of NK cell depletion was assessed directly before immunization and at day 28 after immunization. The presence or absence of NK1.1+ NKp46+ NK cells in peripheral blood was analyzed by flow cytometry. NK cells are absent in all depleted animals and present in tested animals treated with the isotype control antibody.

\subsubsection{NK cell depletion exacerbated EAE symptoms in LAQ-treated animals}

To investigate the importance of NK cells for the therapeutic efficiency of LAQ in EAE, the disease severity was assessed in the four experimental groups (control, control $_{\mathrm{NK}}$ cell-depleted, $\mathrm{LAQ}$ and $L_{A Q_{N K} \text { cell-depleted }}$ ) by surveying clinical scores every day from day 1 until day 28 after immunization. 
The overall comparison of all four experimental groups revealed a marked reduction of clinical severity after LAQ treatment in NK cell-competent animals (FIGURE 33). This beneficial effect of LAQ treatment was markedly attenuated upon NK cell depletion. This group showed a temporal delay of the peak of disease in comparison to vehicle-treated control groups and a similar disease severity at the end of the experiment (FIGURE 33). NK cell depletion had no obvious effect on EAE severity in the vehicle-treated groups, as demonstrated by comparable EAE scores in the control group and the control $\mathrm{NK}_{\text {cell-depleted }}$ group (FIGURE 33).

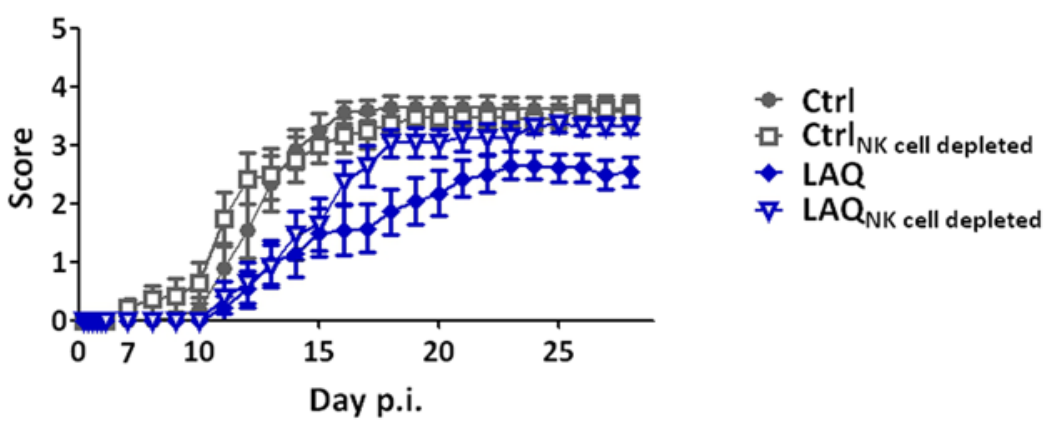

FIGURE 33: LAQ-mediated reduction of EAE severity is dependent on NK cells To study the importance of NK cells for the therapeutic efficiency of LAQ in EAE, NK cell-competent (filled symbols) and NK cell-depleted (empty symbols) $\mathrm{Th} /+$ mice were immunized with $\mathrm{MOG}_{35-55}$ and treated with $25 \mathrm{mg} / \mathrm{kg}$ LAQ (blue) or vehicle (gray) from day 0 until day 28 after immunization. Clinical scores were assessed daily. The overall comparison of all four experimental groups reveals a considerable amelioration of EAE severity upon LAQ treatment in NK cell-competent mice $(n=14)$ compared to controls $(n=13)$. NK cell depletion attenuates the therapeutic LAQ effect, as animals in the $\mathrm{LAQ}_{\mathrm{NK}}$ cell-depleted group $(\mathrm{n}=14)$ display similar disease severity as animals in the control groups. In contrast, NK cell depletion does not alter EAE severity in the vehicle-treated animals, since the EAE courses of control and control $_{\mathrm{NK} \text { cell-depleted }}(\mathrm{n}=14)$ are similar. Data are presented as mean \pm SEM.

To address the crucial question, whether NK cells were essential for the therapeutic efficiency of $L A Q$ in EAE, disease scores from the $L A Q$ group and the $L A Q_{N K}$ cell-depleted group were directly compared in a subanalysis. This subanalysis revealed a pivotal role of NK cells for LAQ efficiency in EAE. NK cell depletion significantly exacerbated the EAE course in LAQ-treated animals $(p<0.001)$ (FIGURE 34). The dependence of the LAQ efficiency on the presence of NK cells 
in EAE was further indicated by increased mean maximal scores in the $L A Q_{N K}$ cell-depleted group (mean $3.5 \pm 0.1$ ) compared to the LAQ group (mean $2.9 \pm 0.2$ ) and considerably higher mean cumulative scores in the $L A Q_{N K}$ cell-depleted group (mean $45 \pm 3.4$ ) compared to the $L A Q$ group (mean $34 \pm 4.2$ ). The detrimental effect of NK cell depletion on LAQ efficiency was less obvious during the early phase of EAE induction, since disease onset was similar in both LAQ-treated groups, but it became evident shortly before the peak of disease and lasted until the end of the experiment (FIGURE 34).

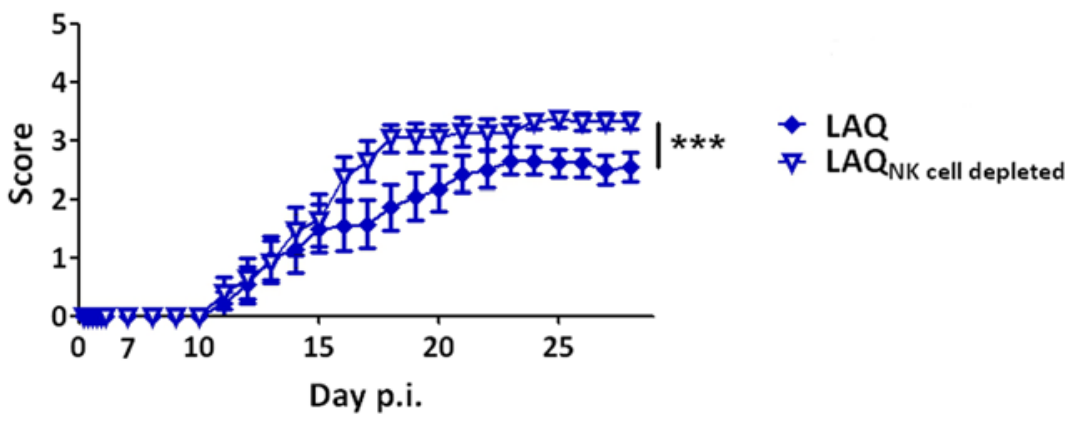

FIGURE 34: NK cell depletion attenuates the therapeutic LAQ effect in EAE

The aim of this experimental part was to study the impact of NK cells on the therapeutic efficiency of LAQ in EAE. Therefore, this subanalysis compared the clinical scores of NK cell-competent (filled symbols) and NK cell-depleted (empty symbols) Th/+ mice immunized with $\mathrm{MOG}_{35-55}$ and treated with 25 $\mathrm{mg} / \mathrm{kg}$ LAQ (blue) from day 0 until day 28 after immunization. This direct comparison shows that NK cells are crucial for the effect of $L A Q$ in this animal model. NK cell depletion significantly exacerbates EAE symptoms in LAQtreated animals ( $n=14$ per group, ${ }^{* * *} p<0.001$ ). The deleterious effect of NK cell depletion on the therapeutic efficiency of LAQ in EAE becomes evident shortly before peak of disease and this difference remains until the end of the experiment. Data are presented as mean \pm SEM. All the statistical analyses of this experiment were performed by Mr. Markus Harden (Department for Medical Statistics, University Medical Center Goettingen) using nonparametric rank-based ANOVA-type statistics. 
Further subanalyses of the NK cell depletion experiment were performed to assess the differences between the remaining groups. Similar to the previously described findings in wild type mice (compare 3.1.1) LAQ treatment was also effective in Th/+ mice. The pairwise comparison revealed a significant reduction of disease severity in the LAQ group compared to the control group $(p<0.001)$ (FIGURE 35A).

To examine whether LAQ treatment was still partially effective after NK cell depletion, the EAE scores of the groups control $\left.\right|_{\mathrm{NK}}$ cell-depleted and $\mathrm{LAQ}_{\mathrm{NK}}$ cell-depleted were directly compared in a subanalysis. Indeed, NK cell depletion did not completely abolish LAQ efficiency. A comparison over the whole experimental time interval of 28 days indicated a significant benefit of LAQ treatment $(p<0.001)$, which was mainly due to a delayed and attenuated onset of disease (FIGURE 35B). However, the long lasting LAQ effect, which was observed in NK cell-competent animals (FIGURE 35A) was not maintained after NK cell depletion. At later time points of the experiment, NK cell-depleted animals treated with LAQ displayed similar disease scores as the corresponding control group (day $18-28$ p.i.) (FIGURE 35B).

The subanalysis of the EAE scores from the two control groups (control and control $\mathrm{NK}_{\text {cell- }}$ depleted) revealed that NK cell depletion had no significant effect on EAE severity in these groups (FIGURE 35C).

Taken together, the presence of NK cells appears to be essential for full and long lasting protective effects of LAQ in EAE.

\subsubsection{NK cell depletion reduced the survival of LAQ-treated animals}

The impact of NK cells on the LAQ efficiency was further investigated by comparing the survival of the four experimental groups (control, control ${ }_{N K}$ cell-depleted, $L A Q$ and $L A Q_{N K}$ cell-depleted) during the complete course of the experiment. Animals were classified as dead if they either died of EAE (score 5) or had to be sacrificed due to ethical reasons (score 4). The Kaplan-Meier curve for all four groups is shown in FIGURE 36.

LAQ treatment efficiently increased the survival of Th/+ mice with EAE. LAQ therapy was fully protective in NK cell-competent animals, mirrored by a survival rate of $100 \%$ at day 28 after immunization. NK cell depletion partially abrogated this beneficial effect, reducing the survival rate to $71.4 \%$ in the $L_{A Q_{N K} \text { cell-depleted }}$ group. Still, NK cell-depleted animals were partly protected, since LAQ treatment halved the risk to die compared to the control groups (hazard ration [control ${ }_{N K}$ cell-depleted versus $L A Q_{N K}$ cell-depleted $]=2.2$ ). The relationship between NK cell depletion and reduced survival rates was specific for LAQ therapy, since NK cell depletion did not alter the 
survival rates in the control groups. At day 28 p.i. the control group and the control $\mathrm{NK}_{\mathrm{NK}}$ cell-depleted group displayed similar survival rates with $46 \%$ and $50 \%$ respectively (FIGURE 36 ).

A

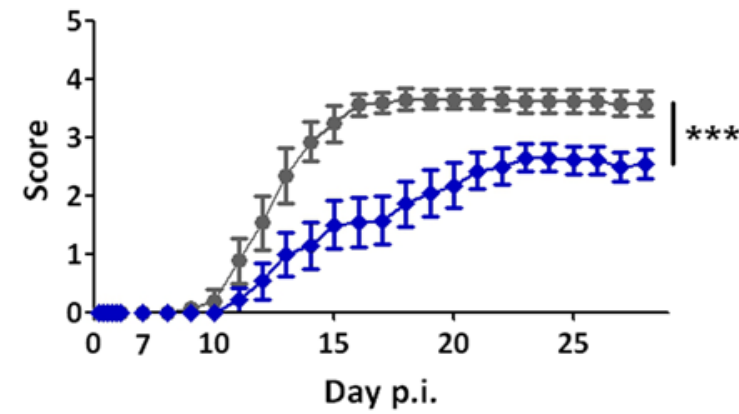

C

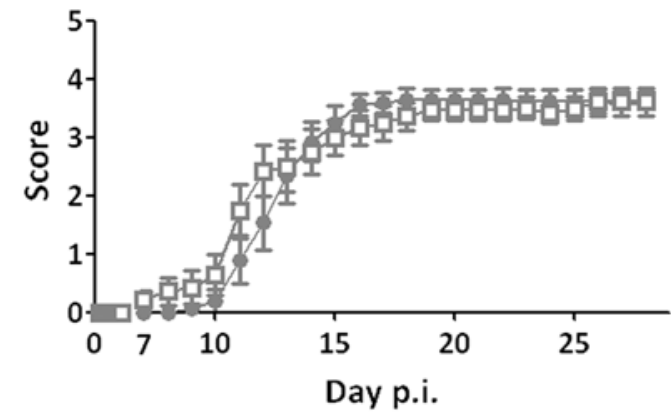

B

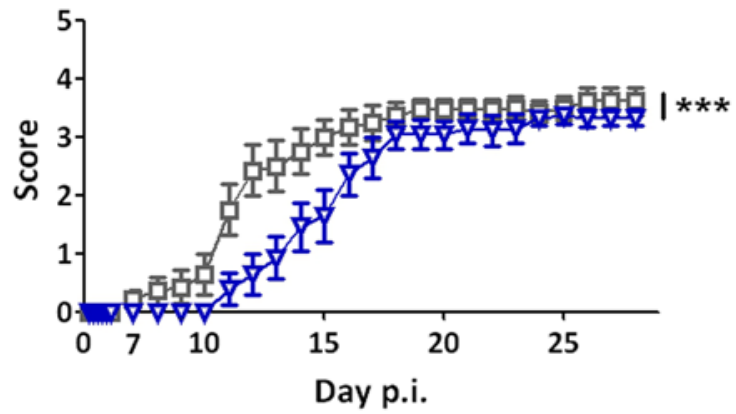

FIGURE 35: LAQ treatment is efficient in Th/+ mice and NK cell depletion does not alter EAE severity in controls

To study the importance of NK cells for the therapeutic efficiency of LAQ in EAE, NK cell-competent (filled symbols) and NK cell-depleted (empty symbols) Th/+ mice were immunized with $\mathrm{MOG}_{35-55}$ and treated with $25 \mathrm{mg} / \mathrm{kg}$ LAQ (blue) or vehicle (gray) from day 0 until day 28 after immunization. Clinical scores were assessed daily. This figure provides different subanalyses of the overall experiment.

LAQ treatment is efficient in NK cell-competent Th/+ mice. The subanalysis of NK cell-competent animals, treated with LAQ or vehicle, reveals significant amelioration of the disease course by LAQ (Ctrl: $n=13$, LAQ: $\left.\mathrm{n}=14,{ }^{* * *} \mathrm{p}<0.001\right)(\mathrm{A})$. Furthermore, LAQ treatment is still partially effective in NK cell-depleted Th/+ mice $(* * * p<0.001)$. The subanalysis of the groups control ${ }_{N K}$ cell-depleted $(n=14)$ and $\mathrm{LAQ}_{N K}$ cell-depleted $(n=14)$ shows delayed and attenuated onset of EAE, but the therapeutic effect is only transient. NK cell-depleted animals reach a similar peak of disease irrespective of $L A Q$ treatment (B). Finally, pairwise comparison of the two control groups reveals that NK cell depletion does not alter EAE severity per se in Th/+ mice (control: $n=13$, control $_{N K}$ cell-depleted $\left.n=14, p>0.05\right)$ (C). Data are presented as mean \pm SEM. All the statistical analyses of this experiment were performed by Mr. Markus Harden (Department for Medical Statistics, University Medical Center Goettingen) using non-parametric rank-based ANOVA-type statistics. 


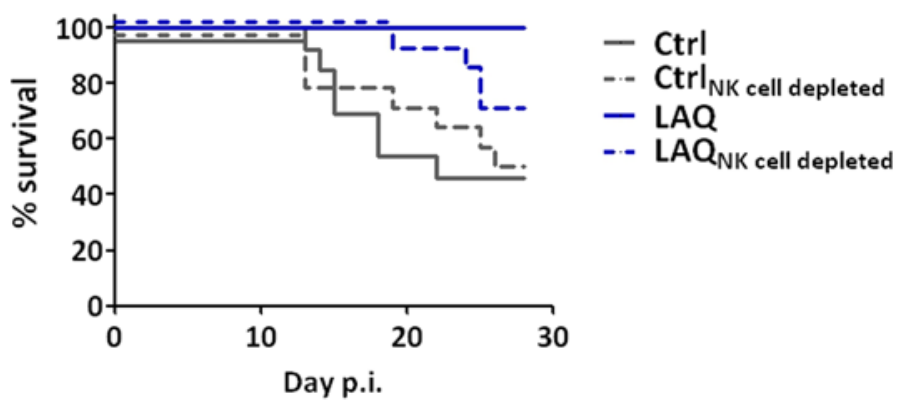

FIGURE 36: NK cell depletion reduces the survival of LAQ-treated mice

The survival during the EAE experiment was assessed using a KaplanMeier curve. LAQ treatment is beneficial in terms of survival. LAQ completely protects NK cell-competent animals from death. This protective effect is partially reduced by the depletion of NK cells, but still the risk to die is halved by LAQ in NK cell-depleted animals compared to depleted controls (hazard ration [control $_{\mathrm{NK}}$ cell-depleted versus $L A Q_{N K}$ cell-depleted $]=2.2$, log-rank test). This effect of NK cell depletion is specific for LAQ, since NK cell depletion does not directly reduce the survival in the control group.

In conclusion, the depletion of NK cells attenuated the protective effect of LAQ in EAE, regarding EAE severity and survival. Previous chapters showed that LAQ treatment was associated with a shift in NK cell subpopulations and an increased activation of NK cells, phenotypically as well as functionally. Taken together, LAQ-mediated changes on NK cells seem to be crucial for its mechanism of action and its therapeutic efficiency in EAE. 


\section{Discussion}

The present study investigated effects of the new immunomodulatory drug LAQ on cells of the innate and adaptive immune system in mice with EAE.

First, this study aimed to analyze the impact of preventive LAQ treatment on EAE induction and pro- and anti-inflammatory $\mathrm{T}$ cell subsets. In $\mathrm{C} 57 \mathrm{BI} / 6 \mathrm{~J}$ mice, $\mathrm{LAQ}$ treatment completely suppressed EAE symptoms and shifted the balance from pro-inflammatory Th1 and Th17 cells to regulatory FoxP3+ T cells in vivo (FIGURE 37). In contrast, in vitro therapy of splenocytes with LAQ did not show any direct effects on IL-17 and IFNY production in T cells.

Further, this work intended to analyze the effect of preventive LAQ treatment on antigen presenting cells by investigating dendritic cells. Data analysis revealed lower frequencies of total dendritic cells and myeloid dendritic cells in the spleen of LAQ-treated animals and LAQ increased the expression of co-stimulatory molecules within the first days of treatment (FIGURE 37).

To characterize additional treatment effects on innate immune cells, LAQ-mediated alterations were studied in NK cells. In the spleen, LAQ reduced total cell numbers as well as absolute NK cell numbers, but NK cell frequencies remained unaltered. NK cell subpopulations were shifted towards more CD27+ and fewer CD11b+ NK cells. Furthermore, LAQ increased NK cell activation by upregulating CD69 and several activating NK cell receptors and by downregulating inhibitory NK cell receptors. Functionally, ex vivo purified NK cells from LAQtreated animals killed tumor cells more efficiently and produced more IFN $\gamma$ than NK cells from vehicle-treated controls (FIGURE 37). In addition, NK cells from LAQ-treated mice led to a more pronounced contact-dependent reduction of T cell proliferation in a co-culture system with T cells and dendritic cells than NK cells from controls.

The work additionally aimed to evaluate direct effects of LAQ on NK cells. The LAQinduced changes in NK cells occurred quickly and independently of T cells in vivo. Notably, LAQ directly activated NK cells in vitro in the absence of any other cell type, whereas co-culture of NK cells with pre-treated bone marrow-derived dendritic cells could not mimic this effect.

The final aim of this thesis was to determine, whether NK cells were essential for the therapeutic efficiency of LAQ in EAE. Experiments in Th/+ mice revealed a central relevance of NK cells for LAQ treatment. LAQ considerably ameliorated EAE symptoms in NK cell-competent mice, but this beneficial effect was profoundly reduced upon NK cell depletion. Survival analysis yielded similar results, whereby all NK cell-competent mice survived under LAQ, but NK cell depletion clearly decreased survival in the LAQ-treated group. 


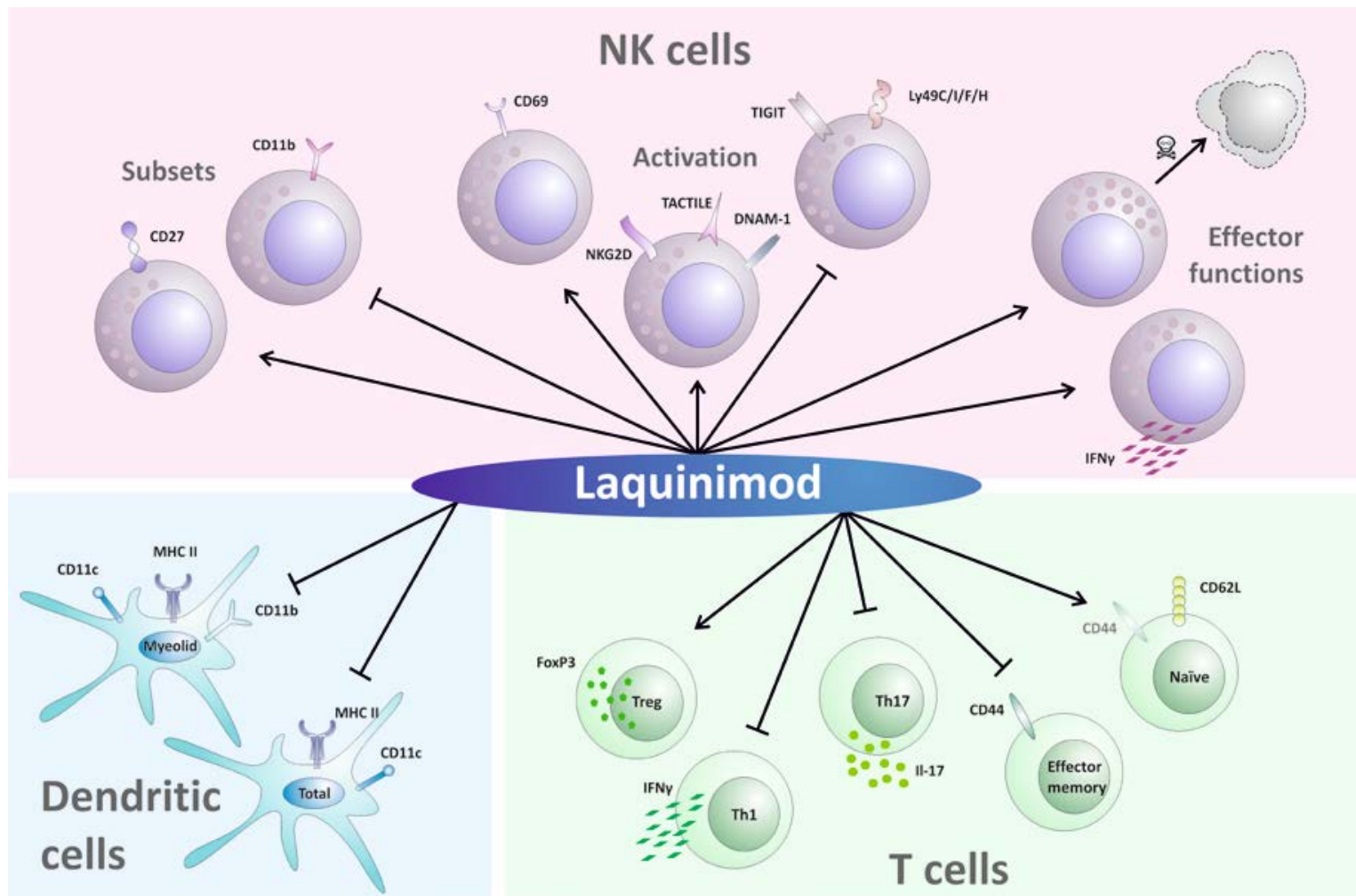

FIGURE 37: Summary of the effects of LAQ treatment on NK cells, dendritic cells and T cells in vivo

LAQ exerts effects on different cells of the innate and the adaptive immune system in vivo. The present work has identified NK cells as new target of LAQ within innate immunity. In vivo LAQ treatment markedly shifts NK cell subpopulations towards more CD27+ and fewer CD11b+ NK cells. Moreover, LAQ increases the activation of NK cells, indicated by the upregulation of the activation marker CD69 and several activating NK cell receptors (TACTILE, DNAM-1, NKG2D) and the reciprocal downregulation of inhibitory NK cell receptors (TIGIT, Ly49C/I/F/H). Functionally, LAQ-treated NK cells display a higher killing efficiency towards tumor cells in vitro and produce more IFN antigen-presenting cells, this thesis reveals a pronounced LAQ-mediated reduction of total dendritic cell and myeloid dendritic cell frequencies in the spleen. With regard to T cells, this work demonstrates a significant increase of naïve T cells and a corresponding decrease of effector memory T cells upon LAQ treatment. Furthermore, LAQ markedly shifts the balance from pro-inflammatory Th1 and Th17 cells towards anti-inflammatory regulatory T cells (Tregs). FIGURE 37 was designed by Mr. Stephan Ott.

\subsection{Preventive LAQ treatment inhibited EAE and reduced dendritic cells and pro-inflammatory $\mathrm{T}$ cells in vivo}

EAE is the most commonly used animal model for $M S$, in which potential immunomodulatory drugs for the treatment of MS are initially tested. First experiments evaluated the efficiency of $L A Q$ on $E A E$ in $C 57 \mathrm{BI} / 6 \mathrm{~J}$ mice immunized with $\mathrm{MOG}_{35-55}$. Preventive treatment 
with $25 \mathrm{mg} / \mathrm{kg}$ LAQ completely abolished EAE incidence, whereas vehicle-treated animals developed clinical EAE symptoms. These data are in line with several published studies. Preventive LAQ treatment has been shown to inhibit EAE induction in a dose dependent manner in mice (Runström et al., 2006, Wegner et al., 2010, Moore et al., 2013, Ruffini et al., 2013) and rats (Yang et al., 2004) previously and was most effective at $25 \mathrm{mg} / \mathrm{kg}$ in mice (Runström et al., 2006, Wegner et al., 2010, Aharoni et al., 2012, Mishra et al., 2012, Schulze-Topphoff et al., 2012, Thöne et al., 2012, Jolivel et al., 2013, Moore et al., 2013).

Recent reports suggest that LAQ might exert its immunomodulatory effects in EAE mainly by decreasing antigen presentation, which impairs the generation of pro-inflammatory myelin protein specific T cells upon immunization and thereby attenuates EAE.

The present work described a LAQ-mediated shift from pro-inflammatory to regulatory T cells. LAQ treatment decreased the frequency of pro-inflammatory Th1 and Th17 cells in the spleen, which was paralleled by reduced secretion of the corresponding cytokines IFNY and IL-17. Regulatory FoxP3+ T cells were in turn increased in the spleen upon LAQ administration. Published studies report analogous LAQ-mediated alterations of T cell subpopulations and proinflammatory cytokines (Wegner et al., 2010, Schulze-Topphoff et al., 2012, Jolivel et al., 2013). These studies further indicate that the observed effects on $T$ cell differentiation appear to be secondary to the LAQ-dependent reduction and modulation of antigen-presentation.

Several additional observations support the notion of an indirect action of LAQ on T cells. LAQ-treated mice display lower frequencies of splenic CD4+ CD11b+ dendritic cells (SchulzeTopphoff et al., 2012). In RRMS, LAQ treatment induces a similar reduction of CD1c+ conventional dendritic cells and CD303+ plasmacytoid dendritic cells in the peripheral blood (Jolivel et al., 2013). The reduced frequencies of antigen-presenting cells could decrease T cell priming per se and LAQ-treated dendritic cells additionally display an anti-inflammatory type II phenotype (Schulze-Topphoff et al., 2012, Thöne et al., 2012). Moreover, the co-stimulatory molecule CD86 is associated with the differentiation of Th2 cells (Kuchroo et al., 1995, Ranger et al., 1996) and has been shown to be upregulated by LAQ (Thöne et al., 2012, Jolivel et al., 2013). In contrast, IL-12, a pivotal cytokine for the differentiation of Th1 cells, is downregulated in LAQ-treated antigenpresenting cells (Schulze-Topphoff et al., 2012). Functionally, LAQ-treated antigen-presenting cells reduce Th1 and Th17 differentiation and antigen-dependent T cell proliferation in vitro, whereas in vivo LAQ treatment of T cells fails to inhibit T cell differentiation in vitro (Schulze-Topphoff et al., 2012, Jolivel et al., 2013). In line with these reports, the present work revealed a pronounced drop of total dendritic cells as well as CD11b+ myeloid dendritic cells in the spleen after LAQ therapy. Temporally, the effects of LAQ on dendritic cells were detected within the first days of treatment in vivo, whereas T cells responded to LAQ therapy with a considerable delay. In vitro treatment of 
splenocytes with LAQ failed to reduce IFN $y$ and IL-17 secretion, which further supports the concept of an indirect action of LAQ on T cells in vivo.

This body of evidence illustrates that LAQ modulates antigen-presenting cells in vivo and indirectly reduces pro-inflammatory $T$ cell differentiation. Th1 as well as Th17 cells play pivotal roles in the pathogenesis of EAE (Steinman, 2007, Stromnes et al., 2008). Therefore, the shift from pro-inflammatory to regulatory $T$ cell subsets could be a crucial part of the mechanism of action of LAQ in EAE and MS.

\subsection{Preventive LAQ treatment induced NK cell activation in vivo}

\subsubsection{LAQ did not alter splenic NK cell frequencies}

LAQ was shown to reduce the frequency of different dendritic cell subsets, revealing an effect of LAQ treatment on innate immune cells. As additional players of innate immunity, NK cells were examined in animals treated with LAQ or vehicle. First, absolute numbers and the frequency of splenic NK cells were determined. Assessment of total splenocytes revealed a $50 \%$ reduction in LAQ-treated compared to vehicle-treated animals. Absolute NK cell numbers were diminished to a similar extent upon LAQ treatment, whereas NK cell frequency remained unaltered.

These data suggest that LAQ inhibits the expansion of splenocytes in $M O_{35-55}$-immunized animals. A likely mechanism for this effect is the reduced frequency of $\mathrm{CD} 11 \mathrm{c}^{\text {high }} \mathrm{MHC}-\mathrm{Il}+$ dendritic cells in LAQ-treated animals (Schulze-Topphoff et al., 2012, Jolivel et al., 2013), which impairs the induction of a primary immune response to MOG. However, other possible mechanisms, like drug-induced apoptosis for instance, cannot be ruled out completely.

Absolute NK cell numbers showed a similar LAQ-mediated decrease as total splenocytes and NK cell frequencies remained stable. Hence, LAQ does not selectively impede NK cell proliferation or induce NK cell apoptosis, but exerts an overall effect on immune cell expansion after immunization.

\subsubsection{LAQ treatment led to a relative expansion of the CD27+ NK cell subset}

To assess LAQ-mediated alterations in different subpopulations of NK cells, splenic NK cells from LAQ- and vehicle-treated animals were phenotyped.

For this purpose, this study evaluated the expression of the surface molecules CD27 and CD11b on NK cells. Subpopulations were classified as follows: CD27+ CD11b- (referred to as CD27+), CD27+CD11b+ and CD27- CD11b+ (referred to as CD11b+) (Chiossone et al., 2009). These three subpopulations differ with regard to their proliferative potential and their expression 
pattern of chemokine and inhibitory receptors (Kim et al., 2002, Hayakawa et al., 2006, Chiossone et al., 2009). For human NK cells, CD27+ cells were shown to be better cytokine producers in response to soluble factors and less cytotoxic against tumor cells than CD11b+ NK cells (Fu et al., 2011). Predominant effector functions of individual subsets of murine NK cells are still more controversial (Takeda et al., 2000, Kim et al., 2002, Hayakawa et al., 2006, Vahlne et al., 2008, Chiossone et al., 2009). LAQ treatment markedly altered the relative frequency of NK cell subpopulations, increasing CD27+ and reciprocally decreasing CD11b+ NK cells, whereas CD27+ CD11b+ NK cells remained unaffected. This LAQ-induced shift in NK cell subpopulations was observed in immunized as well as naïve animals, suggesting a mechanism which is independent of immunological activation.

CD27+ NK cells were shown to further differentiate into CD11b+ NK cells in vivo (Chiossone et al., 2009). As splenic NK cell frequencies were relatively stable under LAQ treatment (as discussed in 4.2.1), the drug-related shift towards more CD27+ NK cells is more likely to be related to a block of NK cell differentiation than to a specific induction of proliferation in this subpopulation.

\subsubsection{LAQ activated NK cells}

Next, the present work investigated the activation state of NK cells after in vivo LAQ treatment on different NK cell subsets.

Ex vivo flow cytometry revealed a considerable activation of NK cells upon LAQ treatment, evidenced by the increased expression of the activation marker CD69. This effect was independent of prior immunization and not restricted to a specific subset of NK cells, since the frequency of CD69+ NK cells was markedly higher among total NK cells as well as among CD27+ and CD11b+ NK cells in response to LAQ treatment.

The LAQ-induced activation of NK cells was accompanied by the specific up- and downregulation of activating and inhibitory receptors, respectively. With regard to activating receptors, LAQ significantly increased the frequencies of TACTILE+, DNAM-1+ and NKG2D+ NK cells. In contrast, the proportions of NK cells expressing the inhibitory receptors TIGIT and Ly49C/I/F/H were reduced upon LAQ treatment. The frequencies of NK cells expressing the activating receptors Ly49D, CD244.2 and LFA-1 and the inhibitory receptors Ly49A and Ly49G2 were similar in LAQ- and vehicle-treated animals. In these experiments only one single activating receptor, $C D 2$, was found to be decreased upon $L A Q$ exposure and there was not any inhibitory receptor increased, among all the investigated receptors.

Taken together, LAQ treatment altered the balance of activating and inhibitory NK cell receptors in a way which should lower the threshold for NK cell activation. 
NK cells express pairwise receptor families, consisting of activating and inhibitory receptors which recognize the same ligands on target cells, to assure precise regulation of NK cell effector functions (Stanietsky et al., 2009, Stanietsky et al., 2013). The activating receptors DNAM-1 and TACTILE together with the inhibitory receptor TIGIT represent such a family of pairwise receptors in humans and mice and interact with the ligands CD155 and CD112 (Bottino et al., 2003, Fuchs et al., 2004, Tahara-Hanaoka et al., 2004, Seth et al., 2007, Stanietsky et al., 2009, Stanietsky et al., 2013). Remarkably, LAQ differentially regulated the expression of these receptors by increasing the frequencies of DNAM-1+ and TACTILE+ NK cells and by simultaneously decreasing the frequency of TIGIT+ NK cells.

The ligand CD155, a member of the nectin-like protein family, is expressed on a variety of cells including endothelial cells, diverse tumor cells (Bottino et al., 2003, Tahara-Hanaoka et al., 2004, Lakshmikanth et al., 2009) as well as different immune cells such as T cells, NK cells, NKT cells, B cells, monocytes and dendritic cells (Maier et al., 2007).

Binding of the activating receptor DNAM-1 to its ligand increases the cytotoxic activity of NK cells towards the respective target cell (Bottino et al., 2003, Tahara-Hanaoka et al., 2004). DNAM-1 plays an important role in NK cell-mediated tumor surveillance (Lakshmikanth et al., 2009) and the regulation of immune cells (Pende et al., 2006, Ardolino et al., 2011). A single nucleotide polymorphism in the gene of DNAM-1, leading to the amino acid substitution glycine to serine at position 307 , is associated with an increased risk to develop multiple autoimmune diseases, including diabetes type I, rheumatoid arthritis and MS (Hafler et al., 2009, Wieczorek et al., 2009, Qiu et al., 2013). The mutation at position 307 is located within the intracellular domain of the protein, which is important for DNAM-1 signaling. The functional impact of this mutation has not yet been characterized and it is thus unknown if and - if so - how it alters DNAM-1 signaling. Still, these studies point towards a major impact of DNAM-1 on the pathogenesis of autoimmunity (de Andrade et al., 2013). In vitro studies indicate a regulatory role of DNAM-1+ NK cells, by limiting T cell activation. CD155 expression was upregulated on activated T cells upon stimulation by superantigen and on dendritic cells during the maturation, which rendered both cell types susceptible to lysis by DNAM-1+ NK cells (Pende et al., 2006, Ardolino et al., 2011). Both, direct lysis of activated T cells and killing of antigen-presenting cells could contribute to NK cellmediated inhibition of $\mathrm{T}$ cell activation.

TACTILE, the second activating receptor of this pairwise receptor family was first cloned in 1992 and is expressed on T cells and NK cells (Wang et al., 1992). Despite this early discovery, the function of TACTILE is less well characterized. TACTILE binds to CD155, which confers stable adhesion of NK cells to the target cells and enhances their cytotoxic effector functions (Fuchs et al., 2004, Seth et al., 2007). 
TIGIT is the inhibitory receptor belonging to this pairwise receptor family. Antagonistic to DNAM-1 and TACTILE, binding of TIGIT to CD155 inhibits NK cell cytotoxicity and reduces IFNY secretion (Stanietsky et al., 2009, Stanietsky et al., 2013). Recently, a mechanism for this inhibitory effect has been proposed by Liu and colleagues, who reported a disruption of granule polarization within the NK cell upon TIGIT engagement, which led to subsequent reduction of cytotoxic activity (Liu et al., 2013).

Different studies provide evidence that DNAM-1, TACTILE and TIGIT compete for the binding of CD155 (Stanietsky et al., 2009, Yu et al., 2009, Levin et al., 2011, Stanietsky et al., 2013). Interestingly the inhibitory receptor TIGIT displays the highest affinity for CD155 (Yu et al., 2009, Stanietsky et al., 2013). Functionally, this translates into a dominant inhibition of TIGIT over the activating effect of DNAM-1 (Stanietsky et al., 2009, Stanietsky et al., 2013). The data provided in this thesis indicate that LAQ considerably shifts the balance within this pairwise receptor family towards higher NK cell activity, by increasing the activating signals (DNAM-1 and TACTILE) and decreasing the corresponding inhibitory signal (TIGIT).

Additionally, LAQ treatment slightly but significantly enhanced the frequency of NKG2D+ NK cells. NKG2D is an activating receptor expressed on NK cells and T cells (Bauer et al., 1999), which binds to members of the retinoic acid early-inducible protein family (RAE) and H-60 in mice (Diefenbach et al., 2000, Lodoen et al., 2003) and to MHC class I polypeptide-related sequence A and B (MICA and MICB) and UL-16 binding proteins (ULBPs) in humans (Bauer et al., 1999, Cosman et al., 2001, Raulet, 2003). The interaction of NKG2D with its ligands promotes NK cellmediated cytokine secretion and cytotoxicity (Diefenbach et al., 2000, Lodoen et al., 2003). The role of NKG2D in the pathogenesis of MS and EAE is still unclear. In vitro experiments revealed NKG2D-dependent killing of oligodendrocytes, suggesting that NKG2D+ NK cells could be harmful within the CNS (Saikali et al., 2007). On the other hand, antigen-dependent stimulation of T cell proliferation led to an increased expression of NKG2D ligands on activated T cells and rendered them susceptible to NK cell-mediated lysis, favoring a beneficial role of NKG2D+ NK cells in the periphery (Rabinovich et al., 2003, Cerboni et al., 2007). Interestingly, a human study provided evidence for increased serum titers of the NKG2D ligand MICB in MS patients. MICB was especially elevated during the relapse phase, indicating that high serum levels of soluble MICB might be associated with disease activity (Fernandez-Morera et al., 2008). In tumor patients, stimulation of NKG2D+ NK cells with soluble ligands induces the internalization and degradation of NKG2D, which in turn impairs the responsiveness of these effector cells towards tumor cells and thus reduces tumor cell clearance (Groh et al., 2002). These findings suggest an inhibitory role of soluble NKG2D ligands on the activity of NK cells, which could potentially contribute to relapse 
rates in MS patients. In this setting, the LAQ-dependent increase of NKG2D+ NK cells could possibly compensate for a downregulation of NKG2D, which might be mediated by the presence of soluble MICB in MS patients.

One important feature of the regulation of NK cell activity is the discrimination between "self" and "non-self". For this purpose, NK cells express a variety of receptors that interact with MHC class I molecules. In mice, these receptors belong to the family of L49 receptors, which comprises inhibitory as well as activating receptors (Ortaldo et al., 2000, Natarajan et al., 2002). In the present study, the effect of LAQ on NK cells expressing the receptors Ly49C, I, F and H were investigated by using the monoclonal antibody 14B11, which detects all these receptors simultaneously (Corral et al., 1999). Ly49C, I and F are inhibitory receptors (Coles et al., 2000, Benoit et al., 2005, Orr et al., 2010), whereas Ly49H is an activating receptor which recognizes the glycoprotein m157 of the mouse cytomegalovirus (Corral et al., 1999, Ortaldo et al., 2000, Orr et al., 2009). LAQ treatment decreased the frequency of $L y 49 \mathrm{C} / \mathrm{I} / \mathrm{F} / \mathrm{H}+\mathrm{NK}$ cells. Since the antibody mainly detects inhibitory receptors this effect was interpreted as treatment-related reduction of inhibitory NK cell receptors. Beside the receptors from the Ly49 family, LAQ also diminished the frequency of the inhibitory receptor TIGIT, as discussed above. EAE severity in C57BI/6 mice is reduced by a decrease of inhibitory NK cell signals, as shown after blocking of the interaction between the inhibitory receptor NKG2A and its ligand and the subsequently enhanced NK cell activity (Lu et al., 2007, Leavenworth et al., 2010).

Next, this work investigated whether receptors which are shared by other immune cells are specifically regulated on NK cells. Therefore, effects of LAQ on the expression of DNAM-1, TACTILE and TIGIT were assessed on T cells. Interestingly, the pattern of LAQ-mediated regulation differed between NK cells and T cells. The investigation of effects of LAQ on DNAM-1, TACTILE and TIGIT on T cells, revealed no alteration of TACTILE+ and TIGIT+ T cells, but a decreased frequency of DNAM-1+ T cells upon LAQ treatment.

Regarding T cells, the activating receptor DNAM-1 is associated with proliferation and differentiation (Shibuya et al., 2003). DNAM-1 is upregulated during Th1 differentiation and downregulated during Th2 differentiation (Dardalhon et al., 2005) and is highly expressed on Th1 and Th17 cells (Lozano et al., 2013). Antibody-mediated blocking of DNAM-1 inhibits T cell activation and proliferation and reduces the production of the pro-inflammatory cytokines IL-17 and IFNY (Lozano et al., 2013). Reduced frequencies of DNAM-1+ T cells after LAQ treatment are thus in line with the observed inhibition of pro-inflammatory $T$ cell subsets upon treatment which were discussed before (see 4.1). 
In contrast to DNAM-1, the inhibitory receptor TIGIT is a negative regulator of $\mathrm{T}$ cell activation. Binding of TIGIT to its ligands reduces Th1 and Th17 differentiation (Lozano et al., 2012, Lozano et al., 2013) and TIGIT is associated with an anti-inflammatory T cell phenotype (Joller et al., 2011, Lozano et al., 2012). LAQ did not decrease the frequency of TIGIT+ T cells in contrast to its action on NK cells.

Taken together, these data indicate that LAQ treatment selectively increases the activation of NK cells, which potentially contributes to its mechanism of action in MS and EAE.

\subsubsection{LAQ enhanced NK cell effector functions}

Next, this work evaluated the functional impact of the LAQ-induced upregulation of activation markers on NK cells. Therefore, NK cell effector functions were investigated in vitro by sorting out NK cells from LAQ- or vehicle-treated animals. In line with previous studies on human NK cells (Fu et al., 2011), CD27+ NK cells killed less efficiently B16F10 melanoma cells than CD11b+ NK cells. In vivo LAQ treatment resulted in higher killing efficiencies of CD27+ as well as CD11b+ NK cells. Furthermore, IFNy production upon ex vivo stimulation was measured in NK cells derived from LAQ- and vehicle-treated animals. NK cells, pre-treated with LAQ in vivo, were characterized by an elevated frequency of IFN $\gamma+$ cells and higher IFN $\gamma$ concentrations in the supernatant. In summary, LAQ markedly affected the main effector functions of NK cells evidenced by increased NK cell cytotoxicity and IFNY production.

The increased killing efficiency of CD27+ as well as CD11b+ NK cells upon LAQ treatment points towards a general LAQ effect on NK cells irrespective of the subpopulation. This is in line with the LAQ-mediated upregulation of the activation marker CD69 on the surface of both, CD27+ and CD11b+ NK cells, reported in this work. The enhancement of NK cell-mediated killing of B16F10 melanoma cells could be explained by the increased expression of DNAM-1 on LAQtreated NK cells (compare 3.3.2). The DNAM-1 ligand CD155 is expressed on B16F10 melanoma cells (Lakshmikanth et al., 2009) and DNAM-1 is thought to be one crucial NK cell surface receptor for the recognition of these cells. Genetic depletion of DNAM-1 renders NK cells irresponsive to the challenge with B16F10 cells in vivo and in vitro (Gilfillan et al., 2008).

The promoting effect of LAQ on NK cell effector functions is substantiated by comparable observations published for its precursor substance roquinimex. In vivo treatment with roquinimex increases NK cell mediated lysis of tumor cells in vitro in mice (Kalland et al., 1985, Karussis et al., 1993) and humans (Bengtsson et al., 1992). Further, roquinimex boosts the rejection of B16F10 cells in mice in vivo and blocks the development of metastases (Kalland, 1986). Due to the different methodologies used to isolate NK cells in the 1980s and early 1990s in contrast to the 
present study, comparisons have to be drawn with caution. Still, the data provided on effects of roquinimex on NK cell cytotoxicity resemble those observed for $L A Q$ in the present work. LAQ also increased the cytokine secretion of NK cells in addition to the higher cytotoxicity. None of the studies on roquinimex assessed its effects on the cytokine production by NK cells.

In summary, LAQ treatment markedly shifted NK cell subsets towards more CD27+ NK cells, led to the upregulation of activating NK cell receptors and increased NK cell effector functions in mice.

\subsubsection{Human NK cells in MS - a potential target for LAQ treatment?}

Human NK cells are classically divided into two distinct subpopulation according to their expression of the surface markers CD56 and CD16: CD56 bright CD16- regulatory NK cells and CD56 ${ }^{\text {dim }}$ CD16+ cytotoxic NK cells (Lanier et al., 1986). Alterations in these NK cell subpopulations could have an impact on the pathogenesis of MS, since a recently published report evidenced significantly increased frequencies of $\mathrm{CD} 56^{\mathrm{dim}}$ perforin+ NK cells among peripheral blood mononuclear cells (PBMCs) of patients with progressive MS compared to healthy controls (Plantone et al., 2013). Furthermore, the concept that increased numbers of CD56 bright $N K$ cells could be beneficial in MS is supported by studies which reveal a differential upregulation of the CD56 $6^{\text {bright }}$ subset in peripheral blood from MS patients in response to treatment with IFN及 (Saraste et al., 2007, Vandenbark et al., 2009, Martinez-Rodriguez et al., 2010) and daclizumab (Bielekova et al., 2006). For daclizumab the elevation of CD56 bright $N K$ cells was directly correlated with a positive treatment response (Bielekova et al., 2006). CD56 is not expressed on murine NK cells, but recently CD27 and CD11b were reported to be mainly expressed by human CD56 bright and CD56 ${ }^{\mathrm{dim}}$ NK cells, respectively (Fu et al., 2011), facilitating the comparison of human and murine data. Therefore, the LAQ-mediated increase of CD27+ or CD56 bright NK cells could be potentially beneficial for the treatment of MS, as previously described for other MS therapies.

Different activating and inhibitory receptors appear to be implicated in the pathogenesis of MS. The study of Martinez-Rodriguez and colleagues revealed an increased expression of the inhibitory receptors LILRB1 and NKG2A on NK cells from patients with progressive MS compared to healthy controls. Treatment with IFN $\beta$ also decreased the expression of LILRB1 on NK cells efficiently (Martinez-Rodriguez et al., 2010, Martinez-Rodriguez et al., 2011). Moreover, a recent report describes a negative association between the activating NK cell receptor gene KIR2DS1 and MS (Bettencourt et al., 2014). Considering the LAQ-mediated shift in the receptor profile of murine NK cells, LAQ therapy could also be beneficial for MS patients with increased expression of inhibitory NK cell receptors. 
Furthermore, MS is thought to be associated with defects in NK cell effector functions. Several studies show reduced in vitro killing efficiency of NK cells isolated from the peripheral blood of MS patients compared to healthy controls (Benczur et al., 1980, Hauser et al., 1981, Neighbour et al., 1982, Kastrukoff et al., 1998). The development of relapses and new MRI lesions seems to be associated with preceding phases of low NK cell activity (Kastrukoff et al., 2003). A few studies also reported no alteration in NK cell function of MS patients (Vervliet et al., 1983, Satoh et al., 1990). These diverging observations could be due to differences in the study populations, as NK cell activity is heterogeneous within MS patients as well as healthy controls (Kastrukoff et al., 2003). The study design could also profoundly influence the outcome, since an intra-individual oscillation of NK cell activity was observed in longitudinal studies (Kastrukoff et al., 1998, Kastrukoff et al., 2003). Given a defective phenotype of NK cells in MS with reduced effector functions, LAQ could potentially compensate for this defect by activating NK cells and by restoring their effector functions.

\subsection{LAQ-treated NK cells inhibited antigen-specific T cell proliferation in a contact-dependent manner}

The analysis of the NK cell phenotype and effector functions in LAQ-treated animals revealed a considerable activation of NK cells upon treatment, as discussed before (compare 4.2). In vivo, NK cells are part of a functional network and can regulate the process of immune activation by interacting with dendritic cells and T cells. Triple co-culture experiments of NK cells with dendritic cells and T cells were used to evaluate the effect of NK cells, pre-treated with LAQ or vehicle in vivo, on $\mathrm{MOG}_{35-55}$-induced T cell proliferation. The addition of LAQ-treated NK cells markedly reduced antigen-dependent T cell proliferation, evidenced by an increased frequency of non-proliferating T cells. Vehicle-treated NK cells also inhibited T cell proliferation to a certain extent, but less efficiently than LAQ-treated NK cells. Further, the NK cell-mediated reduction of $\mathrm{MOG}_{35-55}$-stimulated T cell proliferation was dependent on direct cell contact, as separation of NK cells from T cells and dendritic cells in a transwell plate completely abolished the inhibitory effect. Taken together, LAQ-treated NK cells inhibited antigen-dependent $T$ cell proliferation more efficiently than control NK cells and this inhibition was mediated by direct cell contact.

The observed inhibitory effect of NK cells on antigen-dependent T cell proliferation is in line with previously published studies. Murine, rat and human NK cells have been shown to reduce antigen-triggered T cell proliferation in a cell contact-dependent manner in vitro (Zhang et al., 1997, Smeltz et al., 1999, Wolf and Swanborg, 2001, Takao et al., 2010). 
In the applied triple co-culture system, NK cells could reduce T cell proliferation either directly by interacting with the activated $T$ cells or indirectly by interacting with the antigenpresenting dendritic cells. Regarding the mechanism, direct killing of activated T cells or dendritic cells could be one way how NK cells reduce T cell proliferation. The clear dependency on direct cell contact could argue for this hypothesis, as NK cells mediate their cytotoxicity towards target cells via contact dependent pathways involving granule exocytosis (perforin, granzyme), Fas-Fasligand engagement and TRAIL-TRAIL-ligand interaction (Segal, 2007). Further, several cell culture experiments demonstrate that NK cells efficiently induce cell death of immature dendritic cells (Carbone et al., 1999, Wilson et al., 1999, Ferlazzo et al., 2001, Ferlazzo et al., 2002, Piccioli et al., 2002, Della Chiesa et al., 2003, Ferlazzo et al., 2003, Hayakawa et al., 2004, Pende et al., 2006, Alter et al., 2010) and activated T cells (Smeltz et al., 1999, Rabinovich et al., 2003, Xu et al., 2005, Bielekova et al., 2006, Takao et al., 2010, Ardolino et al., 2011, Nielsen et al., 2012) by direct interaction of activating NK cell receptors with their cognate ligands on the target cells.

Therefore, this thesis investigated whether the LAQ-specific reduction in $\mathrm{T}$ cell proliferation was due to NK cell-mediated killing of activated T cells and/or dendritic cells in the triple co-culture. The presence of apoptotic $\mathrm{T}$ cells and dendritic cells was evaluated by Annexin $\mathrm{V}$ and 7-AAD staining. Indeed, the addition of NK cells to the co-culture led to a considerable increase of late apoptotic/dead T cells and dendritic cells. However, the degree of NK cellmediated cell death did not differ between NK cells derived from LAQ- and vehicle-treated animals.

Considering these data, the LAQ-specific inhibition of T cell proliferation can probably not be attributed to increased killing of T cells and dendritic cells. Still, these observations do not completely exclude a contribution of NK cell-mediated killing to the LAQ-specific reduction of T cell proliferation. Significant differences in the killing efficiency of tumor cells were only observed at an effector/target ratio of 5:1 (compare 3.3.3.1) between LAQ-treated and control NK cells. The effector/target ratios used in the triple co-culture system were 1:1 for NK cells versus dendritic cells and 0.5:1 for NK cells versus T cells. Possibly, these effector/target ratios were too low to detect marked differences between LAQ- and vehicle-treated NK cells.

To further dissect the effects of LAQ-treated NK cells on activated T cells and bone marrow-derived dendritic cells, NK cells were co-cultured with each cell type separately.

Simple co-culture experiments of NK cells with bone marrow-derived dendritic cells were performed to evaluate NK cell-mediated killing of dendritic cells in the absence of activated T cells. These experiments did not reveal any NK cell-mediated lysis of bone marrow-derived dendritic cells in culture, irrespective of LAQ treatment. These findings strengthen the hypothesis 
that killing of dendritic cells is not of central importance for LAQ-treated NK cells to reduce antigen-dependent T cell proliferation.

However, these results appear to be in contrast with several published studies describing efficient NK cell-mediated lysis of dendritic cells in vitro. One reason for the conflictive results could be the pre-treatment of NK cells with IL-2, applied in most published studies (Chambers et al., 1996, Carbone et al., 1999, Wilson et al., 1999, Ferlazzo et al., 2001, Spaggiari et al., 2001, Ferlazzo et al., 2002, Della Chiesa et al., 2003, Pende et al., 2006, Alter et al., 2010). In vitroactivated NK cells might have been more cytotoxic than ex vivo-derived NK cells in this thesis. Further, differences in the NK cell to dendritic cell ratios could contribute to the discrepancy, since this parameter seems to have a marked impact on the killing efficiency of NK cells (Piccioli et al., 2002).

Additionally, the lack of NK cell-mediated lysis of dendritic cells in the simple co-culture is contrary to the NK cell-induced apoptosis of dendritic cells reported in the triple co-culture in the present work. One possible explanation for these conflicting results could lie within the sensitivities of the different methods used to analyze cell death, as the crystal violet assay is certainly less sensitive than the Annexin V and 7-AAD staining. Further, NK cells are potentially more activated and therefore more cytotoxic in the triple co-culture system, where the interaction of CD40 on dendritic cells with CD40L on T cells potentially induces the secretion of the cytokine IL-12 by dendritic cells (Ma and Clark, 2009). IL-12 enhances NK cell activation and cytotoxicity (Nielsen et al., 2012) and could increase the NK cell-mediated killing of dendritic cells in triple co-cultures compared to single co-cultures.

In an additional set of experiments, LAQ- and vehicle-treated NK cells were co-cultured with CD4+ T cells and proliferation was induced in an antigen-independent manner by agonistic anti-CD3 and anti-CD28 antibodies. This experimental setup did not reveal any inhibitory effect of NK cells on antigen-independent T cell proliferation. Neither LAQ-treated, nor control NK cells reduced T cell proliferation in this setting.

The minor effect of NK cells on T cell proliferation upon antigen-independent stimulation potentially indicates that T cells are not the direct target of NK cells in the triple co-culture and that the presence of dendritic cells is mandatory for the NK cell-mediated inhibition of T cell proliferation. However, a direct interaction of NK cells and activated T cells cannot be ruled out, since the strong stimulus of anti-CD3 and anti-CD28 antibodies in the applied concentrations possibly overrides the regulatory effect of NK cells.

Assuming an indirect effect on T cell proliferation via the interaction of NK cells with dendritic cells, different mechanisms can be discussed. Nakayama and colleagues have proposed that the presentation of dendritic cell MHC class II molecules on the surface of NK cells could 
account for the inhibition of T cell proliferation. This mechanism is based on direct cell contact between NK cells and dendritic cells, which enables the transfer of MHC class II molecules from dendritic cells to NK cells by trogocytosis. MHCII-dressed NK cells subsequently compete with dendritic cells for the binding of antigen-specific T cells. Since NK cells lack the co-stimulatory molecules CD80 and CD86, the interaction of T cells with MHCII-dressed NK cells does not induce $\mathrm{T}$ cell proliferation and the competition with the potent antigen-presenting dendritic cells leads to a reduction of T cell proliferation (Nakayama et al., 2011).

Contact-dependent cytokine secretion is another possible mechanism by which NK cells could indirectly control T cell proliferation. It has been shown that the engagement of different activating receptor combinations on human NK cells in vitro potently induced the secretion of specific pro-inflammatory cytokines (Fauriat et al., 2010). Beside pro-inflammatory cytokines, NK cells also secrete anti-inflammatory cytokines like IL-10 and NK cell-derived IL-10 has been shown to reduce antigen-dependent T cell proliferation in vitro (Deniz et al., 2008). Thus, possibly the specific engagement of a panel of receptors on LAQ-treated NK cells via direct cell contact with dendritic cells could trigger an anti-proliferative cytokine secretion by these NK cells.

In brief, LAQ-treated NK cells specifically reduced antigen-dependent T cell proliferation in the presence of dendritic cells, but did not alter antigen-independent T cell proliferation in the absence of dendritic cells. Furthermore, NK cell-mediated apoptosis was not enhanced by LAQ treatment. On the basis of these results and previously published data the following hypothesis could be made: NK cells may directly interact with dendritic cells in the triple co-culture, which may impair dendritic cell-mediated T cell activation, potentially by reducing the expression of costimulatory molecules and MHC class II or by altering the cytokine profile of dendritic cells. Alternatively, the engagement of specific NK cell receptors by molecules on the surface of dendritic cells may trigger the contact-mediated secretion of anti-inflammatory cytokines from NK cells. Both mechanisms could subsequently contribute to reduced T cell proliferation. The specificity of LAQ-treated NK cells for reducing the antigen-dependent T cell proliferation may arise from the differentially regulated receptor profile upon LAQ treatment (compare 3.3.2.2). 


\subsection{LAQ exerted independent effects on NK cells and dendritic cells}

\subsubsection{LAQ-mediated changes displayed a similar kinetic in NK cells and dendritic cells and were delayed in T cells}

This study provided evidence for in vivo effects of LAQ on T cells, dendritic cells and NK cells. It was further investigated whether these effects represent parallel or sequential mechanisms of action. First, time course experiments were performed to elucidate the kinetics of LAQ-induced changes. NK cells responded promptly to LAQ treatment, indicated by the upregulation of TACTILE, CD69 and NKG2D after one or two days of LAQ therapy, respectively. Dendritic cells displayed similar kinetics, with increased frequencies of CD80+ and CD86+ dendritic cells after two or three days of LAQ treatment. Total dendritic cells were considerably decreased after three days, which corresponds to the half life of dendritic cells in the murine spleen (Kamath et al., 2000). In contrast, the onset of treatment effects was delayed in T cells. Analysis of naïve T cells, effector memory T cells, Th1 and Th17 cells revealed no drug-related alterations after four days of LAQ therapy.

These results are in line with previous reports. Decreased differentiation of transferred 2D2 T cells into Th1 and Th17 cells was observed after six days of LAQ therapy (Jolivel et al., 2013) and LAQ reduced VLA-4-dependent T cell adhesion after seven days of treatment (Wegner et al., 2010).

The temporal analysis provided in this work, revealed fast kinetics of LAQ-induced changes in NK cells and dendritic cells, preceding those observed for T cells, which points towards a sequential mechanism of action. In this concept, LAQ would exert early and direct effects on innate immune cells, which in turn modulate subsequent T cell responses.

Data presented in this work, support this hypothesis. First, LAQ-induced changes in NK cell subpopulations and CD69 regulation were independent of T and B cells, since LAQ exerted comparable effects in wild type and Rag1-deficient animals. Further, NK cells, pre-treated with $\mathrm{LAQ}$ in vivo, shaped the $\mathrm{T}$ cell response to antigenic stimulation in vitro by inhibiting $\mathrm{T}$ cell proliferation (see 4.3). Finally, in vitro LAQ treatment failed to directly inhibit pro-inflammatory cytokine production in T cells, as discussed before (see 4.1).

The effect of LAQ on antigen-presenting cells has been extensively studied by other groups and the results further underline the before mentioned hypothesis. The study of Jolivel and colleagues also revealed very fast kinetics of LAQ-mediated alterations in dendritic cells, with the first changes in CD86 expression after one single dose of LAQ. Generally, LAQ treatment decreased the frequency of antigen-presenting cells and induced a more regulatory phenotype 
(Schulze-Topphoff et al., 2012, Thöne et al., 2012, Jolivel et al., 2013). These changes potentially interfere with T cell priming and differentiation as discussed before (see 4.1).

Taken together, these data support the concept of an early and direct effect of LAQ on innate immune cells with a subsequent indirect modulation of the priming and the differentiation of T cells.

\subsubsection{The effects of LAQ on dendritic cells were independent of NK cells in vivo}

Time course experiments revealed similar kinetics for LAQ-induced changes in NK cells and dendritic cells. To further dissect an interrelation of drug effects on these two cell types, NK cells were depleted prior to treatment and effects of LAQ on dendritic cells were evaluated. The reduction of total splenic dendritic cells and myeloid dendritic cells was comparable in NK cellcompetent and NK cell-depleted mice treated with LAQ. Thus, NK cells seem to be dispensable for the action of LAQ on dendritic cells.

These results are in line with previously published data, reporting a direct effect of LAQ on dendritic cells in vitro. LAQ-treatment during the generation of human monocyte-derived and murine bone marrow-derived dendritic cells in vitro decreases the ability of these cells to produce pro-inflammatory cytokines and chemokines upon stimulation. Further, human monocyte-derived dendritic cells, pre-treated with LAQ in vitro, are less efficient in stimulating the proliferation of allogeneic T cells in vitro (Jolivel et al., 2013).

Consequently, LAQ seems to act directly on dendritic cells and NK cells do not interfere with this regulation in vivo.

\subsubsection{LAQ treatment directly induced NK cell activation}

The effects of LAQ on dendritic cells were independent of NK cells in vivo, as discussed before. Similarly, NK cells were found to be a direct target of LAQ in vitro. Culture of naïve NK cells with LAQ revealed a direct in vitro activation of NK cells by the compound in the absence of any other cell type. Co-culture of NK cells with bone marrow-derived dendritic cells, pre-treated with $L A Q$, could not mimic this effect.

The idea of NK cells as direct target of LAQ treatment is supported by a published study investigating its precursor substance roquinimex. In this work, human adherent lymphokine activated killer cells displayed increased cytotoxicity against NK cell-sensitive K562 tumor cells upon in vitro treatment with roquinimex (Vaz et al., 1995).

In general, LAQ seems to directly target different innate immune cells and CNS-resident cells to exert its full immunomodulatory mechanism of action. Concerning innate immunity, in 
vitro effects of LAQ have been previously described for monocytes (Mishra et al., 2012) and dendritic cells (Jolivel et al., 2013). Regarding CNS-resident cells, LAQ interfered with astrocytes (Brück et al., 2012) and microglia (Mishra et al., 2013) in vitro.

In consequence, the presented in vitro data identify NK cells as a novel direct target of LAQ.

\subsubsection{LAQ interferes with different signaling pathways depending on the target cell}

LAQ treatment in vivo and in vitro inhibits different pro-inflammatory signaling pathways depending on the target cell investigated. Most evidence is available for effects of LAQ on the NF-KB pathway. Inhibitory effects on this signaling pathway have been directly shown in human monocytes (Mishra et al., 2012), human monocyte-derived dendritic cells, murine bone marrowderived dendritic cells (Jolivel et al., 2013) and murine and human astrocytes (Brück et al., 2012). In human microglia LAQ reduced the activation of AKT (protein kinase B), an activator of the NF-KB pathway (Dan et al., 2008) and thereby indirectly reduced NF-KB signaling (Mishra et al., 2013). In murine monocytes, LAQ inhibits the Janus kinase (JAK) and Signal Transducer and Activator of Transcription (STAT) pathway, indicated by reduced phosphorylation of STAT1 (Schulze-Topphoff et al., 2012). It is controversial whether mitogen-activated protein (MAP) kinase activities are also altered by LAQ treatment (Mishra et al., 2012, Schulze-Topphoff et al., 2012).

The described interference of LAQ with NF-KB and JAK-STAT signaling leads to a reduction of pro-inflammatory responses in cells, which is potentially beneficial for the treatment of MS and EAE. In contrast to monocytes and dendritic cells, LAQ rather activated than attenuated NK cells, as reported in this work.

NF-KB and JAK-STAT signaling play important roles in the activation of NK cell functions. Hyperactivation of NF-KB signaling blocks the maturation of NK cells and increases the expression of CD69 (Samson et al., 2004). Further, reduced NF-KB activity in NK cells results in a decreased expression of perforin (Zhou et al., 2002) and impaired cytotoxicity towards tumor cells in vitro (Orange et al., 2002).

STAT1 becomes phosphorylated and thereby activated upon cytokine signaling (Rawlings et al., 2004). The stimulation of NK cells by type I interferons activates STAT1 and leads to increased cytotoxicity (Nguyen et al., 2002). STAT1-deficient NK cells are characterized by impaired cytotoxicity towards tumor cells in vitro and reduced IFNY secretion upon stimulation (Lee et al., 2000, Fortin et al., 2013). In contrast, one recent report described an opposite function of STAT1 phosphorylation on NK cell activation. A point mutation at serine727, which blocked

cyclin-dependent kinase 8-mediated phosphorylation of STAT1, induced activation and cytotoxicity of NK cells (Putz et al., 2013). 
In conclusion, further studies are required to elucidate the exact mechanism by which LAQ increases the activation of NK cells.

\subsection{NK cell depletion decreased the therapeutic efficiency of LAQ in EAE}

In this study, NK cells were identified as a promising target for LAQ. The significance of NK cells within the mechanism of LAQ action remained to be investigated. For this purpose, the therapeutic efficiency of LAQ in EAE was evaluated in NK cell-competent and NK cell-depleted Th/+ mice. The effects of LAQ were clearly dependent on the presence of NK cells. LAQ treatment markedly reduced EAE symptoms in NK cell-competent animals, whereas NK celldepleted animals showed a markedly weaker response to LAQ therapy and displayed only a transient drug-related amelioration of disease. In the vehicle-treated group, NK cell depletion did not alter EAE severity per se. In terms of mortality, LAQ treatment conferred total protection from death to NK cell-competent animals, which was partly abrogated by NK cell depletion. Survival rates of approximately $50 \%$ were observed in the control groups irrespective of NK cell depletion. Thus, NK cells seem to play a major role for the protective effect of LAQ in EAE.

LAQ markedly reduced EAE severity in NK cell-competent Th/+ mice. Still, the therapeutic effect in Th/+ mice was lower compared to wild type mice, where preventive treatment at a dose of $25 \mathrm{mg} / \mathrm{kg}$ was fully protective in this study. This discrepancy is likely to be related to the fact that Th/+ mice develop an accelerated and exacerbated EAE course compared to wild type animals upon challenge with encephalitogenic antigens (Litzenburger et al., 1998). The described aggravation of disease was also paralleled by the relatively high mortality rate (approximately $50 \%$ ) observed for vehicle-treated Th/+ mice in this work. Parallel assessment of the effects of LAQ on the survival rate of wild type animals could not be determined in this study, since death of wild type mice was an exceptional event in these experiments. However, the beneficial effect of LAQ on the survival of Th/+ mice is in line with previously published data, which demonstrate an improved survival upon LAQ treatment in wild type mice (Aharoni et al., 2012).

Th/+ mice were chosen to investigate the impact of NK cells on the efficiency of LAQ in EAE for two distinct reasons: First, the incidence of EAE upon immunization is very high in this animal model (Litzenburger et al., 1998). This aspect was important, since the animals in this experimental setting were exposed to high stress levels due to frequent intraperitoneal treatment with either antibody or PTX in addition to the daily oral gavage of LAQ or vehicle. Second, naïve Th/+ mice display high serum titers of pathogenic antibodies (Litzenburger et al., 1998), which could bind to CNS intrinsic structures upon opening of the blood-brain barrier. These high levels of pathogenic antibodies might be crucial since antibody-dependent cell- 
mediated cytotoxicity (ADCC) is one important effector function of NK cells (Seidel et al., 2013). The physiological role of ADCC is to recognize and kill antibody-coated infected cells, which contributes to the clearance of infection. In the case of antibody-mediated autoimmunity, NK cells could potentially cause CNS injury by killing antibody-coated CNS cells. Therefore, it was of particular importance to test for protective or potentially detrimental effects of LAQ-mediated NK cell activation in the presence of pathogenic antibodies.

There is a controversial discussion on the role of NK cells in the pathogenesis of EAE. NK cell depletion with anti-NK1.1 antibody before immunization has been shown to exacerbate EAE in C57BI/6 mice (Zhang et al., 1997, Huang et al., 2006) and SJL/J mice (Xu et al., 2005), which suggests a regulatory role of NK cells. Furthermore, deletion of the chemokine receptor CX3CR1 resulted in a selective block of NK cell migration into the inflamed CNS and was associated with increased EAE-mediated mortality (Huang et al., 2006). A regulatory role of NK cells is additionally supported by the observation of BDNF-producing NK cells within the CNS of EAE mice (Hammarberg et al., 2000). In contrast, other studies provide evidence for an amelioration of EAE symptoms after depleting NK cells with anti-NK1.1 antibody prior to immunization, favoring a pro-inflammatory role of NK cells (Shi et al., 2000, Winkler-Pickett et al., 2008). It is difficult to explain these controversial findings, since all the cited studies used an anti-NK1.1 antibody to deplete NK cells prior to immunization and effective depletion was controlled before EAE induction. Potentially, the different results could be partly explained by the fact that all experiments were carried out at different institutions and animal facilities. Each animal facility displays its typical pattern of commensal microbiota, which has been demonstrated to influence the development of EAE (Ochoa-Reparaz et al., 2009, Ochoa-Reparaz et al., 2010, Ochoa-Reparaz et al., 2010, Berer et al., 2011). Further, commensal microbiota are necessary for the efficient priming of NK cells by dendritic cells and monocytes. Different patterns of bacteria within the gut flora exert immunomodulatory properties by altering the crosstalk between dendritic cells and NK cells, which modulates NK cell priming (Rizzello et al., 2011, Ganal et al., 2012). These factors are likely to contribute to the different outcomes observed after NK cell depletion in EAE.

In conclusion, NK cell depletion profoundly reduced the therapeutic efficiency of LAQ in Th/+ mice with EAE, arguing for a major contribution of NK cells within the mechanism of action of LAQ. 


\subsection{Outlook}

The present work has identified NK cells as a new target of LAQ therapy and provided evidence for a central role of the drug-related NK cell activation within the protective mechanism of action of LAQ in EAE. Further research is required to fully understand the effect of LAQ on NK cells and the functional consequences in MS and EAE.

First, LAQ differentially regulated the expression of the NK cell receptors DNAM-1, TACTILE and TIGIT, all belonging to the same pairwise receptor family. It would be intriguing to investigate the functional impact of this altered receptor profile in vivo and in vitro, using specific receptor-deficient mice or blocking antibodies.

Second, NK cell depletion markedly reduced the protective effect of LAQ in EAE. However, it is necessary to exclude a significant contribution of NKT cells in order to substantiate a central role of NK cells only for the therapeutic efficiency of LAQ in EAE. This could be achieved by assessing the effects of LAQ treatment on adoptive transfer EAE in Rag1 ${ }^{-1-}$ in comparison to Rag $1^{-1-}$ gamma $(\mathrm{c})^{-/-}$mice, the former lacking $B, T$ and NKT cells and the latter being deficient for $B, T, N K T$ and NK cells.

Third, LAQ has been shown to interfere with different pro-inflammatory signaling pathways in immune cells and CNS-resident cells. The effect of LAQ on signaling pathways within NK cells has not been assessed in the present study. Evaluating specific LAQ-induced alterations of signaling cascades in NK cells could be of central importance to obtain a deeper understanding why LAQ enhances the activity of NK cells, whereas it rather attenuates other immune cells.

Fourth, the present work mainly focused on potential consequences of LAQ treatment on the regulatory role of NK cells during the activation of T cell responses. However, NK cells can be found in the inflamed CNS of EAE animals and also exert beneficial effects by producing BDNF (Hammarberg et al., 2000). Therefore, it would be important to analyze effects of LAQ on the capacity of NK cells to migrate into the CNS and to produce BDNF in order to investigate potential neuroprotective properties of LAQ-treated NK cells.

Finally, it will be crucial to test whether LAQ treatment exerts similar effects on human NK cells. It is of central relevance to validate the experimental data in MS patients and healthy controls and to evaluate the significance of LAQ-mediated alterations of NK cells for the therapy of MS patients. 


\section{Summary and conclusions}

The new immunomodulatory substance $L A Q$ is currently under investigation for the treatment of relapsing remitting multiple sclerosis. Phase III clinical trials indicate beneficial effects of LAQ treatment on relapse rate, brain atrophy and disability progression in RRMS patients. In EAE studies, LAQ efficiently inhibited disease induction and development, which was attributed to its ability to reduce antigen presentation and the subsequent suppression of proinflammatory $T$ cell differentiation. The present study aimed to investigate effects of the immunomodulator LAQ on innate and adaptive immune cells in mice with EAE, with a special focus on NK cells.

First, this work aimed to analyze the effects of preventive LAQ treatment on EAE severity and different T cell subpopulations. Preventive treatment with $25 \mathrm{mg} / \mathrm{kg}$ LAQ completely inhibited EAE induction in C57BI/6J mice and shifted the balance from pro-inflammatory Th1 and Th17 cells to anti-inflammatory regulatory T cells in vivo. The lack of in vitro effects of LAQ treatment on proinflammatory cytokine secretion, together with the delayed onset of LAQ-mediated alterations in T cells, indicate an indirect action of LAQ on T cells, which is in line with recently published data. Altogether, the LAQ-mediated shift from pro- to anti-inflammatory T cell subpopulations appears to be one central aspect of its mechanism of action in EAE.

Moreover, the present study evaluated the effects of preventive LAQ treatment on antigen presenting cells, by investigating dendritic cells. LAQ therapy drastically reduced the frequencies of total splenic dendritic cells and myeloid dendritic cells and led to the upregulation of the co-stimulatory molecules CD80 and CD86 within the first days of treatment. Considering these results and previously published data, LAQ appears to reduce and to alter antigen presentation, which in combination results in a decreased pro-inflammatory $T$ cell response and thereby attenuates EAE severity.

The central aim of this study was to investigate the effects of preventive LAQ treatment on NK cells and to evaluate the impact of NK cells on the therapeutic efficiency of LAQ in EAE.

In vivo, the administration of LAQ induced a considerable shift in NK cell subpopulations towards more CD27+ and fewer CD11b+ NK cells, whereas total splenic NK cell frequencies remained unaltered. Additionally, LAQ treatment markedly increased the activation of NK cells. This was evidenced by the pronounced upregulation of the activation marker CD69 and the altered balance of activating and inhibitory receptors on the surface of NK cells in favor of more 
activating signals upon LAQ therapy. Notably, LAQ treatment specifically interfered with one pairwise receptor family consisting of DNAM-1, TACTILE and TIGIT, enhancing the activating signals and attenuating the corresponding inhibitory signal. Most interestingly, this regulation was specific for NK cells, since LAQ led to different regulatory effects on T cells with regard to DNAM-1, TACTILE and TIGIT, matching the more anti-inflammatory T cell phenotype in LAQtreated animals.

Functionally, in vivo LAQ treatment boosted NK cell effector functions. LAQ significantly enhanced the cytotoxicity of CD27+ and CD11b+ NK cells towards B16F10 melanoma cells in vitro and increased the frequency of IFN + NK cells as well as the secretion of IFNY by NK cells. Moreover, NK cells purified from LAQ-treated animals efficiently inhibited antigen-dependent T cell proliferation in vitro.

Additional experiments analyzed whether NK cells represent a novel direct target of LAQ. NK cells responded very quickly to LAQ treatment in vivo and the effects of LAQ on NK cells were independent of B and T cells. In vitro therapy experiments revealed a direct effect of LAQ on NK cell activation in the absence of any other cell type, whereas co-culture with dendritic cells, pretreated with $L A Q$, could not mimic this effect.

Having identified NK cells as a new direct target of LAQ treatment, it was crucial to investigate the impact of NK cells on the therapeutic efficiency of LAQ in EAE. Experiments using Th/+ mice revealed a central role for NK cells within the mechanism of action of LAQ in EAE. LAQ treatment markedly reduced EAE severity in NK cell-competent animals, whereas NK cell depletion considerably attenuated this beneficial effect, since NK cell-depleted animals displayed only a transient drug-related amelioration of disease. Regarding mortality, LAQ treatment conferred complete protection from death to NK cell-competent mice, which was markedly diminished upon NK cell depletion.

Taken together, the present work identified NK cells as a novel target for LAQ. Remarkably, the LAQ-mediated activation of NK cells appears to be crucial for the therapeutic efficiency of the substance in EAE. A large body of evidence indicates that MS is associated with reduced NK cell activity and that enhancing NK cell functions by different treatments is beneficial for MS patients. Considering these published data and the results provided in this work, LAQ treatment could potentially restore the functional defects of NK cells in MS patients and this could contribute to its protective mechanisms in MS. 


\section{References}

Aharoni R, Saada R, Eilam R, Hayardeny L, Sela M, Arnon R

Oral treatment with laquinimod augments regulatory T-cells and brain-derived neurotrophic factor expression and reduces injury in the CNS of mice with experimental autoimmune encephalomyelitis

J Neuroimmunol. 2012; 251(1-2):14-24

Aharoni R, Teitelbaum D, Arnon R

T suppressor hybridomas and interleukin-2-dependent lines induced by copolymer 1 or by spinal cord homogenate down-regulate experimental allergic encephalomyelitis

Eur J Immunol. 1993; 23(1):17-25

Aharoni R, Teitelbaum D, Sela M, Arnon R

Copolymer 1 induces $T$ cells of the T helper type 2 that crossreact with myelin basic protein and suppress experimental autoimmune encephalomyelitis

Proc Natl Acad Sci U S A. 1997; 94(20):10821-6

Ahlgren C, Oden A, Lycke J

High nationwide prevalence of multiple sclerosis in Sweden

Mult Scler. 2011; 17(8):901-8

Al-Falahi Y, Sand KL, Knudsen E, Damaj BB, Rolin J, Maghazachi AA

Splenic natural killer cell activity in two models of experimental neurodegenerative diseases

J Cell Mol Med. 2009; 13(8B):2693-703

Almohmeed YH, Avenell A, Aucott L, Vickers MA

Systematic review and meta-analysis of the sero-epidemiological association between Epstein Barr virus and multiple sclerosis

PLoS One. 2013; 8(4):e61110

Alter G, Kavanagh D, Rihn S, Luteijn R, Brooks D, Oldstone M, et al.

IL-10 induces aberrant deletion of dendritic cells by natural killer cells in the context of HIV infection

J Clin Invest. 2010; 120(6):1905-13

Amor S, Groome N, Linington C, Morris MM, Dornmair K, Gardinier MV, et al.

Identification of epitopes of myelin oligodendrocyte glycoprotein for the induction of experimental allergic encephalomyelitis in SJL and Biozzi AB/H mice

J Immunol. 1994; 153(10):4349-56

Ando DG, Clayton J, Kono D, Urban JL, Sercarz EE

Encephalitogenic T cells in the B10.PL model of experimental allergic encephalomyelitis (EAE) are of the Th-1 lymphokine subtype

Cell Immunol. 1989; 124(1):132-43

Androdias G, Reynolds R, Chanal M, Ritleng C, Confavreux C, Nataf S

Meningeal $T$ cells associate with diffuse axonal loss in multiple sclerosis spinal cords

Ann Neurol. 2010; 68(4):465-76

Aramaki T, Ida H, Izumi Y, Fujikawa K, Huang M, Arima K, et al.

A significantly impaired natural killer cell activity due to a low activity on a per-cell basis in rheumatoid arthritis

Mod Rheumatol. 2009; 19(3):245-52 
Ardolino M, Zingoni A, Cerboni C, Cecere F, Soriani A, lannitto ML, et al.

DNAM-1 ligand expression on Ag-stimulated T lymphocytes is mediated by ROS-dependent activation of DNA-damage response: relevance for NK-T cell interaction

Blood. 2011; 117(18):4778-86

Ascherio A, Munger KL

Environmental risk factors for multiple sclerosis. Part II: Noninfectious factors

Ann Neurol. 2007; 61(6):504-13

Babbe H, Roers A, Waisman A, Lassmann H, Goebels N, Hohlfeld R, et al.

Clonal expansions of CD8(+) $\mathrm{T}$ cells dominate the $\mathrm{T}$ cell infiltrate in active multiple sclerosis lesions as shown by micromanipulation and single cell polymerase chain reaction

J Exp Med. 2000; 192(3):393-404

Bagnato F, Gupta S, Richert ND, Stone RD, Ohayon JM, Frank JA, et al.

Effects of interferon beta-1b on black holes in multiple sclerosis over a 6-year period with monthly evaluations

Arch Neurol. 2005; 62(11):1684-8

Baron JL, Madri JA, Ruddle NH, Hashim G, Janeway CA, Jr.

Surface expression of alpha 4 integrin by CD4 T cells is required for their entry into brain parenchyma

J Exp Med. 1993; 177(1):57-68

Bauer S, Groh V, Wu J, Steinle A, Phillips JH, Lanier LL, et al.

Activation of NK cells and T cells by NKG2D, a receptor for stress-inducible MICA

Science. 1999; 285(5428):727-9

Becher B, Durell BG, Noelle RJ

Experimental autoimmune encephalitis and inflammation in the absence of interleukin-12

J Clin Invest. 2002; 110(4):493-7

Ben-Nun A, Wekerle H, Cohen IR

The rapid isolation of clonable antigen-specific $\mathrm{T}$ lymphocyte lines capable of mediating autoimmune encephalomyelitis

Eur J Immunol. 1981; 11(3):195-9

Benczur M, Petranyl GG, Palffy G, Varga M, Talas M, Kotsy B, et al.

Dysfunction of natural killer cells in multiple sclerosis: a possible pathogenetic factor

Clin Exp Immunol. 1980; 39(3):657-62

Bengtsson M, Simonsson B, Carlsson K, Nilsson B, Smedmyr B, Termander B, et al

Stimulation of NK cell, $T$ cell, and monocyte functions by the novel immunomodulator Linomide after autologous bone marrow transplantation. A pilot study in patients with acute myeloid leukemia Transplantation. 1992; 53(4):882-8

Benoit LA, Shannon J, Chamberlain JW, Miller RG

Influence of xenogeneic beta2-microglobulin on functional recognition of $\mathrm{H}-2 \mathrm{~Kb}$ by the NK cell inhibitory receptor Ly49C

J Immunol. 2005; 175(6):3542-53

Berer K, Mues M, Koutrolos M, Rasbi ZA, Boziki M, Johner C, et al.

Commensal microbiota and myelin autoantigen cooperate to trigger autoimmune demyelination Nature. 2011; 479(7374):538-41

Bettelli E, Pagany M, Weiner HL, Linington C, Sobel RA, Kuchroo VK

Myelin oligodendrocyte glycoprotein-specific $\mathrm{T}$ cell receptor transgenic mice develop spontaneous autoimmune optic neuritis

J Exp Med. 2003; 197(9):1073-81 
Bettelli E, Sullivan B, Szabo SJ, Sobel RA, Glimcher LH, Kuchroo VK

Loss of T-bet, but not STAT1, prevents the development of experimental autoimmune encephalomyelitis J Exp Med. 2004; 200(1):79-87

Bettencourt A, Silva AM, Carvalho C, Leal B, Santos E, Costa PP, et al. The role of KIR2DS1 in multiple sclerosis: KIR in Portuguese MS patients Journal of Neuroimmunology. 2014; Epub ahead of print

Bielekova B, Catalfamo M, Reichert-Scrivner S, Packer A, Cerna M, Waldmann TA, et al.

Regulatory CD56(bright) natural killer cells mediate immunomodulatory effects of IL-2Ralpha-targeted therapy (daclizumab) in multiple sclerosis

Proc Natl Acad Sci U S A. 2006; 103(15):5941-6

Bielekova B, Richert N, Herman ML, Ohayon J, Waldmann TA, McFarland H, et al.

Intrathecal effects of daclizumab treatment of multiple sclerosis

Neurology. 2011; 77(21):1877-86

Bielekova B, Sung MH, Kadom N, Simon R, McFarland H, Martin R

Expansion and functional relevance of high-avidity myelin-specific CD4+ $\mathrm{T}$ cells in multiple sclerosis

J Immunol. 2004; 172(6):3893-904

Bitsch A, Schuchardt J, Bunkowski S, Kuhlmann T, Brück W

Acute axonal injury in multiple sclerosis. Correlation with demyelination and inflammation

Brain. 2000; 123 ( Pt 6):1174-83

Bonnotte B, Larmonier N, Favre N, Fromentin A, Moutet M, Martin M, et al.

Identification of tumor-infiltrating macrophages as the killers of tumor cells after immunization in a rat model system

J Immunol. 2001; 167(9):5077-83

Bottino C, Castriconi R, Pende D, Rivera P, Nanni M, Carnemolla B, et al.

Identification of PVR (CD155) and Nectin-2 (CD112) as cell surface ligands for the human DNAM-1 (CD226) activating molecule

J Exp Med. 2003; 198(4):557-67

Bourquin C, Schubart A, Tobollik S, Mather I, Ogg S, Liblau R, et al.

Selective unresponsiveness to conformational B cell epitopes of the myelin oligodendrocyte glycoprotein in $\mathrm{H}-2 \mathrm{~b}$ mice

J Immunol. 2003; 171(1):455-61

Brinkmann V, Davis MD, Heise CE, Albert R, Cottens S, Hof R, et al.

The immune modulator FTY720 targets sphingosine 1-phosphate receptors

J Biol Chem. 2002; 277(24):21453-7

Brocke S, Piercy C, Steinman L, Weissman IL, Veromaa T

Antibodies to CD44 and integrin alpha4, but not L-selectin, prevent central nervous system inflammation and experimental encephalomyelitis by blocking secondary leukocyte recruitment

Proc Natl Acad Sci U S A. 1999; 96(12):6896-901

Brück W, Pförtner R, Pham T, Zhang J, Hayardeny L, Piryatinsky V, et al.

Reduced astrocytic NF-kappaB activation by laquinimod protects from cuprizone-induced demyelination Acta Neuropathol. 2012; 124(3):411-24

Brück W, Porada P, Poser S, Rieckmann P, Hanefeld F, Kretzschmar HA, et al.

Monocyte/macrophage differentiation in early multiple sclerosis lesions

Ann Neurol. 1995; 38(5):788-96 
Brück W, Stadelmann C

The spectrum of multiple sclerosis: new lessons from pathology

Curr Opin Neurol. 2005; 18(3):221-4

Brück W, Wegner C

Insight into the mechanism of laquinimod action

J Neurol Sci. 2011; 306(1-2):173-9

Brunmark C, Runstrom A, Ohlsson L, Sparre B, Brodin T, Astrom M, et al.

The new orally active immunoregulator laquinimod (ABR-215062) effectively inhibits development and relapses of experimental autoimmune encephalomyelitis

J Neuroimmunol. 2002; 130(1-2):163-72

Buc M

Role of regulatory $\mathrm{T}$ cells in pathogenesis and biological therapy of multiple sclerosis

Mediators Inflamm. 2013; 2013:963748

Campbell GR, Ziabreva I, Reeve AK, Krishnan KJ, Reynolds R, Howell O, et al. Mitochondrial DNA deletions and neurodegeneration in multiple sclerosis Ann Neurol. 2011; 69(3):481-92

Carbone E, Terrazzano G, Ruggiero G, Zanzi D, Ottaiano A, Manzo C, et al. Recognition of autologous dendritic cells by human NK cells

Eur J Immunol. 1999; 29(12):4022-9

Cerboni C, Zingoni A, Cippitelli M, Piccoli M, Frati L, Santoni A

Antigen-activated human T lymphocytes express cell-surface NKG2D ligands via an ATM/ATR-dependent mechanism and become susceptible to autologous NK- cell lysis

Blood. 2007; 110(2):606-15

Chambers BJ, Salcedo M, Ljunggren HG

Triggering of natural killer cells by the costimulatory molecule CD80 (B7-1)

Immunity. 1996; 5(4):311-7

Chan CJ, Andrews DM, McLaughlin NM, Yagita H, Gilfillan S, Colonna M, et al.

DNAM-1/CD155 interactions promote cytokine and NK cell-mediated suppression of poorly immunogenic melanoma metastases

J Immunol. 2010; 184(2):902-11

Cherwinski HM, Cohn RG, Cheung P, Webster DJ, Xu YZ, Caulfield JP, et al.

The immunosuppressant leflunomide inhibits lymphocyte proliferation by inhibiting pyrimidine biosynthesis

J Pharmacol Exp Ther. 1995; 275(2):1043-9

Chiossone L, Chaix J, Fuseri N, Roth C, Vivier E, Walzer T

Maturation of mouse NK cells is a 4-stage developmental program

Blood. 2009; 113(22):5488-96

Choi JW, Gardell SE, Herr DR, Rivera R, Lee CW, Noguchi K, et al.

FTY720 (fingolimod) efficacy in an animal model of multiple sclerosis requires astrocyte sphingosine 1phosphate receptor 1 (S1P1) modulation

Proc Natl Acad Sci U S A. 2011; 108(2):751-6

Cohen JA, Coles AJ, Arnold DL, Confavreux C, Fox EJ, Hartung HP, et al.

Alemtuzumab versus interferon beta $1 \mathrm{a}$ as first-line treatment for patients with relapsing-remitting multiple sclerosis: a randomised controlled phase 3 trial

Lancet. 2012; 380(9856):1819-28 
Coles AJ, Compston DA, Selmaj KW, Lake SL, Moran S, Margolin DH, et al.

Alemtuzumab vs. interferon beta-1a in early multiple sclerosis

N Engl J Med. 2008; 359(17):1786-801

Coles MC, McMahon CW, Takizawa H, Raulet DH

Memory CD8 T lymphocytes express inhibitory MHC-specific Ly49 receptors

Eur J Immunol. 2000; 30(1):236-44

Comi G, Abramsky O, Arbizu T, Boyko A, Gold R, Havrdova E, et al.

Oral laquinimod in patients with relapsing-remitting multiple sclerosis: 36-week double-blind active extension of the multi-centre, randomized, double-blind, parallel-group placebo-controlled study

Mult Scler. 2010; 16(11):1360-6

Comi G, Filippi M, Wolinsky JS

European/Canadian multicenter, double-blind, randomized, placebo-controlled study of the effects of glatiramer acetate on magnetic resonance imaging--measured disease activity and burden in patients with relapsing multiple sclerosis. European/Canadian Glatiramer Acetate Study Group

Ann Neurol. 2001; 49(3):290-7

Comi G, Jeffery D, Kappos L, Montalban X, Boyko A, Rocca MA, et al.

Placebo-controlled trial of oral laquinimod for multiple sclerosis

N Engl J Med. 2012; 366(11):1000-9

Comi G, Pulizzi A, Rovaris M, Abramsky O, Arbizu T, Boiko A, et al.

Effect of laquinimod on MRI-monitored disease activity in patients with relapsing-remitting multiple sclerosis: a multicentre, randomised, double-blind, placebo-controlled phase Ilb study

Lancet. 2008; 371(9630):2085-92

Confavreux C, Hutchinson M, Hours MM, Cortinovis-Tourniaire P, Moreau T

Rate of pregnancy-related relapse in multiple sclerosis. Pregnancy in Multiple Sclerosis Group

N Engl J Med. 1998; 339(5):285-91

Cooper MA, Fehniger TA, Caligiuri MA

The biology of human natural killer-cell subsets

Trends Immunol. 2001; 22(11):633-40

Cooper MA, Fehniger TA, Turner SC, Chen KS, Ghaheri BA, Ghayur T, et al.

Human natural killer cells: a unique innate immunoregulatory role for the CD56(bright) subset

Blood. 2001; 97(10):3146-51

Corral L, Takizawa H, Hanke T, Jamieson AM, Raulet DH

A new monoclonal antibody reactive with several Ly49 NK cell receptors mediates redirected lysis of target cells

Hybridoma. 1999; 18(4):359-66

Cosman D, Mullberg J, Sutherland CL, Chin W, Armitage R, Fanslow W, et al.

ULBPs, novel MHC class I-related molecules, bind to CMV glycoprotein UL16 and stimulate NK cytotoxicity through the NKG2D receptor

Immunity. 2001; 14(2):123-33

Cua DJ, Sherlock J, Chen Y, Murphy CA, Joyce B, Seymour B, et al.

Interleukin-23 rather than interleukin-12 is the critical cytokine for autoimmune inflammation of the brain

Nature. 2003; 421(6924):744-8 
Cui F, Ji J, Lv H, Qu D, Yu C, Yang Y, et al.

Immune responsiveness in a mouse model of combined adoptive immunotherapy with NK and dendritic cells

J Cancer Res Ther. 2013; 9 Suppl:S162-8

Dan HC, Cooper MJ, Cogswell PC, Duncan JA, Ting JP, Baldwin AS

Akt-dependent regulation of NF-\{kappa\}B is controlled by $\mathrm{mTOR}$ and Raptor in association with IKK

Genes Dev. 2008; 22(11):1490-500

Dardalhon V, Schubart AS, Reddy J, Meyers JH, Monney L, Sabatos CA, et al.

CD226 is specifically expressed on the surface of Th1 cells and regulates their expansion and effector functions

J Immunol. 2005; 175(3):1558-65

de Andrade LF, Smyth MJ, Martinet L

DNAM-1 control of natural killer cells functions through nectin and nectin-like proteins

Immunol Cell Biol. 2013;

De Jager PL, Rossin E, Pyne S, Tamayo P, Ottoboni L, Viglietta V, et al.

Cytometric profiling in multiple sclerosis uncovers patient population structure and a reduction of CD8low cells

Brain. 2008; 131(Pt 7):1701-11

de Matos CT, Berg L, Michaelsson J, Fellander-Tsai L, Karre K, Soderstrom K

Activating and inhibitory receptors on synovial fluid natural killer cells of arthritis patients: role of CD94/NKG2A in control of cytokine secretion

Immunology. 2007; 122(2):291-301

De Stefano N, Matthews PM, Filippi M, Agosta F, De Luca M, Bartolozzi ML, et al.

Evidence of early cortical atrophy in MS: relevance to white matter changes and disability

Neurology. 2003; 60(7):1157-62

Dean G, Kurtzke JF

On the risk of multiple sclerosis according to age at immigration to South Africa

Br Med J. 1971; 3(5777):725-9

Della Chiesa M, Vitale M, Carlomagno S, Ferlazzo G, Moretta L, Moretta A

The natural killer cell-mediated killing of autologous dendritic cells is confined to a cell subset expressing CD94/NKG2A, but lacking inhibitory killer Ig-like receptors

Eur J Immunol. 2003; 33(6):1657-66

Deniz G, Erten G, Kucuksezer UC, Kocacik D, Karagiannidis C, Aktas E, et al.

Regulatory NK cells suppress antigen-specific $T$ cell responses

J Immunol. 2008; 180(2):850-7

Derfuss T, Kappos L

Predicting PML in natalizumab-treated patients: can we do better?

J Neurol Neurosurg Psychiatry. 2013; 84(11):1182-3

Diefenbach A, Jamieson AM, Liu SD, Shastri N, Raulet DH

Ligands for the murine NKG2D receptor: expression by tumor cells and activation of NK cells and macrophages

Nat Immunol. 2000; 1(2):119-26

Dotta F, Censini S, van Halteren AG, Marselli L, Masini M, Dionisi S, et al.

Coxsackie B4 virus infection of beta cells and natural killer cell insulitis in recent-onset type 1 diabetic patients

Proc Natl Acad Sci U S A. 2007; 104(12):5115-20 
Durelli L, Conti L, Clerico M, Boselli D, Contessa G, Ripellino P, et al.

T-helper 17 cells expand in multiple sclerosis and are inhibited by interferon-beta

Ann Neurol. 2009; 65(5):499-509

Dutta R, McDonough J, Yin X, Peterson J, Chang A, Torres T, et al.

Mitochondrial dysfunction as a cause of axonal degeneration in multiple sclerosis patients

Ann Neurol. 2006; 59(3):478-89

Dziedzic T, Metz I, Dallenga T, Konig FB, Muller S, Stadelmann C, et al.

Wallerian degeneration: a major component of early axonal pathology in multiple sclerosis

Brain Pathol. 2010; 20(5):976-85

Edan G, Comi G, Le Page E, Leray E, Rocca MA, Filippi M, et al.

Mitoxantrone prior to interferon beta-1b in aggressive relapsing multiple sclerosis: a 3-year randomised trial

J Neurol Neurosurg Psychiatry. 2011; 82(12):1344-50

Engelhardt B, Diamantstein T, Wekerle H

Immunotherapy of experimental autoimmune encephalomyelitis (EAE): differential effect of anti-IL-2 receptor antibody therapy on actively induced and T-line mediated EAE of the Lewis rat

J Autoimmun. 1989; 2(1):61-73

Evangelou N, Esiri MM, Smith S, Palace J, Matthews PM

Quantitative pathological evidence for axonal loss in normal appearing white matter in multiple sclerosis Ann Neurol. 2000; 47(3):391-5

Evangelou N, Konz D, Esiri MM, Smith S, Palace J, Matthews PM

Regional axonal loss in the corpus callosum correlates with cerebral white matter lesion volume and distribution in multiple sclerosis

Brain. 2000; 123 ( Pt 9):1845-9

Fauriat C, Long EO, Ljunggren HG, Bryceson YT

Regulation of human NK-cell cytokine and chemokine production by target cell recognition

Blood. 2010; 115(11):2167-76

Ferber IA, Brocke S, Taylor-Edwards C, Ridgway W, Dinisco C, Steinman L, et al.

Mice with a disrupted IFN-gamma gene are susceptible to the induction of experimental autoimmune encephalomyelitis (EAE)

J Immunol. 1996; 156(1):5-7

Ferlazzo G, Morandi B, D'Agostino A, Meazza R, Melioli G, Moretta A, et al.

The interaction between NK cells and dendritic cells in bacterial infections results in rapid induction of NK cell activation and in the lysis of uninfected dendritic cells

Eur J Immunol. 2003; 33(2):306-13

Ferlazzo G, Semino C, Melioli G

HLA class I molecule expression is up-regulated during maturation of dendritic cells, protecting them from natural killer cell-mediated lysis

Immunol Lett. 2001; 76(1):37-41

Ferlazzo G, Tsang ML, Moretta L, Melioli G, Steinman RM, Munz C

Human dendritic cells activate resting natural killer (NK) cells and are recognized via the NKp30 receptor by activated NK cells

J Exp Med. 2002; 195(3):343-51 
Fernandez-Morera JL, Rodriguez-Rodero S, Lahoz C, Tunon A, Astudillo A, Garcia-Suarez O, et al. Soluble MHC class I chain-related protein B serum levels correlate with disease activity in relapsingremitting multiple sclerosis

Hum Immunol. 2008; 69(4-5):235-40

Fidler IJ

Selection of successive tumour lines for metastasis

Nat New Biol. 1973; 242(118):148-9

Fidler IJ

Biological behavior of malignant melanoma cells correlated to their survival in vivo

Cancer Res. 1975; 35(1):218-24

Filippi M, Rocca MA, Pagani E, De Stefano N, Jeffery D, Kappos L, et al.

Placebo-controlled trial of oral laquinimod in multiple sclerosis: MRI evidence of an effect on brain tissue damage

J Neurol Neurosurg Psychiatry. 2013;

Filippi M, Rovaris M, Inglese M, Barkhof F, De Stefano N, Smith S, et al.

Interferon beta-1a for brain tissue loss in patients at presentation with syndromes suggestive of multiple sclerosis: a randomised, double-blind, placebo-controlled trial

Lancet. 2004; 364(9444):1489-96

Fisher E, Lee JC, Nakamura K, Rudick RA

Gray matter atrophy in multiple sclerosis: a longitudinal study

Ann Neurol. 2008; 64(3):255-65

Ford ML, Evavold BD

Specificity, magnitude, and kinetics of MOG-specific CD8+ T cell responses during experimental autoimmune encephalomyelitis

Eur J Immunol. 2005; 35(1):76-85

Fortin C, Huang $X$, Yang $Y$

Both NK cell-intrinsic and -extrinsic STAT1 signaling are required for NK cell response against vaccinia virus

J Immunol. 2013; 191(1):363-8

Fox RJ, Miller DH, Phillips JT, Hutchinson M, Havrdova E, Kita M, et al.

Placebo-controlled phase 3 study of oral BG-12 or glatiramer in multiple sclerosis

N Engl J Med. 2012; 367(12):1087-97

Fridkis-Hareli M, Teitelbaum D, Gurevich E, Pecht I, Brautbar C, Kwon OJ, et al.

Direct binding of myelin basic protein and synthetic copolymer 1 to class II major histocompatibility complex molecules on living antigen-presenting cells--specificity and promiscuity

Proc Natl Acad Sci U S A. 1994; 91(11):4872-6

Fu B, Wang F, Sun R, Ling B, Tian Z, Wei H

CD11b and CD27 reflect distinct population and functional specialization in human natural killer cells Immunology. 2011; 133(3):350-9

Fuchs A, Cella M, Giurisato E, Shaw AS, Colonna M

Cutting edge: CD96 (tactile) promotes NK cell-target cell adhesion by interacting with the poliovirus receptor (CD155)

J Immunol. 2004; 172(7):3994-8 
Gan Y, Liu R, Wu W, Bomprezzi R, Shi FD

Antibody to alpha4 integrin suppresses natural killer cells infiltration in central nervous system in experimental autoimmune encephalomyelitis

J Neuroimmunol. 2012; 247(1-2):9-15

Ganal SC, Sanos SL, Kallfass C, Oberle K, Johner C, Kirschning C, et al.

Priming of natural killer cells by nonmucosal mononuclear phagocytes requires instructive signals from commensal microbiota

Immunity. 2012; 37(1):171-86

Gandhi R, Laroni A, Weiner HL

Role of the innate immune system in the pathogenesis of multiple sclerosis

J Neuroimmunol. 2010; 221(1-2):7-14

Gerosa F, Baldani-Guerra B, Nisii C, Marchesini V, Carra G, Trinchieri G

Reciprocal activating interaction between natural killer cells and dendritic cells

J Exp Med. 2002; 195(3):327-33

Ghoreschi K, Bruck J, Kellerer C, Deng C, Peng H, Rothfuss O, et al.

Fumarates improve psoriasis and multiple sclerosis by inducing type II dendritic cells

J Exp Med. 2011; 208(11):2291-303

Gilfillan S, Chan CJ, Cella M, Haynes NM, Rapaport AS, Boles KS, et al.

DNAM-1 promotes activation of cytotoxic lymphocytes by nonprofessional antigen-presenting cells and tumors

J Exp Med. 2008; 205(13):2965-73

Gilmore CP, Donaldson I, Bo L, Owens T, Lowe J, Evangelou N

Regional variations in the extent and pattern of grey matter demyelination in multiple sclerosis: a comparison between the cerebral cortex, cerebellar cortex, deep grey matter nuclei and the spinal cord J Neurol Neurosurg Psychiatry. 2009; 80(2):182-7

Godfrey DI, Stankovic S, Baxter AG

Raising the NKT cell family

Nat Immunol. 2010; 11(3):197-206

Gold R, Kappos L, Arnold DL, Bar-Or A, Giovannoni G, Selmaj K, et al.

Placebo-controlled phase 3 study of oral BG-12 for relapsing multiple sclerosis

N Engl J Med. 2012; 367(12):1098-107

Gold R, Linington C, Lassmann $\mathrm{H}$

Understanding pathogenesis and therapy of multiple sclerosis via animal models: $\mathbf{7 0}$ years of merits and culprits in experimental autoimmune encephalomyelitis research

Brain. 2006; 129(Pt 8):1953-71

Goldschmidt T, Antel J, Konig FB, Brück W, Kuhlmann T

Remyelination capacity of the MS brain decreases with disease chronicity

Neurology. 2009; 72(22):1914-21

Greene S, Watanabe K, Braatz-Trulson J, Lou L

Inhibition of dihydroorotate dehydrogenase by the immunosuppressive agent leflunomide

Biochem Pharmacol. 1995; 50(6):861-7

Groh V, Wu J, Yee C, Spies T

Tumour-derived soluble MIC ligands impair expression of NKG2D and T-cell activation

Nature. 2002; 419(6908):734-8 
Haak S, Croxford AL, Kreymborg K, Heppner FL, Pouly S, Becher B, et al.

IL-17A and IL-17F do not contribute vitally to autoimmune neuro-inflammation in mice

J Clin Invest. 2009; 119(1):61-9

Haas J, Hug A, Viehover A, Fritzsching B, Falk CS, Filser A, et al.

Reduced suppressive effect of $C D 4+C D 25$ high regulatory $T$ cells on the $T$ cell immune response against myelin oligodendrocyte glycoprotein in patients with multiple sclerosis

Eur J Immunol. 2005; 35(11):3343-52

Hafler DA, Duby AD, Lee SJ, Benjamin D, Seidman JG, Weiner HL

Oligoclonal $\mathrm{T}$ lymphocytes in the cerebrospinal fluid of patients with multiple sclerosis

J Exp Med. 1988; 167(4):1313-22

Hafler JP, Maier LM, Cooper JD, Plagnol V, Hinks A, Simmonds MJ, et al.

CD226 Gly307Ser association with multiple autoimmune diseases

Genes Immun. 2009; 10(1):5-10

Hammarberg H, Lidman O, Lundberg C, Eltayeb SY, Gielen AW, Muhallab S, et al.

Neuroprotection by encephalomyelitis: rescue of mechanically injured neurons and neurotrophin production by CNS-infiltrating $\mathrm{T}$ and natural killer cells

The Journal of neuroscience : the official journal of the Society for Neuroscience. 2000; 20(14):5283-91

Hansen T, Skytthe A, Stenager E, Petersen HC, Bronnum-Hansen H, Kyvik KO

Concordance for multiple sclerosis in Danish twins: an update of a nationwide study

Mult Scler. 2005; 11(5):504-10

Hartung HP, Gonsette R, Konig N, Kwiecinski H, Guseo A, Morrissey SP, et al.

Mitoxantrone in progressive multiple sclerosis: a placebo-controlled, double-blind, randomised, multicentre trial

Lancet. 2002; 360(9350):2018-25

Hauser SL, Ault KA, Levin MJ, Garovoy MR, Weiner HL

Natural killer cell activity in multiple sclerosis

J Immunol. 1981; 127(3):1114-7

Hauser SL, Waubant E, Arnold DL, Vollmer T, Antel J, Fox RJ, et al.

B-cell depletion with rituximab in relapsing-remitting multiple sclerosis

N Engl J Med. 2008; 358(7):676-88

Hayakawa Y, Huntington ND, Nutt SL, Smyth MJ

Functional subsets of mouse natural killer cells

Immunol Rev. 2006; 214:47-55

Hayakawa Y, Screpanti V, Yagita H, Grandien A, Ljunggren HG, Smyth MJ, et al.

NK cell TRAIL eliminates immature dendritic cells in vivo and limits dendritic cell vaccination efficacy

J Immunol. 2004; 172(1):123-9

Hayosh NS, Swanborg RH

Autoimmune effector cells. IX. Inhibition of adoptive transfer of autoimmune encephalomyelitis with a monoclonal antibody specific for interleukin 2 receptors

J Immunol. 1987; 138(11):3771-5

Healy BC, Ali EN, Guttmann CR, Chitnis T, Glanz BI, Buckle G, et al.

Smoking and disease progression in multiple sclerosis

Arch Neurol. 2009; 66(7):858-64 
Hedstrom AK, Baarnhielm M, Olsson T, Alfredsson L

Tobacco smoking, but not Swedish snuff use, increases the risk of multiple sclerosis

Neurology. 2009; 73(9):696-701

Hein T, Hopfenmuller W

[Projection of the number of multiple sclerosis patients in Germany]

Nervenarzt. 2000; 71(4):288-94

Hernan MA, Olek MJ, Ascherio A

Cigarette smoking and incidence of multiple sclerosis

Am J Epidemiol. 2001; 154(1):69-74

Hewer S, Lucas R, van der Mei I, Taylor BV

Vitamin $D$ and multiple sclerosis

J Clin Neurosci. 2013; 20(5):634-41

Hofstetter HH, Ibrahim SM, Koczan D, Kruse N, Weishaupt A, Toyka KV, et al.

Therapeutic efficacy of IL-17 neutralization in murine experimental autoimmune encephalomyelitis

Cell Immunol. 2005; 237(2):123-30

Hoftberger R, Fink S, Aboul-Enein F, Botond G, Olah J, Berki T, et al.

Tubulin polymerization promoting protein (TPPP/p25) as a marker for oligodendroglial changes in multiple sclerosis

Glia. 2010; 58(15):1847-57

Hori S, Nomura T, Sakaguchi S

Control of regulatory $\mathrm{T}$ cell development by the transcription factor Foxp3

Science. 2003; 299(5609):1057-61

Howell OW, Rundle JL, Garg A, Komada M, Brophy PJ, Reynolds R

Activated microglia mediate axoglial disruption that contributes to axonal injury in multiple sclerosis

J Neuropathol Exp Neurol. 2010; 69(10):1017-33

Hu Y, Ota N, Peng I, Refino CJ, Danilenko DM, Caplazi P, et al.

IL-17RC is required for IL-17A- and IL-17F-dependent signaling and the pathogenesis of experimental autoimmune encephalomyelitis

J Immunol. 2010; 184(8):4307-16

Huang D, Shi FD, Jung S, Pien GC, Wang J, Salazar-Mather TP, et al.

The neuronal chemokine $\mathrm{CX} 3 \mathrm{CL} 1 /$ fractalkine selectively recruits NK cells that modify experimental autoimmune encephalomyelitis within the central nervous system

FASEB J. 2006; 20(7):896-905

Huang Z, Fu B, Zheng SG, Li X, Sun R, Tian Z, et al.

Involvement of CD226+ NK cells in immunopathogenesis of systemic lupus erythematosus

J Immunol. 2011; 186(6):3421-31

Huseby ES, Liggitt D, Brabb T, Schnabel B, Ohlen C, Goverman J

A pathogenic role for myelin-specific $\mathrm{CD} 8(+) \mathrm{T}$ cells in a model for multiple sclerosis

J Exp Med. 2001; 194(5):669-76

Jacobs LD, Beck RW, Simon JH, Kinkel RP, Brownscheidle CM, Murray TJ, et al.

Intramuscular interferon beta-1a therapy initiated during a first demyelinating event in multiple sclerosis. CHAMPS Study Group

N Engl J Med. 2000; 343(13):898-904 
Jacobsen M, Cepok S, Quak E, Happel M, Gaber R, Ziegler A, et al.

Oligoclonal expansion of memory CD8+ T cells in cerebrospinal fluid from multiple sclerosis patients Brain. 2002; 125(Pt 3):538-50

Jäger A, Dardalhon V, Sobel RA, Bettelli E, Kuchroo VK

Th1, Th17, and Th9 effector cells induce experimental autoimmune encephalomyelitis with different pathological phenotypes

J Immunol. 2009; 183(11):7169-77

Ji Q, Perchellet A, Goverman JM

Viral infection triggers central nervous system autoimmunity via activation of CD8+ $T$ cells expressing dual TCRs

Nat Immunol. 2010; 11(7):628-34

Johnson KP, Brooks BR, Cohen JA, Ford CC, Goldstein J, Lisak RP, et al.

Copolymer 1 reduces relapse rate and improves disability in relapsing-remitting multiple sclerosis: results of a phase III multicenter, double-blind placebo-controlled trial. The Copolymer 1 Multiple Sclerosis Study Group Neurology. 1995; 45(7):1268-76

Johnson TA, Evans BL, Durafourt BA, Blain M, Lapierre Y, Bar-Or A, et al.

Reduction of the peripheral blood CD56(bright) NK lymphocyte subset in FTY720-treated multiple sclerosis patients

J Immunol. 2011; 187(1):570-9

Jolivel V, Luessi F, Masri J, Kraus SH, Hubo M, Poisa-Beiro L, et al.

Modulation of dendritic cell properties by laquinimod as a mechanism for modulating multiple sclerosis Brain. 2013; 136(Pt 4):1048-66

Joller N, Hafler JP, Brynedal B, Kassam N, Spoerl S, Levin SD, et al.

Cutting edge: TIGIT has T cell-intrinsic inhibitory functions

J Immunol. 2011; 186(3):1338-42

Jönsson S, Andersson G, Fex T, Fristedt T, Hedlund G, Jansson K, et al.

Synthesis and biological evaluation of new 1,2-dihydro-4-hydroxy-2-oxo-3-quinolinecarboxamides for treatment of autoimmune disorders: structure-activity relationship

J Med Chem. 2004; 47(8):2075-88

Kalland T

Effects of the immunomodulator LS 2616 on growth and metastasis of the murine B16-F10 melanoma

Cancer Res. 1986; 46(6):3018-22

Kalland T, Alm G, Stalhandshe T

Augmentation of mouse natural killer cell activity by LS 2616, a new immunomodulator

J Immunol. 1985; 134(6):3956-61

Kamath AT, Pooley J, O'Keeffe MA, Vremec D, Zhan Y, Lew AM, et al.

The development, maturation, and turnover rate of mouse spleen dendritic cell populations

J Immunol. 2000; 165(12):6762-70

Kappos L, Antel J, Comi G, Montalban X, O'Connor P, Polman CH, et al.

Oral fingolimod (FTY720) for relapsing multiple sclerosis

N Engl J Med. 2006; 355(11):1124-40

Kappos L, Freedman MS, Polman CH, Edan G, Hartung HP, Miller DH, et al

Effect of early versus delayed interferon beta-1b treatment on disability after a first clinical event suggestive of multiple sclerosis: a 3-year follow-up analysis of the BENEFIT study

Lancet. 2007; 370(9585):389-97 
Kappos L, Radue EW, O'Connor P, Polman C, Hohlfeld R, Calabresi P, et al. A placebo-controlled trial of oral fingolimod in relapsing multiple sclerosis N Engl J Med. 2010; 362(5):387-401

Karni A, Abraham M, Monsonego A, Cai G, Freeman GJ, Hafler D, et al. Innate immunity in multiple sclerosis: myeloid dendritic cells in secondary progressive multiple sclerosis are activated and drive a proinflammatory immune response J Immunol. 2006; 177(6):4196-202

Karussis DM, Lehmann D, Slavin S, Vourka-Karussis U, Mizrachi-Koll R, Ovadia H, et al. Treatment of chronic-relapsing experimental autoimmune encephalomyelitis with the synthetic immunomodulator linomide (quinoline-3-carboxamide)

Proc Natl Acad Sci U S A. 1993; 90(14):6400-4

Kastrukoff LF, Lau A, Wee R, Zecchini D, White R, Paty DW

Clinical relapses of multiple sclerosis are associated with 'novel' valleys in natural killer cell functional activity

J Neuroimmunol. 2003; 145(1-2):103-14

Kastrukoff LF, Morgan NG, Zecchini D, White R, Petkau AJ, Satoh J, et al.

A role for natural killer cells in the immunopathogenesis of multiple sclerosis

J Neuroimmunol. 1998; 86(2):123-33

Kataoka H, Sugahara K, Shimano K, Teshima K, Koyama M, Fukunari A, et al.

FTY720, sphingosine 1-phosphate receptor modulator, ameliorates experimental autoimmune encephalomyelitis by inhibition of $\mathrm{T}$ cell infiltration

Cell Mol Immunol. 2005; 2(6):439-48

Keith AB, Arnon R, Teitelbaum D, Caspary EA, Wisniewski HM

The effect of Cop 1, a synthetic polypeptide, on chronic relapsing experimental allergic encephalomyelitis in guinea pigs

J Neurol Sci. 1979; 42(2):267-74

Kent SJ, Karlik SJ, Cannon C, Hines DK, Yednock TA, Fritz LC, et al.

A monoclonal antibody to alpha 4 integrin suppresses and reverses active experimental allergic encephalomyelitis

J Neuroimmunol. 1995; 58(1):1-10

Keszthelyi E, Karlik S, Hyduk S, Rice GP, Gordon G, Yednock T, et al.

Evidence for a prolonged role of alpha 4 integrin throughout active experimental allergic encephalomyelitis

Neurology. 1996; 47(4):1053-9

Kim S, lizuka K, Kang HS, Dokun A, French AR, Greco S, et al.

In vivo developmental stages in murine natural killer cell maturation

Nat Immunol. 2002; 3(6):523-8

Klotz L, Meuth SG, Wiendl H

Immune mechanisms of new therapeutic strategies in multiple sclerosis-A focus on alemtuzumab Clin Immunol. 2012; 142(1):25-30

Kohm AP, Carpentier PA, Anger HA, Miller SD

Cutting edge: $C D 4+C D 25+$ regulatory $T$ cells suppress antigen-specific autoreactive immune responses and central nervous system inflammation during active experimental autoimmune encephalomyelitis J Immunol. 2002; 169(9):4712-6 
Komiyama Y, Nakae S, Matsuki T, Nambu A, Ishigame H, Kakuta S, et al.

IL-17 plays an important role in the development of experimental autoimmune encephalomyelitis J Immunol. 2006; 177(1):566-73

Koritschoner RS, Schweinburg F

Induktion von Paralyse und Rückenmarksentzündung durch Immunisierung von Kaninchen mit menschlichem Rückenmarksgewebe.

Immunitätsf Exp Ther. 1925; 42:217 - 83

Kowarik MC, Pellkofer HL, Cepok S, Korn T, Kumpfel T, Buck D, et al.

Differential effects of fingolimod (FTY720) on immune cells in the CSF and blood of patients with MS

Neurology. 2011; 76(14):1214-21

Krishnamoorthy G, Lassmann H, Wekerle H, Holz A

Spontaneous opticospinal encephalomyelitis in a double-transgenic mouse model of autoimmune T cell/B cell cooperation

J Clin Invest. 2006; 116(9):2385-92

Krishnamoorthy G, Wekerle H

EAE: an immunologist's magic eye

Eur J Immunol. 2009; 39(8):2031-5

Kroenke MA, Carlson TJ, Andjelkovic AV, Segal BM

IL-12- and IL-23-modulated T cells induce distinct types of EAE based on histology, CNS chemokine profile, and response to cytokine inhibition

J Exp Med. 2008; 205(7):1535-41

Kuchroo VK, Das MP, Brown JA, Ranger AM, Zamvil SS, Sobel RA, et al.

B7-1 and B7-2 costimulatory molecules activate differentially the Th1/Th2 developmental pathways: application to autoimmune disease therapy

Cell. 1995; 80(5):707-18

Kurtzke JF

Epidemiology of multiple sclerosis. Does this really point toward an etiology? Lectio Doctoralis

Neurol Sci. 2000; 21(6):383-403

Kurtzke JF

Multiple sclerosis in time and space--geographic clues to cause

J Neurovirol. 2000; 6 Suppl 2:S134-40

Kutzelnigg A, Lucchinetti CF, Stadelmann C, Brück W, Rauschka H, Bergmann M, et al.

Cortical demyelination and diffuse white matter injury in multiple sclerosis

Brain. 2005; 128(Pt 11):2705-12

Lakshmikanth T, Burke S, Ali TH, Kimpfler S, Ursini F, Ruggeri L, et al.

NCRs and DNAM-1 mediate NK cell recognition and lysis of human and mouse melanoma cell lines in vitro and in vivo

J Clin Invest. 2009; 119(5):1251-63

Langrish CL, Chen Y, Blumenschein WM, Mattson J, Basham B, Sedgwick JD, et al.

IL-23 drives a pathogenic $T$ cell population that induces autoimmune inflammation

J Exp Med. 2005; 201(2):233-40

Lanier LL, Buck DW, Rhodes L, Ding A, Evans E, Barney C, et al.

Interleukin 2 activation of natural killer cells rapidly induces the expression and phosphorylation of the Leu-23 activation antigen

J Exp Med. 1988; 167(5):1572-85 
Lanier LL, Le AM, Civin Cl, Loken MR, Phillips JH

The relationship of CD16 (Leu-11) and Leu-19 (NKH-1) antigen expression on human peripheral blood NK cells and cytotoxic $T$ lymphocytes

J Immunol. 1986; 136(12):4480-6

Leavenworth JW, Schellack C, Kim HJ, Lu L, Spee P, Cantor H

Analysis of the cellular mechanism underlying inhibition of EAE after treatment with anti-NKG2A F(ab')2

Proc Natl Acad Sci U S A. 2010; 107(6):2562-7

Lee CK, Rao DT, Gertner R, Gimeno R, Frey AB, Levy DE

Distinct requirements for IFNs and STAT1 in NK cell function

J Immunol. 2000; 165(7):3571-7

Lee IF, Qin H, Priatel JJ, Tan R

Critical role for IFN-gamma in natural killer cell-mediated protection from diabetes

Eur J Immunol. 2008; 38(1):82-9

Lee IF, Qin H, Trudeau J, Dutz J, Tan R

Regulation of autoimmune diabetes by complete Freund's adjuvant is mediated by NK cells

J Immunol. 2004; 172(2):937-42

Levin SD, Taft DW, Brandt CS, Bucher C, Howard ED, Chadwick EM, et al.

Vstm3 is a member of the CD28 family and an important modulator of T-cell function

Eur J Immunol. 2011; 41(4):902-15

Levine S, Saltzman A

Regional suppression, therapy after onset and prevention of relapses in experimental allergic encephalomyelitis by mitoxantrone

J Neuroimmunol. 1986; 13(2):175-81

Linker RA, Lee DH, Ryan S, van Dam AM, Conrad R, Bista P, et al.

Fumaric acid esters exert neuroprotective effects in neuroinflammation via activation of the Nrf2 antioxidant pathway

Brain. 2011; 134(Pt 3):678-92

Litzenburger T, Fassler R, Bauer J, Lassmann H, Linington C, Wekerle H, et al.

B lymphocytes producing demyelinating autoantibodies: development and function in gene-targeted transgenic mice

J Exp Med. 1998; 188(1):169-80

Liu S, Zhang H, Li M, Hu D, Li C, Ge B, et al.

Recruitment of Grb2 and SHIP1 by the ITT-like motif of TIGIT suppresses granule polarization and cytotoxicity of NK cells

Cell Death Differ. 2013; 20(3):456-64

Liu Y, Teige I, Birnir B, Issazadeh-Navikas S

Neuron-mediated generation of regulatory $T$ cells from encephalitogenic $T$ cells suppresses EAE

Nat Med. 2006; 12(5):518-25

Lodoen M, Ogasawara K, Hamerman JA, Arase H, Houchins JP, Mocarski ES, et al.

NKG2D-mediated natural killer cell protection against cytomegalovirus is impaired by viral gp40 modulation of retinoic acid early inducible 1 gene molecules

J Exp Med. 2003; 197(10):1245-53

Long EO, Kim HS, Liu D, Peterson ME, Rajagopalan S

Controlling natural killer cell responses: integration of signals for activation and inhibition

Annu Rev Immunol. 2013; 31:227-58 
Lozano E, Dominguez-Villar M, Kuchroo V, Hafler DA

The TIGIT/CD226 axis regulates human T cell function

J Immunol. 2012; 188(8):3869-75

Lozano E, Joller N, Cao Y, Kuchroo VK, Hafler DA

The CD226/CD155 interaction regulates the proinflammatory (Th1/Th17)/anti-inflammatory (Th2) balance in humans

J Immunol. 2013; 191(7):3673-80

Lu L, Ikizawa K, Hu D, Werneck MB, Wucherpfennig KW, Cantor H

Regulation of activated CD4+ T cells by NK cells via the Qa-1-NKG2A inhibitory pathway

Immunity. 2007; 26(5):593-604

Lublin FD, Lavasa M, Viti C, Knobler RL

Suppression of acute and relapsing experimental allergic encephalomyelitis with mitoxantrone Clin Immunol Immunopathol. 1987; 45(1):122-8

Lucas RM, Ponsonby AL, Dear K, Valery PC, Pender MP, Taylor BV, et al.

Sun exposure and vitamin $D$ are independent risk factors for CNS demyelination

Neurology. 2011; 76(6):540-8

Lucchinetti C, Brück W, Parisi J, Scheithauer B, Rodriguez M, Lassmann H

Heterogeneity of multiple sclerosis lesions: implications for the pathogenesis of demyelination

Ann Neurol. 2000; 47(6):707-17

Ma DY, Clark EA

The role of CD40 and CD154/CD40L in dendritic cells

Semin Immunol. 2009; 21(5):265-72

Mahad DJ, Ziabreva I, Campbell G, Lax N, White K, Hanson PS, et al.

Mitochondrial changes within axons in multiple sclerosis

Brain. 2009; 132(Pt 5):1161-74

Maier MK, Seth S, Czeloth N, Qiu Q, Ravens I, Kremmer E, et al.

The adhesion receptor CD155 determines the magnitude of humoral immune responses against orally ingested antigens

Eur J Immunol. 2007; 37(8):2214-25

Mandala S, Hajdu R, Bergstrom J, Quackenbush E, Xie J, Milligan J, et al.

Alteration of lymphocyte trafficking by sphingosine-1-phosphate receptor agonists

Science. 2002; 296(5566):346-9

Martin-Fontecha A, Thomsen LL, Brett S, Gerard C, Lipp M, Lanzavecchia A, et al.

Induced recruitment of NK cells to lymph nodes provides IFN-gamma for $T(H) 1$ priming

Nat Immunol. 2004; 5(12):1260-5

Martin JF, Perry JS, Jakhete NR, Wang X, Bielekova B

An IL-2 paradox: blocking CD25 on T cells induces IL-2-driven activation of CD56(bright) NK cells $\mathrm{J}$ Immunol. 2010; 185(2):1311-20

Martinez-Rodriguez JE, Lopez-Botet M, Munteis E, Rio J, Roquer J, Montalban X, et al.

Natural killer cell phenotype and clinical response to interferon-beta therapy in multiple sclerosis

Clin Immunol. 2011; 141(3):348-56

Martinez-Rodriguez JE, Saez-Borderias A, Munteis E, Romo N, Roquer J, Lopez-Botet M

Natural killer receptors distribution in multiple sclerosis: Relation to clinical course and interferon-beta therapy

Clin Immunol. 2010; 137(1):41-50 
McDonald WI, Compston A, Edan G, Goodkin D, Hartung HP, Lublin FD, et al.

Recommended diagnostic criteria for multiple sclerosis: guidelines from the International Panel on the diagnosis of multiple sclerosis

Ann Neurol. 2001; 50(1):121-7

McGeachy MJ, Stephens LA, Anderton SM

Natural recovery and protection from autoimmune encephalomyelitis: contribution of CD4+CD25+ regulatory cells within the central nervous system

J Immunol. 2005; 175(5):3025-32

Mendel I, Kerlero de Rosbo N, Ben-Nun A

A myelin oligodendrocyte glycoprotein peptide induces typical chronic experimental autoimmune encephalomyelitis in $\mathrm{H}-2 \mathrm{~b}$ mice: fine specificity and $\mathrm{T}$ cell receptor $\mathrm{V}$ beta expression of encephalitogenic T cells

Eur J Immunol. 1995; 25(7):1951-9

Milo R, Kahana E

Multiple sclerosis: geoepidemiology, genetics and the environment

Autoimmun Rev. 2010; 9(5):A387-94

Mishra M, Silva C, Wang J, Yong V

Mechanisms of laquinimod in multiple sclerosis: focus on microglia

29th Congress of the European Committee for Treatment and Research in Multiple Sclerosis (ECTRIMS). 2013:P 572

Mishra MK, Wang J, Silva C, Mack M, Yong VW

Kinetics of proinflammatory monocytes in a model of multiple sclerosis and its perturbation by laquinimod

Am J Pathol. 2012; 181(2):642-51

Mizutani T, Neugebauer N, Putz EM, Moritz N, Simma O, Zebedin-Brandl E, et al.

Conditional IFNAR1 ablation reveals distinct requirements of Type I IFN signaling for NK cell maturation and tumor surveillance

Oncoimmunology. 2012; 1(7):1027-37

Mombaerts P, lacomini J, Johnson RS, Herrup K, Tonegawa S, Papaioannou VE

RAG-1-deficient mice have no mature $B$ and $T$ lymphocytes

Cell. 1992; 68(5):869-77

Montero E, Nussbaum G, Kaye JF, Perez R, Lage A, Ben-Nun A, et al.

Regulation of experimental autoimmune encephalomyelitis by CD4+, CD25+ and CD8+ T cells: analysis using depleting antibodies

J Autoimmun. 2004; 23(1):1-7

Moore S, Khalaj AJ, Yoon J, Patel R, Hannsun G, Yoo T, et al.

Therapeutic laquinimod treatment decreases inflammation, initiates axon remyelination, and improves motor deficit in a mouse model of multiple sclerosis

Brain Behav. 2013; 3(6):664-82

Mumford CJ, Wood NW, Kellar-Wood H, Thorpe JW, Miller DH, Compston DA

The British Isles survey of multiple sclerosis in twins

Neurology. 1994; 44(1):11-5

Munger KL, Levin LI, Hollis BW, Howard NS, Ascherio A

Serum 25-hydroxyvitamin $D$ levels and risk of multiple sclerosis

JAMA. 2006; 296(23):2832-8 
Munger KL, Zhang SM, O'Reilly E, Hernan MA, Olek MJ, Willett WC, et al.

Vitamin $D$ intake and incidence of multiple sclerosis

Neurology. 2004; 62(1):60-5

Munoz-Culla M, Irizar H, Otaegui D

The genetics of multiple sclerosis: review of current and emerging candidates

Appl Clin Genet. 2013; 6:63-73

Munschauer FE, Hartrich LA, Stewart CC, Jacobs L

Circulating natural killer cells but not cytotoxic $\mathrm{T}$ lymphocytes are reduced in patients with active relapsing multiple sclerosis and little clinical disability as compared to controls

J Neuroimmunol. 1995; 62(2):177-81

Na SY, Cao Y, Toben C, Nitschke L, Stadelmann C, Gold R, et al.

Naive CD8 T-cells initiate spontaneous autoimmunity to a sequestered model antigen of the central nervous system

Brain. 2008; 131(Pt 9):2353-65

Najafian N, Chitnis T, Salama AD, Zhu B, Benou C, Yuan X, et al.

Regulatory functions of CD8+CD28- T cells in an autoimmune disease model

J Clin Invest. 2003; 112(7):1037-48

Nakayama M, Takeda K, Kawano M, Takai T, Ishii N, Ogasawara K

Natural killer (NK)-dendritic cell interactions generate MHC class II-dressed NK cells that regulate CD4+ T cells

Proc Natl Acad Sci U S A. 2011; 108(45):18360-5

Natarajan K, Dimasi N, Wang J, Mariuzza RA, Margulies DH

Structure and function of natural killer cell receptors: multiple molecular solutions to self, nonself discrimination

Annu Rev Immunol. 2002; 20:853-85

Neighbour PA, Grayzel Al, Miller AE

Endogenous and interferon-augmented natural killer cell activity of human peripheral blood mononuclear cells in vitro. Studies of patients with multiple sclerosis, systemic lupus erythematosus or rheumatoid arthritis

Clin Exp Immunol. 1982; 49(1):11-21

Neuhaus O, Farina C, Yassouridis A, Wiendl H, Then Bergh F, Dose T, et al.

Multiple sclerosis: comparison of copolymer-1- reactive $T$ cell lines from treated and untreated subjects reveals cytokine shift from $T$ helper 1 to T helper 2 cells

Proc Natl Acad Sci U S A. 2000; 97(13):7452-7

Nguyen KB, Salazar-Mather TP, Dalod MY, Van Deusen JB, Wei XQ, Liew FY, et al.

Coordinated and distinct roles for IFN-alpha beta, IL-12, and IL-15 regulation of NK cell responses to viral infection

J Immunol. 2002; 169(8):4279-87

Nielsen N, Odum N, Urso B, Lanier LL, Spee P

Cytotoxicity of CD56(bright) NK cells towards autologous activated CD4+ T cells is mediated through NKG2D, LFA-1 and TRAIL and dampened via CD94/NKG2A

PLoS One. 2012; 7(2):e31959

Noseworthy JH, Wolinsky JS, Lublin FD, Whitaker JN, Linde A, Gjorstrup P, et al.

Linomide in relapsing and secondary progressive MS: part I: trial design and clinical results. North American Linomide Investigators

Neurology. 2000; 54(9):1726-33 
O'Connor P, Wolinsky JS, Confavreux C, Comi G, Kappos L, Olsson TP, et al. Randomized trial of oral teriflunomide for relapsing multiple sclerosis N Engl J Med. 2011; 365(14):1293-303

O'Connor PW, Li D, Freedman MS, Bar-Or A, Rice GP, Confavreux C, et al.

A Phase II study of the safety and efficacy of teriflunomide in multiple sclerosis with relapses Neurology. 2006; 66(6):894-900

Ochoa-Reparaz J, Mielcarz DW, Ditrio LE, Burroughs AR, Begum-Haque S, Dasgupta S, et al.

Central nervous system demyelinating disease protection by the human commensal Bacteroides fragilis depends on polysaccharide $A$ expression

J Immunol. 2010; 185(7):4101-8

Ochoa-Reparaz J, Mielcarz DW, Ditrio LE, Burroughs AR, Foureau DM, Haque-Begum S, et al. Role of gut commensal microflora in the development of experimental autoimmune encephalomyelitis J Immunol. 2009; 183(10):6041-50

Ochoa-Reparaz J, Mielcarz DW, Haque-Begum S, Kasper LH Induction of a regulatory B cell population in experimental allergic encephalomyelitis by alteration of the gut commensal microflora

Gut Microbes. 2010; 1(2):103-8

Olsson T, Sun J, Hillert J, Hojeberg B, Ekre HP, Andersson G, et al.

Increased numbers of $\mathrm{T}$ cells recognizing multiple myelin basic protein epitopes in multiple sclerosis Eur J Immunol. 1992; 22(4):1083-7

Orange JS, Brodeur SR, Jain A, Bonilla FA, Schneider LC, Kretschmer R, et al.

Deficient natural killer cell cytotoxicity in patients with IKK-gamma/NEMO mutations

J Clin Invest. 2002; 109(11):1501-9

Orr MT, Murphy WJ, Lanier LL

'Unlicensed' natural killer cells dominate the response to cytomegalovirus infection Nat Immunol. 2010; 11(4):321-7

Orr MT, Sun JC, Hesslein DG, Arase H, Phillips JH, Takai T, et al.

Ly49H signaling through DAP10 is essential for optimal natural killer cell responses to mouse cytomegalovirus infection

J Exp Med. 2009; 206(4):807-17

Ortaldo JR, Winkler-Pickett R, Wiegand G

Activating Ly-49D NK receptors: expression and function in relation to ontogeny and Ly-49 inhibitor receptors

J Leukoc Biol. 2000; 68(5):748-56

Orton SM, Ramagopalan SV, Brocklebank D, Herrera BM, Dyment DA, Yee IM, et al.

Effect of immigration on multiple sclerosis sex ratio in Canada: the Canadian Collaborative Study

J Neurol Neurosurg Psychiatry. 2010; 81(1):31-6

Owens GP, Bennett JL, Lassmann H, O'Connor KC, Ritchie AM, Shearer A, et al.

Antibodies produced by clonally expanded plasma cells in multiple sclerosis cerebrospinal fluid Ann Neurol. 2009; 65(6):639-49

Owens GP, Ritchie AM, Burgoon MP, Williamson RA, Corboy JR, Gilden DH

Single-cell repertoire analysis demonstrates that clonal expansion is a prominent feature of the $B$ cell response in multiple sclerosis cerebrospinal fluid

J Immunol. 2003; 171(5):2725-33 
Parish CR

Fluorescent dyes for lymphocyte migration and proliferation studies

Immunol Cell Biol. 1999; 77(6):499-508

Park YW, Kee SJ, Cho YN, Lee EH, Lee HY, Kim EM, et al.

Impaired differentiation and cytotoxicity of natural killer cells in systemic lupus erythematosus

Arthritis Rheum. 2009; 60(6):1753-63

Patrikios P, Stadelmann C, Kutzelnigg A, Rauschka H, Schmidbauer M, Laursen H, et al.

Remyelination is extensive in a subset of multiple sclerosis patients

Brain. 2006; 129(Pt 12):3165-72

Pende D, Castriconi R, Romagnani P, Spaggiari GM, Marcenaro S, Dondero A, et al.

Expression of the DNAM-1 ligands, Nectin-2 (CD112) and poliovirus receptor (CD155), on dendritic cells: relevance for natural killer-dendritic cell interaction

Blood. 2006; 107(5):2030-6

Perini P, Wadhwa M, Buttarello M, Meager A, Facchinetti A, Thorpe R, et al.

Effect of IFNbeta and anti-IFNbeta antibodies on NK cells in multiple sclerosis patients

J Neuroimmunol. 2000; 105(1):91-5

Peterson JW, Bo L, Mork S, Chang A, Trapp BD

Transected neurites, apoptotic neurons, and reduced inflammation in cortical multiple sclerosis lesions Ann Neurol. 2001; 50(3):389-400

Philpott NJ, Turner AJ, Scopes J, Westby M, Marsh JC, Gordon-Smith EC, et al.

The use of 7-amino actinomycin $D$ in identifying apoptosis: simplicity of use and broad spectrum of application compared with other techniques

Blood. 1996; 87(6):2244-51

Piccioli D, Sbrana S, Melandri E, Valiante NM

Contact-dependent stimulation and inhibition of dendritic cells by natural killer cells

J Exp Med. 2002; 195(3):335-41

Pitt D, Nagelmeier IE, Wilson HC, Raine CS

Glutamate uptake by oligodendrocytes: Implications for excitotoxicity in multiple sclerosis Neurology. 2003; 61(8):1113-20

Planas R, Jelcic I, Schippling S, Martin R, Sospedra M

Natalizumab treatment perturbs memory- and marginal zone-like B-cell homing in secondary lymphoid organs in multiple sclerosis

Eur J Immunol. 2012; 42(3):790-8

Plantone D, Marti A, Frisullo G, lorio R, Damato V, Nociti V, et al.

Circulating CD56dim NK cells expressing perforin are increased in progressive multiple sclerosis

J Neuroimmunol. 2013; 265(1-2):124-7

Poirot L, Benoist C, Mathis D

Natural killer cells distinguish innocuous and destructive forms of pancreatic islet autoimmunity Proc Natl Acad Sci U S A. 2004; 101(21):8102-7

Polman C, Barkhof F, Sandberg-Wollheim M, Linde A, Nordle O, Nederman T, et al.

Treatment with laquinimod reduces development of active MRI lesions in relapsing MS

Neurology. 2005; 64(6):987-91

Polman CH, O'Connor PW, Havrdova E, Hutchinson M, Kappos L, Miller DH, et al.

A randomized, placebo-controlled trial of natalizumab for relapsing multiple sclerosis

N Engl J Med. 2006; 354(9):899-910 
Polman CH, Reingold SC, Banwell B, Clanet M, Cohen JA, Filippi M, et al.

Diagnostic criteria for multiple sclerosis: 2010 revisions to the McDonald criteria

Ann Neurol. 2011; 69(2):292-302

Polman CH, Reingold SC, Edan G, Filippi M, Hartung HP, Kappos L, et al.

Diagnostic criteria for multiple sclerosis: 2005 revisions to the "McDonald Criteria"

Ann Neurol. 2005; 58(6):840-6

Pridgeon C, Lennon GP, Pazmany L, Thompson RN, Christmas SE, Moots RJ

Natural killer cells in the synovial fluid of rheumatoid arthritis patients exhibit a CD56bright,CD94bright,CD158negative phenotype

Rheumatology (Oxford). 2003; 42(7):870-8

Putz EM, Gotthardt D, Hoermann G, Csiszar A, Wirth S, Berger A, et al.

CDK8-mediated STAT1-S727 phosphorylation restrains NK cell cytotoxicity and tumor surveillance

Cell Rep. 2013; 4(3):437-44

Putzki N, Baranwal MK, Tettenborn B, Limmroth V, Kreuzfelder E

Effects of natalizumab on circulating $B$ cells, $T$ regulatory cells and natural killer cells

Eur Neurol. 2010; 63(5):311-7

Qiu ZX, Zhang K, Qiu XS, Zhou M, Li WM

CD226 Gly307Ser association with multiple autoimmune diseases: a meta-analysis

Hum Immunol. 2013; 74(2):249-55

Rabinovich BA, Li J, Shannon J, Hurren R, Chalupny J, Cosman D, et al.

Activated, but not resting, $T$ cells can be recognized and killed by syngeneic NK cells

J Immunol. 2003; 170(7):3572-6

Ranger AM, Das MP, Kuchroo VK, Glimcher LH

B7-2 (CD86) is essential for the development of IL-4-producing T cells

Int Immunol. 1996; 8(10):1549-60

Rao SM, Leo GJ, Bernardin L, Unverzagt $F$

Cognitive dysfunction in multiple sclerosis. I. Frequency, patterns, and prediction

Neurology. 1991; 41(5):685-91

Raulet DH

Roles of the NKG2D immunoreceptor and its ligands

Nat Rev Immunol. 2003; 3(10):781-90

Rawlings JS, Rosler KM, Harrison DA

The JAK/STAT signaling pathway

J Cell Sci. 2004; 117(Pt 8):1281-3

Ridge SC, Sloboda AE, McReynolds RA, Levine S, Oronsky AL, Kerwar SS

Suppression of experimental allergic encephalomyelitis by mitoxantrone

Clin Immunol Immunopathol. 1985; 35(1):35-42

Rizzello V, Bonaccorsi I, Dongarra ML, Fink LN, Ferlazzo G

Role of natural killer and dendritic cell crosstalk in immunomodulation by commensal bacteria probiotics J Biomed Biotechnol. 2011; 2011:473097

Rodacki M, Svoren B, Butty V, Besse W, Laffel L, Benoist C, et al.

Altered natural killer cells in type 1 diabetic patients

Diabetes. 2007; 56(1):177-85 
Rudick RA, Stuart WH, Calabresi PA, Confavreux C, Galetta SL, Radue EW, et al. Natalizumab plus interferon beta-1a for relapsing multiple sclerosis N Engl J Med. 2006; 354(9):911-23

Ruffini F, Rossi S, Bergamaschi A, Brambilla E, Finardi A, Motta C, et al.

Laquinimod prevents inflammation-induced synaptic alterations occurring in experimental autoimmune encephalomyelitis

Mult Scler. 2013; 19(8):1084-94

Runström A, Leanderson T, Ohlsson L, Axelsson B

Inhibition of the development of chronic experimental autoimmune encephalomyelitis by laquinimod (ABR-215062) in IFN-beta k.o. and wild type mice J Neuroimmunol. 2006; 173(1-2):69-78

Saikali P, Antel JP, Newcombe J, Chen Z, Freedman M, Blain M, et al.

NKG2D-mediated cytotoxicity toward oligodendrocytes suggests a mechanism for tissue injury in multiple sclerosis

The Journal of neuroscience : the official journal of the Society for Neuroscience. $2007 ; 27(5): 1220-8$

Samson SI, Memet S, Vosshenrich CA, Colucci F, Richard O, Ndiaye D, et al.

Combined deficiency in IkappaBalpha and IkappaBepsilon reveals a critical window of NF-kappaB activity in natural killer cell differentiation

Blood. 2004; 103(12):4573-80

Sand KL, Knudsen E, Rolin J, Al-Falahi Y, Maghazachi AA

Modulation of natural killer cell cytotoxicity and cytokine release by the drug glatiramer acetate Cell Mol Life Sci. 2009; 66(8):1446-56

Saraste M, Irjala H, Airas L

Expansion of CD56Bright natural killer cells in the peripheral blood of multiple sclerosis patients treated with interferon-beta

Neurol Sci. 2007; 28(3):121-6

Satoh J, Kim SU, Kastrukoff LF

Absence of natural killer (NK) cell activity against oligodendrocytes in multiple sclerosis

J Neuroimmunol. 1990; 26(1):75-80

Sawcer S, Hellenthal G, Pirinen M, Spencer CC, Consortium IMSG, Consortium WTCC, et al. Genetic risk and a primary role for cell-mediated immune mechanisms in multiple sclerosis Nature. 2011; 476(7359):214-9

Scannevin RH, Chollate S, Jung MY, Shackett M, Patel H, Bista P, et al.

Fumarates promote cytoprotection of central nervous system cells against oxidative stress via the nuclear factor (erythroid-derived 2)-like 2 pathway

J Pharmacol Exp Ther. 2012; 341(1):274-84

Schirmer L, Antel JP, Brück W, Stadelmann C

Axonal loss and neurofilament phosphorylation changes accompany lesion development and clinical progression in multiple sclerosis

Brain Pathol. 2011; 21(4):428-40

Schulze-Topphoff U, Shetty A, Varrin-Doyer M, Molnarfi N, Sagan SA, Sobel RA, et al.

Laquinimod, a quinoline-3-carboxamide, induces type II myeloid cells that modulate central nervous system autoimmunity

PLoS One. 2012; 7(3):e33797 
Segal BM

The role of natural killer cells in curbing neuroinflammation

J Neuroimmunol. 2007; 191(1-2):2-7

Seidel UJ, Schlegel P, Lang P

Natural killer cell mediated antibody-dependent cellular cytotoxicity in tumor immunotherapy with therapeutic antibodies

Front Immunol. 2013; 4:76

Seth S, Maier MK, Qiu Q, Ravens I, Kremmer E, Forster R, et al.

The murine pan T cell marker CD96 is an adhesion receptor for CD155 and nectin-1

Biochem Biophys Res Commun. 2007; 364(4):959-65

Sharief MK, Thompson EJ

Intrathecal immunoglobulin M synthesis in multiple sclerosis. Relationship with clinical and cerebrospinal fluid parameters

Brain. 1991; 114 ( Pt 1A):181-95

Shi FD, Takeda K, Akira S, Sarvetnick N, Ljunggren HG

IL-18 directs autoreactive $\mathrm{T}$ cells and promotes autodestruction in the central nervous system via induction of IFN-gamma by NK cells

J Immunol. 2000; 165(6):3099-104

Shibuya K, Shirakawa J, Kameyama T, Honda S, Tahara-Hanaoka S, Miyamoto A, et al.

CD226 (DNAM-1) is involved in lymphocyte function-associated antigen 1 costimulatory signal for naive T cell differentiation and proliferation

J Exp Med. 2003; 198(12):1829-39

Simpson S, Jr., Taylor B, Blizzard L, Ponsonby AL, Pittas F, Tremlett H, et al.

Higher 25-hydroxyvitamin $D$ is associated with lower relapse risk in multiple sclerosis

Ann Neurol. 2010; 68(2):193-203

Singh S, Metz I, Amor S, van der Valk P, Stadelmann C, Brück W

Microglial nodules in early multiple sclerosis white matter are associated with degenerating axons

Acta Neuropathol. 2013; 125(4):595-608

Skarica M, Eckstein C, Whartenby KA, Calabresi PA

Novel mechanisms of immune modulation of natalizumab in multiple sclerosis patients

J Neuroimmunol. 2011; 235(1-2):70-6

Skulina C, Schmidt S, Dornmair K, Babbe H, Roers A, Rajewsky K, et al.

Multiple sclerosis: brain-infiltrating CD8+ T cells persist as clonal expansions in the cerebrospinal fluid and blood

Proc Natl Acad Sci U S A. 2004; 101(8):2428-33

Smeltz RB, Wolf NA, Swanborg RH

Inhibition of autoimmune $T$ cell responses in the DA rat by bone marrow-derived NK cells in vitro: implications for autoimmunity

J Immunol. 1999; 163(3):1390-7

Smith KJ, Lassmann $\mathrm{H}$

The role of nitric oxide in multiple sclerosis

Lancet Neurol. 2002; 1(4):232-41

Soldan SS, Alvarez Retuerto Al, Sicotte NL, Voskuhl RR

Immune modulation in multiple sclerosis patients treated with the pregnancy hormone estriol

J Immunol. 2003; 171(11):6267-74 
Sospedra M, Martin R

Immunology of multiple sclerosis

Annu Rev Immunol. 2005; 23:683-747

Spaggiari GM, Carosio R, Pende D, Marcenaro S, Rivera P, Zocchi MR, et al.

NK cell-mediated lysis of autologous antigen-presenting cells is triggered by the engagement of the phosphatidylinositol 3-kinase upon ligation of the natural cytotoxicity receptors NKp30 and NKp46 Eur J Immunol. 2001; 31(6):1656-65

Srinivasan R, Sailasuta N, Hurd R, Nelson S, Pelletier D

Evidence of elevated glutamate in multiple sclerosis using magnetic resonance spectroscopy at $3 \mathrm{~T}$

Brain. 2005; 128(Pt 5):1016-25

Stanietsky N, Rovis TL, Glasner A, Seidel E, Tsukerman P, Yamin R, et al.

Mouse TIGIT inhibits NK-cell cytotoxicity upon interaction with PVR

European Journal of Immunology. 2013; 43(8):2138-50

Stanietsky N, Simic H, Arapovic J, Toporik A, Levy O, Novik A, et al.

The interaction of TIGIT with PVR and PVRL2 inhibits human NK cell cytotoxicity

Proc Natl Acad Sci U S A. 2009; 106(42):17858-63

Steinman L

A brief history of $T(H) 17$, the first major revision in the $T(H) 1 / T(H) 2$ hypothesis of $T$ cell-mediated tissue damage

Nat Med. 2007; 13(2):139-45

Stromnes IM, Cerretti LM, Liggitt D, Harris RA, Goverman JM

Differential regulation of central nervous system autoimmunity by $T(H) 1$ and $T(H) 17$ cells

Nat Med. 2008; 14(3):337-42

Stromnes IM, Goverman JM

Active induction of experimental allergic encephalomyelitis

Nat Protoc. 2006; 1(4):1810-9

Stromnes IM, Goverman JM

Passive induction of experimental allergic encephalomyelitis

Nat Protoc. 2006; 1(4):1952-60

Sun D, Whitaker JN, Huang Z, Liu D, Coleclough C, Wekerle H, et al.

Myelin antigen-specific CD8+ T cells are encephalitogenic and produce severe disease in C57BL/6 mice

J Immunol. 2001; 166(12):7579-87

Tahara-Hanaoka S, Shibuya K, Onoda Y, Zhang H, Yamazaki S, Miyamoto A, et al.

Functional characterization of DNAM-1 (CD226) interaction with its ligands PVR (CD155) and nectin-2 (PRR-2/CD112)

Int Immunol. 2004; 16(4):533-8

Takahashi K, Miyake S, Kondo T, Terao K, Hatakenaka M, Hashimoto S, et al.

Natural killer type $\mathbf{2}$ bias in remission of multiple sclerosis

J Clin Invest. 2001; 107(5):R23-9

Takao S, Ishikawa T, Yamashita K, Uchiyama T

The rapid induction of HLA-E is essential for the survival of antigen-activated naive CD4 T cells from attack by NK cells

J Immunol. 2010; 185(10):6031-40 
Takeda K, Dennert G

The development of autoimmunity in C57BL/6 lpr mice correlates with the disappearance of natural killer type 1-positive cells: evidence for their suppressive action on bone marrow stem cell proliferation, B cell immunoglobulin secretion, and autoimmune symptoms

J Exp Med. 1993; 177(1):155-64

Takeda K, Oshima H, Hayakawa Y, Akiba H, Atsuta M, Kobata T, et al.

CD27-mediated activation of murine NK cells

J Immunol. 2000; 164(4):1741-5

Teitelbaum D, Fridkis-Hareli M, Arnon R, Sela M

Copolymer 1 inhibits chronic relapsing experimental allergic encephalomyelitis induced by proteolipid protein (PLP) peptides in mice and interferes with PLP-specific T cell responses

J Neuroimmunol. 1996; 64(2):209-17

Teitelbaum D, Meshorer A, Hirshfeld T, Arnon R, Sela M

Suppression of experimental allergic encephalomyelitis by a synthetic polypeptide

Eur J Immunol. 1971; 1(4):242-8

Teitelbaum D, Webb C, Bree M, Meshorer A, Arnon R, Sela M

Suppression of experimental allergic encephalomyelitis in Rhesus monkeys by a synthetic basic copolymer

Clin Immunol Immunopathol. 1974; 3(2):256-62

Teitelbaum D, Webb C, Meshorer A, Arnon R, Sela M

Protection against experimental allergic encephalomyelitis

Nature. 1972; 240(5383):564-6

Teitelbaum D, Webb C, Meshorer A, Arnon R, Sela M

Suppression by several synthetic polypeptides of experimental allergic encephalomyelitis induced in guinea pigs and rabbits with bovine and human basic encephalitogen

Eur J Immunol. 1973; 3(5):273-9

Thöne J, Ellrichmann G, Seubert S, Peruga I, Lee DH, Conrad R, et al.

Modulation of autoimmune demyelination by laquinimod via induction of brain-derived neurotrophic factor

Am J Pathol. 2012; 180(1):267-74

Trapp BD, Peterson J, Ransohoff RM, Rudick R, Mork S, Bo L

Axonal transection in the lesions of multiple sclerosis

N Engl J Med. 1998; 338(5):278-85

Trivedi PP, Roberts PC, Wolf NA, Swanborg RH

NK cells inhibit $T$ cell proliferation via p21-mediated cell cycle arrest

J Immunol. 2005; 174(8):4590-7

Trojano M, Pellegrini F, Fuiani A, Paolicelli D, Zipoli V, Zimatore GB, et al.

New natural history of interferon-beta-treated relapsing multiple sclerosis

Ann Neurol. 2007; 61(4):300-6

Tuohy VK, Sobel RA, Lu Z, Laursen RA, Lees MB

Myelin proteolipid protein: minimum sequence requirements for active induction of autoimmune encephalomyelitis in SWR/J and SJL/J mice

J Neuroimmunol. 1992; 39(1-2):67-74 
Vahlne G, Becker S, Brodin P, Johansson MH

IFN-gamma production and degranulation are differentially regulated in response to stimulation in murine natural killer cells

Scand J Immunol. 2008; 67(1):1-11

Vaknin-Dembinsky A, Balashov K, Weiner HL

IL-23 is increased in dendritic cells in multiple sclerosis and down-regulation of IL-23 by antisense oligos increases dendritic cell IL-10 production

J Immunol. 2006; 176(12):7768-74

van der Valk P, De Groot CJ

Staging of multiple sclerosis (MS) lesions: pathology of the time frame of MS

Neuropathol Appl Neurobiol. 2000; 26(1):2-10

Vandenbark AA, Huan J, Agotsch M, La Tocha D, Goelz S, Offner H, et al.

Interferon-beta-1a treatment increases CD56bright natural killer cells and CD4+CD25+ Foxp3 expression in subjects with multiple sclerosis

J Neuroimmunol. 2009; 215(1-2):125-8

Vaz F, Silva MR, Ascensao JL

Enhanced lymphokine-activated killer cell activity by an immunomodulator, Roquinimex

Br J Cancer. 1995; 72(6):1498-503

Vermes I, Haanen C, Steffens-Nakken H, Reutelingsperger C

A novel assay for apoptosis. Flow cytometric detection of phosphatidylserine expression on early apoptotic cells using fluorescein labelled Annexin V

J Immunol Methods. 1995; 184(1):39-51

Vervliet G, Claeys H, Van Haver H, Carton H, Vermylen C, Meulepas E, et al.

Interferon production and natural killer (NK) activity in leukocyte cultures from multiple sclerosis patients J Neurol Sci. 1983; 60(1):137-50

Viglietta V, Baecher-Allan C, Weiner HL, Hafler DA

Loss of functional suppression by CD4+CD25+ regulatory $\mathrm{T}$ cells in patients with multiple sclerosis

J Exp Med. 2004; 199(7):971-9

Vitale M, Della Chiesa M, Carlomagno S, Pende D, Arico M, Moretta L, et al.

NK-dependent DC maturation is mediated by TNFalpha and IFNgamma released upon engagement of the NKp30 triggering receptor

Blood. 2005; 106(2):566-71

Vivier E, Raulet DH, Moretta A, Caligiuri MA, Zitvogel L, Lanier LL, et al. Innate or adaptive immunity? The example of natural killer cells

Science. 2011; 331(6013):44-9

Vivier E, Tomasello E, Baratin M, Walzer T, Ugolini S

Functions of natural killer cells

Nat Immunol. 2008; 9(5):503-10

Vogel DY, Vereyken EJ, Glim JE, Heijnen PD, Moeton M, van der Valk P, et al.

Macrophages in inflammatory multiple sclerosis lesions have an intermediate activation status

J Neuroinflammation. 2013; 10:35

Vollmer TL, Sorensen PS, Selmaj K, Zipp F, Havrdova E, Cohen JA, et al.

A randomized placebo-controlled phase III trial of oral laquinimod for multiple sclerosis

J Neurol. 2014:1-11 
von Budingen HC, Kuo TC, Sirota M, van Belle CJ, Apeltsin L, Glanville J, et al.

$B$ cell exchange across the blood-brain barrier in multiple sclerosis

J Clin Invest. 2012; 122(12):4533-43

Vranes Z, Poljakovic Z, Marusic M

Natural killer cell number and activity in multiple sclerosis

J Neurol Sci. 1989; 94(1-3):115-23

Wallin MT, Culpepper WJ, Coffman P, Pulaski S, Maloni H, Mahan CM, et al.

The Gulf War era multiple sclerosis cohort: age and incidence rates by race, sex and service

Brain. 2012; 135(Pt 6):1778-85

Walsh MJ, Tourtellotte WW

Temporal invariance and clonal uniformity of brain and cerebrospinal $\operatorname{lgG}$, IgA, and IgM in multiple sclerosis

J Exp Med. 1986; 163(1):41-53

Wang PL, O'Farrell S, Clayberger C, Krensky AM

Identification and molecular cloning of tactile. A novel human T cell activation antigen that is a member of the Ig gene superfamily

J Immunol. 1992; 148(8):2600-8

Warnke C, Meyer zu Horste G, Hartung HP, Stuve O, Kieseier BC

Review of teriflunomide and its potential in the treatment of multiple sclerosis

Neuropsychiatr Dis Treat. 2009; 5:333-40

Watson CM, Davison AN, Baker D, O'Neill JK, Turk JL

Suppression of demyelination by mitoxantrone

Int J Immunopharmacol. 1991; 13(7):923-30

Wegner C, Esiri MM, Chance SA, Palace J, Matthews PM

Neocortical neuronal, synaptic, and glial loss in multiple sclerosis

Neurology. 2006; 67(6):960-7

Wegner C, Stadelmann C, Pförtner R, Raymond E, Feigelson S, Alon R, et al.

Laquinimod interferes with migratory capacity of $T$ cells and reduces IL-17 levels, inflammatory demyelination and acute axonal damage in mice with experimental autoimmune encephalomyelitis J Neuroimmunol. 2010; 227(1-2):133-43

Werfel T, Boeker M, Kapp A

Rapid expression of the CD69 antigen on T cells and natural killer cells upon antigenic stimulation of peripheral blood mononuclear cell suspensions

Allergy. 1997; 52(4):465-9

Wieczorek S, Hoffjan S, Chan A, Rey L, Harper L, Fricke H, et al.

Novel association of the CD226 (DNAM-1) Gly307Ser polymorphism in Wegener's granulomatosis and confirmation for multiple sclerosis in German patients

Genes Immun. 2009; 10(6):591-5

Willcox A, Richardson SJ, Bone AJ, Foulis AK, Morgan NG

Analysis of islet inflammation in human type 1 diabetes

Clinical and Experimental Immunology. 2008; 155:173 - 81

Willer CJ, Dyment DA, Risch NJ, Sadovnick AD, Ebers GC

Twin concordance and sibling recurrence rates in multiple sclerosis

Proc Natl Acad Sci U S A. 2003; 100(22):12877-82 
Wilson JL, Heffler LC, Charo J, Scheynius A, Bejarano MT, Ljunggren HG

Targeting of human dendritic cells by autologous NK cells

J Immunol. 1999; 163(12):6365-70

Winkler-Pickett R, Young HA, Cherry JM, Diehl J, Wine J, Back T, et al.

In vivo regulation of experimental autoimmune encephalomyelitis by NK cells: alteration of primary adaptive responses

J Immunol. 2008; 180(7):4495-506

Wolf NA, Swanborg RH

DA rat $\mathrm{NK}(+) \mathrm{CD} 3(-)$ cells inhibit autoreactive T-cell responses

J Neuroimmunol. 2001; 119(1):81-7

Wynn D, Kaufman M, Montalban X, Vollmer T, Simon J, Elkins J, et al.

Daclizumab in active relapsing multiple sclerosis (CHOICE study): a phase 2, randomised, double-blind, placebo-controlled, add-on trial with interferon beta

Lancet Neurol. 2010; 9(4):381-90

Xu W, Fazekas G, Hara H, Tabira T

Mechanism of natural killer (NK) cell regulatory role in experimental autoimmune encephalomyelitis

J Neuroimmunol. 2005; 163(1-2):24-30

Yabuhara A, Yang FC, Nakazawa T, Iwasaki Y, Mori T, Koike K, et al.

A killing defect of natural killer cells as an underlying immunologic abnormality in childhood systemic lupus erythematosus

J Rheumatol. 1996; 23(1):171-7

Yang JS, Xu LY, Xiao BG, Hedlund G, Link H

Laquinimod (ABR-215062) suppresses the development of experimental autoimmune encephalomyelitis, modulates the Th1/Th2 balance and induces the Th3 cytokine TGF-beta in Lewis rats

J Neuroimmunol. 2004; 156(1-2):3-9

Yednock TA, Cannon C, Fritz LC, Sanchez-Madrid F, Steinman L, Karin N

Prevention of experimental autoimmune encephalomyelitis by antibodies against alpha 4 beta 1 integrin

Nature. 1992; 356(6364):63-6

Yong VW

Differential mechanisms of action of interferon-beta and glatiramer aetate in MS

Neurology. 2002; 59(6):802-8

Yu X, Harden K, Gonzalez LC, Francesco M, Chiang E, Irving B, et al.

The surface protein TIGIT suppresses $T$ cell activation by promoting the generation of mature immunoregulatory dendritic cells

Nat Immunol. 2009; 10(1):48-57

Zamai L, Ponti C, Mirandola P, Gobbi G, Papa S, Galeotti L, et al.

NK cells and cancer

J Immunol. 2007; 178(7):4011-6

Zamvil SS, Mitchell DJ, Moore AC, Kitamura K, Steinman L, Rothbard JB

T-cell epitope of the autoantigen myelin basic protein that induces encephalomyelitis

Nature. 1986; 324(6094):258-60

Zhang AL, Colmenero P, Purath U, Teixeira de Matos C, Hueber W, Klareskog L, et al. Natural killer cells trigger differentiation of monocytes into dendritic cells Blood. 2007; 110(7):2484-93 
Zhang B, Yamamura T, Kondo T, Fujiwara M, Tabira T

Regulation of experimental autoimmune encephalomyelitis by natural killer (NK) cells

J Exp Med. 1997; 186(10):1677-87

Zhang G, Gurtu V, Kain SR, Yan G

Early detection of apoptosis using a fluorescent conjugate of annexin V

Biotechniques. 1997; 23(3):525-31

Zhang X, Koldzic DN, Izikson L, Reddy J, Nazareno RF, Sakaguchi S, et al.

IL-10 is involved in the suppression of experimental autoimmune encephalomyelitis by CD25+CD4+ regulatory $\mathrm{T}$ cells

Int Immunol. 2004; 16(2):249-56

Zhou J, Zhang J, Lichtenheld MG, Meadows GG

A role for NF-kappa B activation in perforin expression of NK cells upon IL-2 receptor signaling J Immunol. 2002; 169(3):1319-25

Ziemssen T, Kumpfel T, Klinkert WE, Neuhaus O, Hohlfeld R

Glatiramer acetate-specific T-helper 1- and 2-type cell lines produce BDNF: implications for multiple sclerosis therapy. Brain-derived neurotrophic factor

Brain. 2002; 125(Pt 11):2381-91 


\section{Curriculum vitae}

\section{Personal Data}

Name

Date of birth

Place of birth

Citizenship

Address

\section{Education}

Since $03 / 2011$

$10 / 2005-06 / 2010$

09/1996-06/2005
Martina Ott (née Hauck)

07.11 .1985

Bad Windsheim

German

Eichenstr. 36, 96482 Ahorn

Georg-August-University Goettingen, Germany

$\mathrm{PhD}$ student in the international program Molecular Medicine

Friedrich-Alexander-University Erlangen-Nuremberg, Germany

Diploma studies in Molecular Medicine (Grade 1.0)

Gymnasium Scheinfeld, Germany

Abitur (corresponds to A levels, Grade 1.0)

\section{Professional experience}

Since $10 / 2010$

University Medical Center Goettingen, Germany

Department of Neuropathology

PhD thesis: "Analyzing the effects of laquinimod on innate and adaptive immunity in mice with experimental autoimmune encephalomyelitis"

$11 / 2009-06 / 2010$

Friedrich-Alexander-University Erlangen-Nuremberg, Germany Department of Pharmacology Diploma thesis: "Analysis of interaction partners of $\mathrm{HCN}$ pacemaker channels using a modified yeast-two hybrid screen" 
$04 / 2009-07 / 2010$

$01 / 2009-4 / 2009$

$10 / 2008-12 / 2008$
Friedrich-Alexander-University Erlangen-Nuremberg, Germany

Department of Pharmacology

Group leader in the tutorial on Pharmacology for medical students

Max Planck Institute for Neurological Research, Cologne, Germany

Research internship

Rheinische Friedrich-Wilhelms University Bonn, Germany

Department of Molecular Psychiatry

Research internship

\section{Publications}

\section{Original Article}

2014

Ribes S, Meister T, Ott M, Redlich S, Janova H, Hanisch UK, Nessler S, Nau R

Intraperitoneal prophylaxis with $\mathrm{CpG}$ oligodeoxynucleotides protects neutropenic mice against intracerebral Escherichia coli K1 infection

J Neuroinflammation 2014, 11:14

\section{Poster presentations}

2012

Ott M, Wegner C, Nessler S, Brück W

Preventive laquinimod treatment shifts pro-inflammatory to regulatory $\mathbf{T}$ cells and reduces myeloid dendritic cells in experimental autoimmune encephalomyelitis

$28^{\text {th }}$ Congress of the European Committee for Treatment and Research in Multiple Sclerosis, October $10-13^{\text {th }} 2012$, Lyon, France 